\title{
Rehabilitation for distal radial fractures in adults (Review)
}

\author{
Handoll HHG, Elliott J
}

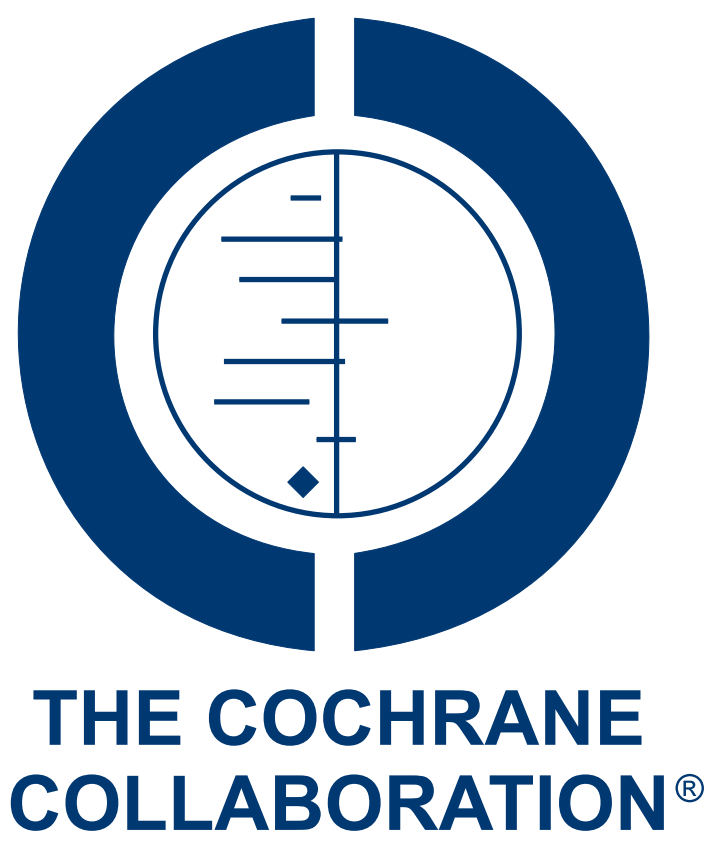

This is a reprint of a Cochrane review, prepared and maintained by The Cochrane Collaboration and published in The Cochrane Library 2015, Issue 9

http://www.thecochranelibrary.com

\section{WILEY}

Rehabilitation for distal radial fractures in adults (Review)

Copyright $\odot 2015$ The Cochrane Collaboration. Published by John Wiley \& Sons, Ltd. 
TABLE OF CONTENTS

HEADER . . . . . . . . . . . . . . . . . . . . . . . . . . . . . . . . . . . . . . . . . . . . . . . . .

ABSTRACT . . . . . . . . . . . . . . . . . . . . . . . . . . . . . . . . . . . . . . . . . . . . . . .

PLAIN LANGUAGE SUMMARY . . . . . . . . . . . . . . . . . . . . . . . . . . . . . . . . . . . . . 2

BACKGROUND . . . . . . . . . . . . . . . . . . . . . . . . . . . . . . . . . . . . . . . . 4

OBJECTIVES . . . . . . . . . . . . . . . . . . . . . . . . . . . . . . . . . . . . . . . . 5

METHODS . . . . . . . . . . . . . . . . . . . . . . . . . . . . . . . . . . . . . . .

RESULTS . . . . . . . . . . . . . . . . . . . . . . . . . . . . . . . . . . . . . . . . .

Figure 1. . . . . . . . . . . . . . . . . . . . . . . . . . . . . . . . . . . . . .

Figure 2. . . . . . . . . . . . . . . . . . . . . . . . . . . . . . . . . . . . . . 15

Figure 3. . . . . . . . . . . . . . . . . . . . . . . . . . . . . . 16

DISCUSSION . . . . . . . . . . . . . . . . . . . . . . . . . . . . . . . . . . . . . . 24

AUTHORS' CONCLUSIONS . . . . . . . . . . . . . . . . . . . . . . . . . . . . . . . . . . . . . . . .

ACKNOWLEDGEMENTS . . . . . . . . . . . . . . . . . . . . . . . . . . . . . . . . . . . . . . .

REFERENCES . . . . . . . . . . . . . . . . . . . . . . . . . . . . . . . . . . . . . . . . . . . .

CHARACTERISTICS OF STUDIES . . . . . . . . . . . . . . . . . . . . . . . . . . . . . . . . . . . . . . . . .

DATA AND ANALYSES . . . . . . . . . . . . . . . . . . . . . . . . . . . . . . . . . . . . . . . . . 108

Analysis 1.1. Comparison 1 Early (during immobilisation) occupational or hand therapy versus no intervention (control),

Outcome 1 Meeting criteria for attendance of post-immobilisation hand therapy group. . . . . . . . . . . 121

Analysis 1.2. Comparison 1 Early (during immobilisation) occupational or hand therapy versus no intervention (control),

Outcome 2 Grip strength (kg) at 4 weeks (post-immobilisation). . . . . . . . . . . . . . . . . . . .

Analysis 1.3. Comparison 1 Early (during immobilisation) occupational or hand therapy versus no intervention (control),

Outcome 3 Range of motion at 4 weeks (post-immobilisation). . . . . . . . . . . . . . . . . . . 122

Analysis 1.4. Comparison 1 Early (during immobilisation) occupational or hand therapy versus no intervention (control), Outcome 4 Oedema $(\mathrm{mL})$ at 4 weeks (post-immobilisation). $\quad . \quad$. . . . . . . . . . . . . . . . . . . . . . . 123

Analysis 1.5. Comparison 1 Early (during immobilisation) occupational or hand therapy versus no intervention (control), Outcome 5 Any pain at rest at 4 weeks (post-immobilisation). . . . . . . . . . . . . . . . . . . . . . .

Analysis 1.6. Comparison 1 Early (during immobilisation) occupational or hand therapy versus no intervention (control), Outcome 6 Finger mobility at 4 weeks (post-immobilisation). . . . . . . . . . . . . . . . . . . 124

Analysis 1.7. Comparison 1 Early (during immobilisation) occupational or hand therapy versus no intervention (control), Outcome 7 Complications.

Analysis 2.1. Comparison 2 Cyclic pneumatic soft tissue compression during immobilisation versus no intervention (control), Outcome 1 Grip strength (kg).

Analysis 2.2. Comparison 2 Cyclic pneumatic soft tissue compression during immobilisation versus no intervention (control), Outcome 2 Pinch strength $(\mathrm{kg})$.

Analysis 2.3. Comparison 2 Cyclic pneumatic soft tissue compression during immobilisation versus no intervention (control), Outcome 3 Range of motion (degrees).

Analysis 3.1. Comparison 3 Early (during external fixation) digit mobilisation programme versus no intervention (control), Outcome 1 Manual Ability Measure-36 - Taiwan version 45 questions (36 to 180: best result). . . . . . . . . . . 128

Analysis 3.2. Comparison 3 Early (during external fixation) digit mobilisation programme versus no intervention (control),

Outcome 2 Grip, pinch and 'three jaw chuck' pinch strengths (\% of uninvolved hand). . . . . . . . . . . . .
Analysis 3.3. Comparison 3 Early (during external fixation) digit mobilisation programme versus no intervention (control), Outcome 3 Range of motion (\% of other hand). . . . . . . . . . . . . . . . . . . . . . . . . . . . . .

Analysis 4.1. Comparison 4 Pulsed electromagnetic field (PEMF) (during cast immobilisation) versus no intervention (control), Outcome 1 PRWE scores at 2 to 3 days after cast removal.

Analysis 4.2. Comparison 4 Pulsed electromagnetic field (PEMF) (during cast immobilisation) versus no intervention (control), Outcome 2 Range of motion at day 2 to 3 after cast removal.

Analysis 4.3. Comparison 4 Pulsed electromagnetic field (PEMF) (during cast immobilisation) versus no intervention (control), Outcome 3 Hand oedema: difference between hands in circumference ( $\mathrm{mm})$.

Analysis 4.4. Comparison 4 Pulsed electromagnetic field (PEMF) (during cast immobilisation) versus no intervention (control), Outcome 4 Complications. 
Analysis 5.1. Comparison 5 Cross-education (strengthening exercises for opposite hand) versus no intervention (control), Outcome 1 Patient-Rated Wrist Evaluation (PRWE) (0 to 150: worst results). . . . . . . . . . . . . .

Analysis 5.2. Comparison 5 Cross-education (strengthening exercises for opposite hand) versus no intervention (control), Outcome 2 Grip strength of fractured hand $(\mathrm{kg})$.

Analysis 5.3. Comparison 5 Cross-education (strengthening exercises for opposite hand) versus no intervention (control), Outcome 3 Range of motion: supination/pronation (degrees). . . . . . . . . . . . . . . . . . . . . . . . Analysis 5.4. Comparison 5 Cross-education (strengthening exercises for opposite hand) versus no intervention (control), Outcome 4 Range of motion: flexion/extension (degrees). $\quad . \quad$. . . . . . . . . . . . . . . . . . . . . . . . .

Analysis 6.1. Comparison 6 Physiotherapy (one session for home exercises) versus no intervention (control) (postimmobilisation), Outcome 1 PRWE scores at 3 and 6 weeks. . . . . . . . . . . . . . . . . . . . . . .

Analysis 6.2. Comparison 6 Physiotherapy (one session for home exercises) versus no intervention (control) (postimmobilisation), Outcome 2 QuickDASH scores at 3 and 6 weeks. . . . . . . . . . . . . . . . .

Analysis 6.3. Comparison 6 Physiotherapy (one session for home exercises) versus no intervention (control) (postimmobilisation), Outcome 3 Grip strength (kg) at 3 and 6 weeks. . . . . . . . . . . . . . . . . . . . .

Analysis 6.4. Comparison 6 Physiotherapy (one session for home exercises) versus no intervention (control) (postimmobilisation), Outcome 4 Range of motion at 6 weeks (degrees). . . . . . . . . . . . . . . . . . . .

Analysis 6.5. Comparison 6 Physiotherapy (one session for home exercises) versus no intervention (control) (postimmobilisation), Outcome 5 Change in range of motion at 6 weeks (degrees). . . . . . . . . . . . . . .

Analysis 6.6. Comparison 6 Physiotherapy (one session for home exercises) versus no intervention (control) (postimmobilisation), Outcome 6 Complications. . . . . . . . . . . . . . . . . . . . . . . . . .

Analysis 6.7. Comparison 6 Physiotherapy (one session for home exercises) versus no intervention (control) (postimmobilisation), Outcome 7 Request for more physiotherapy. . . . . . . . . . . . . . . . . .

Analysis 7.1. Comparison 7 Post-immobilisation occupational or physiotherapy versus no intervention (control), Outcome 1 Patient-Rated Wrist Evaluation (PRWE) at 24 weeks (\%: $100 \%$ = worst results). . . . . . . . . . . 143

Analysis 7.2. Comparison 7 Post-immobilisation occupational or physiotherapy versus no intervention (control), Outcome 2 Activities of daily living scores (\% of unaffected side). . . . . . . . . . . . . . . . . . . . . . . . . 144

Analysis 7.3. Comparison 7 Post-immobilisation occupational or physiotherapy versus no intervention (control), Outcome 3 Grip strength $(\mathrm{kg}) . \quad$. . . . . . . . . . . . . . . . . . . . . . . . . . . . . . . . . . . . . . . . . 144

Analysis 7.4. Comparison 7 Post-immobilisation occupational or physiotherapy versus no intervention (control), Outcome 4 Grip strength (\% of unaffected side). . . . . . . . . . . . . . . . . . . . . . . . . . . . . . . . . .

Analysis 7.5. Comparison 7 Post-immobilisation occupational or physiotherapy versus no intervention (control), Outcome 5 Pain (VAS: none to worst imaginable at $10 \mathrm{~cm}$ ). $\quad . \quad$. . . . . . . . . . . . . . . . . . . . . . . . . . 146

Analysis 7.6. Comparison 7 Post-immobilisation occupational or physiotherapy versus no intervention (control), Outcome 6 Range of motion at 24 weeks.

Analysis 7.7. Comparison 7 Post-immobilisation occupational or physiotherapy versus no intervention (control), Outcome 7 Range of motion (\% of unaffected side) at 3 months. . . . . . . . . . . . . . . . . . . . . . . . . . 147

Analysis 7.8. Comparison 7 Post-immobilisation occupational or physiotherapy versus no intervention (control), Outcome 8 Range of motion (\% of unaffected side) at 6 months. . . . . . . . . . . . . . . . . . . . . . . . . . . . $\quad 148$

Analysis 7.9. Comparison 7 Post-immobilisation occupational or physiotherapy versus no intervention (control), Outcome 9 Number of treatments. . . . . . . . . . . . . . . . . . . . . . . . . . . . . . . 148

Analysis 7.10. Comparison 7 Post-immobilisation occupational or physiotherapy versus no intervention (control), Outcome 10 Complications. . . . . . . . . . . . . . . . . . . . . . . . . . . . . . . . . 149

Analysis 8.1. Comparison 8 Continuous passive motion (CPM) (post-external fixation) versus no intervention (control), Outcome 1 Time to recover independence (weeks). . . . . . . . . . . . . . . . . . . . . . . . . . . . .

Analysis 9.1. Comparison 9 Pulsed electromagnetic field (PEMF) (post-immobilisation) versus sham control, Outcome 1 Pain and volume at day 5.

Analysis 9.2. Comparison 9 Pulsed electromagnetic field (PEMF) (post-immobilisation) versus sham control, Outcome 2 Range of motion at day 5.

Analysis 10.1. Comparison 10 Ice (post-immobilisation) versus no ice (control), Outcome 1 Pain and volume at day 5. Analysis 10.2. Comparison 10 Ice (post-immobilisation) versus no ice (control), Outcome 2 Range of motion at day 5.

Analysis 11.1. Comparison 11 Pulsed electromagnetic field (PEMF) plus ice (post-immobilisation) versus no intervention (control), Outcome 1 Pain and volume at day 5. . . . . . . . . . . . . . . . . . . . . . . . . . . . .

Rehabilitation for distal radial fractures in adults (Review)

Copyright () 2015 The Cochrane Collaboration. Published by John Wiley \& Sons, Ltd. 
Analysis 11.2. Comparison 11 Pulsed electromagnetic field (PEMF) plus ice (post-immobilisation) versus no intervention (control), Outcome 2 Range of motion at day 5.

Analysis 12.1. Comparison 12 Passive mobilisation (post-immobilisation) versus no intervention (control), Outcome 1 Grip strength $(\mathrm{kg})$ at 6 weeks.

Analysis 12.2. Comparison 12 Passive mobilisation (post-immobilisation) versus no intervention (control), Outcome 2 Range of motion at 6 weeks. . . . . . . . . . . . . . . . . . . . . . . . . . . . . . . . . . . . . . . . . . . .

Analysis 12.3. Comparison 12 Passive mobilisation (post-immobilisation) versus no intervention (control), Outcome 3

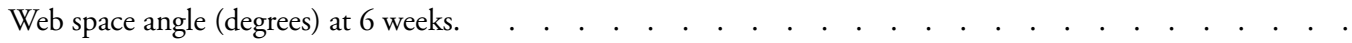

Analysis 12.4. Comparison 12 Passive mobilisation (post-immobilisation) versus no intervention (control), Outcome 4 Wrist extension at discharge $(4$ weeks).

Analysis 12.5. Comparison 12 Passive mobilisation (post-immobilisation) versus no intervention (control), Outcome 5 Number of treatments. . . . . . . . . . . . . . . . . . . . . . . . . . . . . . . . . . . . . . . . . . . .

Analysis 12.6. Comparison 12 Passive mobilisation (post-immobilisation) versus no intervention (control), Outcome 6

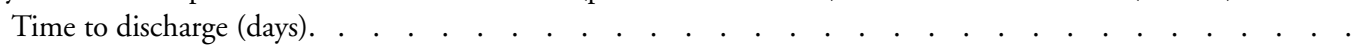

Analysis 12.7. Comparison 12 Passive mobilisation (post-immobilisation) versus no intervention (control), Outcome 7 Complications at 6 weeks.

Analysis 13.1. Comparison 13 Low frequency, long-wave ultrasound (post-immobilisation) versus sham intervention, Outcome 1 Greater than $30 \%$ loss of wrist motion (flexion-extension) at 8 weeks.

Analysis 13.2. Comparison 13 Low frequency, long-wave ultrasound (post-immobilisation) versus sham intervention, Outcome 2 Referral for physiotherapy. . . . . . . . . . . . . . . . . . . . . . . . . .

Analysis 14.1. Comparison 14 Whirlpool (post-immobilisation) versus towel (control), Outcome 1 Grip strength at end of treatment $(\mathrm{kg}) . \quad$. . . . . . . . . . . . . . . . . . . . . . . . . . . . . . . . . . . . . . . . . .

Analysis 14.2. Comparison 14 Whirlpool (post-immobilisation) versus towel (control), Outcome 2 Pain (scale 0: no pain to 5: excruciating) at end of treatment. . . . . . . . . . . . . . . . . . . . . . . . . . . . . . . . . . 161

Analysis 14.3. Comparison 14 Whirlpool (post-immobilisation) versus towel (control), Outcome 3 Range of motion at end of treatment. . . . . . . . . . . . . . . . . . . . . . . . . . . . . . . . . . . 162

Analysis 14.4. Comparison 14 Whirlpool (post-immobilisation) versus towel (control), Outcome 4 Finger flexion at end of treatment. . . . . . . . . . . . . . . . . . . . . . . . . . . . . . . . . . . . 163

Analysis 14.5. Comparison 14 Whirlpool (post-immobilisation) versus towel (control), Outcome 5 Oedema (ml). . $\quad 164$

Analysis 15.1. Comparison 15 Dynamic wrist extension splint versus no intervention (control) (post-immobilisation), Outcome 1 Patient-Rated Wrist Evaluation (PRWE) (\%: 100\% = worst results). . . . . . . . . . . .

Analysis 15.2. Comparison 15 Dynamic wrist extension splint versus no intervention (control) (post-immobilisation), Outcome 2 Canadian Occupational Performance Measure at 12 weeks. . . . . . . . . . . . . . . . . . .

Analysis 15.3. Comparison 15 Dynamic wrist extension splint versus no intervention (control) (post-immobilisation), Outcome 3 Range of motion at 12 weeks. . . . . . . . . . . . . . . . . . . . . . . . .

Analysis 16.1. Comparison 16 Post-immobilisation physiotherapy versus instructions from physician, Outcome 1 Wrist extension (degrees) at 6 weeks. . . . . . . . . . . . . . . . . . . . . . . . . . . . . . . . . . . . . . . ..

Analysis 17.1. Comparison 17 Pulsed electromagnetic field (PEMF) versus ice (post-immobilisation), Outcome 1 Pain and volume at day 5. . . . . . . . . . . . . . . . . . . . . . . . . . . . . . . . . . . . . . 167

Analysis 17.2. Comparison 17 Pulsed electromagnetic field (PEMF) versus ice (post-immobilisation), Outcome 2 Range of motion at day 5. . . . . . . . . . . . . . . . . . . . . . . . . . . . . . . . . . 168

Analysis 18.1. Comparison 18 'Manual Edema Mobilization' (MEM) versus 'traditional' oedema treatment, Outcome 1 Canadian Occupational Performance Measure at 9 weeks (clinically important improvement). . . . . . . .

Analysis 18.2. Comparison 18 'Manual Edema Mobilization' (MEM) versus 'traditional' oedema treatment, Outcome 2 Pain (VAS: 0 to 100: worst pain). . . . . . . . . . . . . . . . . . . . . . . . . . . .

Analysis 18.3. Comparison 18 'Manual Edema Mobilization' (MEM) versus 'traditional' oedema treatment, Outcome 3 Complications.

Analysis 18.4. Comparison 18 'Manual Edema Mobilization' (MEM) versus 'traditional' oedema treatment, Outcome 4 Number of occupational therapy sessions. . . . . . . . . . . . . . . . . . . . . . . . . . . . . .

Analysis 18.5. Comparison 18 'Manual Edema Mobilization' (MEM) versus 'traditional' oedema treatment, Outcome 5 Receiving oedema treatment after scheduled period. . . . . . . . . . . . . . . . . . . . . . . . . . .

Analysis 18.6. Comparison 18 'Manual Edema Mobilization' (MEM) versus 'traditional' oedema treatment, Outcome 6 Oedema: volume difference between injured and non-injured side $(\mathrm{mL})$.

Rehabilitation for distal radial fractures in adults (Review)

Copyright () 2015 The Cochrane Collaboration. Published by John Wiley \& Sons, Ltd. 
Analysis 19.1. Comparison 19 Supervised training by physiotherapist versus instructions by physician (from definitive treatment), Outcome 1 Strength and power at 12 weeks.

Analysis 19.2. Comparison 19 Supervised training by physiotherapist versus instructions by physician (from definitive treatment), Outcome 2 Range of motion at 12 weeks. . . . . . . . . . . . . . . . . . . . . . . . .

Analysis 20.1. Comparison 20 Physiotherapy/occupational therapy versus home exercise programme (post-surgery), Outcome 1 PRWE scores at 6 weeks (0: normal to 150: worst outcome).

Analysis 20.2. Comparison 20 Physiotherapy/occupational therapy versus home exercise programme (post-surgery), Outcome 2 DASH score (0 to 100: higher scores = worse upper-extremity function).

Analysis 20.3. Comparison 20 Physiotherapy/occupational therapy versus home exercise programme (post-surgery), Outcome 3 Mayo wrist score (0 to 100: higher scores = best functional outcome).

Analysis 20.4. Comparison 20 Physiotherapy/occupational therapy versus home exercise programme (post-surgery), Outcome 4 Pain at rest (VAS: 0 to 10: worst pain). . . . . . . . . . . . . . . . . . . . . . . . . . .

Analysis 20.5. Comparison 20 Physiotherapy/occupational therapy versus home exercise programme (post-surgery), Outcome 5 Grip strength $(\mathrm{kg})$.

Analysis 20.6. Comparison 20 Physiotherapy/occupational therapy versus home exercise programme (post-surgery), Outcome 6 Pinch strength $(\mathrm{kg})$.

Analysis 20.7. Comparison 20 Physiotherapy/occupational therapy versus home exercise programme (post-surgery), Outcome 7 Range of motion at 3 months. . . . . . . . . . . . . . . . . . . . . . . . . . . . .

Analysis 20.8. Comparison 20 Physiotherapy/occupational therapy versus home exercise programme (post-surgery), Outcome 8 Range of motion at 6 months. . . . . . . . . . . . . . . . . . . . . . . . . . . . . .

Analysis 20.9. Comparison 20 Physiotherapy/occupational therapy versus home exercise programme (post-surgery), Outcome 9 Complications.

Analysis 21.1. Comparison 21 Accelerated (start 2 weeks) versus usual (start 6 weeks) rehabilitation post-surgery, Outcome 1 DASH score (0 to 100: higher scores = worse upper-extremity function). . . . . . . . . . . . . . . . 180

Analysis 21.2. Comparison 21 Accelerated (start 2 weeks) versus usual (start 6 weeks) rehabilitation post-surgery, Outcome 2 Grip strength (lb)

Analysis 21.3. Comparison 21 Accelerated (start 2 weeks) versus usual (start 6 weeks) rehabilitation post-surgery, Outcome

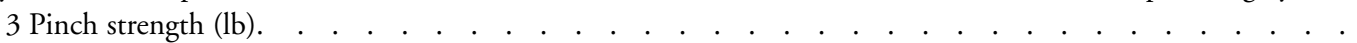

Analysis 21.4. Comparison 21 Accelerated (start 2 weeks) versus usual (start 6 weeks) rehabilitation post-surgery, Outcome

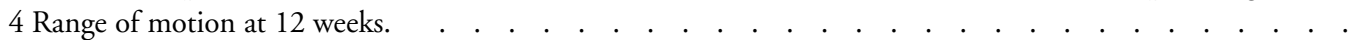

Analysis 21.5. Comparison 21 Accelerated (start 2 weeks) versus usual (start 6 weeks) rehabilitation post-surgery, Outcome

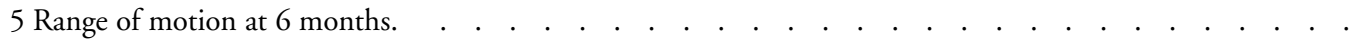

Analysis 21.6. Comparison 21 Accelerated (start 2 weeks) versus usual (start 6 weeks) rehabilitation post-surgery, Outcome 6 Complications. . . . . . . . . . . . . . . . . . . . . . . . . . . . . . . 184

ADDITIONAL TABLES . . . . . . . . . . . . . . . . . . . . . . . . . . . . . . 184

APPENDICES . . . . . . . . . . . . . . . . . . . . . . . . . . . . . . . . . 215

WHAT'S NEW . . . . . . . . . . . . . . . . . . . . . . . . . . . . . . . . . . . . . . 220

HISTORY . . . . . . . . . . . . . . . . . . . . . . . . . . . . . . . . . . . . . . . . . . . . 220

CONTRIBUTIONS OF AUTHORS . . . . . . . . . . . . . . . . . . . . . . . . . . . . . . . . . . . . 221

DECLARATIONS OF INTEREST . . . . . . . . . . . . . . . . . . . . . . . . . . . . . . . 221

SOURCES OF SUPPORT . . . . . . . . . . . . . . . . . . . . . . . . . . . . . . . . . . . 222

DIFFERENCES BETWEEN PROTOCOL AND REVIEW . . . . . . . . . . . . . . . . . . . . . . . . . . 222

INDEX TERMS . . . . . . . . . . . . . . . . . . . . . . . . . . . . . . . . . 222

Rehabilitation for distal radial fractures in adults (Review) 


\title{
[Intervention Review]
}

\section{Rehabilitation for distal radial fractures in adults}

\author{
Helen HG Handoll ${ }^{1}$, Joanne Elliott ${ }^{2}$ \\ ${ }^{1}$ Health and Social Care Institute, Teesside University, Middlesbrough, UK. ${ }^{2}$ Centre for Musculoskeletal Research, The University of \\ Manchester, Medical School, Manchester, UK \\ Contact address: Helen HG Handoll, Health and Social Care Institute, Teesside University, Middlesbrough, Tees Valley, TS1 3BA, \\ UK. h.handoll@tees.ac.uk.H.Handoll@ed.ac.uk.
}

Editorial group: Cochrane Bone, Joint and Muscle Trauma Group.

Publication status and date: New search for studies and content updated (conclusions changed), published in Issue 9, 2015.

Review content assessed as up-to-date: 12 January 2015.

Citation: Handoll HHG, Elliott J. Rehabilitation for distal radial fractures in adults. Cochrane Database of Systematic Reviews 2015, Issue 9. Art. No.: CD003324. DOI: 10.1002/14651858.CD003324.pub3.

Copyright (C) 2015 The Cochrane Collaboration. Published by John Wiley \& Sons, Ltd.

\begin{abstract}
A B S T R A C T
Background

Fracture of the distal radius is a common clinical problem, particularly in older people with osteoporosis. There is considerable variation in the management, including rehabilitation, of these fractures. This is an update of a Cochrane review first published in 2002 and last updated in 2006.
\end{abstract}

\section{Objectives}

To examine the effects of rehabilitation interventions in adults with conservatively or surgically treated distal radial fractures.

\section{Search methods}

We searched the Cochrane Bone, Joint and Muscle Trauma Group Specialised Register, the Cochrane Central Register of Controlled Trials (CENTRAL 2014; Issue 12), MEDLINE, EMBASE, CINAHL, AMED, PEDro, OTseeker and other databases, trial registers, conference proceedings and reference lists of articles. We did not apply any language restrictions. The date of the last search was 12 January 2015.

\section{Selection criteria}

Randomised controlled trials (RCTs) or quasi-RCTs evaluating rehabilitation as part of the management of fractures of the distal radius sustained by adults. Rehabilitation interventions such as active and passive mobilisation exercises, and training for activities of daily living, could be used on their own or in combination, and be applied in various ways by various clinicians.

\section{Data collection and analysis}

The review authors independently screened and selected trials, and reviewed eligible trials. We contacted study authors for additional information. We did not pool data.

\section{Main results}

We included 26 trials, involving 1269 mainly female and older patients. With few exceptions, these studies did not include people with serious fracture or treatment-related complications, or older people with comorbidities and poor overall function that would have precluded trial participation or required more intensive treatment. Only four of the 23 comparisons covered by these 26 trials were evaluated by more than one trial. Participants of 15 trials were initially treated conservatively, involving plaster cast immobilisation. Initial treatment was surgery (external fixation or internal fixation) for all participants in five trials. Initial treatment was either surgery

Rehabilitation for distal radial fractures in adults (Review)

Copyright $\odot 2015$ The Cochrane Collaboration. Published by John Wiley \& Sons, Ltd. 
or plaster cast alone in six trials. Rehabilitation started during immobilisation in seven trials and after post-immobilisation in the other 19 trials. As well as being small, the majority of the included trials had methodological shortcomings and were at high risk of bias, usually related to lack of blinding, that could affect the validity of their findings. Based on GRADE criteria for assessment quality, we rated the evidence for each of the 23 comparisons as either low or very low quality; both ratings indicate considerable uncertainty in the findings.

For interventions started during immobilisation, there was very low quality evidence of improved hand function for hand therapy compared with instructions only at four days after plaster cast removal, with some beneficial effects continuing one month later (one trial, 17 participants). There was very low quality evidence of improved hand function in the short-term, but not in the longer-term (three months), for early occupational therapy (one trial, 40 participants), and of a lack of differences in outcome between supervised and unsupervised exercises (one trial, 96 participants).

Four trials separately provided very low quality evidence of clinically marginal benefits of specific interventions applied in addition to standard care (therapist-applied programme of digit mobilisation during external fixation (22 participants); pulsed electromagnetic field (PEMF) during cast immobilisation (60 participants); cyclic pneumatic soft tissue compression using an inflatable cuff placed under the plaster cast (19 participants); and cross-education involving strength training of the non-fractured hand during cast immobilisation with or without surgical repair (39 participants)).

For interventions started post-immobilisation, there was very low quality evidence from one study (47 participants) of improved function for a single session of physiotherapy, primarily advice and instructions for a home exercise programme, compared with 'no intervention' after cast removal. There was low quality evidence from four heterogeneous trials (30, 33, 66 and 75 participants) of a lack of clinically important differences in outcome in patients receiving routine physiotherapy or occupational therapy in addition to instructions for home exercises versus instructions for home exercises from a therapist. There was very low quality evidence of better short-term hand function in participants given physiotherapy than in those given either instructions for home exercises by a surgeon (16 participants, one trial) or a progressive home exercise programme (20 participants, one trial). Both trials (46 and 76 participants) comparing physiotherapy or occupational therapy versus a progressive home exercise programme after volar plate fixation provided low quality evidence in favour of a structured programme of home exercises preceded by instructions or coaching. One trial (63 participants) provided very low quality evidence of a short-term, but not persisting, benefit of accelerated compared with usual rehabilitation after volar plate fixation.

For trials testing single interventions applied post-immobilisation, there was very low quality evidence of no clinically significant differences in outcome in patients receiving passive mobilisation (69 participants, two trials), ice (83 participants, one trial), PEMF (83 participants, one trial), PEMF plus ice (39 participants, one trial), whirlpool immersion (24 participants, one trial), and dynamic extension splint for patients with wrist contracture (40 participants, one trial), compared with no intervention. This finding applied also to the trial (44 participants) comparing PEMF versus ice, and the trial (29 participants) comparing manual oedema mobilisation versus traditional oedema treatment. There was very low quality evidence from single trials of a short-term benefit of continuous passive motion post-external fixation (seven participants), intermittent pneumatic compression (31 participants) and ultrasound (38 participants).

\section{Authors' conclusions}

The available evidence from RCTs is insufficient to establish the relative effectiveness of the various interventions used in the rehabilitation of adults with fractures of the distal radius. Further randomised trials are warranted. However, in order to optimise research effort and engender the large multicentre randomised trials that are required to inform practice, these should be preceded by research that aims to identify priority questions.

\section{PLAIN LANGUAGE SUMMARY}

\section{Rehabilitation as part of treatment for adults with a broken wrist}

\section{Background and aim}

Particularly in older women, a broken wrist (comprising a fracture at the lower end of the radius, one of the two forearm bones) can result from a fall onto an outstretched hand. Treatment usually includes putting the bone fragments back in place, if badly displaced, and immobilising the wrist in a plaster cast. Surgery may be considered for more seriously displaced fractures. Rehabilitation with

Rehabilitation for distal radial fractures in adults (Review)

Copyright @ 2015 The Cochrane Collaboration. Published by John Wiley \& Sons, Ltd. 
interventions such as exercises and other physical interventions is used to help prevent complications, such as stiffness and aching, restore function and speed up recovery.

This review set out to evaluate the effects, primarily on wrist function, of different rehabilitation interventions for treating these injuries.

\section{Search results}

We searched the scientific literature up to January 2015 and found 26 randomised controlled studies, involving 1269 mainly female and older patients. Only four of the 23 treatment comparisons covered by these 26 studies were tested by more than one study. Participants of 15 studies were initially treated with plaster cast immobilisation. Some or all participants in the other 11 studies were treated with surgery. In seven studies, the rehabilitation intervention being tested started during wrist immobilisation. In the other 19 studies, rehabilitation started when the cast had been removed.

All studies were small and were designed in a way that may affect the reliability of their findings. Most studies did not report on patientreported outcome measures of function and did not follow up patients for long enough. We judged the quality of the reported evidence as either low or very low and thus we are not confident that the results described below are true.

\section{Key results}

Interventions started during immobilisation

Two studies provided very low quality evidence that rehabilitation (hand therapy or task-orientated therapy) improved hand function after the plaster cast was removed, but not in the longer-term. One study provided very low quality evidence that outcome after supervised exercises did not differ from outcome after unsupervised exercises. Four studies provided very low quality evidence of some slight benefits of four different single methods of rehabilitation that were given with standard care.

Interventions started post-immobilisation, mainly after removal of the plaster cast

There was very low quality evidence from one study of improved function for a single session of physiotherapy, primarily advice and instructions for a home exercise programme, compared with 'no intervention' after cast removal. There was low quality evidence from four very different studies of no clinically important differences in outcome in patients receiving routine physiotherapy or occupational therapy in addition to instructions for home exercises versus instructions for home exercises from a therapist. There was very low quality evidence of better short-term hand function in the participants given physiotherapy than in those given either instructions for home exercises by a surgeon (one study) or a progressive home exercise programme (one study). Both studies comparing physiotherapy or occupational therapy versus a progressive home exercise programme after surgery involving plate fixation (a metal plate and screws are used to hold the broken bone in place) found low quality evidence in favour of a structured programme of home exercises preceded by instructions or coaching. One study provided very low quality evidence of a short-term, but not persisting, benefit of accelerated compared with usual rehabilitation after surgery involving plate fixation.

For studies testing single interventions applied post-immobilisation, there was very low quality evidence of no clinically significant differences in outcome in patients receiving passive mobilisation, ice, pulsed electromagnetic field (PEMF), PEMF plus ice, whirlpool immersion, and a dynamic extension splint for patients with a stiff wrist, compared with no intervention. This finding applied also to single studies comparing PEMF versus ice, and a new type of massage treatment for swelling when compared with the traditional approach. There was very low quality evidence from single studies of a short-term benefit of continuous passive motion immediately after removal of external fixation, intermittent pneumatic compression and ultrasound therapy.

\section{Conclusions}

We concluded that there was not enough evidence available to determine the best form of rehabilitation for people with wrist fractures. Priority questions need to be identified before further studies are done.

Rehabilitation for distal radial fractures in adults (Review) 


\section{B A C K G R O U N D}

\section{Description of the condition}

Fracture of the distal radius is one of the most common fractures in many predominantly white and older populations (Sahlin 1990; Singer 1998). It has been estimated that a 50-year-old white woman in the USA or Northern Europe has a 15\% lifetime risk of a distal radius fracture; whereas a white man of the same age has a lifetime risk of a little over $2 \%$ (Cummings 1985). A prospective survey, conducted in six centres in the UK, of Colles' fracture in patients aged 35 years and above, reported the overall annual incidence of this fracture to be $9 / 10,000$ in men and 37/10,000 in women (O'Neill 2001). In 2000, an incidence of 195 distal radius fractures per 100,000 adults was reported for the population $(534,715$ adults) served by the Edinburgh Royal Infirmary (Court-Brown 2006). Court-Brown 2006 reported that the average age of patients with these fractures was 56 years $(46 \%$ were over 65 years) and that the male:female ratio was 31:69. However, this hides the bimodal distribution of the age data, with a crossover of the two peaks of incidence at around 50 years of age; the second and higher peak being populated mainly by older women (see Figure 5 within Costa 2015). Distal radius fractures are usually treated on an outpatient basis, with around $20 \%$ of patients (mainly older people) requiring hospital admission (Cummings 1985; O'Neill 2001). This percentage is likely to be underestimated given the increasing use of surgery and the ageing population (Nellans 2012).

Most fractures of the distal radius in older people result from low-energy trauma, such as a fall from standing height or less. In younger adults, these injuries are usually sustained through highenergy trauma, such as a traffic accident. The pattern of incidence reflects the bone loss from osteoporosis in older people, as well as an increased number of falls by older women (Nguyen 2001).

These fractures are generally closed and usually involve displacement of fracture fragments. They may be either extra-articular (leaving the joint surface of the distal radius intact) or intra-articular (the joint surface is disrupted). Numerous classifications have been devised to define and group different fracture patterns (Chitnavis 1999). Simple classifications based on clinical appearance, and often named after those who described them, remain in common use. In particular, "Colles' fracture" is still the terminology used for a fracture in which there is an obvious and typical clinical deformity (commonly referred to as a 'dinner fork' deformity) of dorsal displacement, dorsal angulation, dorsal comminution (fragmentation), and radial shortening.

The majority of distal radial fractures are treated conservatively (non-operatively). This usually involves the reduction of the fracture if displaced, and forearm immobilisation in a plaster cast or brace for around six weeks. Surgical treatment usually involves either closed or open reduction, followed by external or internal fixation and a similar period of immobilisation. Since the last version of the review, there has been a marked increase in surgical intervention, particularly in the use of internal fixation using locking plates. For example, Mattila 2011 reported that the number of plate fixations for these fractures had more than doubled between 2006 and 2008 in Finland. Such clinical interventions (cast immobilisation and surgery followed by immobilisation) are often referred to as 'first definitive' treatments (HSCIC 2015). Although in the following, we refer to these as 'definitive' treatments, this descriptor should not be taken to diminish the status of rehabilitation.

The variety of these 'definitive' treatment options is shown in the Cochrane reviews of conservative (Handoll 2003c) and surgical management of these fractures (Handoll 2003a). The latter review is now replaced by reviews on different surgical methods, such as percutaneous pinning (Handoll 2007), with the exception of internal fixation for which a review is underway (Hoare 2014).

These injuries can result in increased morbidity, with longterm functional impairment, pain and deformity (Edwards 2010; Handoll 2003a; Handoll 2003c). They are also associated with a high incidence of varied complications (McKay 2001); for example, serious complications, such as persistent neuropathies of the median, ulnar or radial nerves, have been reported in one in three patients (Cooney 1980). One major complication is complex regional pain syndrome type 1 (CRPS-1), also referred to as reflex sympathetic dystrophy, algodystrophy, Sudeck's atrophy and shoulder-hand syndrome. Serious cases of CRPS-1 require many months of therapy to alleviate symptoms (pain, tenderness, impairment of joint mobility, swelling, dystrophy, vasomotor instability) (Atkins 1996).

\section{Description of the intervention}

Rehabilitation refers to the overall process of helping people to make the best possible recovery from their injury. The issues surrounding the rehabilitation of patients with a distal radial fracture can be expressed in terms of four basic questions:

- What sort of intervention(s) should be used?

- Who should provide them?

- When and for how long?

- Why?

A variety of interventions are available for use. Advice, patient education and supervision for active and passive mobilisation exercises, therapist-applied mobilisation techniques, continuous passive motion, strengthening exercises, supportive splints, physical methods of pain management such as transcutaneous electrical nerve stimulation (TENS), heat treatment, massage, wound care, manual aids and occupational/home assessment are some of the more common therapeutic methods used to maximise the patient's functional recovery (Collins 1993). A small selection of these, commonly advice and mobility exercises, may be employed on a general basis for all patients. Usually though, interventions are se- 
lected and adapted by clinicians to meet the specific rehabilitation challenges presented by individual patients. Specific rehabilitation programmes of usually physical interventions (primarily exercises) based on a standardised protocol may also be applied; but, because it is not always possible to standardise to the last detail, some flexibility is common. Although drugs may be prescribed, for instance for pain relief, these are not reviewed here.

As well as the doctors, commonly orthopaedic surgeons, providing definitive treatment, other clinicians are often involved in the rehabilitation of patients with these injuries. These other clinicians may be physiotherapists, occupational therapists or nurses, many of whom are specialised in hand and/or upper limb therapy. The distinctions between the activities and roles of these clinicians often overlap and also vary geographically. Generally, physiotherapists aim to help the patient restore or achieve optimal movement and physical function. Occupational therapists share this aim but focus on helping patients to achieve independence in activities of daily living. Nurses may play a varied role, including that of rehabilitation, but plaster cast management and care of surgical wounds would be typical activities. These latter activities are viewed as part of clinical treatment for the purposes of this review.

The issue of when to commence rehabilitation is controversial. Rehabilitation could start as soon as possible after the injury and continue throughout, or rehabilitation could be seen as a subsequent stage in patient management and undertaken after the initial clinical treatment (e.g. cast immobilisation) is over. Therefore, the two key phases for management of these injuries are during initial clinical treatment, which usually involves immobilisation, and post-immobilisation (after plaster cast or external fixator removal). Upon receiving initial treatment, such as fracture reduction and application of a plaster cast, patients are usually given instructions to carry out straightforward exercises. These typically include elevation of the injured arm in the first few days postinjury and exercising of the non-immobilised joints in order to alleviate and/or counter swelling and stiffness. More extensive and intensive rehabilitation intervention is more frequent post-immobilisation; at this stage, limited range and quality of movement, reduced grip strength, and pain are typical reasons for initiating rehabilitation interventions.

The 'why' question mainly concerns the clinical indication for the intervention(s). Our main focus is on studying the effects of rehabilitation interventions on preventing complications associated with the fracture and/or treatment and on optimising functional recovery and achievement of activities required for daily living. Rehabilitation interventions may also be prescribed to treat complications, such as CRPS-1, of these fractures. We acknowledge the difficulties in distinguishing the two situations since there will be overlap but, given our main aim, we noted the reasons for starting or providing the interventions in individual trials. The aims, including intended trial populations, and the primary outcome(s) of individual trials helped us to distinguish between those trials evaluating interventions to resolve or prevent 'problems' and those investigating treatment options for complications. The latter are not included in this review. Similarly excluded are trials primarily investigating interventions for pain relief, acceleration of bone healing, osteoporosis or secondary prevention of fractures.

Editorial feedback for this version of the review prompted consideration of a possible fifth question: 'where'? Although pertinent, we decided against an additional category at this time because 'where should treatment take place?' is generally subsidiary to other questions when set in the context of the care pathway for these fractures. For example, the prime consideration of supervised rehabilitation versus home exercises resolves around the question of who provides rehabilitation (a clinician versus the patient) rather than care in a [hospital] clinic versus exercises at home. While we describe the locations of the interventions tested in the review, so far none of the comparisons is predominantly linked with locality. Nonetheless, this item will be reconsidered before the next version of the review.

\section{How the intervention might work}

The central rationale for rehabilitation has been discussed above. A comprehensive summary of rehabilitation interventions, several of which are usually used in combination, is not provided here. Instead Table 1 provides some illustrative examples of putative mechanisms of a few single rehabilitation interventions, only two of which (advice and instructions, joint mobilisation) are in common use.

\section{Why it is important to do this review}

Distal radius fractures are the, or among the, most common fractures in adults in predominantly white and older populations. These injuries can result in long-term functional impairment and pain, and are also associated with a high incidence and variety of complications. There is considerable variation in the management, including rehabilitation, of these fractures. The previous version of this review concluded that the available evidence from RCTs was insufficient to establish the relative effectiveness of the various interventions used in the rehabilitation of adults with these fractures, and also noted the paucity of the evidence for rehabilitation after surgical treatment (Handoll 2006). This is an update of our systematic review of the evidence for rehabilitation interventions for these fractures.

\section{O B J E C T I VES}

To examine the effects of rehabilitation interventions in adults with conservatively or surgically treated distal radial fractures.

We defined the following specific objectives a priori. 
1. To compare the provision of rehabilitation intervention (of any kind) versus no intervention.

- The rehabilitation intervention could be multicomponent or involve a single modality (e.g. advice for home exercises) and, whilst available to all patients allocated the rehabilitation intervention, its application (use of specific modalities, extent) may vary according to the perceived needs of individual patients.

2. To compare any type of rehabilitation intervention versus any other type of rehabilitation intervention.

- This covers comparisons of different rehabilitation interventions, either in different combinations of rehabilitation modalities or different single modalities. We considered the examination of variation in single modalities to be optional and that the inclusion of trials of any such comparisons was likely to be deferred until the use of the modality had been evaluated.

3. To compare any method (context) of delivering or providing rehabilitation interventions versus any other method of delivering or providing rehabilitation interventions.

- This includes comparisons of supervised therapy versus home exercises, different methods of supervised therapy (e.g. individual versus group instruction), and the frequency and duration of rehabilitation (where rehabilitation is provided to all participants). It also includes comparisons of rehabilitation intervention when delivered by individual professionals with different levels or backgrounds of expertise or training. In the first instance, the various professions were grouped into four categories: doctors; non-specialist therapists; hand or upper limb clinical specialist therapists; and others (e.g. nurses).

For each of these three comparisons we set up separate comparisons according to whether the rehabilitation intervention was provided during immobilisation or other definitive treatment, or post-immobilisation.

\section{METHODS}

\section{Criteria for considering studies for this review}

\section{Types of studies}

We considered any randomised or quasi-randomised (method of allocating participants to a treatment that is not strictly random e.g. by date of birth, hospital record number and alternation) clinical trials of rehabilitation interventions for adults with distal radial fractures.

\section{Types of participants}

Patients of either sex who have completed skeletal growth and who are receiving treatment for a fracture of the distal radius.

The characteristics of the participants included in the trials were noted, particularly: age, gender, employment, type of fracture (especially whether intra-articular or extra-articular), type of treatment, functional and mental status, and comorbidities. We stipulated beforehand that trials evaluating treatment only for patients with established complications, such as wound infection and CRPS-1, would be excluded. Included, however, were trials where the clinical indication, such as wrist stiffness or hand swelling, could be regarded as a 'problem' that may or may not lead to a complication.

\section{Types of interventions}

All RCTs evaluating rehabilitation as part of the conservative or surgical treatment of fractures of the distal radius. Examples of rehabilitation interventions are active (under the control of the patient's own musculature) and passive (an externally applied force, such as by a therapist, is used to move the joint) mobilisation exercises, continuous passive motion devices, strengthening exercises, heat treatment, massage, provision of manual aids, occupational and home assessment, advice and patient education. These interventions may be used in combination or individually, and applied in various ways and by various clinicians.

We proposed in our protocol to exclude trials comparing different techniques, timing (duration, frequency) and intensity of single rehabilitation modalities until the effectiveness of the modality itself had been examined.

We stipulated beforehand that we would exclude all drug trials and trials specifically aimed at analgesia, acceleration of fracture healing, treatment of osteoporosis and secondary prevention of injuries. We also excluded trials evaluating the duration of immobilisation or limited mobilisation through dynamic external fixation; these are covered in other reviews (Handoll 2003a; Handoll 2003c).

\section{Types of outcome measures}

For presentation purposes in this review update (2015), we have retained our previous structuring of the outcomes in four categories: functional outcomes (including impairment), clinical outcomes, resources, and others. We have also identified the primary outcomes that guided our interpretation of the evidence. These outcomes will be presented in 'Summary of findings' tables should the evidence be sufficient to warrant these in future updates. We will set out a full list of outcomes for inclusion in 'Summary of findings' tables in the next version of the review.

1. Functional outcomes (including impairment)

- Range of movement (digits, wrist, forearm, elbow and shoulder mobility), pain, grip strength, activities of daily living (including return to previous employment). Also included are 
patient functional assessment instruments such as Short Form 36 (SF-36), the Disability of the Arm, Shoulder and Hand questionnaire (DASH) and the Patient-Rated Wrist Evaluation (PRWE) (MacDermid 2000).

- Some people have questioned the inclusion of some of the measures listed in this category. We acknowledge that range of motion, grip strength and pain might be classed as measures of impairment and might be considered to be clinical outcomes rather than functional ones. We nonetheless retain these in the functional outcome category for consistency with the literature on these fractures.

2. Clinical outcomes

- Residual soft tissue swelling, early and late complications including complex regional pain syndrome type 1 (CRPS-1).

3. Resources

- Number of outpatient attendances, clinician consultations and other costs.

4. Others

- Malunion, cosmetic appearance, compliance and patient satisfaction.

\section{Primary outcomes}

In interpreting the evidence from the included trials we gave emphasis to whether the following data were reported.

1. Functional outcomes: patient-reported measures of wrist and hand function (e.g. PRWE) and upper limb function (e.g. DASH).

2. Activities of daily living and health-related quality of life scores (e.g. EuroQol (EQ-5D); Short-Form 36 (SF-36) and Short-Form 12 (SF-12; Ware 1996).

3. Serious adverse events (e.g. CRPS-1), and need for substantive treatment, such as extensive physiotherapy.

We based our judgement of clinically important between-group mean differences in measures of pain and function using the following minimal clinically important differences (MCIDs); alternative MCIDs with sources are listed after the main selected item in bold.

- DASH (0 to 100: worst outcome): 10 (Sorensen 2013). Alternatives: 17.1 (Schmitt 2004); 15 recommended in DASH/ QuickDASH.

- QuickDASH: 14 (Sorensen 2013). Alternatives: 16 suggested in DASH/QuickDASH.

- PRWE: 11.5 (Walenkamp 2015). Alternatives: 14

(Sorensen 2013); 24 (Schmitt 2004).

\section{Search methods for identification of studies}

\section{Electronic searches}

We searched the Cochrane Bone, Joint and Muscle Trauma Group Specialised Register (12 January 2015), the Cochrane Central Register of Controlled Trials (CENTRAL; 2014 Issue 12), MEDLINE (1966 to January Week 1 2015), MEDLINE In-Process \& Other Non-Indexed Citations (9 January 2015), EMBASE (1988 to 2015 Week 2), Cumulative Index to Nursing and Allied Health Literature (CINAHL) (1982 to 12 January 2015), Allied and Complementary Medicine (AMED) (1985 to 12 January 2015), the Latin American and Caribbean Health Sciences Database (LILACS) (13 January 2015), PEDro (the Physiotherapy Evidence Database) (accessed 14 February 2012) and OTseeker (the Occupational Therapy Systematic Evaluation of Evidence Database) (accessed 13 February 2013). We did not apply any language restrictions.

In MEDLINE, we combined subject-specific terms with the sensitivity-maximizing version of the Cochrane Highly Sensitive Search Strategy for identifying randomised trials (Lefebvre 2011). Search strategies for the CENTRAL, MEDLINE, EMBASE, CINAHL, AMED, PEDro, LILACS and OTseeker are shown in Appendix 1. Details of the search strategies used for previous versions of the review are given in Handoll 2006.

We also searched the WHO International Clinical Trials Registry Platform Search Portal (WHO ICTRP) and ClinicalTrials.gov to identify ongoing and recently completed trials (February 2015) (see Appendix 1).

\section{Searching other resources}

We checked reference lists of articles. We searched abstracts of the American Academy of Orthopaedic Surgeons (AAOS) annual meeting (2014), the American Orthopaedic Trauma Association (AOTA) annual meetings (2012 to 2014), the Bone and Joint Journal (BJJ) Orthopaedic Proceedings (6 June 2015), the British Society for Surgery of the Hand (BSSH) meetings (2007 to 2014) and the British Trauma Society (BTS) annual scientific meeting (2014).

\section{Data collection and analysis}

\section{Selection of studies}

Pairs of review authors (current and former) independently screened search results and assessed potentially eligible studies for inclusion. Any disagreement was resolved through discussion. Titles of journals, and names of authors or supporting institutions were not masked at any stage. We recorded the selection process in a PRISMA flow diagram (Moher 2009).

\section{Data extraction and management}

Pairs of review authors (current and former) independently extracted data for six of the newly included studies. Data extraction 
was performed by one review author $(\mathrm{HH})$ for the other five studies.

Where necessary, we contacted trialists for additional details of trial methodology and results.

\section{Assessment of risk of bias in included studies}

Pairs of review authors (current and former) independently assessed risk of bias for newly included trials, without masking of the source and authorship of the trial reports, and one author $(\mathrm{HH})$ assessed risk of bias for trials that had been assessed in previous versions of the review. Between rater and between versions $\mathrm{HH}$ checked consistency in assessment at data entry. All inter-rater differences were resolved by discussion. We used the tool outlined in the Cochrane Handbook for Systematic Reviews of Interventions (Higgins 2011b). This tool incorporates assessment of randomisation (sequence generation and allocation concealment), blinding (of participants and treatment providers, and outcome assessment), completeness of outcome data, selection of outcomes reported and other sources of bias. We considered 'subjective' outcomes (e.g. patient-rated functional outcome scores, pain) and 'objective' outcomes (e.g. functional impairment, complications) separately in our assessment of blinding (performance bias). In our assessment of blinding (detection bias), we further split objective outcomes into those of functional impairment (e.g. grip strength) and the rest (e.g. complications). We considered short-term (up to three months follow-up) and longer-term (three months or longer follow-up) outcomes in our assessment of completeness of outcome data. We assessed two additional sources of bias: bias resulting from major imbalances in key baseline characteristics (e.g. age, gender, type of fracture, type of definitive treatment); and performance bias, particularly 'differential expertise' bias resulting from lack of comparability in clinician's experience with the interventions under test.

Additionally, we assessed five other aspects of trial design and reporting that would help us judge the applicability of the trial findings. The five aspects were: definition of the study population; description of the interventions; definition of primary outcome measures; relevance of outcome measurement; and length of follow-up.

\section{Measures of treatment effect}

For each trial, we calculated risk ratios (RRs) and 95\% confidence intervals (CIs) for dichotomous outcomes, and mean differences (MDs) and 95\% CIs for continuous outcomes. We intended to use standardised mean differences rather than MDs when pooling data from continuous outcome measures based on different scoring schemes.

\section{Unit of analysis issues}

We were alert to potential unit of analysis issues arising from inclusion of participants with bilateral fractures, and presentation of outcomes, such as total complications, by the number of outcomes rather than participants with these outcomes. No study reported on patients with bilateral fractures. We avoided the second described unit of analysis problem, mainly by reporting on the incidences of individual complications.

\section{Dealing with missing data}

We contacted trialists for missing information, including for denominators and standard deviations. We performed intention-totreat analyses where possible. Where there were missing standard deviations, we calculated these from other data (standard errors, 95\% CIs, exact P values) where available. We did not impute missing data including standard deviations.

\section{Assessment of heterogeneity}

We planned to assess heterogeneity for pooled data from comparable trials by visual inspection of the analyses, along with consideration of the $\mathrm{Chi}^{2}$ test for heterogeneity (we considered this to be statistically significant at $\mathrm{P}<0.10$ ) and the $\mathrm{I}^{2}$ statistic (Higgins 2003). The main quantitative assessment of heterogeneity was to have been based on the $\mathrm{I}^{2}$ statistic where the following interpretation from the Cochrane Handbook for Systematic Reviews of Interventions would have been used: $0 \%$ to $40 \%$ might not be important; $30 \%$ to $60 \%$ may represent moderate heterogeneity; $50 \%$ to $90 \%$ may represent substantial heterogeneity; and $75 \%$ to $100 \%$ considerable heterogeneity (Deeks 2011).

\section{Assessment of reporting biases}

In the event that a meta-analysis of primary outcomes includes more than 10 studies in a future update, we will consider generating a funnel plot to explore the potential for publication bias.

\section{Data synthesis}

Where available and appropriate, we presented quantitative data, both dichotomous and continuous, for outcomes listed in the inclusion criteria. We stipulated beforehand that results of comparable groups of trials would be pooled using the fixed-effect model and $95 \%$ CIs. Where there was significant heterogeneity between the results of individual trials, and when considered appropriate, the results of the random-effects model were to be viewed and presented instead of those from the fixed-effect model. However, data pooling was only possible in one case but was abandoned given the clear heterogeneity in the results of the two trials involved. Generally, we presented the results for the final follow-up time for which they were available. However, we presented limited interim results from some trials. (We were mindful also of the intention stated in our protocol that we would note interim results where a marked and important difference in the timing of recovery had occurred). 


\section{Subgroup analysis and investigation of heterogeneity}

We planned, but did not perform, subgroup analyses by definitive treatment (surgery versus non-surgical treatment), age (younger adults, older adults; provisional threshold of 65 years), gender, employment status, type of fracture (primarily extra-articular versus intra-articular fractures), comorbidities, and prior functional and mental status. To test whether the subgroups are statistically significantly different from one another, we planned to inspect the overlap of CIs and perform the test for subgroup differences available in Review Manager 5 (RevMan 2014).

\section{Sensitivity analysis}

We planned, but did not perform, sensitivity analyses examining various aspects of trial and review methodology, including the effects of missing data, the inclusion of studies at high or unclear risk of bias (primarily, selection bias with reference to allocation concealment), outcome assessor blinding, inclusion of studies only reported in abstracts and using fixed-effect versus random-effects models for pooling.

\section{'Summary of findings' tables and quality assessment of the evidence}

In view of the lack of pooled data, we decided against producing standard 'Summary of findings' tables for any of the comparisons tested so far in the review. However, we produced tables that presented for each comparison, grouped under the main comparisons, the underlying question (participants/interventions/comparisons/outcomes (PICO)), a summary of the findings, an overall assessment of the quality of the evidence available for the key reported outcomes based on the GRADE approach (see section 12.2, Schunemann 2011), and comments that included a statement on applicability.

\section{RE S U L T S}

\section{Description of studies}

\section{Results of the search}

We updated the search from January 2005 to January 2015. We screened a total of 1844 records from the following databases: Cochrane Bone, Joint and Muscle Trauma Group Specialised Register (13 records); CENTRAL (297), MEDLINE (304), EMBASE (223), CINAHL (195), AMED (11), PEDro (49), LILACS (308), OTseeker (52), the WHO ICTRP (240) and ClinicalTrials.gov (152). We also identified four potentially eligible studies from other sources (abstracts of the AAOS annual meeting 2014 (241), the AOTA annual meetings (2012 to 2014) (96), the BJJ Orthopaedic Proceedings (39; see Appendix 1) and the BTS annual scientific meeting 2014 (37). We identified no potentially eligible trials from our search of BSSH meeting abstracts.

The search update resulted in the identification of 30 new studies (published in 42 reports). Of these, we included 10 trials (Bighea 2013; Brehmer 2014; Challis 2007; Jongs 2012; Knygsand-Roenhoej 2011; Krischak 2009; Kuo 2013; Lazovic 2012; Magnus 2013; Souer 2011), excluded seven studies (ACTRN12606000160538; Ayhan 2014; Bünger 2011; Kingston 2014; Lohstrater 2006; Naik 2007; Wang 2012), placed eight in ongoing trials (ACTRN12612000118808; JPRN-UMIN000015003; NCT01118715; NCT01394809; NCT01518179;

NCT01693094; NCT01921062; NCT02015468) and five await classification (NCT00816998; NCT01262807; NCT01589627; Oken 2011; Schmidt 2013). Three trials listed as ongoing trials in the previous review changed status. Upon publication of a full report, one previously ongoing trial is now included (Kay 2008; formerly Kay 2003). Of the other two previously ongoing trials, one is excluded (Woodbridge 2003) and one is awaiting classification (McPhate 1998). A full report published in 2006 of an already excluded trial (Haren 2004) confirmed its status.

Overall, there are now 26 included studies, 22 excluded studies, eight ongoing trials and seven studies awaiting assessment.

A summary of the study selection process is shown in Figure 1.

Rehabilitation for distal radial fractures in adults (Review) 
Figure I. Study flow diagram for updated review

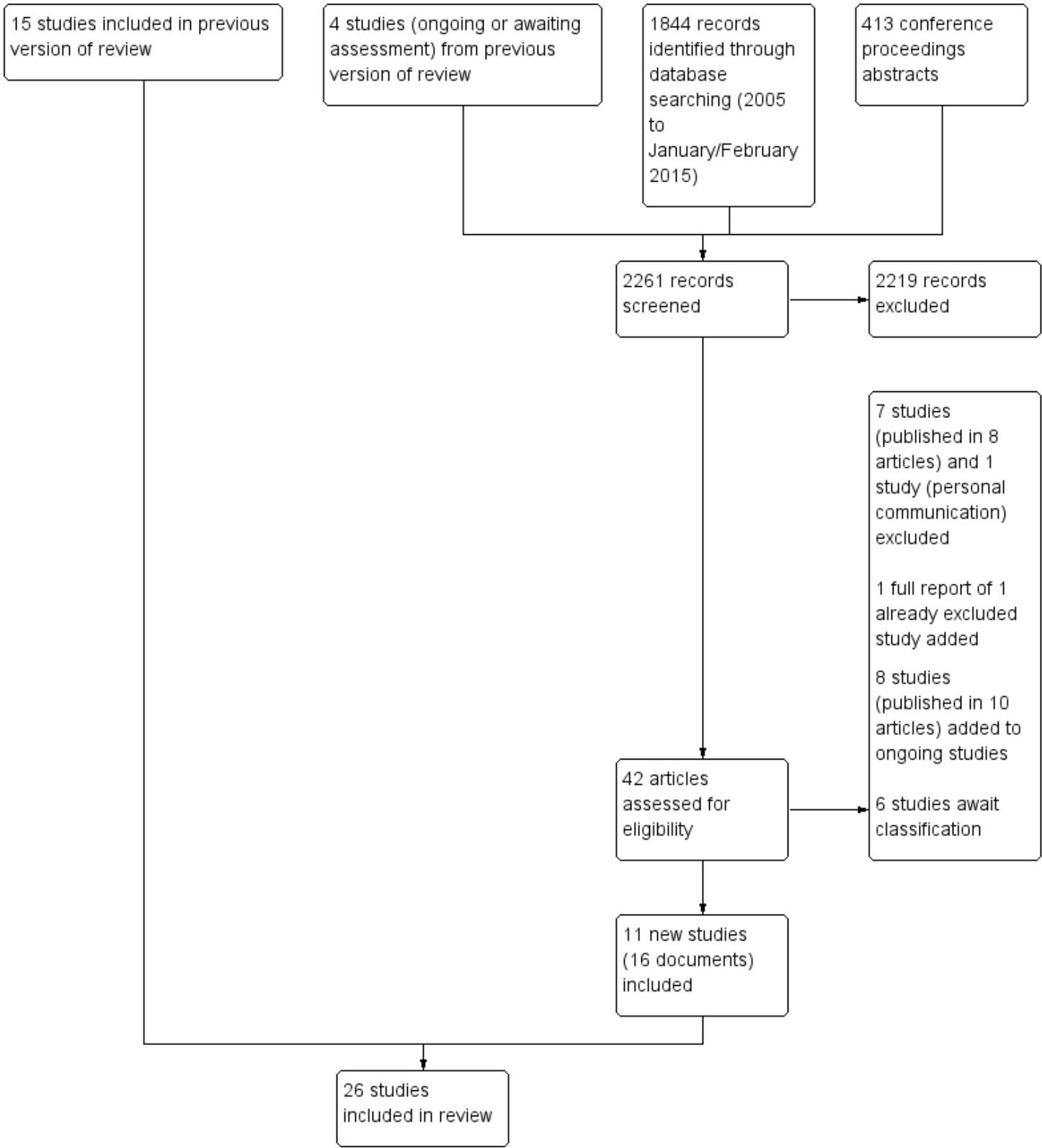

Rehabilitation for distal radial fractures in adults (Review) 


\section{Included studies}

Most of the included studies were fully reported in medical journals. Reports of three trials (Bache 2001; Bighea 2013; Rozencwaig 1996) are only available as abstracts, although a still unpublished report for Bache 2001 was prepared for journal publication (September 2001). The full report of Cooper 2001 is only available as a Master's thesis. We received additional information from trialists of 12 trials, including an interim draft for Bache 2001. Translations were obtained for the two trial reports in Danish (Gronlund 1990; Svensson 1993).

Further details of the individual studies are provided in the Characteristics of included studies tables.

\section{Design}

Nineteen trials were RCTs and three trials were quasi-RCTs (Basso 1998; Lazovic 2012; Rozencwaig 1996). The absence of information on the methods of randomisation for four trials claiming to be randomised meant that we are uncertain regarding the method of sequence generation in these trials (Bighea 2013; Knygsand-Roenhoej 2011; Pasila 1974; Toomey 1986).

\section{Sample sizes}

The included trials were usually small, with sample sizes ranging from seven (Rozencwaig 1996) to 135 (Pasila 1974).

\section{Setting}

The periods over which individual trials were conducted spanned five decades from the early 1970s (Pasila 1974) onwards. Although the provision of care took place in several local sites for some trials, all were co-ordinated from single centres within one of 11 countries (Australia (7 trials), Canada (2 trials), Denmark (4 trials), Finland (1 trial), Germany (1 trial), Hong Kong (1 trial), Romania (1 trial), Serbia ( 1 trial), Taiwan ( 1 trial), UK (4 trials), USA ( 3 trials)).

\section{Participants}

The study populations are summarised in Table 2.

The 26 included studies recruited a total of 1269, mainly female and older, patients. Aside from Bighea 2013 and Rozencwaig 1996, which provided no information on gender or age, all trials recruited more female than male participants; the proportion of females ranged from 58\% (Basso 1998) to 100\% (Lazovic 2012; Magnus 2013; Svensson 1993). Where provided, the median or mean ages of trial populations ranged between 48 years (KnygsandRoenhoej 2011) to 76 years (Watt 2000). The youngest participant (15 years) appeared in Basso 1998 and the oldest (93 years) in
Gronlund 1990. Lower age limits were set by 18 trials: 15 years (Basso 1998); 16 years (Cooper 2001; Pasila 1974); 18 years ( Brehmer 2014; Challis 2007; Jongs 2012; Knygsand-Roenhoej 2011; Krischak 2009; Maciel 2005; Souer 2011); 35 years (Taylor 1994); 45 years (Gronlund 1990:); 50 years (Bache 2001; Kuo 2013; Magnus 2013); and 55 years (Lazovic 2012; Svensson 1993; Wakefield 2000). An upper limit of 65 years was applied in Pasila 1974 and 85 years in Brehmer 2014.

Fracture type was broadly defined as either distal radius fracture in 17 trials or Colles' fracture in eight trials. Bighea 2013 referred only to osteoporotic wrist fracture. More details of fracture types were available in several trials. Participants of 15 trials were initially treated conservatively, involving plaster cast immobilisation. Initial treatment was surgery (external fixation or internal fixation) for all participants in five trials (Brehmer 2014; Krischak 2009; Kuo 2013; Rozencwaig 1996; Souer 2011). Initial treatment was either surgery (percutaneous pinning, external fixation or internal fixation) or plaster cast alone in six trials (Jongs 2012; Kay 2000; Kay 2008; Knygsand-Roenhoej 2011; Maciel 2005; Magnus 2013). The rehabilitation intervention or interventions under test were started during definitive treatment in seven trials (Challis 2007; Cooper 2001; Gronlund 1990; Kuo 2013; Lazovic 2012; Magnus 2013; Pasila 1974), and post-immobilisation or after definitive treatment in the other 19 trials.

Details of the inclusion and exclusion criteria of the individual studies are provided in the Characteristics of included studies tables. Table 2 summarises these in terms of the exclusion of people with complications, such as CRPS-1, and comorbidities or functional dependency. Where sufficient information was available to judge, the populations of 13 trials were limited to those without serious complications, often CRPS-1, or comorbidities or both of these (Bache 2001; Basso 1998; Cheing 2005; Cooper 2001; Gronlund 1990; Krischak 2009; Kuo 2013; Lazovic 2012; Maciel 2005; Magnus 2013; Svensson 1993; Toomey 1986; Wakefield 2000). Populations would have been limited by suitability of surgery, involving internal fixation, in Brehmer 2014 and Souer 2011. The study populations of two trials clearly included patients with or without complications ( Kay 2000; Kay 2008); this also may have applied in two other trials (Maciel 2005; Watt 2000). Two trials limited their populations to those with complications, these being wrist contracture in Jongs 2012 and subacute oedema in Knygsand-Roenhoej 2011.

\section{Interventions}

All trials had two intervention groups with the exception of Cheing 2005, which had four intervention groups. Table 3 presents a summary of the rehabilitation interventions, the care providers, when the interventions were started, where they were provided and

Rehabilitation for distal radial fractures in adults (Review) 
for how long. Comments mainly describing treatment provided to all trial or all control group participants of individual trials are also given. The following summary presents the trials according to the comparisons implied in the review objectives, split by the timing of the intervention.

\section{Comparisons}

\section{(1) Rehabilitation intervention versus no intervention}

Nineteen trials fell into this category, six of which (Challis 2007; Cooper 2001; Gronlund 1990; Kuo 2013; Lazovic 2012; Magnus 2013) started during the definitive treatment period. Eight trials (Bache 2001; Christensen 2001; Cooper 2001; Gronlund 1990; Kay 2008; Kuo 2013; Maciel 2005; Wakefield 2000) evaluated a multi-component intervention, whereas the other 11 (Basso 1998; Challis 2007; Cheing 2005; Jongs 2012; Kay 2000; Lazovic 2012; Magnus 2013; Rozencwaig 1996; Svensson 1993; Taylor 1994; Toomey 1986) examined single interventions. Cheing 2005 also examined the combined effect of two single interventions.

\section{(1a) Rebabilitation started during the definitive treatment period}

Cooper 2001 compared "early therapeutic intervention", involving weekly contact with a member of the hand therapy team, started within four days of injury and plaster cast application, versus no intervention in 17 people. All participants received instructions for home exercises during plaster cast immobilisation and an individualised home programme of exercises post-immobilisation with a criteria-based offer to attend a hand therapy group. Gronlund 1990 compared the provision of "occupational therapy" one to three days after the application of a plaster cast to no provision in 40 participants. All participants received instructions for exercises and other information after their initial treatment and, if judged necessary, were referred to occupational therapy after the plaster cast removal.

Challis 2007 compared cyclic pneumatic soft tissue compression using an inflatable cuff placed under the plaster cast versus usual care during the five-week immobilisation period in a full forearm plaster cast in 21 people. All participants received instruction for hand exercises during cast immobilisation and were given an exercise programme for four weeks after cast removal. Kuo 2013 compared a progressive early digit mobilisation programme, involving three 45-minute sessions per week delivered by an occupational therapist, versus usual care during six weeks of external fixation in 22 people. Although not confirmed by Kuo 2013, the components of usual care such as advice for arm elevation were likely to have been provided to both groups. Lazovic 2012 compared 10 sessions of pulsed electromagnetic field therapy over two weeks versus no therapy control during cast immobilisation in 60 participants. Magnus 2013 evaluated a 'cross-education' intervention involving a progressive home-based programme of strength training of the non-fractured hand. All 51 participants, 11 of whom had surgical fixation, were provided with a standard rehabilitation programme of home exercises for the fractured hand, starting with range of motion exercises of uninvolved joints during six weeks of forearm cast immobilisation.

\section{(1b) Rehabilitation started post-immobilisation}

Five trials evaluated the provision of routine therapy, ranging from a single session to a prolonged programme, following plaster cast removal. Kay 2008 compared a single session of physiotherapy, primarily advice and instructions for a home exercise programme, versus no intervention in 56 participants. Christensen 2001 compared the provision of around twice weekly "occupational therapy", until the therapist perceived a lack of progress, with no provision in 32 participants. All participants received instructions from an occupational therapist for exercises to be performed on a thricedaily basis at home. Bache 2001 and Wakefield 2000 compared the provision of routine physiotherapy with no provision in 98 and 96 participants, respectively. The content of the physiotherapy was at the discretion of the physiotherapist in both trials; however, there was restriction to a set of agreed modalities in Bache 2001. All participants received instructions for home exercises from a physiotherapist within one week of plaster removal in Bache 2001, and at the fracture clinic on the same day as plaster cast removal in Wakefield 2000. Maciel 2005 compared the regular attendance of "activity-focussed" physiotherapy for up to six weeks with the option of a single advice session from a physiotherapist solely to clarify home exercises in 41 of the 45 people recruited into the trial. All participants of Maciel 2005 were taught home exercises and received information from a physiotherapist on the day of cast removal.

Rozencwaig 1996 investigated the addition of continuous passive motion to occupational therapy versus occupational therapy alone following external fixation in seven participants.

Cheing 2005 tested the application of pulsed electromagnetic field (PEMF) or ice, or both for 30-minute sessions over five consecutive days in 83 participants. The four intervention groups were: PEMF plus ice pack; sham PEMF plus ice pack; PEMF; sham PEMF. All participants received a "standard" home exercise programme.

Two studies evaluated passive mobilisation given post-immobilisation by experienced physiotherapists. Kay 2000 compared a sixweek course of passive mobilisation with no passive mobilisation in 40 participants, 13 of whom had been initially treated with pins and plaster. All participants received initial physiotherapy including advice and instructions for home exercises and were monitored for progression with correction if necessary. Taylor 1994 compared five minutes of passive mobilisation with soft tissue massage (sham treatment) within twice-weekly treatment sessions at the physio- 
therapy department in 30 participants. All participants received advice and instruction for home exercises.

Svensson 1993 evaluated 20 minutes of intermittent pneumatic compression before each of nine sessions of occupational therapy; these were started around 25 days following plaster cast removal in 43 participants who had been referred to the rheumatological department.

Basso 1998 compared the active versus sham application of low frequency, long-wave ultrasound to the back of the affected wrist for five minutes following plaster cast removal in 38 participants. All participants were given instructions to move their hand as much as possible. Physiotherapy was provided only if "hand function was poor".

Toomey 1986 compared forearm immersion in a whirlpool with the wrapping of the forearm in two towels during the first 15 minutes of 12 sessions of physiotherapy, scheduled over six weeks following plaster cast removal, in at least 24 participants. In this review, participants treated with two towels are considered as a no intervention or control group.

Jongs 2012 investigated the use of dynamic wrist extension splints, worn up to six hours on a daily basis for eight weeks, in 40 participants with wrist contracture who had been referred to physiotherapy at least 10 weeks from their fracture.

(2) One rehabilitation intervention versus another rehabilitation intervention

\section{(2a) Rebabilitation started during the definitive treatment} period

No trial was available.

\section{(2b) Rehabilitation started post-immobilisation}

Watt 2000 compared the routine referral for physiotherapy with the provision by an orthopaedic surgeon or registrar of a home exercise sheet and simple home instructions at an outpatient clinic following plaster cast removal in 18 participants. The content of the physiotherapy was at the discretion of the therapist but always included active exercises, instructions for a home exercise programme and advice; passive joint mobilisation by the therapist was used in $47 \%$ of the treatments.

One of the comparisons undertaken in Cheing 2005 was that of pulsed electromagnetic field treatment versus ice in 44 participants. All participants received a home exercise programme.

Knygsand-Roenhoej 2011 compared two types of treatment for subacute oedema: modified "manual edema mobilization" (MEM) versus a 'traditional' oedema technique in 30 participants.
(3) Any method (context) of delivering or providing rehabilitation interventions versus any other method of delivering or providing rehabilitation interventions

\section{(3a) Rebabilitation started during the definitive treatment period}

Pasila 1974 compared supervised therapy at the physical medicine department with home exercises; both were started after cast application treatment in 135 participants. The same oral and written instructions for exercising non-involved joints were provided to participants by a physiotherapist in the supervised group, and the surgeon or physician in the control group. No other physiotherapy was carried out.

\section{(3b) Rehabilitation started post-immobilisation}

Three trials compared supervised exercise therapy versus a home exercise programme. Bighea 2013 compared 20 sessions of physiotherapy (galvanic bath then exercise programme) versus a home exercise programme over four weeks; both started after four weeks of cast immobilisation. Krischak 2009 compared 12 sessions of physiotherapy versus a progressive home exercise programme over six weeks; both were started one week after surgery involving open reduction and volar plate fixation in 48 participants. The physiotherapy was at the discretion of the physiotherapist. After instructions, the home exercise group received an exercise guidance book and training diary. This trial is considered to start post-immobilisation because the post-surgical splint was retained for one week after the start of therapy and was removed for therapy. Souer 2011 compared occupational therapy versus coaching for a programme of home exercises in 94 patients at the first post-operative visit to the surgeon's office after volar plate fixation. There was no mention of use of splintage in the occupational therapy group but participants of the home exercise group were provided with a wrist splint for use until they had recovered full forearm and finger motion. Again, this trial fits better into the post-immobilisation category.

One trial (Brehmer 2014) with 81 participants investigated accelerated (started at two weeks) versus usual rehabilitation (started at six weeks) after volar plate fixation. Although the duration of immobilisation differed between the two groups, this trial met the review inclusion criteria because the focus was on timing of rehabilitation.

\section{Outcome measurement}

Details of the follow-up schedules and the outcomes measured in individual studies are provided in the Characteristics of included studies tables. As summarised in Table 4, length of follow-up ranged from a few days (Cheing 2005; Lazovic 2012) to nine 
months (Christensen 2001). Also captured in Table 4 is an assessment of the adequacy of the description of the main outcomes reported in each trial and whether these were relevant. Relevance was judged primarily in terms of whether there was subjective reporting by the trial participants of their function. Thirteen trials, most of which were included in this update, reported patientreported outcome measures (Bache 2001; Bighea 2013; Brehmer 2014; Cooper 2001; Jongs 2012; Kay 2008; Knygsand-Roenhoej 2011; Krischak 2009; Kuo 2013; Lazovic 2012; Maciel 2005; Magnus 2013; Souer 2011). Notably, this list features 10 of the 11 studies newly included in this update, seven of which reported the Patient-Rated Wrist Evaluation score (PRWE).

\section{Excluded studies}

Details and justification for the exclusion of 22 studies are presented in the Characteristics of excluded studies tables. The reasons for exclusion relate to study design, focus, status and reporting. Six studies were not randomised or quasi-randomised (Can 2001; Hunt 2001; Jarvis 2001; Nikolova 1969; Oskarsson 1997; Ramesh 1998). Two very small trials involved a single-subject study design, which is inappropriate for this review (Coyle 1998; Neeman 1988). As reflected in the selection of the intervention, participants, outcomes or combinations of these, the focus of eight studies was outside the scope of this review (Ayhan 2014; Haren 2000; Haren 2004; Kingston 2014; Pasila 1980; Rodrick 2004; Wang 2012; Zhang 2005). Examples of this category are the three trials that focussed on interventions for treating oedema after fracture fixation that reported only on this outcome (Haren 2000; Haren 2004; Wang 2012). We excluded six trials because of trial status or inadequate reporting or a combination of these (ACTRN12606000160538: no indication that this started; Bünger 2011: under-recruiting pilot study reporting results for just two patients; Lohstrater 2006: very poorly reported interim analysis; Naik 2007 and Schwartz-Jensen 2002: too poorly reported with no further details obtainable; Woodbridge 2003: no report available).

\section{Ongoing studies}

Details of the eight ongoing studies, all of which appear in trial registers, are presented in the Characteristics of ongoing studies. When rechecked on 6 June 2015, one trial is listed as 'not yet recruiting' (JPRN-UMIN000015003: aim 56 participants); three trials are 'currently recruiting participants' ( NCT01118715: aim 460 participants; NCT01518179: aim 120 participants; NCT01921062: aim 52 participants) and one trial is 'enrolling participants by invitation only (NCT01693094: aim 126 participants, only some of whom will have distal radius fracture). One trial is 'active, not recruiting' ( NCT02015468: aimed 120 participants); and two are 'completed' (ACTRN12612000118808: 30 participants; NCT01394809: 27 participants). Two trials are testing the use of compression gloves (NCT01118715; NCT01518179) and two are testing the use of motor cognitive training (NCT01921062; NCT01394809). The four other trials are testing four other interventions: exercise (ACTRN12612000118808); occupational therapy (JPRNUMIN000015003); use of decision aids (NCT01693094); early mobilisation and physiotherapy (NCT02015468).

\section{Studies awaiting classification}

Details of the seven small studies awaiting assessment, each of which made a different comparison, are presented in the Characteristics of studies awaiting classification tables. Trial registration documents only are available for three trials ( NCT00816998; NCT01262807; NCT01589627), and incomplete reports in the form of journal or conference abstracts are only available for two other trials (Duvoric 2005; McPhate 1998). Clarification on study methods is required for the two remaining studies, both of which are reported in full articles (Oken 2011; Schmidt 2013).

\section{Risk of bias in included studies}

The risk of bias judgements on 12 items for the individual trials are summarised in Figure 2 and described in the 'Risk of bias' tables in the Characteristics of included studies tables. A '(+)' judgement means that the authors considered there was a low risk of bias associated with the item, whereas a '(-)' means that there was a high risk of bias. Many assessments resulted in an unclear '(?)' verdict; this reflected a lack of information upon which to judge the item or absence of data for a specific outcome category, such as absence of subjective outcomes or lack of longer-term followup (see Figure 3). 
Figure 2. Risk of bias summary: review authors' judgements about each risk of bias item for each included study.

\begin{tabular}{|c|c|c|c|c|c|c|c|c|c|c|c|c|}
\hline & 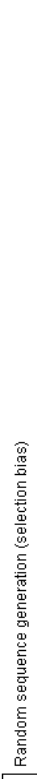 & 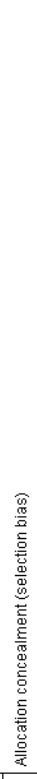 & 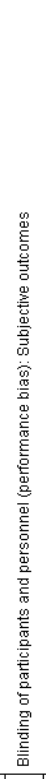 & 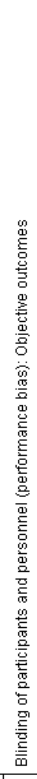 & 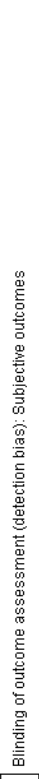 & 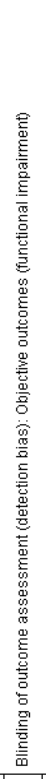 & 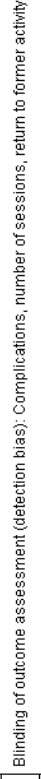 & 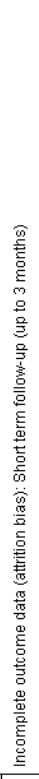 & 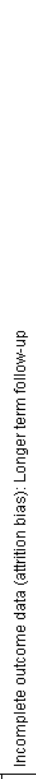 & 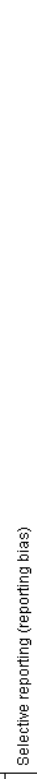 & 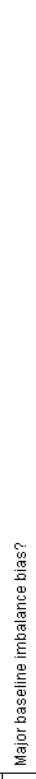 & 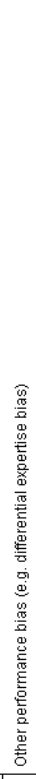 \\
\hline Bache 2001 & $\odot$ & $?$ & $\theta$ & $\theta$ & $?$ & $?$ & $\odot$ & $?$ & $?$ & $?$ & $?$ & $\odot$ \\
\hline Basso 1998 & ? & ? & $?$ & $\odot$ & $?$ & + & - & $?$ & $?$ & $?$ & $?$ & $?$ \\
\hline Bighea 2013 & $?$ & $?$ & $\theta$ & ○ & $\theta$ & $\theta$ & $?$ & $?$ & $?$ & $\theta$ & $?$ & $?$ \\
\hline Brehmer 2014 & $\odot$ & $\odot$ & $\Theta$ & $\theta$ & $\theta$ & $\theta$ & ○ & $?$ & $\Theta$ & $\odot$ & $?$ & $\odot$ \\
\hline Challis 2007 & $\odot$ & $\odot$ & $?$ & $\theta$ & $?$ & $\odot$ & $?$ & $?$ & $?$ & $?$ & $\odot$ & $\odot$ \\
\hline Cheing 2005 & $?$ & $?$ & $?$ & $?$ & $?$ & $?$ & $\odot$ & $?$ & $?$ & $?$ & $?$ & $\odot$ \\
\hline Christensen 2001 & $?$ & $\odot$ & $?$ & $\theta$ & $?$ & $\odot$ & $\odot$ & $?$ & $?$ & $?$ & $\odot$ & $\odot$ \\
\hline Cooper 2001 & $\odot$ & $\odot$ & $\odot$ & ○ & ? & O & - & + & $?$ & + & ○ & $?$ \\
\hline Gronlund 1990 & $\odot$ & $\odot$ & 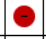 & ○ & $?$ & $?$ & + & $?$ & $?$ & $?$ & $?$ & $?$ \\
\hline Jongs 2012 & $\odot$ & $\odot$ & $\theta$ & $\theta$ & ○ & - & $?$ & $?$ & $?$ & $\odot$ & $?$ & $\odot$ \\
\hline Kay 2000 & $\odot$ & 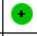 & - & $\theta$ & $?$ & $\odot$ & $\odot$ & $\odot$ & $?$ & $\odot$ & $\odot$ & $\odot$ \\
\hline Kay 2008 & $\odot$ & $\odot$ & - & ○ & ? & $\odot$ & $?$ & O & $?$ & $?$ & $\odot$ & $?$ \\
\hline Knygsand-Roenhoej 2011 & $?$ & $?$ & ○ & $\theta$ & $?$ & + & $?$ & $?$ & + & $?$ & + & $?$ \\
\hline Krischak 2009 & $?$ & $?$ & $\odot$ & $\theta$ & 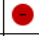 & $\theta$ & $\odot$ & $?$ & $?$ & $?$ & $\odot$ & $?$ \\
\hline Kuo 2013 & $?$ & $?$ & $\theta$ & ○ & $\theta$ & $?$ & $\odot$ & $\odot$ & $?$ & + & $?$ & $\odot$ \\
\hline Lazovic 2012 & ○ & ○ & - & ○ & ? & 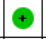 & $\odot$ & $?$ & $?$ & $?$ & $?$ & $\odot$ \\
\hline Maciel 2005 & $\odot$ & + & - & $\theta$ & $\theta$ & + & $\odot$ & $?$ & $?$ & $?$ & $?$ & $?$ \\
\hline Magnus 2013 & $\odot$ & + & $\Theta$ & $\odot$ & $\theta$ & $\odot$ & $\odot$ & $\theta$ & 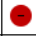 & $?$ & $?$ & $?$ \\
\hline Pasila 1974 & $?$ & $?$ & $\theta$ & $\theta$ & ○ & $\theta$ & $?$ & $\theta$ & $?$ & $\theta$ & $?$ & 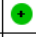 \\
\hline Rozencwaig 1996 & ○ & ○ & $?$ & ○ & $?$ & C & ? & $?$ & $?$ & O & $?$ & $?$ \\
\hline Souer 2011 & $\odot$ & $?$ & ○ & $?$ & $\theta$ & $?$ & $\odot$ & $\odot$ & $?$ & + & + & $?$ \\
\hline Svensson 1993 & $?$ & $?$ & - & $\theta$ & 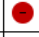 & - & $?$ & $\theta$ & $?$ & $?$ & $?$ & + \\
\hline Taylor 1994 & $?$ & ○ & $?$ & $\theta$ & $?$ & O & ○ & $\odot$ & $?$ & $?$ & $?$ & $\odot$ \\
\hline Toomey 1986 & $?$ & $?$ & - & $\theta$ & $\theta$ & † & $?$ & $?$ & $?$ & $?$ & + & † \\
\hline Wakefield 2000 & $\odot$ & $\odot$ & - & $\theta$ & $\theta$ & $?$ & 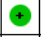 & $?$ & ? & $\theta$ & + & $\odot$ \\
\hline Watt 2000 & $\odot$ & $\odot$ & $?$ & $\theta$ & $?$ & $\odot$ & ○ & $?$ & $?$ & + & $\odot$ & $?$ \\
\hline
\end{tabular}


Figure 3. Risk of bias graph: review authors' judgements about each risk of bias item presented as percentages across all included studies.

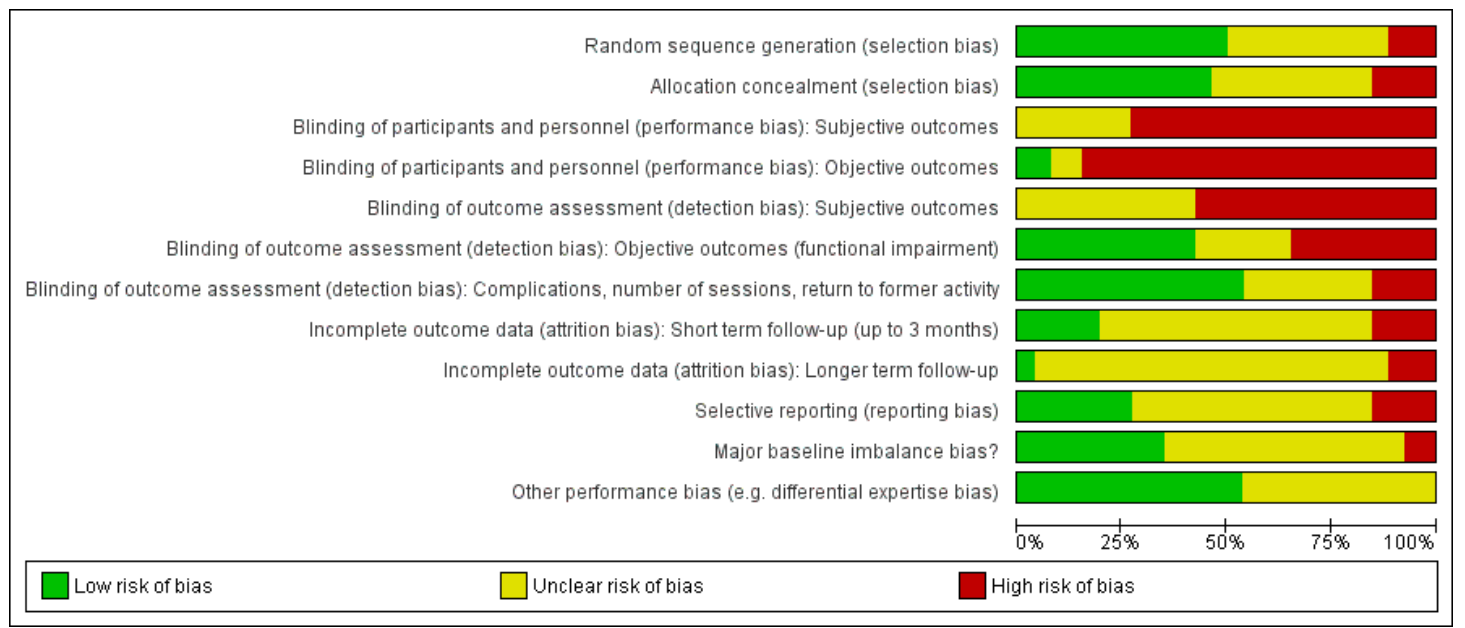

\begin{abstract}
Allocation
Eleven studies were at low risk of selection bias reflecting both random sequence generation and allocation concealment (Brehmer 2014; Challis 2007; Cooper 2001; Gronlund 1990; Jongs 2012; Kay 2000; Kay 2008; Maciel 2005; Magnus 2013; Wakefield 2000; Watt 2000). Four studies were at high risk of selection bias, three of which were quasi-randomised with allocation based on dates of birth (Basso 1998) or alternation (Lazovic 2012; Rozencwaig 1996); and allocation concealment was considered unlikely in Taylor 1994, where a coin was tossed. Of the remainder, which were judged at unclear risk of bias, no details of the method of randomisation was provided in four trials (Bighea 2013; Knygsand-Roenhoej 2011; Pasila 1974; Toomey 1986) and insufficient details relating either to sequence generation or safeguarding allocation concealment, or both, for seven trials (Bache 2001; Cheing 2005; Christensen 2001; Krischak 2009; Kuo 2013; Souer 2011; Svensson 1993).
\end{abstract}

\section{Blinding}

Blinding of participants and treatment providers was impractical in most of these studies, putting these at high risk of performance bias for both subjective outcomes, where reported, and objective outcomes. There were four exceptions, where the use of sham controls (pulsed electromagnetic field (PEMF), ultrasound) or blinding of independent care providers put two studies at low risk of performance bias for objective outcomes (Basso 1998; Magnus 2013) and two studies at unclear risk (Cheing 2005; Souer 2011). Though the use of sham PEMF treatment allowed participant blinding in Cheing 2005, participants were not blinded for the ice treatment aspect of this trial.

Fourteen of the 20 trials reporting subjective outcomes were at high risk of detection bias and the remaining six were at unclear risk of bias for these outcomes. Sixteen trials reported assessor blinding, most commonly for measures of functional impairment (e.g. grip strength), but the effectiveness of the blinding was in question for some of these. In all, 11 trials were at low risk of detection bias for functional impairment measures (Basso 1998; Challis 2007; Christensen 2001; Kay 2000; Kay 2008; Knygsand-Roenhoej 2011; Lazovic 2012; Maciel 2005; Magnus 2013; Toomey 1986; Watt 2000), nine were at high risk of detection bias (Bighea 2013; Brehmer 2014; Cooper 2001; Jongs 2012; Krischak 2009; Pasila 1974; Rozencwaig 1996; Svensson 1993; Taylor 1994), and six were at unclear risk of detection bias for this item. Fourteen of the 22 trials reporting on outcomes in the third category of outcomes (complications, number of sessions, return to former activity) were at low risk of detection bias for this item. Four of the other eight trials were at high risk of bias (Brehmer 2014; Rozencwaig 1996; Taylor 1994; Watt 2000) and four were at unclear risk of bias (Jongs 2012; Knygsand-Roenhoej 2011; Pasila 1974; Svensson 1993). 


\section{Incomplete outcome data}

The risk of attrition bias was assessed separately for short-term and long-term outcomes. Five trials reporting either no or very few losses were at low risk of attrition bias at short-term follow-up (Cooper 2001; Kay 2000; Kuo 2013; Souer 2011; Taylor 1994). Another four trials were at high risk of attrition bias at short-term follow-up; there was an imbalance between treatment groups in the loss to follow-up in two trials (Kay 2008; Magnus 2013) and large losses to follow-up in Pasila 1974 and Svensson 1993. The remaining 17 trials were at unclear risk of attrition bias, often reflecting uncertainly on the effect of the trial results from some small imbalances in losses to follow-up or post-randomisation exclusions, insufficient information on participant flow or inappropriately timed follow-up. Of the seven trials with long-term follow-up, one was at low risk of attrition bias (Knygsand-Roenhoej 2011), three trials with either incomplete data (Christensen 2001) or some losses that were reasonably balanced between the two groups (Maciel 2005; Souer 2011) were at unclear risk of attrition bias, and three were at high risk of attrition bias (Brehmer 2014; Magnus 2013; Wakefield 2000).

\section{Selective reporting}

Judgement of risk of reporting bias was hampered by the absence of published protocols and that trial registration documents were available for four studies only (Bache 2001; Jongs 2012; Kay 2008; Souer 2011). Seven studies were at low risk of reporting bias; this reflected a consistent and sufficiently comprehensive reporting of the trial results that gave no cause for concern (Brehmer 2014; Cooper 2001; Jongs 2012; Kay 2000; Kuo 2013; Souer 2011; Watt 2000). Four studies were at high risk of reporting bias; two of these were inadequately reported in brief (Bighea 2013; Rozencwaig 1996), one failed to report on some outcomes by treatment group (Pasila 1974) and there was some evidence of post hoc decisions for the fourth study (Wakefield 2000). The risk of reporting bias was unclear for the remaining 15 studies.

\section{Other potential sources of bias}

Nine trials provided sufficient information indicating comparability in key baseline characteristics, such as gender, age, fracture type and prior treatment and were at low risk of bias of confounding from major imbalances between trial groups in baseline characteristics. Two trials were at high risk of bias for this item: there were marked differences in gender ratio and age in Challis 2007, and a clinically significant difference in the mean ages of the two groups (60.75 versus 69.67 years) in Cooper 2001. The remaining 15 trials were at unclear risk of bias, often because of a lack of baseline data.

Comparability of care programmes, comprising interventions other than the trial interventions, is generally hard to confirm. However, it was considered likely in 14 trials, which were thus at low risk of performance bias. The other 12 trials were at unclear risk of bias. This was either because, as in six trials, there was insufficient information to confirm comparability (Bighea 2013; Kay 2008; Krischak 2009; Maciel 2005; Rozencwaig 1996; Souer 2011 ) or because, as in six trials, there were some actual or potential differences in care programmes that could have made some unknown, but perhaps small difference (Basso 1998; Cooper 2001; Gronlund 1990; Knygsand-Roenhoej 2011; Magnus 2013; Watt 2000).

\section{Effects of interventions}

The outcomes reported in the included studies trial reports are listed in the Characteristics of included studies tables. The results presented below are ordered by the comparisons given in the Included studies section.

We based our judgement of clinically important between-group mean differences in three patient-reported outcome measures using the following minimal clinically important differences (MCIDs); alternative sources and values are listed in Primary outcomes. (Note, MCIDs are absolute values and thus direction of effect is independent).

DASH: 10 (Sorensen 2013; this included people with non-surgical treatment for isolated tendonitis, arthritis, or nerve compression syndromes from the forearm to the hand).

QuickDASH: 14 (Sorensen 2013; as above).

PRWE: 11.5 (Walenkamp 2015; distal radius fractures).

\section{(I) Rehabilitation intervention versus no intervention}

\section{( Ia) Rehabilitation started during the definitive treatment period}

\section{Occupational or other hand therapy}

Two trials (Cooper 2001; Gronlund 1990) provided routine therapy during plaster cast immobilisation. Cooper 2001 evaluated "early therapeutic intervention" started within four days of injury and plaster cast application versus no intervention in 17 people. Gronlund 1990 compared the provision of "occupational therapy" one to three days after the application of a plaster cast to no provision in 40 participants. We considered pooling the data despite the differences between the interventions of these two trials. However, no data were available for pooling and so we have presented the results of the trials separately in the text below.

After plaster cast removal, one participant of the treatment group versus five participants in the control group of Cooper 2001 met the criteria for attendance of the hand therapy group classes (see Analysis 1.1: risk ratio (RR) $0.23,95 \%$ confidence interval (CI) 0.03 to 1.54 ). At four weeks post-immobilisation, Cooper 2001 reported no statistically significant differences between the two 
groups in the Disability of the Arm, Shoulder and Hand (DASH) questionnaire scores (median: 22.50 versus 45.00 (higher scores = greater disability); reported $\mathrm{P}=0.06$ ) or time to perform the nine hole peg test (median: 19.00 versus 27.00 seconds; reported $\mathrm{P}=0.12$ ). This contrasts with the results of statistically significant differences between the two groups at four days post-immobilisation (median DASH scores: 46.00 versus 61.00 , reported $\mathrm{P}=$ 0.02; median nine hole peg test time: 22.00 versus 48.00 seconds, reported $\mathrm{P}=0.02$ ). At four weeks post-immobilisation, the intervention group had statistically and clinically significantly better grip strength (see Analysis 1.2: mean difference (MD) $7.28 \mathrm{~kg}$, $95 \%$ CI 1.24 to $13.32 \mathrm{~kg}$ ), and range of motion (see Analysis 1.3, supination: MD 18.33 degrees, $95 \%$ CI 6.41 to 30.25 degrees; extension: MD 10.94 degrees, $95 \%$ CI 0.80 to 21.08 degrees; ulnar deviation: MD 15.03 degrees, $95 \%$ CI 9.78 to 20.28 degrees). The difference in oedema was not statistically significant (see Analysis 1.4). Though pain was less in the intervention group, the differences were not statistically significant (see Analysis 1.5, any pain at rest: $0 / 8$ versus $4 / 9$, RR $0.12,95 \%$ CI 0.01 to 1.99 ). Cooper 2001 reported there were no statistically significant differences between the two groups in the pain during activity (visual analogue scale (0 to $100 \mathrm{~mm}$ : higher scores = worse pain): median 25.50 versus 41.00 , reported $P=0.63$ ). Finger mobility was statistically significantly better in the early therapy group (see Analysis 1.6), perhaps reflecting the attention paid to finger exercises in this group. Three types of pinch grip were also reported to be statistically significantly better in the early therapy group (e.g. median 'tip pinch grip': 4.00 versus 2.25 ; reported $\mathrm{P}=0.04$ ). There were no cases of reflex sympathetic dystrophy.

Of the 17 participants in Gronlund 1990 assigned to occupational therapy, 16 were provided with appliances, such as angled knives, and 10 were given home help. Plaster cast fitting problems were found in four participants and were resolved by a subsequent visit to the casualty ward. Nine occupational therapy participants were found not to have understood the core instructions for exercises and information provided by an occupational therapist to all trial participants after their initial treatment. Following plaster cast removal at five weeks, the functional scores (Stewart 1984) of the 17 participants allocated to occupational therapy were reported as being statistically significantly better than those for the 23 participants in the group receiving no occupational therapy (median score 13 versus 18 ; reported $\mathrm{P}<0.05$ ). Stewart 1984 based their functional grading scheme on Gartland 1951 and graded 9 to 14 as "fair" and 15 and above as "poor". Wrist mobility also tended to be greater in the occupational therapy group (median percentage range of motion compared with unaffected wrist: $60 \%$ versus $50 \%$; reported $\mathrm{P}=$ non-significant). However at three months, both groups had similar hand function (median functional score: 10 versus 9; reported $\mathrm{P}=$ non-significant) and wrist mobility (median relative mobility: $80 \%$ versus $80 \%$ ). Similar numbers of participants in the two groups developed reflex sympathetic dystrophy (see Analysis 1.7: 3/17 versus 2/23; RR 2.03, 95\% CI 0.38 to 10.84). There were no cases of median or ulnar nerve compression or tendon rupture. All of the participants of the occupational therapy group who had been questioned expressed satisfaction with the intervention and indicated that they had not been inconvenienced. Control group participants were not asked about their understanding of the initial set of instructions, nor to rate satisfaction or convenience. The numbers in either group referred for occupational therapy after the plaster cast removal at five weeks were not recorded (Gronlund 2001).

\section{Cyclic pneumatic soft tissue compression}

Challis 2007 evaluated the use of cyclic pneumatic soft tissue compression using an inflatable cuff placed under the plaster cast in 21 patients, reporting results for 19 patients at six weeks (end of treatment) and 10 weeks post-fracture. Grip strength was significantly higher in the intervention group at both six weeks (MD $5.55 \mathrm{~kg}$, $95 \%$ CI $2.52 \mathrm{~kg}$ to $8.58 \mathrm{~kg}$ ) and 10 weeks (MD $10.40 \mathrm{~kg}, 95 \%$ CI $4.66 \mathrm{~kg}$ to $16.14 \mathrm{~kg}$ ) (see Analysis 2.1). Similar findings applied to pinch strength (see Analysis 2.2). The range of motion results also favoured the intervention group (flexion/extension range of motion at six weeks: MD $17.70^{\circ}, 95 \% \mathrm{CI}-0.05^{\circ}$ to $35.45^{\circ}$; flexion/extension range of motion at 10 weeks: MD $30.71^{\circ}, 95 \% \mathrm{CI}$ $2.61^{\circ}$ to $58.81^{\circ}$; supination/pronation at six weeks: MD $42.52^{\circ}$; $95 \%$ CI $5.96^{\circ}$ to $79.08^{\circ}$; and supination/pronation at 10 weeks: MD $19.71^{\circ} ; 95 \% \mathrm{CI}-19.13^{\circ}$ to $58.55^{\circ}$ ), although the results were not statistically significant for the first and last results reported here (see Analysis 2.3). Challis 2007 reported that participants in the intervention group delivered the compression $93 \%$ of the time during the immobilisation period. Additionally, participants of both groups performed their exercise programme $90 \%$ of the time in the four weeks after immobilisation.

\section{Early digit mobilisation during external fixation}

Kuo 2013 evaluated a progressive early digit mobilisation programme provided by an occupational therapist during six weeks of external fixation in 22 patients, reporting results for all participants at one, three, seven weeks (one week after end of intervention and external fixation) and 12 weeks post-fracture. Using a modified Taiwanese version of the MAM-36 (45 to 190: best outcome), Kuo 2013 found no significant difference between the two groups at three, seven or 12 weeks (12 weeks: MD 8.30 favouring digit mobilisation, 95\% CI -5.17 to 21.77 ; see Analysis 3.1). Grip, pinch and 'three jaw chuck' pinch strengths, expressed as percentage of those of the uninvolved hand were greater in the intervention group but none of the differences were statistically significant and the potential benefit at seven weeks was much reduced at 12 weeks follow-up (see Analysis 3.2). Range of digit motion, expressed as finger "workspace" and thumb "workspace", was greater in the intervention group (see Analysis 3.3) but the clinical importance of this is unclear. Assessment of finger dexterity via the 
Purdue pegboard test showed no significant differences between groups. Based on the measures of radial inclination, radial height and volar tilt (9.9 versus 9.8 degrees), no differences were found between the intervention and control groups in radiological outcome at 12 weeks.

\section{Pulsed electromagnetic field (PEMF)}

Lazovic 2012 compared 10 sessions of pulsed electromagnetic field therapy over two weeks versus no therapy control during cast immobilisation in 60 older women. Follow-up was two to three days after cast removal. The PEMF group had lower and thus better mean PRWE pain and activity/function scores but the 95\% CIs crossed the line of no effect and may not include a clinically important effect (PRWE pain: MD -2.87, 95\% CI -7.07 to 1.33; PRWE function: MD $-0.80,95 \%$ CI -5.29 to 3.69; see Analysis 4.1). Range of motion was better in the PEMF group with clinically borderline but statistically significant differences in supination, flexion and extension (see Analysis 4.2). Although there was evidence of less hand oedema in the PEMF group, the clinical relevance of the result is not clear (see Analysis 4.3). Fewer participants of the PEMF group had complications (2/30 versus 7/30; (RR 0.29, 95\% CI 0.06 to 1.26; see Analysis 4.4); however, the diagnosis of the two cases of CRPS type 1 was provisional. For all outcomes including complications, the inadequate length of follow-up means the consequences of these preliminary findings are unknown.

\section{Cross-education (strength training of the non-fractured hand)}

Magnus 2013 reported the results of 'cross-education' intervention of strength training of the non-fractured hand in 39 of the original 51 participants at 9,12 and 26 weeks. Ten of the 12 participants ( 7 in the cross-education group versus 3 in the control group) not included in the analyses had withdrawn because they were no longer interested in participating; one participant had unrelated health problems and one participant was excluded because of inadequate adherence to the cross-education programme. Magnus 2013 found no statistically significant differences between the cross-education and control groups in PRWE scores (0 to 150: worst outcome) at the three follow-up times ( 9 weeks: MD -11.00, 95\% CI -32.85 to 10.85 ; 12 weeks: MD $-9.80,95 \%$ CI -32.73 to 13.13 ; and 26 weeks: MD 4.20, 95\% CI -9.57 to 17.97 ; see Analysis 5.1). Statistically and clinically significant differences between the two groups in grip strength of the fractured hand results occurred only at 12 weeks, where there were better results in the cross-education group ( 9 weeks: MD $1.20 \mathrm{~kg}, 95 \% \mathrm{CI}-3.60$ to $6.00 \mathrm{~kg} ; 12$ weeks: MD $5.50 \mathrm{~kg}, 95 \%$ CI 1.28 to $9.72 \mathrm{~kg} ; 26$ weeks: MD $3.40 \mathrm{~kg}, 95 \%$ CI -0.83 to $7.63 \mathrm{~kg}$; see Analysis 5.2). A similar finding in favour of cross-education at 12 weeks but not at 9 or 26 weeks applied to combined supination-pronation range of motion (MD 14.30 degrees, 95\% CI 4.42 to 24.18 degrees; see Analysis 5.3) and combined flexion-extension range of motion (MD 20.30 degrees, 95\% CI 5.16 to 35.44 degrees; see Analysis 5.4). It is noteworthy that there were consistently more missing data points in the control group for all outcomes at 12 and 26 weeks; in contrast, more people allocated to cross-education withdrew.

\section{(Ib) Rehabilitation started post-immobilisation}

\section{Single session of physiotherapy, primarily advice and instructions for a home exercise programme versus no intervention}

Kay 2008 compared a single session of physiotherapy, primarily advice and instructions for a home exercise programme versus no intervention after cast removal in 56 people. Follow-up assessment was at three and six weeks follow-up. PRWE scores for pain and function were lower (better outcome) in the physiotherapy group at both three weeks (PRWE pain: MD -18.00, 95\% CI -31.20 to -4.80; PRWE function: MD -14.00, 95\% CI -28.82 to 0.82 ; 48 participants) and six weeks (PRWE pain: MD -14.00, 95\% CI 26.82 to -1.18 ; PRWE function: MD $-10.00,95 \%$ CI -23.30 to 3.30; 47 participants; see Analysis 6.1).

These results show a clinically important effect of the intervention for the intervention at three weeks: the mean differences were greater than the MCID of 11.5 estimated for the PRWE. Although the mean differences at six weeks were less than the MCID, both 95\% CIs include the MCID and thus the potential for a clinically important effect in favour of the intervention as well as a no clinically important difference between the two groups.

These results show a clinically important effect, although reduced after six weeks, of the intervention as all $95 \%$ CIs included the MCID of 11.5 estimated for the PRWE (Walenkamp 2015). The QuickDASH results, split by general, sports and work functioning, also favoured the physiotherapy intervention (see Analysis 6.2). Kay 2008 found no clinically important between-group differences at six weeks in grip strength (see Analysis 6.3) or range of motion (see Analysis 6.4 and Analysis 6.5). Complications (carpal tunnel syndrome, scar, instability) were detected at follow-up assessment in two physiotherapy group participants and one control group participant (see Analysis 6.6). There were slightly fewer requests for more physiotherapy in the physiotherapy group $(6 / 28$ versus 10/28; RR 0.60, 95\% CI 0.25 to 1.43 ; see Analysis 6.7).

\section{Physiotherapy or occupational therapy}

Routine provision of therapy after plaster cast removal was compared with no provision in four trials (Bache 2001; Christensen 2001; Maciel 2005; Wakefield 2000). All participants in each of these trials received instructions for home exercises from either an occupational therapist (Christensen 2001) or a physiotherapist 
(Bache 2001; Maciel 2005; Wakefield 2000). We considered pooling despite the differences between the interventions of the four trials. However, no data were available for pooling and we have separately presented the results of the four trials in the text below. No statistically significant differences were reported between the two groups in functional scores (Solgaard 1988 based on Gartland 1951 ) in Christensen 2001, at either three (median score: 8 versus 6 ) or nine months (median score: 3 versus 2). (In this functional grading scheme, Solgaard rated a grade of 0 to 2 as "excellent", 3 to 7 as "good" and 8 to 18 as "fair".) Grip strength was also similar in the two groups (see Analysis 7.3) at the two follow-up times. Participants allocated occupational therapy attended an average of 37.5 therapy sessions (range 22 to 90 sessions), of overall duration averaging 11.4 hours (range 6 to 22 hours). No participants in the control group received occupational therapy.

Bache 2001 found that while the baseline patient characteristics of the two groups were generally comparable, the participants allocated physiotherapy were more "symptomatic", with significantly reduced wrist extension (median: 15 versus 25 degrees; reported $\mathrm{P}$ $=0.03)$, and tendencies to poorer pronation $(\mathrm{P}=0.05)$, supination $(\mathrm{P}=0.06)$ and ulnar deviation $(\mathrm{P}=0.08)$. (Adjustments were made for multiple testing throughout the analysis of this trial). The trialists considered that the outcome in both groups at 12 weeks follow-up was acceptable with no statistically significant differences between the two groups found for any of the six range of movement measures, the functional status scores (Levine 1993), pain scores or grip strength. This suggests a trend to a greater improvement over time from a more unfavourable starting position in the physiotherapy group; as reported by Bache 2001. In the light of the differences observed at baseline, the findings of an "Area under the curve" analysis, which included the results from the baseline, at four weeks, and where available, at 12 weeks for 81 of the 98 participants, were presented. There were no statistically significant differences in the outcome measures aside from supination which was significantly better in the control group (adjusted $\mathrm{P}=0.04$ ); this reflected the better baseline scores for this outcome measure in the control group, which persisted throughout follow-up. Similar numbers of participants in the two groups developed complications: reflex sympathetic dystrophy (two versus three) and carpal tunnel syndrome (two versus two). Five of these participants (four with CRPS-1; one with carpal tunnel syndrome) were excluded from 12-weeks follow up; and complications (one CRPS-1; one carpal tunnel syndrome) developed in two physiotherapy group participants at the end of the study. The median duration of treatment for participants allocated physiotherapy was 35 days (range 1 to 142 days) and the median number of contacts was three (range 1 to 16). Four physiotherapy participants were referred to occupational therapy. None of the control group participants retained in the trial received physiotherapy or occupational therapy, aside from the advice and instructions given initially to all trial participants.

Wakefield 2000 similarly found no statistically significant differ- ences between the two groups in overall function (Sheehan 1983) (presented as the degree of difficulty in carrying out activities of daily living relative to the unaffected side), relative grip strength, or pain, at three or six-months follow-up (see Analysis 7.2; Analysis 7.4 and Analysis 7.5). Of the measures for range of motion, the only statistically significant difference between the two groups was in wrist flexion and extension relative to the unaffected side at six months (MD 12.20\%, 95\% CI 5.41 to $18.99 \%$ ); see Analysis 7.7 and Analysis 7.8. Functional assessment at six months was limited to 66 participants, compared with 90 at three months. No significant differences between the two groups were reported in any of the measures of quality of life at six months, as assessed from questionnaire data from 50 participants. Participants allocated physiotherapy attended a median of three sessions (range 1 to 22 sessions). Two participants in the control (no physiotherapy) group were referred for physiotherapy after the three-month assessment due to problems with returning to full function.

Maciel 2005 found no statistically significant differences between up to six weeks of "activity-focussed" physiotherapy compared with one advice session (control group) in terms of overall function, or in terms of pain, activity or disability as rated by the PRWE score (MacDermid 2000) at 24 weeks: see Analysis 7.1. They also found no statistically significant differences between the two groups in grip strength, and in wrist extension and flexion results (see Analysis 7.3 and Analysis 7.6). These results, however, applied to just 33 (73\%) of the 45 people originally recruited into the trial. Of these 45 , baseline measurements were not available for four people. A further four people in each group "withdrew" by 24 weeks follow-up. Of the four participants in the physiotherapy group, two failed to attend, one was "too busy", and the fourth sought a second orthopaedic opinion. For the control group, two failed to attend, one died and one required a "general anaesthetic manipulation procedure”. Maciel 2005 reported no adverse events related to the interventions. The mean number of treatment sessions in the physiotherapy group was 4.4 compared with 0.9 in the control group (see Analysis 7.9).

\section{Continuous passive motion}

Very limited information and results are available for Rozencwaig 1996; a very small trial of seven participants who had been treated with external fixation. The three participants given continuous passive motion therapy on top of the usual occupational therapy took less time to achieve a completely independent status than the four control (no continuous passive motion) group participants (see Analysis 8.1: MD -1.80 weeks, 95\% CI -3.24 to -0.36 weeks). Rozencwaig 1996 reported that the recovery of range of motion of the affected wrist was also quicker in participants receiving continuous passive motion.

\section{Pulsed electromagnetic field (PEMF)}


The final outcome assessment for Cheing 2005 preceded the last treatment session on the fifth day. The results for pain, oedema (volume) and range of motion are presented in Analysis 9.1 and Analysis 9.2. In these, the results for two intervention groups (PEMF plus ice; PEMF) were combined for the PEMF group, and the results of the two intervention groups (sham PEMF plus ice; sham PEMF) were combined for the sham group. None of the differences between the combined PEMF groups and combined sham PEMF groups were statistically significant. There were no adverse effects recorded.

\section{Ice}

As above, the final outcome assessment for Cheing 2005 preceded the last treatment session on the fifth day. The results for pain, oedema (volume) and range of motion are presented in Analysis 10.1 and Analysis 10.2. In these, the results for two intervention groups (PEMF plus ice; sham PEMF plus ice) were combined for the ice group, and the results of the two intervention groups (PEMF; sham PEMF) were combined for the control group. Pain was statistically significantly less in the combined ice groups (visual analogue scale: $\mathrm{MD}-0.82 \mathrm{~cm}, 95 \%-1.33$ to $-0.31 \mathrm{~cm})$. In contrast, extension was significantly better in the control groups (MD -8.89 degrees, $95 \%$ CI -13.57 to -4.21 degrees). This, however, should be seen in the context of the significantly higher baseline extension mean value for the control groups: this was 8.44 degrees greater than that of the combined ice groups. Differences between the two groups in the other outcome measures were not statistically significant. There were no adverse effects recorded.

\section{Pulsed electromagnetic field (PEMF) plus ice}

The combined intervention was compared with sham PEMF alone in 39 participants of Cheing 2005. The results for pain, oedema (volume) and range of motion at the final assessment on the fifth day are presented in Analysis 11.1 and Analysis 11.2. Only the differences between the two groups in extension in favour of the control group (MD -9.20 degrees, 95\% CI -16.79 to -1.61 degrees), and ulnar deviation in favour of the combined intervention group (MD 3.80 degrees, $95 \%$ CI 0.65 to 6.95 degrees) were statistically significant. Again, the more favourable result in the control group for extension may reflect the significantly higher baseline extension mean value for this group (this was 11.6 degrees higher than that of the combined intervention group). There were no adverse effects recorded.

\section{Passive mobilisation}

Though the format and context of the passive mobilisation differed considerably in the two trials investigating this modality (Kay 2000; Taylor 1994), there are sufficient similarities, including the declared experience of the physiotherapists involved, in the two trials to consider pooling. In the event, this was only possible for one outcome (number of treatments). However, pooling revealed highly statistically significant heterogeneity. We decided in this update not to pool these results but to present the results of the two trials separately (see Analysis 12.5).

Results of Kay 2000 were unavailable for one person, who withdrew because he found passive mobilisation too uncomfortable. Of the 39 participants remaining, no statistically significant differences were found between the two groups at six weeks for grip strength (see Analysis 12.1), range of motion (see Analysis 12.2), web space angle (see Analysis 12.3), finger movements (flexor deficit: reported P > 0.25; extensor deficit: reported $\mathrm{P}>0.39$ ) or visual analogue pain scores ( 0 : no pain to 10 : worst imaginable; median scores extracted from graph 1.25 versus 1.0 ; reported $\mathrm{P}$ $=0.63)$. Likewise no statistically significant differences between the groups were reported for subjective disability: visual analogue scores (0: no difficulty to 10: extreme difficulty; median scores extracted from graph: 2 versus 2 ; reported $\mathrm{P}=0.43$ ); or in the performance of six functional tests (reported $\mathrm{P}>0.18$ ); most participants were able to perform the latter without difficulty at six weeks. The four participants in the passive mobilisation group with complications present at six weeks had been treated conservatively: two had carpal tunnel syndrome; one had complex regional pain syndrome, ongoing from the start of the trial; and one participant had a malunited fracture. One osteoporotic participant in the control group who had received pins and plaster had unresolved finger stiffness at six weeks. Overall, there was no statistically significant difference in the numbers of participants with complications at six weeks (4/19 versus $1 / 20$; RR 4.21, 95\% CI 0.52 to 34.37 ; P $=0.18$; analysis not shown). Participants allocated passive mobilisation received on average six more treatments than those in the control group (MD 5.90, 95\% CI 5.40 to 6.40; see Analysis 12.5). Kay 2000 calculated that the mean total of hospital reimbursement, based on 1997 to 1998 costs, was nearly three times greater for the passive mobilisation group (AUD 457 versus AUD 161). In Taylor 1994, discharge from physiotherapy, at an average of 26 days, was at the discretion of physiotherapists, who based their decision on an acceptable range of motion or an assessment that no further benefit from therapy was to be expected. Participants receiving passive mobilisation tended to have slightly more treatment and took longer to be considered ready for discharge, but neither result was statistically significant (see Analysis 12.5 and Analysis 12.6). There was no statistically or clinically significant difference between participants receiving passive mobilisation and those receiving soft tissue massage (control group) in wrist extension at end of therapy (see Analysis 12.4: MD -2.14 degrees, 95\% CI -10.44 to 6.16 degrees). Taylor 1994 reported that subgroup analyses looking at wrist extensions attained by both groups of participants treated by three out of the four therapists involved showed no significant differences. They suggested that this finding showed that no one therapist was more proficient at applying passive joint mobilisation. 


\section{Intermittent pneumatic compression}

Data for three participants, excluded due to CRPS-1, psychiatric hospitalisation and death, were not provided in Svensson 1993. It is also likely that nine of the remaining 40 participants were unavailable for outcome assessment at three months. Svensson 1993 reported that grip strength and the various measures of movement tended to be better in the group given intermittent pneumatic compression at the start of each session of occupational therapy. However, only the results for wrist extension were statistically significantly better in the compression group (median 58 degrees versus 45 degrees; reported $\mathrm{P}<0.05$ ). A similarly non-statistically significant tendency for less pain at rest and during function was reported for the compression group. No reduction in oedema could be demonstrated for either group of participants. Only a few participants in each group (numbers not stated) were considered to require further occupational therapy after three weeks.

\section{Ultrasound}

Basso 1998 found no significant difference between participants allocated active ultrasound and those allocated sham ultrasound (control) in the loss of active flexion-extension wrist motion relative to the unaffected wrist (median loss: $15 \%$ versus $15 \%$ ); see Analysis 13.1. Based on persistent radiocarpal pain and delayed recovery of hand function, fewer ultrasound participants were referred for physiotherapy at eight weeks (see Analysis 13.2: 2/19 versus $8 / 19$; RR 0.25 , 95\% CI 0.06 to 1.03 ). There was no indication whether any participants received physiotherapy before eight weeks.

\section{Whirlpool}

It was not clear whether any participants were excluded from Toomey 1986 because of lack of improvement or deterioration in their condition. Also unclear is how many participants stopped treatment before the scheduled 12 sessions, and whether early curtailment was instigated by the therapist or the patient. By the end of treatment, at a maximum of six weeks, there were no statistically significant differences between those participants whose affected forearm was immersed in a whirlpool or wrapped in two towels (control group) in grip strength, pain or forearm and wrist range of motion (see Analysis 14.1, Analysis 14.2 and Analysis 14.3). Although, as seen in Analysis 14.4, finger flexion tended to be worse in the whirlpool group, and statistically significantly worse for flexion of the long finger (MD -7.50 degrees, 95\% CI -13.52 to -1.48 degrees), Toomey 1986 questioned the clinical significance of these results. Follow-up immediately after the session (whirlpool or towel) revealed a statistically significantly higher oedema in the whirlpool group (see Analysis 14.5: MD $72.92 \mathrm{~mL}$, 95\% CI 5.89 to $139.95 \mathrm{~mL}$ ), without statistically significant differences in strength, pain, or forearm and wrist range of motion. Long-term oedema was not statistically significant between the two groups (see Analysis 14.5). Participants were reported as finding the whirlpool comfortable and pleasant; no comments from the towel group were reported. Toomey 1986 referred to whirlpool baths as being an "expensive modality" but did not quantify costs.

\section{Dynamic wrist extension splint}

Jongs 2012 provided individual patient data and tables which enabled the presentation of intention-to-treat analyses. The results, however, did not differ importantly from the per-protocol analyses reported in the main report. Jongs 2012 found no statistically or clinically significant differences between the dynamic splint and control group in the PRWE results at the end of the eight weeks treatment (MD - $0.87 \%$, 95\% CI -7.43\% to 5.59\%) nor one month subsequently (MD 3.57\%, 95\% CI $-3.18 \%$ to $10.32 \%$; see Analysis 15.1). Similar findings applied at 12 weeks to the results of the Canadian Occupational Performance Measure (see Analysis 15.2) and range of motion (see Analysis 15.3). There were two adverse events reported in relation to the use of dynamic splints: transient numbness in index finger and pain during stretch. Both resolved when the splints were modified. Repairs were required for two other splints. All four participants were without their splints for between one to 13 days. Fourteen participants provided data on their splint use; this showed that most participants did not wear their splints for the recommended six hours a day and that there were days when participants did not wear their splint at all. While adherence to exercises was poorly recorded in the participants' personal diaries, Jongs 2012 reported that there was no indication of a systematic difference between the two groups.

\section{2) One rehabilitation intervention versus another rehabilitation intervention}

\section{(2a) Rehabilitation started during the definitive treatment period}

No trials were identified.

\section{(2b) Rehabilitation started post-immobilisation}

\section{Physiotherapy versus instructions for home exercises by an orthopaedic surgeon}

The results for one uncooperative participant in the physiotherapy group and one participant referred to physiotherapy by their general practitioner in the control (instructions from an orthopaedic surgeon) group were excluded from the analyses of Watt 2000. At an average of six-weeks follow up, the median grip strength of the physiotherapy group participants was reported to be significantly greater $(10.0 \mathrm{~kg}$ versus $5.3 \mathrm{~kg})$. Wrist extension was also found to be significantly better in the physiotherapy group (see Analysis 
16.1: MD 17.40 degrees, 95\% CI 6.49 to 28.31 degrees). Physiotherapy group participants attended an average of five sessions.

\section{Pulsed electromagnetic field (PEMF) versus ice}

The final outcome assessment for Cheing 2005 which compared these two interventions preceded the last treatment session on the fifth day. The results for pain, oedema (volume) and range of motion at the final assessment are presented in Analysis 17.1 and Analysis 17.2. Only the differences between the two groups in pain, which favoured ice (visual analogue scale: MD $1.10 \mathrm{~cm}, 95 \%$ CI 0.48 to $1.72 \mathrm{~cm}$ ), and extension, which favoured PEMF (MD 8.40 degrees, $95 \%$ CI 2.32 to 14.48 degrees) were statistically significant. Notably, these are consistent with similar differences in baseline values: the mean initial pain was significantly greater in the PEMF group $(4.3 \mathrm{~cm}$ versus $3.4 \mathrm{~cm}$ ); but the difference in baseline extension (33.9 degrees versus 28.4 degrees) between the two groups was not statistically significant. There were no adverse effects recorded.

\section{Modified "manual edema mobilization" (MEM) versus 'traditional' oedema treatment}

Knygsand-Roenhoej 2011 excluded one participant who did not comply with MEM treatment, leaving in their analysis 29 patients who were being treated for subacute oedema related to distal radius fracture. Knygsand-Roenhoej 2011 reported, without presenting data, no statistically significant differences between the two groups in the score summing the performance of four bilateral activities of daily living at six weeks (reported $\mathrm{P}=0.49$ ) or nine weeks (reported $\mathrm{P}=0.34$ ) after the start of treatment. Similar findings of a lack of difference applied at nine weeks for the numbers of participants who had a clinically important improvement in the performance and satisfaction domains of the Canadian Occupational Performance Measure (see Analysis 18.1) and at six and 26 weeks for pain at rest and pain when active (see Analysis 18.2). Two complications, both reported in the discussion section of the article, occurred in the MEM group (2/14 versus 0/15; RR 5.33, 95\% CI 0.28 to 102.26; see Analysis 18.3). Oedema decreased in both groups over time but was always less, including at baseline, in the MEM group. The difference between the groups was statistically significant at nine weeks (MD $-16.20 \mathrm{ml} ; 95 \% \mathrm{CI}-31.30 \mathrm{ml}$ to $-1.10 \mathrm{ml}$; see Analysis 18.6), but borderline at six months (MD $13.20 \mathrm{ml}, 95 \% \mathrm{CI}-30.84 \mathrm{ml}$ to $4.44 \mathrm{ml}$; see Analysis 18.6). Fewer participants in the MEM group were receiving oedema treatment after six or nine weeks (see Analysis 18.5) and, overall, the MEM group received fewer occupational therapy sessions (MD -3.80; 95\% CI -8.48 to 0.88 ; see Analysis 18.4).

(3) Any method (context) of delivering or providing rehabilitation interventions versus any other method of delivering or providing rehabilitation interventions (3a) Rehabilitation started during the definitive treatment period

Exercise therapy supervised by a physiotherapist versus instructions for the same exercises given by an orthopaedic surgeon

At 12 weeks follow-up, Pasila 1974 found no significant differences in strength or range of motion between supervised participants and those given instructions by a surgeon after initial treatment (see Analysis 19.1 and Analysis 19.2: all data extracted from graphs in the trial report). (The relatively low mean values for radial deviation were not explained.) The results of 39 participants who had dropped out of the study were excluded from the analyses. Pasila 1974 reported that the 96 remaining participants returned to work approximately seven weeks after their injury, there being no statistically significant difference between the two groups for this outcome. Whilst over half of the participants (48/92) were reported as having a "positive attitude", at 12 weeks there was no indication if this differed between the two groups. The physiotherapy group participants visited the physical medicine department an average of four times (range 1 to 12 times) before they were able, in the therapist's opinion, to continue training on their own.

\section{(3b) Rehabilitation started post-immobilisation}

Physiotherapy versus a home exercise programme after cast immobilisation

Very limited results were available from Bighea 2013, which compared physiotherapy, primarily exercises, versus a home exercise programme in 20 participants who had had cast immobilisation. After four weeks of rehabilitation, Bighea 2013 reported better PRWE scores (lower scores indicate better hand function) for the 10 participants of the physiotherapy group ( 47.3 versus 54.7 ). Bighea 2013 also reported greater increases in range of motion in the physiotherapy group after four weeks: extension increased $71 \%$ versus $62.7 \%$; and flexion increased $63.2 \%$ versus $53.8 \%$ ).

\section{Physiotherapy or occupational therapy versus progressive} home exercise programme after volar plate fixation

Although the participants and comparisons of the two trials in this category were sufficiently similar to consider pooling, this did not apply to their timing of follow-up assessment and thus they are reported separately below.

At six weeks follow-up, Krischak 2009 found physiotherapy resulted in significantly poorer function as assessed using the PRWE compared with a home exercise programme (MD 17.60, 95\% CI 8.97 to 26.23; see Analysis 20.1). Krischak 2009 also found the physiotherapy group had significantly lower grip strength $(32 \%$ 
versus $54 \%$ of uninjured hand; reported $\mathrm{P}=0.003$ ), extension-flexion range of motion $(52 \%$ versus $79 \%$ of uninjured hand; reported $\mathrm{P}=0.001)$, ulnar-radial deviation range of motion $(59 \%$ versus $70 \%$ of uninjured hand; reported $\mathrm{P}=0.013$ ) but not in pronation and supination (data not reported). Two participants, one in each group, were excluded because of having additional physiotherapy sessions but otherwise, all other physiotherapy group participants had the prescribed 12 sessions. Returned exercise logs from 19 of the 23 participants in the home exercises group showed $97 \%$ exercises were documented as "done". Krischak 2009 reported that the cost of a 20- to 30-minute physiotherapist session was 18 Euros. Souer 2011 found no statistically or clinically significant difference between the two groups in their DASH scores at three or six months follow-up (3 months: MD 0.20, 95\% CI -4.28 to 4.68 ; 6 months: MD $-1.10,95 \%$ CI -4.36 to 2.16; see Analysis 20.2). Although Mayo wrist scores tended to favour the home exercises group, the difference between the two groups did not achieve statistical significance at the two follow-up times (e.g. 6 months: MD $-4.40,95 \%$ CI -9.51 to 0.71 ; see Analysis 20.3). There was no difference between the two groups in 'pain at rest' results at both follow-up times (see Analysis 20.4). The occupational therapy group had lower grip strength at both follow-up times but the difference between the two groups reached statistical significance at three months only (3 months: MD $-4.80 \mathrm{~kg}, 95 \% \mathrm{CI}-8.53 \mathrm{~kg}$ to $-1.07 \mathrm{~kg}$; 6 months: MD $-2.70 \mathrm{~kg}$; $95 \%$ CI $-6.39 \mathrm{~kg}$ to $0.99 \mathrm{~kg}$ ) see Analysis 20.5). The inconclusive results for pinch strength are shown in Analysis 20.6. While favouring the home exercises group, none of the range of motion results were statistically significant at three months (see Analysis 20.7). However, the occupational therapy group had marginally poorer wrist mobility at six months in terms of extension ( $\mathrm{MD}-7.00^{\circ}$; $95 \% \mathrm{CI}-12.41^{\circ}$ to $-1.59^{\circ}$ ), flexion, supination and ulnar deviation (see Analysis 20.8). The few complications, presented Analysis 20.9, seem unlikely to be a direct result of the interventions. One participant allocated home exercises requested an additional appointment for extra coaching.

\section{Accelerated versus usual rehabilitation after volar plate fixation}

One trial (Brehmer 2014) with 81 participants investigated accelerated (started at two weeks) versus usual rehabilitation (started at six weeks) after volar plate fixation. There was a lot of variation in the numbers available at the different follow-ups. Although DASH scores favoured accelerated rehabilitation at eight and 12 weeks and six months, the effect size dropped over time and the confidence intervals did not include the MCID of 10 in the two later follow-up times ( 8 weeks: MD -8.00, 95\% CI -12.55 to 3.45; 12 weeks: MD $-3.00,95 \%$ CI -5.81 to -0.19 ; 6 months: MD -2.00, 95\% CI -4.96 to 0.96; see Analysis 21.1). Grip strength at three and six months was also greater in the accelerated group compared the usual group, with a significant difference showing at six months (MD $12.00 \mathrm{lb}, 95 \%$ CI $2.16 \mathrm{lb}$ to $21.84 \mathrm{lb}$; see
Analysis 21.2). The inconclusive results for pinch strength at three and six months are shown in Analysis 21.3). While the range of motion results favoured the home exercises group at both three and six months, the only statistically significant between-group differences were for flexion. However, the difference was less and of uncertain clinical importance at six months ((MD 5.00 degrees, 95\% CI 0.10 to 9.90 degrees; see Analysis 21.5). The few complications, presented in Analysis 21.6, seem unlikely to be a direct result of the interventions. Notably, there was no loss of alignment.

\section{DISCUSSION}

\section{Summary of main results}

We set out to determine the effectiveness of rehabilitation interventions for adults with conservatively or surgically treated distal radial fractures. This encompassed the four basic questions stated in the 'Background': essentially, what interventions should be provided, by whom, when and for how long, and why? The variation in interventions, providers, timing, definitive treatment and patient characteristics makes this a complex and extensive area to review. We restricted the evidence to that from RCTs or quasiRCTs since these are generally less susceptible to systematic bias, specifically selection bias, than other study designs. Inevitably this has reduced the quantity of available evidence with only 26 small trials involving 1269 participants included so far. A further limitation is that only four of the 23 comparisons covered by these 26 trials were evaluated by more than one trial. Despite clearly different characteristics of trials testing essentially the same comparisons, pooling of trial results was nevertheless considered but, even in the very few cases where there were comparable outcomes, was not done.

The results for each of the 23 comparisons are summarised in five tables that present the evidence available for the following categories (Tables 5 to 9). The entry for each comparison in the tables presents the question asked (PICO), a summary of the results (Findings), an assessment of the quality of the evidence with reasons for downgrading (GRADE), and a note on the assessment of applicability based on five questions (see Table 4) together with other observations (Comments).

- Table 5 Rehabilitation intervention versus no intervention; rehabilitation started during the definitive treatment period: four comparisons, featuring five trials.

- Table 6 Rehabilitation intervention versus no intervention; rehabilitation started post-immobilisation: 11 comparisons featuring 13 trials (one trial tested three interventions: Cheing 2005). 
- No table. One rehabilitation intervention versus another rehabilitation intervention; rehabilitation started during the definitive treatment period: no trials.

- Table 7 One rehabilitation intervention versus another rehabilitation intervention; rehabilitation started postimmobilisation: three comparisons featuring three trials.

- Table 8 Any method (context) of delivering or providing rehabilitation interventions versus any other method of delivering or providing rehabilitation interventions; rehabilitation started during the definitive treatment period: one comparison featuring one trial.

- Table 9 Any method (context) of delivering or providing rehabilitation interventions versus any other method of delivering or providing rehabilitation interventions; rehabilitation started post-immobilisation: three comparisons featuring four trials.

It is noteworthy that with two exceptions, which are rated as low quality, the evidence for all comparisons has been rated as very low quality; both ratings indicate considerable uncertainty in the findings. Such ratings are almost inevitable where the evidence is derived from small, often methodologically flawed trials conducted in single centres with one or very few clinicians providing the intervention(s) under test.

It is instructive to consider the evidence staged by timing of the rehabilitation intervention rather than stick rigidly to the six comparisons above. As well as reflecting clinical context, this approach is taken because the structure of the review, which reflects the best categorisation of the often complex questions addressed in the individual trials, can lose sight of the other questions being addressed, in particular relating to the provision of these usually complex interventions. This restructuring, however, does not give rise to any new opportunities for pooling. The numbers of participants given below relate to the numbers for whom results were available.

\section{Rehabilitation during the definitive treatment period}

The evidence available for rehabilitation starting during the definitive treatment period is summarised in two tables (Table 5; Table 8 ); there being no trials comparing different rehabilitation interventions.

Two comparisons tested the provision of therapy provided by a rehabilitation specialist versus instructions only, in one comparison instructions were given by an orthopaedic surgeon, during cast immobilisation. One trial (17 participants) of the two trials in the first comparison provided very low quality evidence of a better hand function after hand therapy four days after plaster cast removal, with some beneficial effects continuing one month later (Cooper 2001). The other trial (40 participants) of this comparison found very low quality evidence of improved hand function in the short-term, but not in the longer-term (three months), for early occupational therapy (Gronlund 1990). The second comparison of physiotherapist-supervised exercise therapy versus instruction for the same exercises by an orthopaedic surgeon alone provided very low quality evidence of there being no difference in outcome between the two approaches (95 participants; Pasila 1980).

Four trials separately provided very low quality evidence of potential but limited benefits restricted to one or two measures of impairment of single interventions applied in addition to standard care (see Table 5). These aimed to counter well-known problems, such as finger stiffness and loss of grip strength, of cast immobilisation and to improve recovery. Two were provided in the clinic (early digit mobilisation during external fixation (22 participants; Kuo 2013); and pulsed electromagnetic field (PEMF) during cast immobilisation (60 participants; Lazovic 2012)) and two were applied at home after instructions (cyclic pneumatic soft tissue compression using an inflatable cuff placed under the plaster cast (19 participants; Challis 2007); cross-education during cast immobilisation with or without surgical repair (39 participants; Magnus 2013)). The very low quality and thus unreliable evidence on measures of functional impairment (e.g. grip strength) was available from the four small trials testing these interventions that, even where suggestive of potential yet limited benefit, should not be used to support their use in practice without further research.

\section{Rehabilitation started post-immobilisation}

The majority of the evidence for rehabilitation applied to the postimmobilisation period. This is summarised in three tables (Table 6; Table 7; Table 9); and includes three comparisons of different rehabilitation interventions (see Table 7). As above, the comparisons can be split into those relating to the provision of therapy and those of single interventions.

Seven comparisons tested the provision or format of therapy after post-immobilisation. The composition and extent of rehabilitation therapy provided by a rehabilitation specialist (usually physiotherapist or occupational therapist) varied considerably. The least of the interventions was a single session of physiotherapy, primarily advice and instructions for a home exercise programme, compared with 'no intervention' after cast removal (see Table 6). One trial (47 participants) found very low quality evidence in favour of the single session of physiotherapy (Kay 2008). The intervention group of Kay 2008 is similar to the 'control' group of the four heterogenous trials $(75,30,33$ and 66 participants) in the comparison of the routine provision of physiotherapy or occupational therapy in addition to instructions for home exercises versus instructions for home exercises from a therapist (Bache 2001; Christensen 2001; Maciel 2005; Wakefield 2000). As summarised in Table 6, none of the trials found a clinically significant effect of the routine provision of rehabilitation therapy post-immobilisation, but the available evidence is low quality. The limited and 
very low quality evidence from Watt 2000 (16 participants), which compared routine physiotherapy (average five sessions) versus instructions for home exercises by an orthopaedic surgeon after cast immobilisation, was in favour of physiotherapy (Table 7), as was the very low quality evidence from the comparison of physiotherapy versus a home exercise programme following cast immobilisation tested by Bighea 2013 (20 participants; see Table 9). Both trials (46 and 76 participants; Krischak 2009; Souer 2011) comparing physiotherapy or occupational therapy versus a progressive home exercise programme after volar plate fixation provided low quality evidence in favour of a structured programme of home exercises preceded by instructions or coaching (see Table 9). The last comparison of this group evaluated accelerated versus usual rehabilitation after volar plate fixation. There was very low quality evidence (63 participants; Brehmer 2014) of some potentially early benefit in DASH scores with accelerated rehabilitation that did not persist.

The other 11 comparisons were of specific interventions in addition to standard care (Table 6; Table 7). Six trials provided very low quality evidence on interventions that were applied following cast immobilisation, that had sometimes incorporated wires or pins: PEMF (83 participants; Cheing 2005), ice (83 participants; Cheing 2005), PEMF plus ice (39 participants; Cheing 2005), PEMF versus ice ( 44 participants; Cheing 2005), passive mobilisation (69 participants; Kay 2000 and Taylor 1994), intermittent pneumatic compression (31 participants; Svensson 1993), ultrasound (38 participants; Basso 1998) and immersion of the forearm in a whirlpool (24 participants; Toomey 1986). One trial provided very low quality evidence on continuous passive motion applied post-external fixation (7 participants; Rozencwaig 1996), and two trials provided very low quality evidence on interventions that were applied after either cast immobilisation or surgery: dynamic extension splint (40 participants; Jongs 2012), and manual oedema mobilisation versus traditional oedema treatment (29 participants; Knygsand-Roenhoej 2011). These trials provided very low quality evidence of a lack of clinically significant differences in outcome in patients receiving passive mobilisation, ice or PEMF or both these interventions, whirlpool immersion, or dynamic extension splint for patients with wrist contracture, compared with no intervention. This finding applied also to the two trials comparing PEMF versus ice and manual oedema mobilisation versus traditional oedema treatment. There was very low quality evidence of a short-term benefit of continuous passive motion post-external fixation, intermittent pneumatic compression and ultrasound therapy.

\section{Comment}

Before dismissing the evidence that is available as uninformative and unable to inform practice, or proposing further research, the questions asked and their context need also to be considered. Thus, the findings of the 23 comparisons are discussed under applicabil- ity in the next section.

\section{Overall completeness and applicability of evidence}

\section{Completeness of the evidence}

The evidence available for this review of 26 small trials is very limited, primarily because the evidence for 19 comparisons is derived solely from individual trials and that no pooling was possible for the four comparisons tested by up to a maximum of four trials. Further inadequacies arise from the limitations in outcome measurement, including the frequent lack of validated patient-reported outcome measures of wrist function, short follow-up periods, and incomplete reporting of results for some trials.

\section{General applicability of evidence}

\section{Results of the formal assessment of applicability}

As well as providing comprehensive details of the study populations and interventions in the Characteristics of included studies table, Table 4 shows our assessments for each trial of five aspects of relevance to ascertaining external validity: definition of the study population; description of the interventions; definition of primary outcome measures; relevance of outcome measurement and length of follow-up. Clearly unhelpful are incomplete descriptions of study inclusion (eight trials, of which one gave a very inadequate description (Bighea 2013)) and interventions (12 trials of which four gave a very inadequate description of the intervention (Krischak 2009; Rozencwaig 1996; Souer 2011; Wakefield 2000)). However, the trials that did not give full descriptions of their interventions were generally those testing multi-component interventions (e.g. physiotherapy: Bache 2001; Wakefield 2000; Watt 2000) where the interventions were partly or wholly left to the discretion of the therapists. Attempts to standardise were made in some of these more pragmatic trials, which attempt to reflect normal practice (Wakefield 2000a). However, normal practice can vary and there is also the possibility of confounding due to variation in the intervention. The description of the main outcomes was judged insufficient in two trials (Bighea 2013; Christensen 2001) and inadequate in one trial (Rozencwaig 1996). The clinical relevance of reported outcomes, which was mainly based on the reporting of patient-reported outcome measures, was considered only partially relevant in 12 trials and very inadequate in one trial (Rozencwaig 1996). Length of follow-up was considered inadequate in 19 trials. This points to a general failing of the included trials as the short follow-up means that the time was insufficient to ascertain functional recovery fully. In particular, the follow-up of patients to when they are discharged rather than at set times can 
be administratively convenient but could be a source of serious bias. Another very inadequate approach, taken by Cheing 2005, is the timing of final assessment before the time of last treatment.

\section{Further comments on applicability of trial populations}

The types of participants eligible for the individual comparisons are summarised in Table 5, Table 6, Table 7, Table 8 and Table 9. When judging applicability, the definitive treatment (plaster cast immobilisation, internal fixation) and timing in the care pathway (during definitive treatment, post-immobilisation) are key characteristics. It is notable that this update now includes two trials where the definitive treatment was internal fixation (Brehmer 2014; Souer 2011); this reflects the growing, although not strongly evidenced, use of this intervention (Koval 2008). For some trials, however, the study population was further restricted as the trial participants had been referred to physiotherapy (e.g. Jongs 2012; Svensson 1993; Toomey 1986). While trials aimed at the treatment of complications such as CRPS-1 were not included in this review (see Types of participants), participants in Jongs 2012 had wrist contracture and those in Knygsand-Roenhoej 2011 had subacute oedema. The actual trial populations were generally representative in terms of key patient characteristics. Thus most participants were female and middle aged or older (see Table 2). The description of fracture characteristics was usually limited but this aspect is far less critical than in trials of definitive treatment. Notably, half of the trials explicitly excluded patients with serious, usually confirmed, complications (seven explicitly excluded CRPS-1) or comorbidities, or both.

\section{Further comments on applicability of trial interventions}

Evaluation of rehabilitation interventions is difficult to do well. These are generally complex interventions with considerable variation in practice including the often adaptive nature of rehabilitation, where treatment is varied according to the perceived needs and progress of individual patients. These problems are addressed to some extent by pragmatic trials, which aim to evaluate the effects of interventions in real clinical situations, but as explained above, additional problems may result.

An important confounder reflects the personal aspect of these studies; for example, the inter-personal skills of the care provider(s) and motivation of the trial participants could influence the results considerably. There is also the reactive nature of many of these interventions where the basis for progression of, or modification to, the intervention, as well as the timing of completion, is discretionary. The criteria for progression and discharge in most of the included trials were discretionary and though some prior consensus was evident in some of these trials, the criteria were not very specific and could be a major source of variation. For example, participants in Christensen 2001 attended a minimum of 22 therapy sessions, whereas this was the maximum number in Wakefield
2000; yet in both trials the criteria for discharge was basically when the therapist considered no further progress could be made. This highlights that both the immediate and ultimate clinical relevance of the criteria used need to be examined and resolved. Related to this is the reminder in Taylor 1994 that an improvement over the treatment session, as assessed by a therapist, is "of lesser clinical consequence if this improvement is not transferred to a greater rate of improvement over the rehabilitation programme", or indeed in ultimate outcome.

There are also limitations of interpreting comparisons of multicomponent interventions: it is impossible to derive the optimal format of the intervention or the relative effectiveness of its individual components. Changes in definitive treatment may also affect the applicability of the trial results: for instance, in the duration and form of immobilisation, or selection of patients for surgery. The identity of the care provider can also impinge on outcomes as the roles of separate professions, such as occupational therapists and physiotherapists, vary in time and place, but can overlap to a great extent (Smith 2000).

\section{Applicability of the evidence relating to individual comparisons}

As above, the evidence is presented split by rehabilitation started during the definitive treatment period and rehabilitation started post-immobilisation.

\section{Rehabilitation during the definitive treatment period}

The evidence available for rehabilitation starting during the definitive treatment period is summarised in two tables (Table 5; Table 8); there being no trials comparing different rehabilitation interventions.

A key observation relating to the two comparisons testing the provision of specialist therapy during cast immobilisation is that the control group (instructions for self care and exercises during cast immobilisation) of both comparisons points to a minimum of care that is and should be provided. Other observations are the differences in actual interventions constituting formal therapy and the patient populations. One of the two trials finding in favour of the additional formal therapy tested occupational therapy in an older population (Gronlund 1990), whose specific needs may well be greater than the far younger population of Pasila 1974, which did not find additional benefit from supervised exercise.

Very low quality and thus unreliable evidence was available from the four small trials each testing one of four interventions in addition to standard care. These interventions were early digit mobilisation during external fixation, PEMF during cast immobilisation, cyclic pneumatic soft tissue compression using an inflatable cuff during cast immobilisation, and cross-education during cast immobilisation with or without surgical repair. When considering whether further research on these specific interventions is 
warranted, there needs to be assessment of the practical and cost aspects of the intervention and patient acceptability. For example, the intensive nature and potential costs of implementation of the early digit mobilisation intervention (18 sessions of 45 minutes duration over six weeks) tested in Kuo 2013 means that, particularly given the lack of evidence of a clinically important effect of this intervention, further research on this should not be prioritised.

\section{Rehabilitation started post-immobilisation}

The majority of the evidence for rehabilitation applied to the postimmobilisation period. This is summarised in three tables (Table 6; Table 7; Table 9); and include three comparisons of different rehabilitation interventions (see Table 7). As above, the comparisons can be split into those relating to the provision of therapy and those of specific interventions.

Seven comparisons tested the provision or format of therapy after post-immobilisation. The composition and extent of rehabilitation therapy provided by a rehabilitation specialist (usually physiotherapist or occupational therapist) varied considerably. The least of the interventions was a single session of physiotherapy, primarily advice and instructions for a home exercise programme, compared with 'no intervention' after cast removal tested in Kay 2008 (see Table 6). However, the 'control' group of Kay 2008 should not be considered as 'no intervention' as at least some advice would have been provided during cast immobilisation and probably at cast removal. This is still distinct from the systematic advice provided by a rehabilitation specialist who also would have adapted their instructions and exercises where complications were detected. Secondly, the trial involved follow-up assessment at three and six weeks by a rehabilitation specialist that did at minimum allow screening for complications in both groups. As noted above, the intervention group of Kay 2008 is similar to the 'control' group of the four heterogenous trials in the second comparison listed in Table 6. This compared the routine provision of physiotherapy or occupational therapy in addition to instructions for home exercises versus instructions for home exercises from a therapist. Where described the provision of therapy, such as the marked difference in the average attendance between Christensen 2001 and the other three trials (e.g. mean 37 sessions in Christensen 2001 and 4.4 in Maciel 2005), varied considerably in the four trials. The poor description of the interventions hampers the applicability of both Watt 2000, which compared routine physiotherapy (average 5 sessions) versus instructions for home exercises by an orthopaedic surgeon after cast immobilisation, and Bighea 2013, which compared physiotherapy versus a home exercise programme post-cast immobilisation, but as in the previous comparison, a minimum of instructions for home exercises is established. In both trials comparing physiotherapy or occupational therapy versus a progressive home exercise programme after volar plate fixation, there was no mention of home exercises in the supervised therapy group, which is unlikely to reflect usual care. The last comparison of this group evaluated accelerated versus usual rehabilitation after volar plate fixation. There was very low quality evidence of some potentially early benefit in DASH scores that, however, did not persist. In terms of applicability, a particular drawback in the trial evaluating accelerated versus usual rehabilitation after volar plate fixation is the later stoppage time for use of splints and lack of information on advice for home exercises for the control group (Brehmer 2014). The other 11 comparisons were of single interventions in addition to standard care (Table 6; Table 7). The intensity of the interventions ranged from a single five-minute session of ultrasound applied in the clinic (Basso 1998) to a six-hour daily application over eight weeks at home of a dynamic wrist splint (Jongs 2012). Similar observations apply to these interventions as to specific interventions applied during the definitive treatment period. Again, only very low quality and thus unreliable evidence was available from the small trials testing these interventions that, even where suggestive of potential yet limited benefit, should not be used to support their use in practice without further research. Additionally, when considering whether further research on these specific interventions is warranted, there needs to be assessment of the practical and cost aspects of the intervention and patient acceptability. In this case, the inconvenient and demanding nature of the dynamic wrist splint programme, which was poorly adhered to and considered intrusive by some participants, tested in Jongs 2012 means that, particularly given the lack of evidence of a clinically important effect of this intervention, further research on this should not be prioritised.

\section{Quality of the evidence}

The evidence base for this review, formed from 26 small heterogeneous trials, is very limited. As well as in size, the majority of the included trials had methodological shortcomings and were at high risk of bias, usually related to lack of blinding, that could affect the validity of their findings (see Figure 3). There is clearly a need for caution in interpreting the results of small trials which demonstrate 'no evidence of an effect' rather than 'evidence of no effect'. Insufficiencies in quantity and quality of the evidence preclude the drawing of robust conclusions for any of the comparisons evaluated by the included trials.

The assessments based on GRADE of the evidence available for each of the 23 comparisons are presented in the additional tables (Table 5; Table 6; Table 7; Table 8; Table 9). The evidence was typically downgraded one or two levels for study limitations, one or two levels for imprecision and occasionally one level for indirectness. The lack of pooling meant that no downgrading took place for inconsistency or publication bias. The evidence was rated at low quality for two comparisons: physiotherapy or occupational therapy versus control (see Table 6); and physiotherapy or occupational therapy versus progressive home exercise programme after volar plate fixation (see Table 9). The evidence for the other 
comparisons was rated very low quality. Both ratings indicate our uncertainty about the results of these trials.

\section{Potential biases in the review process}

Although our search strategy was comprehensive and without language or publication restrictions, it is very likely that we have missed some trials and findings. In particular, there may be trials that were only reported at conferences, or mixed population trials that included, but did not highlight, patients with wrist fractures. We also point out that pursuing and obtaining unpublished trials and materials is very time-consuming and can be frustrating for both review and trial authors. We are very grateful to all the people who have provided additional information and trial materials. Three aspects of study selection warrant mention. The first applies to the exclusion of three trials that previously we had or would have left awaiting classification. In the unlikely event that further information, such as a full report of the final results (Lohstrater 2006; Woodbridge 2003) or clarification of methods and results (Naik 2007), becomes available we will assess their inclusion in a future update. The second applies to the inclusion of trials whose participants were restricted to those with symptoms, such as of wrist contracture (Jongs 2012) or subacute oedema (Knygsand-Roenhoej 2011). These symptoms are commonplace for patients with these fractures and are likely to have been part of the reason for referral for physiotherapy in other trials. These trials are distinct from excluded trials that either reported only outcomes relating to these symptoms or that focussed on treating complications such as CRPS after wrist fracture. The third relates to the inclusion of Brehmer 2014, which investigated the timing of the start of physiotherapy after volar plate fixation. This acceleration of physiotherapy differs from those trials, which were not eligible, that tested duration of immobilisation after surgery.

\section{Agreements and disagreements with other studies or reviews}

The broad scope of this review with its specific focus on rehabilitation after distal radius fractures continues to be unique. There are systematic reviews that cover specific interventions or comparisons for these fractures or for mixed populations that may or may not include distal radius fractures. Rather than a comprehensive summary of these, a couple of examples are provided here.

Valdes 2014 aimed to compare the effectiveness of a home programme versus a structured therapy programme for patients following distal radius fracture. All seven trials included in Valdes 2014 are included in our review but appear under separate comparisons (Christensen 2001; Kay 2000; Krischak 2009; Maciel 2005; Souer 2011; Wakefield 2000; Watt 2000). Valdes 2014 illustrates our point above that the evidence can be repackaged to address other questions. Nonetheless, they also noted the insufficiency of the evidence from these seven trials, when considered together, to answer their question. A key consideration developed in Valdes 2014 was the representativeness of the study populations, in particular with regard to the inclusion of patients with complications and comorbidities. Although we had already considered this aspect in previous versions of our review, it was in a less formal way and we have now drawn greater attention, as shown in Table 2, to the fact that the majority of the included trials excluded patients with serious complications, in particular CRPS-1, or comorbidities more common in older (> 65 years) patients. However, after definitive treatment, especially cast immobilisation, signs and symptoms such as finger stiffness and swelling are commonplace and it is very likely that patients with these 'complications' were included in most trials investigating interventions at this stage; the exclusions reflecting severe cases with specific, even if still tentative, diagnoses. Related to this are observations in Kay 2008 of an inherent advantage of their study design that facilitated early detection of complications via assessment by a physiotherapist at cast removal and at three and six weeks follow-up. Kay 2008 argued that many of these detected "complications may have been overlooked or have had delayed diagnosis if it were not for the study protocol". Given the high complication rate of these fractures, the finding of a difference between patients and physicians in assessing complications of these fractures by McKay 2001 adds support to the assertion made by Kay 2008. Finally, in terms of the description of interventions, Valdes 2014 noted that only Krischak 2009 referred to a handout that helped guide home exercises.

The second review highlighted here is Katalinic 2010, which investigated the effects of stretch on contractures in people with, or at risk of, contractures. Katalinic 2010 concluded that for all conditions, including people with non-neurological conditions, there is "little or no effect of stretch on pain, spasticity, activity limitation, participation restriction or quality of life". As observed in Table 6, the findings of Jongs 2012 that dynamic splints are unlikely to be therapeutic are consistent with the findings of Katalinic 2010. Several single modality interventions tested for distal radius fractures will have more general applicability and so while this review is purposefully on the rehabilitation of this very common and often problematic fracture, it is very likely that the results from reviews of these modalities on related conditions can help to inform practice should sufficient evidence be available.

\section{A U THORS' CONCLUSIONS}

\section{Implications for practice}

There remains insufficient evidence from RCTs to determine how best to manage the rehabilitation of adults with fractures of the distal radius. Thus the evidence is insufficient to establish exactly what rehabilitation intervention is necessary to optimise functional recovery, or what type of rehabilitation specialists should 
provide this care, or when or for how long this care should be provided, or in what circumstances it should be provided.

The findings of this review should not be construed as a basis for the non-provision of any rehabilitation intervention for people with these injuries. Clearly, general advice and instruction on mobilisation should be given to all patients with these fractures. This is supported also by our assessment of the control groups and standard care provided to all participants of the included trials. There is also a case, albeit based on very low quality evidence, for the specialist assessment and tailored advice and instruction for home exercises soon after cast removal. Although patients with serious complications or comorbidities were excluded in many of the included trials, it is also clear that additional therapy will be necessary for patients with complications or serious functional impairment. Whilst many people with these fractures will make a satisfactory recovery, it should be remembered that the consequences of a bad outcome might include disabling pain (Fisk 1991), loss of independence (Scaf-Klomp 2001) and that, for many patients, these fractures indicate an increased risk of further fracture in the future (Senanayake 2001). Furthermore, Edwards 2010 showed that these fractures contribute to clinically important functional decline in older women (age > 65 years).

\section{Implications for research}

Further research is warranted to identify effective rehabilitation interventions for these common fractures in adults. This is supported too by a research prioritisation exercise, conducted in 2003 and informed by the evidence available in Cochrane reviews at that time, that established three priority research areas to identify what rehabilitation interventions should be given a) during and b) postimmobilisation for conservatively treated fractures and "what are good (practical and effective) ways of (routinely) delivering rehabilitative interventions?" (Handoll 2003b). Research on rehabilitation interventions was also identified in the American Academy of Orthopaedic Surgeons distal radius fracture guidelines (AAOS 2009). This highlighted research on "physical therapy (e.g. early formal physical therapy, self supervised home programs)". However, the identification of priority questions for research requires further refinement and such an exercise should take into account the current coverage of the evidence, including the topics covered in the ongoing trials, current practice and differences in practice, and should involve consultation with patients as to their preferences and values. Achieving professional consensus on treatment uncertainties should facilitate sufficient recruitment into multicentre trials and also implementation of their findings.

As before, we consider that a priority area is an examination of the provision, mode and format of advice and instruction for home exercises, both during the definitive treatment period, and postimmobilisation. Research would also be worthwhile to identify interim and intermediate functional outcomes, which correlate with long-term outcome and which can be used to indicate the need for more extensive rehabilitation, and act as criteria for progressing and discharging people from rehabilitation. These research aims need good quality, generally applicable evidence from methodologically sound and sufficiently powered RCTs, preferably multicentred. These trials require easily applied standardised materials, comprehensive assessment of outcome with the use of validated measures, and long-term follow-up.

Consideration should also be given to the potential differences in impact of rehabilitation in different participant groups and circumstances. This includes older people with comorbidities and who are at greater risk of functional decline.

\section{ACKNOWLEDGEMENTS}

We thank the following for their help and feedback at the editorial and external review of this version of the review: Lindsey Elstub, Nigel Hanchard, Ann Lucado and Janet Wale.

We are very grateful to the following for supplying us with additional information on their trials and other insights: Sarah Bache, Gladys Cheing, Filiz Can, Ole Maagaard Christensen, Susan Fortier, Bo Grunlund, Louise Hiller, Sandra Kay, Li-Chieh Kuo, Richard Rozencwaig, Micheal Schuler, Nick Taylor, Alison Wakefield and Sarah Woodbridge.

This section of the previous versions of the review (Handoll 2006) contains the acknowledgements for the protocol up to the previous version; only our acknowledgements to trial authors are retained here. We are grateful to Rajan Madhok and Tracey Howe for their contributions to the protocol and all versions of the review up to Issue 3, 2006. Furthermore, we are grateful to Tracey Howe for her initial help with screening and risk of bias assessment and data extraction of two newly included trials in 2013.

This review was supported by the National Institute for Health Research (NIHR) via Cochrane Infrastructure funding to the Cochrane Bone, Joint and Muscle Trauma Group. The views and opinions expressed therein are those of the authors and do not necessarily reflect those of the Systematic Reviews Programme, NIHR, National Health Service (NHS) or the Department of Health. 


\section{R E F E R E N C E S}

\section{References to studies included in this review}

Bache 2001 \{published and unpublished data\}

Bache SJ. The effective two different physiotherapy approaches to rehabilitation on outcome of patients with distal radial fractures. In: The National Research Register, Issue 1, 2001. Oxford: Update Software.

* Bache SJ, Ankcorn L, Hiller L, Gaffrey A. Two different approaches to physiotherapeutic management of patients with distal radial fractures [abstract]. Physiotherapy 2000;86 (7):383.

Bache SJ, Hiller L, Bache CE. A pragmatic randomised controlled trial of two different approaches to the physiotherapeutic management of patients with distal radial fractures. [Paper in preparation for journal submission].

Basso 1998 \{published data only\}

Basso O, Pike JM. The effect of low frequency, long-wave ultrasound therapy on joint mobility and rehabilitation after wrist fracture. Journal of Hand Surgery - British Volume 1998;23(1):136-9.

Bighea 2013 \{published data only\}

Bighea AC, Patru S, Marcu IR, Popescu R. Supervised and unsupervised rehabilitation after osteoporotic wrist fracture (abstract). Osteoporosis International 2013;24(Suppl 1): S152.

Brehmer 2014 \{published data only\} Brehmer JL, Husband JB. Accelerated rehabilitation compared with a standard protocol after distal radial fractures treated with volar open reduction and internal fixation: A prospective, randomized, controlled study. Journal of Bone and Joint Surgery. American Volume 2014;96 (19):1621-30.

\section{Challis 2007 \{published data only\}}

Challis MJ, Jull GJ, Stanton WR, Welsh MK. Cyclic pneumatic soft-tissue compression enhances recovery following fracture of the distal radius: a randomised controlled trial. Australian Journal of Physiotherapy 2007;53 (4):247-52.

\section{Cheing 2005 \{published data only\}}

Cheing GL. [personal communication] 9 December 2005.

* Cheing GL, Wan JW, Kai Lo S. Ice and pulsed electromagnetic field to reduce pain and swelling after distal radius fractures. Journal of Rehabilitation Medicine 2005;37 (6):372-7.

Christensen 2001 \{published and unpublished data\} Christensen OM, Kunov A, Hansen FF, Christiansen TC, Krasheninnikoff M. Occupational therapy and Colles' fractures. International Orthopaedics 2001;25(1):43-5.

Cooper 2001 \{published and unpublished data\} Cooper A. [personal communication] 7 November 2005. Cooper A. Early therapeutic intervention following fractured distal radius [abstract]. Proceedings of The
Chartered Society of Physiotherapy Annual Congress and Exhibition; 2004 Oct 8-10; Birmingham (UK). 2004. * Cooper AJ. The Effects of Early Intervention for Patients Following Fractured Distal Radius [MSc thesis]. Derby: University of Derby, 2001.

Gronlund 1990 \{published and unpublished data\} Gronlund B, Harreby MS, Kofoed R, Rasmussen L. The importance of early exercise therapy in the treatment of Colles' fracture. A clinically controlled study [Betydningen af tidlig ergoterapi ved behandling af Colles-fraktur. En klinisk kontrolleret undersogelse]. Ugeskrift for Laeger 1990; 152(35):2491-3.

\section{Jongs 2012 \{published data only\}}

Jongs R, Harvey L. Do dynamic splints reduce wrist stiffness following fracture?. www.anzctr.org.au/Trial/Registration/ TrialReview.aspx?id=82911 (accessed 5 April 2013 and 12 February 15)

* Jongs RA, Harvey LA, Lucas BR. Dynamic splints do not reduce contracture following distal radial fracture: a randomised controlled trial. Journal of Physiotherapy 2012; 58(3):173-80.

Kay 2000 \{published data only\}

Kay S, Haensel N, Stiller K. An evaluation of the effect of passive mobilisation techniques on wrist range, pain and function following fracture of the distal radius [abstract]. Australian Hand Therapy Association Conference; 1998 Oct 15-18; Perth (Western Australia). 1998.

Kay S, Haensel N, Stiller K. Passive mobilisation: Its effect following distal radius fracture [abstract]. South Australian Hand Surgery Society Meeting; 1998 Nov 20; Adelaide (South Australia). 1998.

* Kay S, Haensel N, Stiller K. The effect of passive mobilisation following fractures involving the distal radius: a randomised study. Australian Journal of Physiotherapy 2000;46(2):93-101.

Kay 2008 \{published data only\}

Kay S. [personal communication] 17 January 2003.

Kay S. [personal communication] 23 February 2004. Kay S. [personal communication] 27 May 2015. Kay S. [personal communication] 27 October 2005. Kay $S$. The effect of an advice and exercise program on patients with distal radius fractures. http:// www.anzctr.org.au/ACTRN12607000204448.aspx (accessed 22 February 2015).

* Kay S, McMahon M, Stiller K. An advice and exercise program has some benefits over natural recovery after distal radius fracture: a randomised trial. Australian Journal of Physiotherapy 2008;54(4):253-9.

\section{Knygsand-Roenhoej 2011 \{published data only\}} Knygsand-Roenhoej K, Maribo T. A randomized clinical controlled study comparing the effect of modified manual edema mobilization treatment with traditional edema technique in patients with a fracture of the distal radius. Journal of Hand Therapy 2011;24(3):184-93. 
Krischak 2009 \{published data only\}

Krischak GD, Krasteva A, Schneider F, Gulkin D, Gebhard F, Kramer M. Physiotherapy after volar plating of wrist fractures is effective using a home exercise program. Archives of Physical Medicine and Rehabilitation 2009;90(4):537-44.

Kuo 2013 \{published data only\}

Kuo LC. [personal communication] 1 April 2015.

* Kuo LC, Yang TH, Hsu YY, Wu PT, Lin CL, Hsu HY, et al. Is progressive early digit mobilization intervention beneficial for patients with external fixation of distal radius fracture? A pilot randomized controlled trial. Clinical Rehabilitation 2013;27(11):983-93.

Lazovic 2012 \{published data only\}

Lazovic M, Kocic M, Dimitrijevic L, Stankovic I, Spalevic M, Ciric T. Pulsed electromagnetic field during cast immobilization in postmenopausal women with Colles' fracture. Srpski Arhiv Za Celokupno Lekarstvo 2012;140(910):619-24.

Maciel 2005 \{published and unpublished data\} * Maciel JS, Taylor NF, McIlveen C. A randomised clinical trial of activity-focussed physiotherapy on patients with distal radius fractures. Archives of Orthopaedic and Trauma Surgery 2005;125(8):515-20.

Taylor N. [personal communication] 15 November 2002. Taylor N. [personal communication] 9 February 2004.

Magnus 2013 \{published data only\}

Magnus C, Johnston G, Arnold C, Dal Bello-Haas V, Basran J, Krentz J, et al. Fractures of the distal radius In women in middle and older adulthood: The CAST Trial - preliminary results of a randomised pilot study. Canadian Orthopaedic Association Annual Meeting 2012, Ottawa, Canada http:/ /www.coa-aco.org/images/stories/meetings/ottawa'2012/ COA' $2012{ }^{\circ}$ Ottawa ${ }^{\circ}$ ON ${ }^{*}$ Abstract' Supplement.pdf (accessed 19/01/2015).

* Magnus CR, Arnold CM, Johnston G, Dal-Bello Haas V, Basran J, Krentz JR, et al. Cross-education for improving strength and mobility after distal radius fractures: a randomized controlled trial. Archives of Physical Medicine and Rehabilitation 2013;94(7):1247-55.

Pasila 1974 \{published data only\} Pasila M, Karahapju EO, Lepisto PV. Role of physical therapy in the recovery of function after Colles' fracture. Archives of Physical Medicine and Rehabilitation 1974;55(3): $130-4$.

Rozencwaig 1996 \{published and unpublished data\} Rozencwaig R, Kaye JJ, Gravois D, Fortier S. Efficacy of continuous passive motion after external fixation of unstable distal radius fractures in reducing amount of therapeutic intervention required to return to daily activities and employment [abstract]. Orthopaedic Transactions 1996;20 (4):879.

Souer 2011 \{published data only\}

Ring D. Comparison of occupational therapy and home exercises for adults with operatively treated distal radius fractures. http://clinicaltrials.gov/show/NCT00438750

(accessed 8 March 2013 and 15 February 2015).

* Souer JS, Buijze G, Ring D. A prospective randomized controlled trial comparing occupational therapy with independent exercises after volar plate fixation of a fracture of the distal part of the radius. Journal of Bone and Joint Surgery - American Volume 2011;93(19):1761-6.

Souer S, Ring D, Buijze GA. A prospective randomized controlled trial comparing occupational therapy with independent exercises after volar plate fixation of a fracture of the distal radius (abstract). Journal of Hand Surgery 2011; 38(8 Suppl): 13 .

\section{Svensson 1993 \{published data only\}}

Svensson BH, Frellsen MB, Basse PN, Bliddal H, Caspers J, Parby K. Effect of pneumatic compression in connection with ergotherapeutic treatment of Colles' fracture. A clinical controlled trial [Effekten af trykbandagebehandling i forbindelse med ergoterapeutisk behandling af Colles-fraktur. En klinisk kontrolleret undersogelse]. Ugeskrift for Laeger 1993;155(7):463-6.

Taylor 1994 \{published and unpublished data\} Taylor NF, Bennell KL. The effectiveness of passive joint mobilisation on the return of active wrist extension following Colles' fracture: a clinical trial. New Zealand Journal of Physiotherapy 1994;22(1):24-8.

Toomey 1986 \{published data only\}

Toomey R, Grief Schwartz R, Piper MC. Clinical evaluation of the effects of whirlpool on patients with Colles' fractures. Physiotherapy Canada 1986;38(5):280-4.

Wakefield 2000 \{published and unpublished data\} Wakefield AE, McQueen MM. The role of physiotherapy and clinical predictors of outcome after fracture of the distal radius. Journal of Bone and Joint Surgery - British Volume 2000;82(7):972-6.

Watt 2000 \{published and unpublished data\} Watt CF, Taylor NF, Baskus K. Do Colles' fracture patients benefit from routine referral to physiotherapy following cast removal?. Archives of Orthopaedic and Traumatic Surgery 2000;120(7-8):413-5.

\section{References to studies excluded from this review}

\section{ACTRN12606000160538 \{unpublished data only\}}

Goldbloom D, Blackmore S. Aggressive mobilisation following fragment specific fixation of distal radius fractures. www.anzctr.org.au/ACTRN12606000160538.aspx (accessed 12 February 2015).

Ayhan 2014 \{published data only\} Ayhan C, Unal E, Yahut Y. Core stabilisation reduces compensatory movement patterns in patients with injury to the arm: a randomized controlled trial. Clinical Rehabilitation 2014;28(1):36-47.

\section{Bünger 2011 \{published data only\}}

Bünger A, Krägenow $\mathrm{H}$. Concept of a randomized controlled pilot study on the "effectiveness of mirror therapy in distal radius fracture" [Erfahrungsbericht über die Planung und 
Durchführung einer randomisiert-kontrollierten Pilotstudie zur Thematik "Wirksamkeit der Spiegeltherapie bei distaler Radiusfraktur"]. Ergoscience 2011;4:194-6.

\section{Can 2001 \{published data only\}}

Can F. [personal communication] 17 January 2003.

* Can F, Erden Z, Yuceturk A. The effect of manual therapy in the rehabilitation of distal radius fractures [Turkish]. Fizyoterapi Rehabilitasyon 2001;12(3):99-104.

Coyle 1998 \{published data only\}

Coyle JA, Robertson VJ. Comparison of two passive mobilizing techniques following Colles' fracture: a multielement design. Manual Therapy 1998;3(1):34-41.

Haren 2000 \{published data only\}

Haren K, Backman C, Wiberg M. Effect of manual lymph drainage as described by Vodder on oedema of the hand after fracture of the distal radius: A prospective clinical study. Scandinavian Journal of Plastic and Reconstructive Surgery and Hand Surgery 2000;34(4):367-72.

Haren 2004 \{published data only\}

${ }^{*}$ Haren K, Wiberg M. A prospective randomized controlled trial of manual lymph drainage (MLD) for the reduction of hand oedema distal radius fracture. British Journal of Hand Therapy 2006;11(2):41-7.

Haren K, Wiberg M. The effect of manual lymph drainage on oedema of the hand after fracture of the distal radius [abstract]. British Journal of Hand Therapy 2004;9(2):55-6.

Hunt 2001 \{published and unpublished data\}

Hunt J. Evaluation of a functional treatment programme for patients following a fractured distal radius: a pilot study. In: The National Research Register, Issue 1, 2001. Oxford: Update Software.

Jarvis 2001 \{published and unpublished data\}

Jarvis RE. Comparison of treatments for Colles fracture. In: The National Research Register, Issue 1, 2001. Oxford: Update Software.

Kingston 2014 \{published and unpublished data\}

Kingston G, Gray M. A clinical trial to investigate whether providing a home exercise program on DVD improves compliance with patients who have hand injuries. www.anzctr.org.au/ACTRN12608000530325.aspx (accessed 15 February 2015).

* Kingston GA, Williams G, Gray MA, Judd J. Does a DVD improve compliance with home exercise programs for people who have sustained a traumatic hand injury? Results of a feasibility study. Disability and Rehabilitation: Assistive Technology 2014;9(3):188-94.

Lohstrater 2006 \{published data only\}

Lohstrater A, Bak P. Efficiency and cost-effectiveness of the rehabilitation management of the VBG in patients after distal radius fracture [Medizinische und "konomische effizienz des reha-managements der VBG bei patienten nach distaler radiusfraktur]. Physikalische Medizin Rehabilitationsmedizin Kurortmedizin 2006;16(3):155-9.
Naik 2007 \{published data only\}

Chitra J. [personal correspondence] 3 March 2013.

* Naik VC, Chitra J, Khatri S. Effectiveness of Maitland versus Mulligan mobilization technique following post surgical management of colles' - fracture - RCT. Indian Journal of Physiotherapy and Occupational Therapy 2007;1 (4):14-8.

Neeman 1988 \{published data only\}

Neeman RL. Orthokinetic orthoses application in treatment of patients with Colles' fracture post-immobilization hypokinesia: A single-subject design efficacy study. Physiotherapy Canada 1988;40(5):286-95.

\section{Nikolova 1969 \{published data only\}}

Nikolova L. Physical therapy in the rehabilitation of bone fracture complications [Physiotherapeutische rehabilitation bei knochenbruchkomplikationen]. Munchener Medizinische Wochenschrift 1969;111(11): 592-9.

Oskarsson 1997 \{published data only\} Oskarsson GV, Hjall A, Aaser P. Physiotherapy: an overestimated factor in after-treatment of fractures in the distal radius?. Archives of Orthopaedic and Trauma Surgery 1997;116(6-7):373-5.

\section{Pasila 1980 \{published data only\}}

Pasila M, Sundholm A. Mobilization of stiff hands after 104 Colles' fractures: a comparison between the value of the Movelat and its base cream. Rheumatology and Rehabilitation 1980;19(3):170-2.

Ramesh 1998 \{published data only\}

* Ramesh M, Morrissey B, Healy JB, Roy-Choudhury S, Macey AC. Effectiveness of the A-V impulse hand pump. Journal of Bone and Joint Surgery - British Volume 1999;81 (2):229-33.

Ramesh M, Morrissey B, Roy-Choudhury S, Ajaml M, Healy JB, Macey AC. Effectiveness of the A.V. impulse hand pump [abstract]. Journal of Bone and Joint Surgery British Volume 1998;80 Suppl 1:69.

\section{Rodrick 2004 \{published data only\}}

Rodrick JR, Howard S. Sub-acute edema reduction in patients with wrist disorders: a pilot study comparing the effectiveness of traditional retrograde massage versus manual edema mobilization [abstract]. British Journal of Hand Therapy 2004;9(2):54-5.

Schwartz-Jensen 2002 \{published data only\} Schwartz Jensen K. Pilot study of the effect of individual occupational therapy given to patients with a distal radial fracture during the immobilization period [abstract]. Journal of Hand Surgery - British Volume 2002;27 Suppl 1: 70.

Wang 2012 \{published data only\}

Wang GJ, Liu J. Clinical randomized controlled trial on ultrashort wave and magnetic therapy for the treatment of early stage distal radius fractures [Chinese]. Zhongguo Gushang 2012;25(7):572-5. 
Woodbridge 2003 \{unpublished data only\}

Woodbridge S. [personal communication] 23 February 2004.

Woodbridge S. [personal communication] 23 January 2003. Woodbridge S. [personal communication] 9 November 2005.

Zhang 2005 \{published data only\}

Zhang H-F, Wang J-B, Zhao Q-L, Yang S-P, Zhao M$\mathrm{J}$. Effect of gripping exercise on the radius bone mass of patients with Colles fracture. Zhongguo Linchuang Kangfu [Chinese Journal of Clinical Rehabilitation] 2005;9(8): 136-7.

\section{References to studies awaiting assessment}

Duvoric 2005 \{published data only\}

Durovic A, Zivotic-Vanovic M, Railic Z. Effects of circumferential rigid wrist orthoses in rehabilitation of patients with radius fracture at typical site [Serbian]. Vojnosanitetski Pregled: Military Medical and Pharmaceutical Review 2005;62(4):257-64.

McPhate 1998 \{published data only\}

Brock KA. [personal communication] 5 December 2005. Kay S. [personal communication] 27 October 2005. McPhate M, Robertson VJ. Passive mobilisation in the physiotherapy treatment of Colles fracture [abstract]. Proceedings of the Tenth Biennial Conference of the Manipulative Physiotherapists Association of Australia; 1987 Nov 26-29; Melbourne (Australia). St Kilda, Victoria, Australia: Manipulative Physiotherapists Association of Australia, 1997:121-2.

* McPhate M, Robertson VJ. Physiotherapy treatment of Colles fractures: hands off or hands on [abstract]. Proceedings of the Fifth International Australian Physiotherapy Association Congress; 1998 May 11; Hobart (Australia). 1998:235.

NCT00816998 \{unpublished data only\} Shin AY. Range of motion and patients with distal radius fractures. https://clinicaltrials.gov/show/NCT00816998 (accessed 15 February 2015).

NCT01262807 \{unpublished data only\} Dubberley J, Leiter J, Wittmeier K. Reducing range of motion deficits post radial fracture. https://clinicaltrials.gov/ ct2/show/NCT01262807 (accessed 2 March 2015).

NCT01589627 \{unpublished data only\}

Berner S. Wrist Extension Dynasplint (WED) distal radius fracture. https://clinicaltrials.gov/show/NCT01589627 (accessed 12 February 12)

Oken 2011 \{published data only\}

Oken O, Cecel E, Oken FO, Yorgancioglu RZ. Hospitalbased versus home-based program in rehabilitation of distal radius fractures. Turkish Journal of Physical Medicine \& Rehabilitation/Turkiye Fiziksel Tip ve Rehabilitasyon Dergisi 2011;57(3):139-42.

Schmidt 2013 \{published data only\}

Schmidt J, Tessmann UJ, Schmidt I. Compression glove has advantages in the functional aftercare of distal radius fractures [Der Kompressionshandschuh hat Vorteile in der funktionellen Nachbehandlung der distalen Radiusfraktur]. Zeitschrift fur Orthopadie und Unfallchirurgie 2013;151(1): 80-4.

\section{References to ongoing studies}

\section{ACTRN12612000118808 \{unpublished data only\}}

Bruder A. Does exercise following distal radius fracture improve activity? A multi-centre randomised controlled trial. http://www.anzctr.org.au/ACTRN12612000118808.aspx (accessed 12 February 2015).

JPRN-UMIN000015003 \{unpublished data only\} Gamo K. Effectiveness of occupational therapy after volar locking plate fixation of the distal radius fracture. A prospective randomized controlled trial. http:// apps.who.int/trialsearch/Trial2.aspx?TrialID=JPRNUMIN000015003 (accessed 15 February 2015).

NCT01118715 \{unpublished data only\}

* Harris L, Cole A, Monroe P, Chancey J, Shuler M. Compression glove may reduce complications secondary to distal radius fracture (abstract). Journal of Hand Therapy 2011;24(4):383-4.

Schuler M. [personal communication] 1 June 2015. Schuler M. Use of compression glove to prevent complications after distal radius fractures: a randomized controlled trial. https://clinicaltrials.gov/show/ NCT01118715 (accessed February 12 2015).

\section{NCT01394809 \{unpublished data only\}}

Korbus $\mathrm{H}$, Schott $\mathrm{N}$. Effects of motor cognitive training on functional loss after osteoporotic wrist fractures (PROFinDTP4). http://clinicaltrials.gov/show/NCT01394809 (accessed 3 August 2013).

* Schott N, Korbus H. Preventing functional loss during immobilization after osteoporotic wrist fractures in elderly patients: a randomized clinical trial. BMC Musculoskeletal Disorders 2014;15:287.

\section{NCT01518179 \{unpublished data only\}}

Bernfeld B, Milman U, Schreuer N, Miler I. Compression gloves for distal radius fracture. https:/clinicaltrials.gov/ show/NCT01518179 (accessed 12 February 2015).

\section{NCT01693094 \{unpublished data only\}}

Ring D. A randomized trial measuring the effect of decision aids on patients' satisfaction, conflict of decision-making and clinical outcome. https://clinicaltrials.gov/show/ NCT01693094 (accessed 12 February 2015).

\section{NCT01921062 \{unpublished data only\}}

Broekstra DC, Stenekes MW. Motor imagery in rehabilitation after a distal radius fracture (MIDRF). https: //clinicaltrials.gov/show/NCT01921062 (accessed 12 February 2015)

\section{NCT02015468 \{unpublished data only\}}

Hammer O-L, Madsen JE. The value of early mobilization and physiotherapy following wrist fractures treated by volar plating. https://clinicaltrials.gov/show/NCT02015468 (accessed 12 February 2015). 


\section{Additional references}

\section{Atkins 1996}

Atkins RM. Post-traumatic reflex sympathetic dystrophy. In: Ficat CCJ editor(s). Balliere's Clinical Orthopaedics Reflex Sympathetic Dystrophy. Vol. 1, London: Bailliere Tindall, 1996:223-40.

Chen 2010

Chen C, Bode R. Psychometric validation of the Manual Ability Measure-36 (MAM-36) in patients with neurologic and musculoskeletal disorders. Archives of Physical Medicine and Rehabilitation 2010;91(3):414-20.

\section{Chitnavis 1999}

Chitnavis J. The wrist. In: Pynsent PB, Fairbank JCT, Carr AJ editor(s). Classification of musculoskeletal trauma. Oxford: Butterworth Heinemann, 1999:146-70.

\section{Collins 1993}

Collins DC. Management and rehabilitation of distal radius fractures. Orthopedic Clinics of North America 1993;24(2): $365-78$.

\section{Cooney 1980}

Cooney WP 3rd, Dobyns JH, Linscheid RL. Complications of Colles' fractures. Journal of Bone and Joint Surgery American Volume 1980;62(4):613-9.

\section{Costa 2015}

Costa ML, Achten J, Plant C, Parsons NR, Rangan A, Tubeuf $S$, et al. UK DRAFFT: a randomised controlled trial of percutaneous fixation with Kirschner wires versus volar locking-plate fixation in the treatment of adult patients with a dorsally displaced fracture of the distal radius. Health Technology Assessment 2015;19:17. [DOI: 10.3310/ hta19170]

\section{Court-Brown 2006}

Court-Brown CM, Caesar B. Epidemiology of adult fractures: A review. Injury 2006;37(8):691-7.

\section{Cummings 1985}

Cummings SR, Kelsey JL, Nevitt MC, O’Dowd KJ. Epidemiology of osteoporosis and osteoporotic fractures. Epidemiologic Reviews 1985;7:178-208.

\section{Deeks 2011}

Deeks JJ, Higgins JPT, Altman DG (editors). Chapter 9: Analysing data and undertaking meta-analyses. In: Higgins JPT, Green S (editors). Cochrane Handbook for Systematic Reviews of Interventions Version 5.1.0 (updated March 2011). The Cochrane Collaboration, 2011. Available from www.cochrane-handbook.org.

\section{Edwards 2010}

Edwards BJ, Song J, Dunlop DD, Fink HA, Cauley JA. Functional decline after incident wrist fractures - Study of Osteoporotic Fractures: prospective cohort study. BMJ 2010;341:c3324.

Fisk 1991

Fisk GR. The painful wrist: problems and solutions. In: Wynn Parry CB editor(s). Management of Pain in the Hand and Wrist. Edinburgh: Churchill Livingstone, 1991:48-68.

\section{Gartland 1951}

Gartland JJ, Werley CW. Evaluation of healed Colles' fractures. Journal of Bone and Joint Surgery - American Volume 1951;33(4):895-910.

Gronlund 2001

Gronlund B. [personal communication] 8 August 2001.

Handoll 2003a

Handoll HHG, Madhok R. Surgical interventions for treating distal radial fractures in adults. Cochrane Database of Systematic Reviews 2003, Issue 3.

\section{Handoll 2003b}

Handoll HH, Madhok R. From evidence to best practice in the management of fractures of the distal radius in adults: working towards a research agenda. BMC Musculoskeletal Disorders 2003;4:27.

\section{Handoll 2003c}

Handoll HHG, Madhok R. Conservative interventions for treating distal radial fractures in adults. Cochrane Database of Systematic Reviews 2003, Issue 2. [DOI: 10.1002/ 14651858.CD000314]

\section{Handoll 2007}

Percutaneous pinning for treating distal radial fractures in adults. Handoll HHG, Vaghela MV, Madhok R. Cochrane Database of Systematic Reviews 2007, Issue 3. [DOI: 10.1002/14651858.CD006080.pub2]

\section{Harris 2011}

Harris L, Cole A, Monroe P, Chancey J, Shuler M. Compression glove may reduce complications secondary to distal radius fracture (abstract). Journal of Hand Therapy 2011;24(4):383-4.

\section{Higgins 2003}

Higgins JPT, Thompson SG, Deeks JJ, Altman DG. Measuring inconsistency in meta-analyses. BMJ 2003;327: 557-60.

\section{Higgins 2011a}

Higgins JPT, Green S (editors). Cochrane Handbook for Systematic Reviews of Interventions Version 5.1.0 [updated March 2011]. The Cochrane Collaboration, 2011. Available from www.cochrane-handbook.org.

\section{Higgins 2011b}

Higgins JPT, Altman DG, Sterne JAC (editors). Chapter 8: Assessing risk of bias in included studies. In: Higgins JPT, Green S (editors). Cochrane Handbook for Systematic Reviews of Interventions Version 5.1.0 [updated March 2011]. The Cochrane Collaboration, 2011. Available from www.cochrane-handbook.org.

\section{Hoare 2014}

Hoare CP, Dickson DR, Armstrong DJ, Nuttall D, Watts AC. Internal fixation for treating distal radius fractures in adults. Cochrane Database of Systematic Reviews 2014, Issue 7. [DOI: $10.1002 / 14651858 . C D 011213]$

\section{HSCIC 2015}

Health and Social Care Information Centre. NHS Business Definitions. http://www.datadictionary.nhs.uk/ data 'dictionary/nhs' business' definitions/f/ 
first 'definitive treatment 'de.asp?shownav=1 (accessed 2 September 2015).

\section{Katalinic 2010}

Katalinic OM, Harvey LA, Herbert RD, Moseley AM, Lannin NA, Schurr K. Stretch for the treatment and prevention of contractures. Cochrane Database of Systematic Reviews 2010, Issue 9. [DOI: 10.1002/ 14651858.CD007455.pub2]

\section{Koval 2008}

Koval KJ, Harrast JJ, Anglen JO, Weinstein JN. Fractures of the distal part of the radius: The evolution of practice over time. Where's the evidence?. Journal of Bone and Joint Surgery - Series A 2008;90(9):1855-61.

\section{Lefebvre 2011}

Lefebvre C, Manheimer E, Glanville J. Chapter 6: Searching for studies. In: Higgins JPT, Green S (editors). Cochrane Handbook for Systematic Reviews of Interventions Version 5.1.0 [updated March 2011]. The Cochrane Collaboration, 2011. Available from www.cochrane-handbook.org.

\section{Levine 1993}

Levine DW, Simmons BP, Koris MJ, Daltroy LH, Hohl GG, Fossel AH, et al. A self-administered questionnaire for the assessment of severity of symptoms and functional status in carpal tunnel syndrome. Journal of Bone and Joint Surgery - American Volume 1993;75(11):1585-92.

\section{MacDermid 2000}

MacDermid JC, Richards RS, Donner A, Bellamy N, Roth $\mathrm{JH}$. Responsiveness of the short form-36, disability of the arm, shoulder, and hand questionnaire, patient-rated wrist evaluation, and physical impairment measurements in evaluating recovery after a distal radius fracture. Journal of Hand Surgery - American Volume 2000;25(2):330-40.

\section{Mattila 2011}

Mattila VM, Huttunen TT, Sillanpää P, Niemi S, Pihlajamäki H, Kannus P. Significant change in the surgical treatment of distal radius fractures: a nationwide study between 1998 and 2008 in Finland. Journal of Trauma 2011;71(4):939-42; discussion 942-3.

\section{McKay 2001}

Mckay SD, MacDermid JC, Roth JH, Richards RS. Assessment of complications of distal radius fractures and development of a complication checklist. Journal of Hand Surgery - American Volume 2001;26(5):916-22.

\section{Moher 2009}

Moher D, Liberati A, Tetzlaff J, Altman DG, The PRISMA Group. Preferred reporting items for systematic reviews and meta-analyses: The PRISMA Statement. BMJ 2009;339: 2535.

Nellans 2012

Nellans KW, Kowalski E, Chung KC. The epidemiology of distal radius fractures. Hand Clinics 2012;28(2):113-25.

\section{Nguyen 2001}

Nguyen TV, Center JR, Sambrook PN, Eisman JA. Risk factors for proximal humerus, forearm, and wrist fractures in elderly men and women. The Dubbo Osteoporosis
Epidemiology Study. American Journal of Epidemiology

2001;153(6):587-95.

\section{O'Neill 2001}

O'Neill TW, Cooper C, Finn JD, Lunt M, Purdie D, Reid $\mathrm{DM}$, et al. Incidence of distal forearm fracture in British men and women. Osteoporosis International 2001;12(7): 555-8.

RevMan 2014

The Nordic Cochrane Centre, The Cochrane Collaboration. Review Manager (RevMan). 5.3. Copenhagen: The Nordic Cochrane Centre, The Cochrane Collaboration, 2014.

\section{Sahlin 1990}

Sahlin Y. Occurrence of fractures in a defined population: a 1-year study. Injury 1990;21(3):158-60.

\section{Scaf-Klomp 2001}

Scaf-Klomp W, van Sonderen E, Danderman R, Ormel J, Kempen GIJM. Recovery of physical function after limb injuries in independent older people living at home. Age and Ageing 2001;30(3):213-9.

\section{Schmitt 2004}

Schmitt JS, Di Fabio RP. Reliable change and minimum important difference (MID) proportions facilitated group responsiveness comparisons using individual threshold criteria. Journal of Clinical Epidemiology 2004;57(10): 1008-18.

\section{Schunemann 2011}

Schunemann HJ, Oxman AD, Vist GE, Higgins JPT, Deeks JJ, Glaziou P, et al. Chapter 12: Interpreting results and drawing conclusions. In: Higgins JPT, Green S (editors). Cochrane Handbook for Systematic Reviews of Interventions Version 5.1.0 [updated March 2011]. The Cochrane Collaboration, 2011. Available from www.cochrane-handbook.org.

Senanayake 2001

Senanayake $S$, Francis RM. Distal forearm fracture - time for action? [Editorial]. Age and Ageing 2001;30:187-8.

\section{Sheehan 1983}

Sheehan NJ, Sheldon F, Marks D. Grip strength and torquometry in the assessment of hand function in patients with rheumatoid arthritis. British Journal of Rheumatology 1983;22(3):158-64.

\section{Singer 1998}

Singer BR, McLauchlan GJ, Robinson CM, Christie J. Epidemiology of fractures in 15,000 adults: the influence of age and gender. Journal of Bone and Joint Surgery - British Volume 1998;80(2):243-8.

\section{Smith 2000}

Smith S, Roberts P, Balmer S. Role overlap and professional boundaries: Future implications for physiotherapy and occupational therapy in the NHS. Physiotherapy 2000;86 (8):397-400.

\section{Solgaard 1988}

Solgaard S. Function after distal radius fracture. Acta Orthopaedica Scandinavica 1988;59(1):39-42. 


\section{Sorensen 2013}

Sorensen AA, Howard D, Tan WH, Ketchersid J, Calfee RP. Minimal clinically important differences of three patientrated outcomes instruments. Journal of Hand Surgery 2013; 38(4):641-9.

\section{Stewart 1984}

Stewart HD, Innes AR, Burke FD. Functional cast-bracing for Colles' fractures. A comparison between cast-bracing and conventional plaster casts. Journal of Bone and Joint Surgery - British Volume 1984;66(5):749-53.

\section{Valdes 2014}

Valdes K, Naughton N, Michlovitz S. Therapist supervised clinic-based therapy versus instruction in a home program following distal radius fracture: a systematic review. Journal of Hand Therapy 2014;27(3):165-73.

\section{Wakefield 2000a}

Wakefield AE. Evidence-based physiotherapy: The case for pragmatic randomised controlled trials. Physiotherapy 2000; 86(8):394-6.

Walenkamp 2015

Walenkamp MM, de Muinck Keizer RJ, Goslings JC, Vos LM, Rosenwasser MP, Schep NW. The Minimum Clinically Important Difference of the Patient-rated Wrist Evaluation Score for patients with distal radius fractures. Clinical Orthopaedics and Related Research 2015;473(10):3235-41.

Ware 1996

Ware JE, Kosinski N, Keller SD. The 12-Item ShortForm Health Survey (SF-12): construction of scales and preliminary tests of reliability and validity. Medical Care 1996;34(3):220-33.

\section{References to other published versions of this review}

\section{Handoll 2002}

Handoll HHG, Madhok R, Howe TE. Rehabilitation for distal radial fractures in adults. Cochrane Database of Systematic Reviews 2002, Issue 2.

\section{Handoll 2003d}

Handoll HHG, Madhok R, Howe TE. Rehabilitation for distal radial fractures in adults. Cochrane Database of Systematic Reviews 2003, Issue 2.

\section{Handoll 2004a}

Handoll HHG, Madhok R, Howe TE. Rehabilitation for distal radial fractures in adults. Cochrane Database of Systematic Reviews 2004, Issue 4.

\section{Handoll 2004b}

Handoll HHG, Madhok R, Howe TE. Rehabilitation for distal radial fractures in adults. Cochrane Database of Systematic Reviews 2004, Issue 4.

\section{Handoll 2006}

Handoll HHG, Madhok R, Howe TE. Rehabilitation for distal radial fractures in adults. Cochrane Database of Systematic Reviews 2006, Issue 3. [DOI: 10.1002/ 14651858.CD003324.pub2]

* Indicates the major publication for the study 


\section{CHARACTERISTICS OF STUDIES}

\section{Characteristics of included studies [ordered by study ID]}

Bache 2001

$\begin{array}{ll}\text { Methods } & \text { Method of randomisation: sealed envelopes contained in a box } \\ \text { Assessor blinding: yes for objective measures; some participants revealed their treatment } \\ \text { despite requests not to do so beforehand } \\ \text { Intention-to-treat analysis: likely, but post-randomisation exclusions: } 4 \text { developed CRPS- } \\ 1 \text { and } 1 \text { developed carpal tunnel syndrome } \\ \text { Loss to follow-up: } 18 \text { (+ above } 5 \text { exclusions) (at } 12 \text { weeks) }\end{array}$

Interventions

Timing of intervention: following plaster cast removal ( 5 to 6 weeks immobilisation). All participants given explanation of home care based on standardised advice and exercise sheet by physiotherapist

1. Referral for routine physiotherapy at outpatients clinic. Contents of treatment at discretion of physiotherapists; these involved different combinations of physiological mobilisation, progressive active exercise, passive stretching, accessory movements of wrist and radioulnar joints. Discharge criteria: functional ROM, full function, plateau of improvement

2. Home exercises alone

Outcomes

Length of follow-up: (median) 12 weeks; also (median) 4 weeks.

1. Functional: grip strength, ROM (pronation; supination; flexion; extension; radial deviation; ulnar deviation); functional analysis scale (Levine 1993), pain (VAS)

Referral to occupational therapy

2. Number of contacts with physiotherapist; duration of physiotherapy, reasons for discharge

3. Complications: carpal tunnel syndrome \& CRPS-1 (mainly excluded from followup)

Funding, ethics and patient consent

No mention of funding or ethics

Informed patient consent

Rehabilitation for distal radial fractures in adults (Review) 
Bache 2001 (Continued)

Notes

Draft trial report received from Mrs Sarah Bache, now based in Australia, on 30 August 2001, and further details on 5 September. Further discussion on outcome measures on 12 September with feedback from trial statistician Louise Hiller

\section{Risk of bias}

\begin{tabular}{|c|c|c|}
\hline Bias & Authors' judgement & Support for judgement \\
\hline $\begin{array}{l}\text { Random sequence generation (selection } \\
\text { bias) }\end{array}$ & Low risk & "Randomised" but statistician involved \\
\hline Allocation concealment (selection bias) & Unclear risk & $\begin{array}{l}\text { Draft report: "Consented patients were } \\
\text { randomised individually by staff with } \\
\text { sealed envelopes contained in a box". How- } \\
\text { ever, the trial investigator confirmed that } \\
\text { there was "a possible chance of disclosure } \\
\text { of assignment" }\end{array}$ \\
\hline
\end{tabular}

Blinding of participants and personnel High risk (performance bias)

Neither participants nor personnel providing care were blinded

Subjective outcomes

Blinding of participants and personnel High risk (performance bias)

Neither participants nor personnel provid-

Objective outcomes

ing care were blinded

Blinding of outcome assessment (detection Unclear risk

bias)

Subjective outcomes

Abstract: "Blind assessment were performed at baseline (within one week of cast removal), and at four and 12 weeks" Draft report: "some patients discussed their treatment at review even though it was stated in the patient information sheet that this should not be done"

Blinding of outcome assessment (detection Unclear risk

As above

bias)

Objective outcomes (functional impair-

ment)

Blinding of outcome assessment (detection Low risk

bias)

Complications, number of sessions, return

to former activity

Incomplete outcome data (attrition bias) Unclear risk

Short term follow-up (up to 3 months)

Outcomes unlikely to be affected by lack of blinding

There were 5 post-randomisation exclusions and imbalance in the numbers lost to follow-up ( $16 \%$ versus $27 \%$ at 12 weeks)

Rehabilitation for distal radial fractures in adults (Review) 
Bache 2001 (Continued)

\begin{tabular}{l|l|l}
\hline $\begin{array}{l}\text { Incomplete outcome data (attrition bias) } \\
\text { Longer term follow-up }\end{array}$ & Unclear risk & No longer-term follow-up \\
\hline Selective reporting (reporting bias) & Unclear risk & $\begin{array}{l}\text { No protocol available. However, consisten- } \\
\text { cies noted with draft report and trial reg- } \\
\text { istration document. Area under the curve } \\
\text { analyses in response to detected baseline } \\
\text { differences }\end{array}$ \\
\hline Major baseline imbalance bias? & Unclear risk & $\begin{array}{l}\text { Some trends towards patients randomised } \\
\text { to physiotherapy and advice and exercise } \\
\text { being more symptomatic at baseline. (Ad- } \\
\text { justments made in analyses) }\end{array}$ \\
\hline $\begin{array}{l}\text { Other performance bias (e.g. differential } \\
\text { expertise bias) }\end{array}$ & Low risk & $\begin{array}{l}\text { No problems detected } \\
\text { None of the control group patients received } \\
\text { physiotherapy or occupational therapy }\end{array}$ \\
\hline
\end{tabular}

Basso 1998

\begin{tabular}{|c|c|}
\hline Methods & $\begin{array}{l}\text { Quasi-randomised: by year of birth } \\
\text { Assessor blinding: yes } \\
\text { Intention-to-treat analysis: likely } \\
\text { Loss to follow-up: none probably }\end{array}$ \\
\hline Participants & $\begin{array}{l}\text { Edgeware General Hospital, UK } \\
38 \text { participants } \\
\text { Inclusion criteria: Colles' fracture, manipulated and treated with plaster cast. Complete } \\
\text { transverse extra-articular break with minimal degree of dorsal displacement and com- } \\
\text { minution } \\
\text { Exclusion criteria: (often by example of the } 13 \text { excluded participants) age }<15 \text { years, intra- } \\
\text { articular involvement, palmar/no displacement, severe dorsal comminution, damage } \\
\text { to ulnar styloid, severe disruption of DRUJ ( }>25 \text { degrees dorsal displacement or }>6 \\
\text { mm radial shortening) and triangular fibrocartilage, carpal injury, inadequate reduction, } \\
\text { more than one manipulation, open fracture, multiple trauma, history of injury to the } \\
\text { contralateral wrist, inability to cope with measuring technique, very poor hand function } \\
\text { following POP removal } \\
\text { Classification: none } \\
\text { Sex: } 22 \text { female ( } 58 \% \text { ) } \\
\text { Age: median } 57 \text { years [ultrasound] and } 63 \text { years [control]; range } 15 \text { to } 69 \text { years } \\
\text { Assigned: } 19 / 19 \text { [ultrasound / control] } \\
\text { Assessed: } 19 / 19 \text { (at } 8 \text { weeks) }\end{array}$ \\
\hline Interventions & $\begin{array}{l}\text { Timing of intervention: following plaster cast removal (on average after } 4 \text { weeks immo- } \\
\text { bilisation; range } 3 \text { to } 8 \text { weeks) } \\
\text { All participants were given instructions to use hands as much as possible. No physio- } \\
\text { therapy "unless hand function was poor" } \\
\text { 1. Ultrasound: } 46.39 \mathrm{kHz} \text { at intensity } 74 \mathrm{~W} / \mathrm{cm} 2 \text { applied for } 5 \text { minutes to back of wrist. }\end{array}$ \\
\hline
\end{tabular}

Rehabilitation for distal radial fractures in adults (Review) 
Joint actively mobilised during treatment

2. Sham ultrasound. Joint actively mobilised for 5 minutes but machine not active (generator still switched on)

Outcomes

Length of follow-up: 8 weeks; also 2 weeks and to end of treatment for those prescribed physiotherapy after 8 weeks

1. Functional: ROM (extension-flexion) loss

2. Referral for physiotherapy at 8 weeks, length of follow-up

Funding, ethics and patient consent

No mention of funding or ethics. (The equipment was provided already calibrated, and reviewed routinely by the manufacturer")

All participants gave informed consent

$\begin{array}{lll}\text { Notes } & \text { Request for further information sent } 8 \text { August } 2001\end{array}$

\section{Risk of bias}

\begin{tabular}{|c|c|c|}
\hline Bias & Authors' judgement & Support for judgement \\
\hline $\begin{array}{l}\text { Random sequence generation (selection } \\
\text { bias) }\end{array}$ & High risk & $\begin{array}{l}\text { "Patients were randomly allocated by year- } \\
\text { of-birth.." }\end{array}$ \\
\hline Allocation concealment (selection bias) & High risk & $\begin{array}{l}\text { "Patients were randomly allocated by year- } \\
\text { of-birth.." }\end{array}$ \\
\hline $\begin{array}{l}\text { Blinding of participants and personnel } \\
\text { (performance bias) } \\
\text { Subjective outcomes }\end{array}$ & Unclear risk & No subjective outcomes \\
\hline $\begin{array}{l}\text { Blinding of participants and personnel } \\
\text { (performance bias) } \\
\text { Objective outcomes }\end{array}$ & Low risk & $\begin{array}{l}\text { "In the control group, the procedure was } \\
\text { the same as in the treatment group but the } \\
\text { generator, although switched on, was not } \\
\text { active" }\end{array}$ \\
\hline
\end{tabular}

Blinding of outcome assessment (detection Unclear risk

No subjective outcomes

bias)

Subjective outcomes

Blinding of outcome assessment (detection Low risk bias)

Objective outcomes (functional impairment)

Blinding of outcome assessment (detection Low risk bias)

Complications, number of sessions, return to former activity
"When reviewing a patient at 8 weeks, the observer (OB) had no access to the records and was unaware of the treatment given until after assessment of hand function and pain"

Outcomes unlikely to be affected by lack of blinding 


\begin{tabular}{|c|c|c|}
\hline $\begin{array}{l}\text { Incomplete outcome data (attrition bias) } \\
\text { Short term follow-up (up to } 3 \text { months) }\end{array}$ & Unclear risk & $\begin{array}{l}\text { There appears to be no loss to follow-up. } \\
\text { However, it is stated that "It was considered } \\
\text { ethically unacceptable to retain patients in } \\
\text { the study who had very poor hand function } \\
\text { following removal of the plaster" }\end{array}$ \\
\hline $\begin{array}{l}\text { Incomplete outcome data (attrition bias) } \\
\text { Longer term follow-up }\end{array}$ & Unclear risk & No longer-term follow-up \\
\hline Selective reporting (reporting bias) & Unclear risk & No protocol available \\
\hline Major baseline imbalance bias? & Unclear risk & $\begin{array}{l}\text { Incomplete information including on du- } \\
\text { ration of prior immobilisation }\end{array}$ \\
\hline $\begin{array}{l}\text { Other performance bias (e.g. differential } \\
\text { expertise bias) }\end{array}$ & Unclear risk & $\begin{array}{l}\text { Incomplete information on care pro- } \\
\text { grammes. Possible provision of physiother- } \\
\text { apy for poor hand function }\end{array}$ \\
\hline
\end{tabular}

Bighea 2013

Methods

$\begin{array}{ll}\text { Methods } & \text { Randomised: no details of method } \\ & \text { Assessor blinding: no report (unlikely) } \\ \text { Intention-to-treat analysis: not known } \\ \text { Loss to follow-up: none probably }\end{array}$

Participants
University of Medicine and Pharmacy of Craiova, Craiova, Romania

20 participants

Inclusion criteria: osteoporotic wrist fracture

Exclusion criteria: No details

Classification: none

Sex: not reported

Age: not reported

Assigned: 10/10 [physiotherapy / control]

Assessed: ?/? (at 8 weeks?; 4 weeks post-immobilisation )
Interventions

Outcomes
Timing of intervention: probably following plaster cast removal (after 4 weeks immobilisation)

1. Physiotherapy: 20 minutes galvanic bath then 30 minutes exercise programme performed 5 times per week, once daily, involving active range of motion, hand and finger flexion and extension, and ball resistance. Four weeks of therapy before second examination reported

2. Home exercise programme. Detailed instructions given to follow a 15 minutes twice a day home exercise programme

Length of follow-up: 8 weeks (timing of randomisation not stated); first examination just after 4 weeks immobilisation; second examination after 4 weeks of rehabilitative treatment 
Bighea 2013 (Continued)

1. Functional: PRWE, ROM (extension, flexion)

\begin{tabular}{l|l}
\hline Funding, ethics and patient consent & $\begin{array}{l}\text { No mention of funding or ethics } \\
\text { No mention of informed consent }\end{array}$ \\
\hline Notes & Request for further information sent 4 May 2015 \\
\hline
\end{tabular}

Risk of bias

\begin{tabular}{|c|c|c|}
\hline Bias & Authors' judgement & Support for judgement \\
\hline $\begin{array}{l}\text { Random sequence generation (selection } \\
\text { bias) }\end{array}$ & Unclear risk & "randomly assigned into two groups" \\
\hline Allocation concealment (selection bias) & Unclear risk & "randomly assigned into two groups" \\
\hline $\begin{array}{l}\text { Blinding of participants and personnel } \\
\text { (performance bias) } \\
\text { Subjective outcomes }\end{array}$ & High risk & Not blinded \\
\hline $\begin{array}{l}\text { Blinding of participants and personnel } \\
\text { (performance bias) } \\
\text { Objective outcomes }\end{array}$ & High risk & Not blinded \\
\hline
\end{tabular}

\begin{tabular}{l|l} 
Blinding of outcome assessment (detection High risk Not blinded
\end{tabular}

bias)

Subjective outcomes

$\begin{aligned} & \text { Blinding of outcome assessment (detection } \\ & \text { bias) }\end{aligned}$
$\begin{aligned} & \text { Objective outcomes (functional impair- } \\ & \text { ment) }\end{aligned}$

Blinding of outcome assessment (detection Unclear risk

Outcomes not reported bias)

Complications, number of sessions, return

to former activity

$\begin{array}{lll}\text { Incomplete outcome data (attrition bias) } & \text { Unclear risk } \quad \text { No report of participant flow }\end{array}$

Short term follow-up (up to 3 months)

Incomplete outcome data (attrition bias) Unclear risk

Longer term follow-up

\begin{tabular}{|c|c|c|}
\hline Selective reporting (reporting bias) & High risk & $\begin{array}{l}\text { No protocol. Conference abstract only. } \\
\text { Minimal data }\end{array}$ \\
\hline Major baseline imbalance bias? & Unclear risk & Balance claimed but no supporting data \\
\hline
\end{tabular}

Rehabilitation for distal radial fractures in adults (Review) 
Bighea 2013 (Continued)

Other performance bias (e.g. differential Unclear risk

No information expertise bias)

Brehmer 2014

Methods

Method of randomisation: use of computerised random number generation; administered by an independent research co-ordinator

Assessor blinding: surgeons were but not assessors, including hand therapists

Intention-to-treat analysis: no, 3 exclusions in the standard rehabilitation group

Loss to follow-up: 15 (at 6 months)

Participants

TRIA Orthopaedic Center, University of Minnesota, Bloomington, Minnesota, USA

81 participants

Inclusion criteria: age 18 to 85 years; isolated distal radial fracture treated with open reduction and internal fixation using a plate (Hand Innovations DVR plate) and informed consent.

Exclusion criteria: previous distal radial fracture on the affected side; professional athlete; bilateral distal radial fracture; another concurrent fracture; and a distal radial fracture for which, in the surgeon's judgment, fixation of fracture fragments could not be achieved to allow participation in the accelerated rehabilitation protocol.

Classification: AO fracture classification

Sex (of 78): female (73\%)

Age: mean 53 years (range 21 to 83 years)

Assigned: 36/45 [accelerated / standard]

Assessed: 33/30 (at 6 months)

Interventions

Timing of intervention: both groups began with gentle active range of motion at three to five days after plate fixation using the same type of plate. Custom splint applied (30 degrees wrist extension) removed for hygiene, dressing, eating, exercises. Scar management began when sutures were removed in both groups

1. Accelerated rehabilitation group. Patients initiated wrist/forearm passive range of motion and strengthening exercises at two weeks. Weaned from splint at week 3, discontinued splint at week 4 . Isotonic and medium putty strengthening at week 4 ; heavy putty strengthening at week 6

2. 'Standard' rehabilitation group. Patients initiated wrist/forearm passive range of motion and strengthening exercises at six weeks. Weaned from splint at week 6, discontinued splint at week 7. Isotonic and medium putty strengthening at week 7; heavy putty strengthening at week 8

Outcomes

Length of follow-up: 6 months post-operatively; also 2, 3, 4, 6, 8 and 12 weeks 1. Functional: Disabilities of the Arm, Shoulder and Hand (DASH) scores; grip strength, palmar pinch strength; ROM (wrist flexion, extension, supination, pronation);

2. Clinical: adverse events and splint repair

3. Others: fracture alignment and healing

Funding, ethics and patient consent

Study received "limited financial support (from a research grant) from" a commercial company but no implants were involved. Approval reported by institutional review board Informed consent was obtained from all participants

Rehabilitation for distal radial fractures in adults (Review)

Copyright @ 2015 The Cochrane Collaboration. Published by John Wiley \& Sons, Ltd. 
Brehmer 2014 (Continued)

Notes

Risk of bias

\begin{tabular}{lll} 
Bias & Authors' judgement & Support for judgement \\
\hline $\begin{array}{l}\text { Random sequence generation (selection } \\
\text { bias) }\end{array}$ & Low risk & $\begin{array}{l}\text { "Randomization was performed by com- } \\
\text { puterized random number generation" }\end{array}$ \\
\hline Allocation concealment (selection bias) & Low risk & $\begin{array}{l}\text { Although no details, there was blinding and } \\
\text { independent randomisation: "Enrollment } \\
\text { was performed by a research coordinator } \\
\text { who was blinded to fracture severity and, if } \\
\text { consent had been obtained postoperatively } \\
\text { but before initiation of therapy, to the op- } \\
\text { erative result" }\end{array}$
\end{tabular}

Blinding of participants and personnel High risk (performance bias)

"All participating surgeons were blinded to each patient's randomization group as the surgeons' postoperative care was the same for the two groups. Due to the nature of the study, the hand therapists were not blinded.

" Participants were also not blinded

Blinding of participants and personnel High risk

As above

(performance bias)

Objective outcomes

Blinding of outcome assessment (detection High risk

Participants were not blinded

bias)

Subjective outcomes

Blinding of outcome assessment (detection High risk

bias)

Objective outcomes (functional impair-

ment)

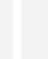


Brehmer 2014 (Continued)

\begin{tabular}{|c|c|c|}
\hline $\begin{array}{l}\text { Incomplete outcome data (attrition bias) } \\
\text { Longer term follow-up }\end{array}$ & High risk & $\begin{array}{l}\text { Over three times as many loses in the usual } \\
\text { group at } 6 \text { months: } 3 / 36(8.3 \%) \text { versus } 12 / \\
45(26.7 \%)\end{array}$ \\
\hline
\end{tabular}

\begin{tabular}{l|l|l} 
Selective reporting (reporting bias) & Low risk & $\begin{array}{l}\text { No protocol available nor trial registra- } \\
\text { tion but assessment was straightforward, } \\
\text { systematic and covered sufficient key out- } \\
\text { comes }\end{array}$ \\
\hline Major baseline imbalance bias? & Unclear risk & $\begin{array}{l}\text { Some imbalance in fracture distribution of } \\
\text { unknown importance. Accelerated group 5 } \\
\text { years younger on average }\end{array}$
\end{tabular}

Other performance bias (e.g. differential Low risk expertise bias)

Since the same programme was applied but at different times the expertise of the hand therapists would have mattered less

Challis 2007

Methods

Method of randomisation: opaque envelopes contained in a box

Assessor blinding: yes for objective measures.

Intention-to-treat analysis: yes

Loss to follow-up: 2 (at 6 and 10 weeks)

Participants

General Hospital, Queensland, Australia

21 participants

Inclusion criteria: distal radius fracture, treated by plaster cast immobilisation, age 18 years or above, informed consent.

Exclusion criteria: open skin lesions, nerve or tendon damage associated with the fracture, fracture required surgical fixation or known pathology in their intact arm.

Classification: "Rockwood + Green" (4 categories: extra-articular undisplaced, extraarticular displaced; intra-articular undisplaced; intra-articular displaced): distribution not reported.

Sex: 15 female $(76 \%)$

Age: mean 51 years

Assigned: 11/10 [pneumatic compression / control]

Assessed: 10/9 (at 10 weeks)

Interventions

Timing of intervention: following initial treatment of fracture in a split cast. Allocation was at a mean of 9.4 days after fracture. The split cast was replaced with a full forearm plaster, worn for 5 weeks. All participants had an inflatable cuff positioned around their forearm under their plaster with the valve of the cuff protruding through the cast. Both groups were instructed to actively make a fist 100 times per day during the immobilisation period. All participants had a total of 6 weeks immobilisation.

1. Cyclic pneumatic soft tissue compression for 5 weeks. Participants were provided with a compression pump apparatus to use at home and received instructions on its use. The apparatus consisted of a compression pump connected to an inflatable cuff positioned around the proximal forearm flexor and extensor muscle bulk under the plaster. The

Rehabilitation for distal radial fractures in adults (Review)

Copyright () 2015 The Cochrane Collaboration. Published by John Wiley \& Sons, Ltd. 
compression pump was designed to pump air into a reservoir and, at set time periods, release a pressurised volume of air into the inflatable cuff. One inflation/deflation of the cuff took 10 seconds and 60 compressions were applied per treatment session. The cyclic pneumatic pressure was applied twice per day (morning and evening) taking ten minutes for each session. After piloting on two cases, the pressurising period of 3.5 seconds was chosen on the basis that it did not produce any pain at the fracture site

2. 'Usual care'. (See above)

After plaster cast removal, all participants were given a programme of strengthening and stretching exercises for the hand, wrist, and forearm to be carried out twice a day for four weeks

Outcomes

Length of follow-up: 10 weeks; also 6 weeks

1. Functional: strength (power grip, pinch grip, key grip, supination (isometric), ROM (pronation/ supination; flexion/extension). Absolute and \% 'intact side'

Funding, ethics and patient consent

No mention of funding. Ethical approval from hospital ethics committee Informed patient consent

Notes

Individual patient data provided in an appendix for 'absolute' outcomes reveal that the standard deviations were probably standard errors. Results presented here are derived from individual patient data and thus the results relative to the uninjured side are not presented in this review given the concerns about data discrepancies

Risk of bias

Bias

Authors' judgement

Support for judgement

Random sequence generation (selection Low risk bias)

"Participants were then randomly allocated to either the experimental group or the control group by the fracture clinic nurse, who drew opaque envelopes containing the concealed group allocation from a box"

Allocation concealment (selection bias) Low risk

"Participants were then randomly allocated to either the experimental group or the control group by the fracture clinic nurse, who drew opaque envelopes containing the concealed group allocation from a box"

Blinding of participants and personnel Unclear risk

No subjective outcomes measured (performance bias)

Subjective outcomes

Blinding of participants and personnel High risk

Participants and personnel not blinded (performance bias)

Objective outcomes

Rehabilitation for distal radial fractures in adults (Review) 
Challis 2007 (Continued)

\begin{tabular}{|c|c|c|}
\hline $\begin{array}{l}\text { Blinding of outcome assessment (detection } \\
\text { bias) } \\
\text { Subjective outcomes }\end{array}$ & Unclear risk & No subjective outcomes measured \\
\hline $\begin{array}{l}\text { Blinding of outcome assessment (detection } \\
\text { bias) } \\
\text { Objective outcomes (functional impair- } \\
\text { ment) }\end{array}$ & Low risk & $\begin{array}{l}\text { "All participants had an inflatable cuff po- } \\
\text { sitioned around their forearm under their } \\
\text { plaster with the valve of the cuff protruding } \\
\text { through the cast so that the independent } \\
\text { assessor remained blind to group allocation } \\
\text { during all measurements" }\end{array}$ \\
\hline
\end{tabular}

Blinding of outcome assessment (detection Unclear risk

Outcomes not recorded

bias)

Complications, number of sessions, return

to former activity

Incomplete outcome data (attrition bias) Unclear risk

Short term follow-up (up to 3 months)

"Two participants were lost to follow-up: one participant (experimental group) removed the plaster prematurely and could not receive the intervention, and the other (control group) failed to attend measurement sessions and withdrew within the first three weeks of the trial"

Incomplete outcome data (attrition bias) Unclear risk $\quad$ No longer-term follow-up

Longer term follow-up

\begin{tabular}{lll}
\hline Selective reporting (reporting bias) $\quad$ Unclear risk & $\begin{array}{l}\text { No protocol and no trial registration avail- } \\
\text { able }\end{array}$
\end{tabular}

Major baseline imbalance bias?

High risk

The populations in the two groups differed importantly: intervention group 5/11 were male, mean age 46 years, and "more severe fractures"; control group $0 / 10$ were male, mean age 57 years

Other performance bias (e.g. differential Low risk expertise bias)

Same treatment other than intervention applied to both groups

Cheing 2005

Methods

Method of randomisation: by drawing lots (non-replacement method)

Assessor blinding: no

Intention-to-treat analysis: likely

Loss to follow-up: none

Rehabilitation for distal radial fractures in adults (Review) 
Cheing 2005 (Continued)

\begin{tabular}{l} 
Queen Elizabeth Hospital, Hong Kong \\
Participants \\
Inclusion criteria: "stable'“ distal radial fracture treated by closed reduction and 6 weeks \\
plaster cast immobilisation. Informed consent. Able to communicate independently \\
Exclusion criteria: CRPS-1, inflammatory arthritis, perivascular disease, previous fracture \\
or neurovascular injuries in the affected hand, heart disease, use of heart pacemaker or \\
other auxiliary organs, tuberculosis, viral infections, juvenile diabetes, mycosis, internal \\
haemorrhages, or pregnancy. Recently had deep X-Ray therapy or pulsed electromagnetic \\
treatment during immobilisation period \\
Classification: None given \\
Sex: 55 female $(66 \%)$ \\
Age: mean 63 years; range 17 to 80 years \\
Assigned: $23 / 22 / 22 / 16$ [PEMF+ice/ sham PEMF+ice/ PEMF / sham PEMF] \\
Assessed: $23 / 22 / 22 / 16$ (at 5 days) \\
\hline
\end{tabular}

Interventions

Timing of intervention: 3 to 4 days following plaster cast removal (6 weeks immobilisation)

All treatments were 30 minutes for 5 consecutive days. After the first treatment, the participants were taught and given written instructions for a home exercise programme of active wrist and finger mobilisation exercises and advised to do these twice a day for 20 minutes each session. Exercise compliance was checked by the physiotherapist at each treatment session

1. Pulsed electromagnetic field (PEMF) at $50 \mathrm{~Hz}$ with a field intensity of 99 gauss, and ice (1 kg pack of flaked ice wrapped in towel and placed dorsally)

2. Sham PEMF and ice

3. PEMF

4. Sham PEMF

Outcomes

Length of follow-up: 4 days (before the 5th treatment session); also 2 days (before 3rd session)

1. Functional: pain (VAS during mobilisation), ROM (pronation; supination; flexion; extension; radial deviation; ulnar deviation)

2. Clinical: oedema. Adverse events ("none reported")

Funding, ethics and patient consent

Notes
No mention of funding or ethics. Informed consent obtained

Reply received from A/Prof Cheing on 9 December 2005 who provided further details of the methods, including randomisation, and also stated there were no adverse events reported

\section{Risk of bias}

\begin{tabular}{|c|c|c|}
\hline Bias & Authors' judgement & Support for judgement \\
\hline $\begin{array}{l}\text { Random sequence generation (selection } \\
\text { bias) }\end{array}$ & Unclear risk & $\begin{array}{l}\text { Email }(09 / 12 / 2005) \text { : "Group allocation } \\
\text { was done by drawing lots (non-replacement } \\
\text { method)“ }\end{array}$ \\
\hline
\end{tabular}

Rehabilitation for distal radial fractures in adults (Review) 


\section{Cheing 2005 (Continued)}

\begin{tabular}{|c|c|c|}
\hline Allocation concealment (selection bias) & Unclear risk & $\begin{array}{l}\text { Email }(09 / 12 / 2005) \text { : "Group allocation } \\
\text { was done by drawing lots (non-replacement } \\
\text { method).“. No information to judge }\end{array}$ \\
\hline $\begin{array}{l}\text { Blinding of participants and personnel } \\
\text { (performance bias) } \\
\text { Subjective outcomes }\end{array}$ & Unclear risk & $\begin{array}{l}\text { "participants were blinded as to whether } \\
\text { they received PEMF or sham PEMF." Per- } \\
\text { sonnel may have been aware of circuit being } \\
\text { disconnected at the back of the machine." } \\
\text { No blinding for ice intervention }\end{array}$ \\
\hline $\begin{array}{l}\text { Blinding of participants and personnel } \\
\text { (performance bias) } \\
\text { Objective outcomes }\end{array}$ & Unclear risk & As above \\
\hline $\begin{array}{l}\text { Blinding of outcome assessment (detection } \\
\text { bias) } \\
\text { Subjective outcomes }\end{array}$ & Unclear risk & $\begin{array}{l}\text { No mention of blinding of outcome assess- } \\
\text { ment, but follow-up assessment within the } \\
\text { treatment period }\end{array}$ \\
\hline $\begin{array}{l}\text { Blinding of outcome assessment (detection } \\
\text { bias) } \\
\text { Objective outcomes (functional impair- } \\
\text { ment) }\end{array}$ & Unclear risk & $\begin{array}{l}\text { No mention of blinding of outcome assess- } \\
\text { ment, but follow-up assessment within the } \\
\text { treatment period }\end{array}$ \\
\hline $\begin{array}{l}\text { Blinding of outcome assessment (detection } \\
\text { bias) } \\
\text { Complications, number of sessions, return } \\
\text { to former activity }\end{array}$ & Low risk & $\begin{array}{l}\text { Outcomes unlikely to be affected by lack } \\
\text { of blinding }\end{array}$ \\
\hline $\begin{array}{l}\text { Incomplete outcome data (attrition bias) } \\
\text { Short term follow-up (up to } 3 \text { months) }\end{array}$ & Unclear risk & $\begin{array}{l}\text { No lost to follow-up but trial failed to } \\
\text { record outcome after the end of treatment }\end{array}$ \\
\hline $\begin{array}{l}\text { Incomplete outcome data (attrition bias) } \\
\text { Longer term follow-up }\end{array}$ & Unclear risk & No longer-term follow-up \\
\hline Selective reporting (reporting bias) & Unclear risk & Protocol not available \\
\hline Major baseline imbalance bias? & Unclear risk & $\begin{array}{l}\text { Baseline imbalances in some variables, such } \\
\text { as extension }\end{array}$ \\
\hline $\begin{array}{l}\text { Other performance bias (e.g. differential } \\
\text { expertise bias) }\end{array}$ & Low risk & No problems detected \\
\hline
\end{tabular}


Methods

Method of randomisation: use of sealed envelopes (concealment confirmed by trialist) Assessor blinding: yes

Intention-to-treat analysis: likely but for 2 excluded from analyses ( 1 death and 1 with severe pain after cast removal)

Loss to follow-up: none (except 2 exclusions)
Interventions

Outcomes

Funding, ethics and patient consent

Notes
University Hospital Gentofte, Denmark

32 participants

Inclusion criteria: Colles' fracture, treated with plaster cast.

Exclusion criteria: none provided.

Classification: Older's classification

Sex: (of 30) 27 female (90\%)

Age: (of 30) mean 66 years; range 46 to 82 years

Assigned: 16/16 [occupational therapy / control]

Assessed: 16/14 (at 9 months)

Timing of intervention: following plaster cast removal (5 weeks immobilisation)

All participants were given instructions by occupational therapist for shoulder, wrist and fingers exercises to be performed thrice daily at home

1. Occupational therapy involving active joint exercises for wrist, elbow and shoulder; oedema prevention; coordination exercise; coarse and fine motor-function exercise; strengthening exercise; sensation exercise; ADL training. "Distributed" around twice weekly sessions until therapist considered no further progress was being made.

2. Home exercises only

Length of follow-up: 9 months; also 3 months

1. Functional: grip strength, Solgaard modified Gartland and Werley score

2. Number of sessions and overall duration of occupational therapy

No mention of funding

No mention of patient consent. Mention of the approval of protocol by local ethics committee

Replies received 20 and 21 August 2001

Risk of bias

\begin{tabular}{|c|c|c|}
\hline Bias & Authors' judgement & Support for judgement \\
\hline $\begin{array}{l}\text { Random sequence generation (selection } \\
\text { bias) }\end{array}$ & Unclear risk & $\begin{array}{l}\text { "Randomized trial." No information on se- } \\
\text { quence generation }\end{array}$ \\
\hline Allocation concealment (selection bias) & Low risk & $\begin{array}{l}\text { "The allocation principle was by the closed } \\
\text { envelope method" } \\
\text { Letter dated 19/08/2001: "The envelopes } \\
\text { were sealed and allocation was concealed at } \\
\text { the time of randomisation" }\end{array}$ \\
\hline
\end{tabular}




\section{Christensen 2001 (Continued)}

Blinding of participants and personnel Unclear risk (performance bias)

Subjective outcomes
No subjective outcomes except within Gartland and Werley score

Neither participants nor personnel providing care were blinded
High risk (performance bias)

Objective outcomes
No subjective outcomes except within Gartland and Werley score
Blinding of outcome assessment (detection Unclear risk bias)

Subjective outcomes
Blinding of outcome assessment (detection Low risk bias)

Objective outcomes (functional impairment)
Letter dated 19/08/2001: "The outcome assessments were made by myself and $\mathrm{T}$. C. Christiansen. At the time of the assessments we did not know to which group the patients were allocated"

Outcomes unlikely to be affected by lack of blinding
Blinding of outcome assessment (detection Low risk bias)

Complications, number of sessions, return

to former activity

Incomplete outcome data (attrition bias) Unclear risk Short term follow-up (up to 3 months)
Letter dated 19/08/2001: "No patients were lost to follow up. Two patients were excluded from the study. One patient died after the 3 months follow up and the other had severe pain at cast removal - had further immobilisation and developed reflex sympathetic dystrophy." Further email (21/ 08/2001) explained that both patients were in the "non-occupational group." Incomplete data provided on Gartland and Werley scores. (However, grip strength data provided)

Incomplete outcome data (attrition bias) Unclear risk As above

Longer term follow-up

\begin{tabular}{lll}
\hline Selective reporting (reporting bias) & Unclear risk & No protocol available \\
\hline Major baseline imbalance bias? & Low risk & No major imbalance \\
\hline $\begin{array}{l}\text { Other performance bias (e.g. differential } \\
\text { expertise bias) }\end{array}$ & Low risk & $\begin{array}{l}\text { No problems detected. } \\
\text { Letter dated 19/08/2001: "Both groups re- } \\
\text { ceived instruction by occupational thera- } \\
\text { pist" } \\
\text { "No patients in the non-occupational }\end{array}$
\end{tabular}

Rehabilitation for distal radial fractures in adults (Review) 
Christensen 2001 (Continued)

group received occupational therapy"

\section{Cooper 2001}

Methods

Method of randomisation: independent person generated sealed numbered opaque envelopes using a random numbers table - researcher had no knowledge of allocation in advance

Assessor blinding: no

Intention-to-treat analysis: yes

Loss to follow-up: none

Participants

Pilgrim Hospital, Boston, UK

17 participants

Inclusion criteria: Distal radial fracture treated conservatively with closed reduction and immobilisation, age $>16$ years (adult), willing and able to attend the department for assessment and treatment. informed consent

Exclusion criteria: frail elderly people with mobility problems preventing attendance, impaired mental or cognitive ability, multiple fractures or extensive soft tissue injuries, surgical treatment or pre-morbid neurological conditions

Classification: None given

Sex: 16 female $(94 \%)$

Age: mean 65.5 years; range 41 to 81 years

Assigned: 8/9 [early intervention/control]

Assessed: 8/9 (at 4 weeks post-removal of plaster cast)

Interventions

Timing of intervention: within 4 days of fracture (routinely 4 weeks immobilisation, or, for some, 6 weeks)

All participants received home treatment programme including written advice about skin care, control of oedema, wrist and forearm exercises at fracture clinic and cast application. Post-immobilisation care programme for all participants comprised an individualised home programme and, where prespecified criteria were met, attendance of a hand therapy group

1. "Early therapeutic intervention" with oedema management, active range of movement of uninvolved joints (fingers, elbow, shoulder and neck), monitoring of plaster cast, written information and contact number of project team. Weekly contact with member of hand therapy team

2. "Standard intervention" only, started after plaster cast removal

Outcomes

Length of follow-up: 4 weeks post-immobilisation; also 4 days

1. Functional: grip strength, ROM (pronation; supination; flexion; extension; radial deviation; ulnar deviation), functional dexterity (9-hole peg test), pain at rest or during activity (VAS), DASH functional scores, finger movement (total active movement), opposition of thumb (Kapandji scores), pinch grip, and referral to hand therapy

2. Clinical: oedema. Complications: CRPS-1

Funding, ethics and patient consent

No mention of funding or ethics

Written patient consent

Rehabilitation for distal radial fractures in adults (Review)

Copyright @ 2015 The Cochrane Collaboration. Published by John Wiley \& Sons, Ltd. 


\section{Cooper 2001 (Continued)}

Notes

Trial was part of a masters degree in hand therapy

Risk of bias

\begin{tabular}{|c|c|c|}
\hline Bias & Authors' judgement & Support for judgement \\
\hline $\begin{array}{l}\text { Random sequence generation (selection } \\
\text { bias) }\end{array}$ & Low risk & "A table of random numbers was used" \\
\hline Allocation concealment (selection bias) & Low risk & $\begin{array}{l}\text { "The allocation was then placed into en- } \\
\text { velopes and sealed with the subject number } \\
\text { on the outside. ... When allocating patients } \\
\text { to the treatment groups the envelopes were } \\
\text { opened in front of the patients if present, } \\
\text { or a member of staff if carried our over the } \\
\text { telephone, again in order to prevent bias." } \\
\text { Trialist confirmed envelopes were "opaque" }\end{array}$ \\
\hline $\begin{array}{l}\text { Blinding of participants and personnel } \\
\text { (performance bias) } \\
\text { Subjective outcomes }\end{array}$ & High risk & $\begin{array}{l}\text { Neither participants nor personnel provid- } \\
\text { ing care were blinded }\end{array}$ \\
\hline $\begin{array}{l}\text { Blinding of participants and personnel } \\
\text { (performance bias) } \\
\text { Objective outcomes }\end{array}$ & High risk & $\begin{array}{l}\text { Neither participants nor personnel provid- } \\
\text { ing care were blinded }\end{array}$ \\
\hline $\begin{array}{l}\text { Blinding of outcome assessment (detection } \\
\text { bias) } \\
\text { Subjective outcomes }\end{array}$ & High risk & There was no blinding \\
\hline $\begin{array}{l}\text { Blinding of outcome assessment (detection } \\
\text { bias) } \\
\text { Objective outcomes (functional impair- } \\
\text { ment) }\end{array}$ & High risk & There was no blinding \\
\hline
\end{tabular}

Blinding of outcome assessment (detection Low risk bias)

Complications, number of sessions, return

Outcomes unlikely to be affected by lack to former activity

Incomplete outcome data (attrition bias) Low risk

Short term follow-up (up to 3 months)

All participants accounted for. No loss to follow-up

Incomplete outcome data (attrition bias) Unclear risk $\quad$ No longer-term outcomes

Longer term follow-up

Selective reporting (reporting bias) Low risk

No protocol available but comprehensive MSC report indicates no risk 


\section{Cooper 2001 (Continued)}

Major baseline imbalance bias?

High risk
Small number of participants with 9 year difference in the two group's mean age (61 versus 70 )

Other performance bias (e.g. differential Unclear risk

Unknown variation in duration of cast imexpertise bias) mobilisation

Gronlund 1990

Methods Method of randomisation: involved envelopes - stated to be single-blind by trialist Assessor blinding: yes Intention-to-treat analysis: probably Loss to follow-up: probably none

Participants

Fredenksberg Hospital, Alsgarde, Denmark

40 participants

Inclusion criteria: Colles' fracture, unilateral fracture, suitable for plaster cast: stable fracture in plaster, attendance at casualty ward within 24 hours of injury, age $>45$ years, (implied: resident in hospital catchment area)

Exclusion criteria: unstable fracture (reduced position could not be maintained in plaster) , wrist arthritis, other fracture in same limb, neuromuscular pain in limb, dementia or some other condition making participation difficult

Classification: Older

Sex: 35 female (88\%)

Age: median 74.5 years; range 47 to 93 years

Assigned: 17/23 [occupational therapy/control]

Assessed: 17/23 (at 13 weeks)

Interventions

Timing of intervention: following reduction and application of plaster cast. (Approximately 5 weeks immobilisation)

All participants given advice about active movement exercises of shoulder and fingers and information on the problems of plaster casts after application of cast (in casualty)

1. Participant attended rheumatoid disorder outpatients clinic 1 to 3 days after initial treatment. Instructions for hand pumping exercises, active finger, elbow, shoulder movements, assessment of the need for appliances (e.g. angled knives), and for home help provided by occupational therapist. Referral to occupational therapist for rehabilitation if required after plaster cast removal

2. Referral to occupational therapist for rehabilitation if required after plaster cast removal

Outcomes

Length of follow-up: 13 weeks; also 5 and 9 weeks

1. Functional: modified Stewart 1984 (modified Gartland and Werley) functional score (subjective pain, limitations of movement and function; ROM, grip strength, median nerve compression), movement, use of analgesia

2. Clinical: oedema, abnormal sweating, colour, temperature. Complications: CRPS-1, median \& ulnar nerve compression, tendon rupture

3. Use of appliances, home help, plaster cast problems, participant satisfaction, understanding of instructions given at casualty: for intervention group participants only 


\section{Gronlund 1990 (Continued)}

\begin{tabular}{ll}
\hline Funding, ethics and patient consent & $\begin{array}{l}\text { No mention of funding or ethics } \\
\text { All participants gave informed consent }\end{array}$ \\
\hline Notes & $\begin{array}{l}\text { Translation from Danish by Dr Michael Bird } \\
\text { Further details of trial received 20 August 2001. Nine participants previously unac- } \\
\text { counted for had unstable fractures, and were re-admitted, some fractures were fixed with } \\
\text { Hoffman external fixation, and were not included in trial }\end{array}$ \\
\hline
\end{tabular}

\section{Risk of bias}

\begin{tabular}{|c|c|c|}
\hline Bias & Authors' judgement & Support for judgement \\
\hline $\begin{array}{l}\text { Random sequence generation (selection } \\
\text { bias) }\end{array}$ & Low risk & $\begin{array}{l}\text { Completed form } 20 \text { August 2001: "Picking } \\
\text { an envelope with two possibilities (single } \\
\text { blind procedure)" }\end{array}$ \\
\hline
\end{tabular}

Allocation concealment (selection bias) Low risk

Blinding of participants and personnel High risk (performance bias)

Subjective outcomes
In answer to whether allocation was concealed - completed form 20 August 2001: "YES"

Participants and personnel not blinded

Participants and personnel not blinded

Blinding of participants and personnel High risk (performance bias)

Objective outcomes

Blinding of outcome assessment (detection Unclear risk bias)

Subjective outcomes

Blinding of outcome assessment (detection Unclear risk bias)

Objective outcomes (functional impair-

ment)

Blinding of outcome assessment (detection Low risk bias)

Complications, number of sessions, return

to former activity
From translation: "Five, 9, and 13 weeks after the date of the accident, all patients were examined by the same rheumatoid specialist, who did not know to which group the patients belonged." There was, however, no indication of safeguards such as telling the participants not to tell the assessor which group they were in

As above - blinding possible but not mention of safeguards
Outcomes unlikely to be affected by any deficiencies in blinding 


\section{Gronlund 1990 (Continued)}

\begin{tabular}{|c|c|c|}
\hline $\begin{array}{l}\text { Incomplete outcome data (attrition bias) } \\
\text { Short term follow-up (up to } 3 \text { months) }\end{array}$ & Unclear risk & $\begin{array}{l}\text { Some slight unease concerning recruitment } \\
\text { status of } 9 \text { participants, accounted for in } \\
\text { correspondence, that had unstable frac- } \\
\text { tures, and were re-admitted, some fractures } \\
\text { were fixed with Hoffman external fixation, } \\
\text { and were apparently "not included" in the } \\
\text { trial }\end{array}$ \\
\hline $\begin{array}{l}\text { Incomplete outcome data (attrition bias) } \\
\text { Longer term follow-up }\end{array}$ & Unclear risk & No longer-term follow-up \\
\hline Selective reporting (reporting bias) & Unclear risk & No protocol available to judge \\
\hline Major baseline imbalance bias? & Unclear risk & $\begin{array}{l}\text { Probably no major imbalance but there } \\
\text { were differences in the distribution of frac- } \\
\text { ture types reported }\end{array}$ \\
\hline $\begin{array}{l}\text { Other performance bias (e.g. differential } \\
\text { expertise bias) }\end{array}$ & Unclear risk & $\begin{array}{l}\text { Pragmatic trial but lack of information on } \\
\text { occupational therapy referral and mention } \\
\text { of two of the treatment group patients al- } \\
\text { ready having home help arranged by their } \\
\text { general practitioner }\end{array}$ \\
\hline
\end{tabular}

Jongs 2012

Methods

Method of randomisation: off site computerised blocked randomisation and sealed and sequentially numbered envelopes

Assessor blinding: yes at 8 weeks but not at 12 weeks

Intention-to-treat analysis: no, post-randomisation losses, 1 unexplained protocol violation

Loss to follow-up: 8 (at 12 weeks)

Participants

A Sydney metropolitan hospital (Royal North Shore Hospital), Australia

40 participants

Inclusion criteria: distal radius fracture, conservatively or surgically treated patients were referred to physiotherapy by a consultant hand surgeon at least 10 weeks from the time of injury if surgeon was concerned about progress, a stable and united (or uniting) unilateral fracture, wrist contracture evident by a loss of passive extension compared with the unaffected wrist, lived in the Sydney metropolitan region, were willing or likely to co-operate with the intervention, over the age of 18 years and informed consent

Exclusion criteria: patient unlikely to co-operate

Classification: not stated

Sex: female $(70 \%)$

Age: median 66 [dynamic splints] versus 58 years [control]

Assigned: 20/20 [dynamic splints / control] (intention-to-treat analysis); 19/21 (perprotocol analysis)

Assessed: 15/17 (at 12 weeks)(intention-to-treat analysis); 14/18 (per-protocol analysis)

Rehabilitation for distal radial fractures in adults (Review) 


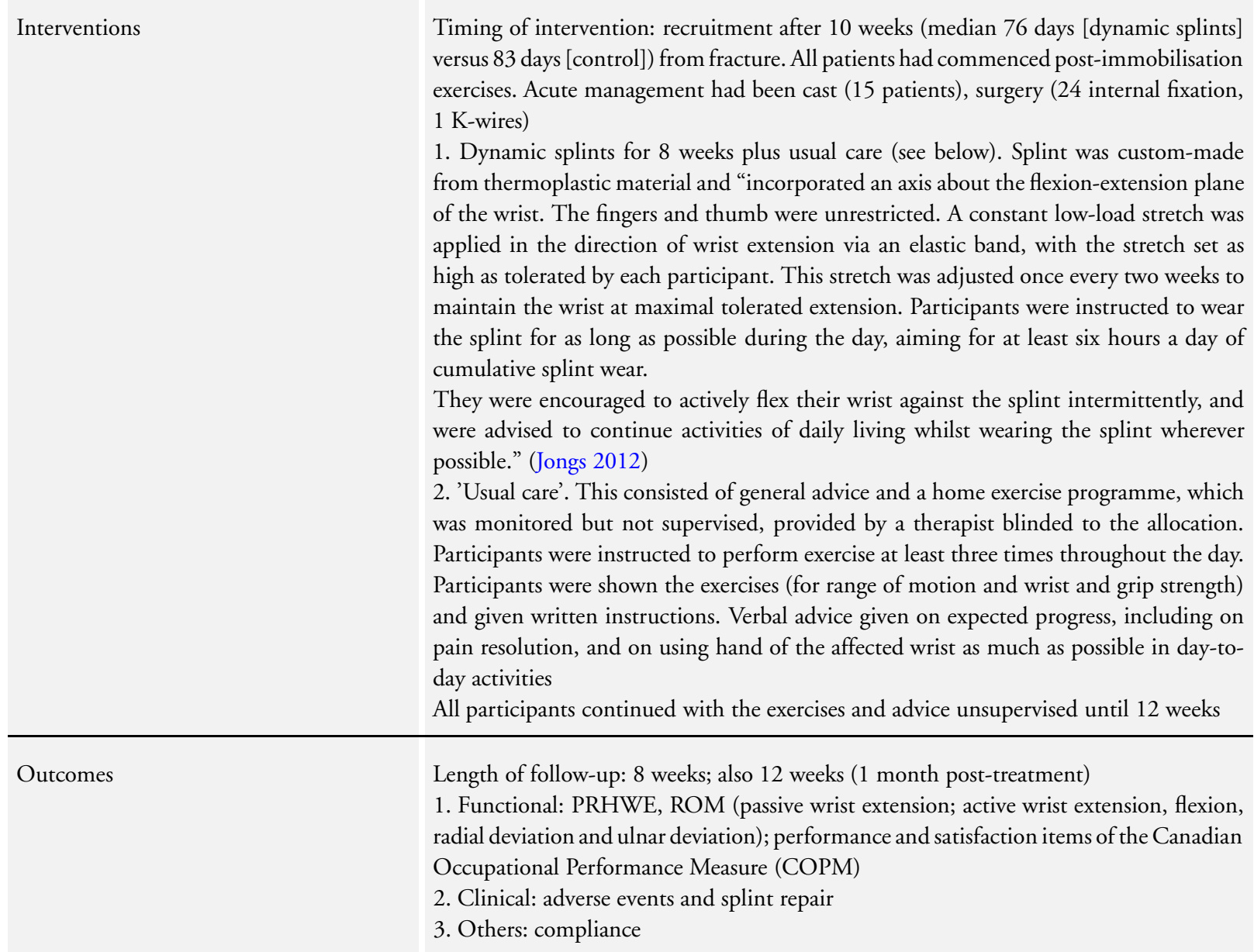

Funding, ethics and patient consent

No mention of funding but no commercial company with direct involvement; sponsor was the University of Sydney. Ethical approval reported

Informed consent was obtained from all participants

Notes

One participant who was randomly allocated to dynamic splint group ended up in the control group within 10 minutes of allocation because of an unexplained error Perprotocol and intention-to-treat analyses were reported

\section{Risk of bias}

Bias

Random sequence generation (selection Low risk bias)

\section{Support for judgement}

"a computerised blocked randomisation sequence was generated prior to the commencement of the trial by an independent offsite person" 
Jongs 2012 (Continued)

\begin{tabular}{|c|c|c|}
\hline Allocation concealment (selection bias) & Low risk & $\begin{array}{l}\text { "Participants' allocations were placed in } \\
\text { opaque sealed and sequentially numbered } \\
\text { envelopes that were held off-site. A partic- } \\
\text { ipant was considered to have entered the } \\
\text { trial once his/her envelope was opened" }\end{array}$ \\
\hline $\begin{array}{l}\text { Blinding of participants and personnel } \\
\text { (performance bias) } \\
\text { Subjective outcomes }\end{array}$ & High risk & $\begin{array}{l}\text { Participants and physiotherapists not blind } \\
\text { to use or not of dynamic splints }\end{array}$ \\
\hline $\begin{array}{l}\text { Blinding of participants and personnel } \\
\text { (performance bias) } \\
\text { Objective outcomes }\end{array}$ & High risk & $\begin{array}{l}\text { Participants and physiotherapists not blind } \\
\text { to use or not of dynamic splints }\end{array}$ \\
\hline $\begin{array}{l}\text { Blinding of outcome assessment (detection } \\
\text { bias) } \\
\text { Subjective outcomes }\end{array}$ & High risk & Participants not blinded for PRWE score \\
\hline $\begin{array}{l}\text { Blinding of outcome assessment (detection } \\
\text { bias) } \\
\text { Objective outcomes (functional impair- } \\
\text { ment) }\end{array}$ & High risk & Assessor at 12 weeks was not blinded \\
\hline
\end{tabular}

Blinding of outcome assessment (detection Unclear risk bias)

Complications, number of sessions, return

Unblinded therapist contacted patients to monitor and record adherence: patients to former activity

Incomplete outcome data (attrition bias) Unclear risk Short term follow-up (up to 3 months)

Data provided to check for differences between per-protocol and intention-to-treat analyses (no problem detected); $20 \%$ loss to follow-up but not badly imbalanced between the two groups $(5 / 20$ versus $3 / 20)$

Incomplete outcome data (attrition bias) Unclear risk $\quad$ No long-term follow-up

Longer term follow-up

Selective reporting (reporting bias) Low risk

Major baseline imbalance bias? Unclear risk

Trial reported according to prior trial registration details

\begin{tabular}{l|l|l|}
\hline & \\
\hline $\begin{array}{l}\text { Other performance bias (e.g. differential Low risk } \\
\text { expertise bias) }\end{array}$
\end{tabular}

There were baseline differences in participant characteristics (e.g. gender: $4 / 19$ versus $8 / 21$ males; surgery: $9 / 19$ versus $16 /$ 21) but similar results for baseline measurements of outcome measures

expertise bias)

Comparable

Rehabilitation for distal radial fractures in adults (Review)

Copyright $\Subset 2015$ The Cochrane Collaboration. Published by John Wiley \& Sons, Ltd. 
Assessor blinding: yes for objective measures

Intention-to-treat analysis: baseline data not given for one non-compliant participant Loss to follow-up: 1

Participants
Royal Adelaide Hospital, Adelaide, Australia

40 participants

Inclusion criteria: distal radial fracture treated with plaster cast or pins and plaster cast, informed consent

Exclusion criteria: inability to understand written / spoken English, previous wrist fracture on affected side within last 20 years or any time if residual impairment, concurrent ipsilateral upper limb fracture, open reduction and internal fixation

Classification: $\mathrm{AO}$

Sex: 27 female $(68 \%)$

Age: mean 53 years

Assigned: 20/20 [passive mobilisation / control]

Assessed: 19/20 (at 6 weeks)
Timing of intervention: following pins and plaster ( 5 versus 8$)$ or plaster cast (14 versus 12) removal (approximately 6 weeks immobilisation).

All participants attended physiotherapy for initial treatment - standardised advice on fracture protection, swelling control, skin care and functional activities. Instructed and asked to practice a home exercise programme - active exercises, soft tissue stretches, stabilising exercises, gentle grip strengthening. All provided with a booklet outlining advice and illustrating exercises. All 3 physiotherapists were experienced in hand therapy 1. 6-week course of passive mobilisation; grading left to physiotherapists. Twice weekly for first 3 weeks and once weekly for next 3 weeks

2. Review at 1 week. Subsequent appointments at physiotherapist's discretion for monitoring and any correction. Always, progression and assessment at 3 weeks and assessment at 6 weeks

Outcomes

Length of follow-up: 6 weeks; also 3 weeks

1. Functional: subjective pain and functional disability, ability to perform 6 functional tests, grip strength, ROM (pronation; supination; flexion; extension; radial deviation; ulnar deviation), thumb motion, web space

2. Clinical: complications (continuing, newly occurring): carpal tunnel syndrome, malunion, marked stiffness and dysfunction of wrists and fingers (CRPS-1?)

3. Resources: Number of attendances of physiotherapy, costs

Funding, ethics and patient consent

Approval from hospital ethics committee noted. No mention of funding

Written informed consent

Notes

Further details of trial received from Sandra Kay 13 \& 17 August 2001

\section{Risk of bias}




\begin{tabular}{l|l|l}
$\begin{array}{l}\text { Random sequence generation (selection } \\
\text { bias) }\end{array}$ & Low risk & $\begin{array}{l}\text { " } 40 \text { patients were included in the study and } \\
\text { randomly allocated to one of two groups } \\
\text { by means of a computer generated random } \\
\text { numbers table" }\end{array}$ \\
\hline Allocation concealment (selection bias) & Low risk & $\begin{array}{l}\text { "40 patients were included in the study and } \\
\text { randomly allocated to one of two groups } \\
\text { by means of a computer generated random } \\
\text { numbers table" } \\
\text { Email from trialist: "Yes I consider ran- } \\
\text { domisation was concealed at the time of } \\
\text { randomisation" }\end{array}$
\end{tabular}

Blinding of participants and personnel High risk

Participants and personnel not blinded

(performance bias)

Subjective outcomes

Blinding of participants and personnel High risk

(performance bias)

Participants and personnel not blinded

Objective outcomes

Blinding of outcome assessment (detection Unclear risk

bias)

Unknown effect but probably not an issue

Subjective outcomes

that the participants weren't blinded

Blinding of outcome assessment (detection
bias)
Objective outcomes (functional impair-
ment)

ment)

Blinding of outcome assessment (detection Low risk

bias)

Complications, number of sessions, return

to former activity

\begin{tabular}{l|l|l}
\hline $\begin{array}{l}\text { Incomplete outcome data (attrition bias) } \\
\text { Short term follow-up (up to } 3 \text { months) }\end{array}$ & Low risk & Only one withdrawal \\
\hline $\begin{array}{l}\text { Incomplete outcome data (attrition bias) } \\
\text { Longer term follow-up }\end{array}$ & Unclear risk & No longer-term follow-up \\
\hline Selective reporting (reporting bias) & Low risk & $\begin{array}{l}\text { No protocol available but there had been } \\
\text { one for the trial and selective reporting } \\
\text { seems very unlikely }\end{array}$ \\
\hline
\end{tabular}

"Patients were assessed prior to commencing physiotherapy, and three and six weeks later, by an independent experienced hand physiotherapist who was unaware of the patient's allocated group"

Outcomes unlikely to be affected by any deficiencies in blinding
Major baseline imbalance bias?
Low risk
Low risk
No problems detected

Rehabilitation for distal radial fractures in adults (Review) 
Other performance bias (e.g. differential Low risk

No problems detected expertise bias)

Kay 2008

Methods

Method of randomisation: use of computer generated random numbers table, administered remotely by an independent person

Assessor blinding: yes for objective measures

Intention-to-treat analysis: no problems detected

Loss to follow-up: 9 (at 6 weeks)

Participants

Royal Adelaide Hospital, Australia

56 participants

Inclusion criteria: distal radial fracture treated with plaster cast or pins and plaster cast, informed consent

Exclusion criteria: unwilling or unable to participate; unable to understand written or spoken English; bilateral wrist fractures or a concurrent injury to the affected limb; previous wrist fracture on the affected side with residual loss of range of motion or function; had a concurrent ipsilateral limb injury, pre-existing inflammatory joint condition, or were managed with internal or external fixation

Classification: AO (breakdown by extra-articular; partial and complete articular fractures)

Sex: 39 female $(70 \%)$

Age: mean 55.4 years

Assigned: 28/28 [physiotherapy/control]

Assessed: 27/20 (at 6 weeks)

Interventions

Timing of intervention: following pins and cast ( 8 versus 7 ) or cast (20 versus 21 ) removal (mean 6 weeks immobilisation)

Prior care or advice during immobilisation not stated. Subsequent information received from the trialist referred to general advice about hand elevation and active range of motion exercises for fingers, elbow and shoulder if seen by a physiotherapist in the ward setting and probably a general hand-out on plaster cast care provided by a nurse if the cast was applied in the emergency department

1. Physiotherapy comprised a single session with an experienced hand therapist who provided standardised advice on fracture protection, swelling control, skin care and everyday activities and an exercise programme. The exercise programme was progressive, consisting of active range of motion exercises for shoulder, elbow, wrist and hand, soft tissue stretches, isometric stabilising wrist exercises. Gentle forearm/wrist/hand strengthening exercises, including grip exercise, were added from week 3. Participants were asked to demonstrate the exercises to the physiotherapist and were instructed to continue the programme at home. A booklet outlining advice and illustrating the exercises was provided. Participants were also fitted with an elastic threaded cotton sleeve for wrist and forearm for swelling control and comfort and instructed on its application and precautions. Participants were provided with physiotherapist's contact details if needing clarification, reassurance or further advice. Where a complication was noted, the physiotherapist went through the advice in greater detail (personal communication)

2. Control group had no physiotherapy input

Both groups had initial assessment and review (at 3 and 6 weeks) by an experienced hand

Rehabilitation for distal radial fractures in adults (Review) 
therapist who would have detected (picked up on) complications

\begin{tabular}{|c|c|}
\hline Outcomes & $\begin{array}{l}\text { Length of follow-up: } 6 \text { weeks; also } 3 \text { weeks } \\
\text { 1. Functional: PRWE (pain and function), QuickDASH general, work, sports), grip } \\
\text { strength, ROM (pronation; supination; flexion; extension; radial deviation; ulnar devi- } \\
\text { ation), thumb motion, web space } \\
\text { 2. Clinical: complications but } 24 / 27 \text { apparent at time of initial measurement (finger } \\
\text { stiffness / swelling in 15; shoulder / neck pain and stiffness in 9); thus an additional } 3 \\
\text { were detected } \\
\text { 3. Others: participant satisfaction with intervention, compliance (just physiotherapy } \\
\text { group), participants requesting physiotherapy after } 6 \text { weeks }\end{array}$ \\
\hline Funding, ethics and patient consent & $\begin{array}{l}\text { Approval from hospital ethics committee noted. Support from RAH Allied Health Re- } \\
\text { search Grant and Australian Hand Therapy Association Research Scholarship } \\
\text { Informed consent }\end{array}$ \\
\hline Notes & $\begin{array}{l}\text { This was previously listed as Kay } 2003 \text { (ongoing study). Initial plans included a } 3 \text { month } \\
\text { follow-up, separate measure of pain (VAS). The trial was held back in order to resolve } \\
\text { some issues relating to inter-rater reliability testing for ROM, staff shortages and training } \\
\text { Received information from Sandra Kay (27 May 2015) on prior treatment, treatment for } \\
\text { detected complications, and details of the pre- and post-randomisation complications }\end{array}$ \\
\hline
\end{tabular}

Risk of bias

\begin{tabular}{|c|c|c|}
\hline Bias & Authors' judgement & Support for judgement \\
\hline $\begin{array}{l}\text { Random sequence generation (selection } \\
\text { bias) }\end{array}$ & Low risk & $\begin{array}{l}\text { "A computer-generated randomisation ta- } \\
\text { ble was kept by an independent person who } \\
\text { was remote from the area where assessment } \\
\text { occurred, and group allocation was revealed } \\
\text { by a phone call" }\end{array}$ \\
\hline
\end{tabular}

Allocation concealment (selection bias) Low risk

Blinding of participants and personnel High risk (performance bias)

Subjective outcomes

Blinding of participants and personnel High risk (performance bias)

Objective outcomes "allocation by contacting the primary researcher who is at a central administration site and has an allocation schedule which is referred to once patient's eligibility and willingness to participate is ascertained" (from study registration)

Participants nor hand therapist were not blinded

Participants nor hand therapist were not blinded 


\begin{tabular}{l|l|l}
\hline $\begin{array}{l}\text { Blinding of outcome assessment (detection } \\
\text { bias) }\end{array}$ & High risk & Participants were not blinded \\
Subjective outcomes & \\
\hline
\end{tabular}

Blinding of outcome assessment (detection Low risk bias)

Objective outcomes (functional impairment)
"Therapist-rated outcome measures were collected by an experienced hand physiotherapist (MMcM) who was blinded to group allocation. To maintain blinding, participants were asked not to discuss any aspect of the trial with the assessor"

Outcomes not used for reporting

Blinding of outcome assessment (detection Unclear risk bias)

Complications, number of sessions, return

to former activity

Incomplete outcome data (attrition bias) High risk

Short term follow-up (up to 3 months)
Greater losses in the control group (1/ 28 [physiotherapy] versus $8 / 28$ [control]) could be a source of bias

Incomplete outcome data (attrition bias) Unclear risk $\quad$ No longer-term outcomes

Longer term follow-up

Selective reporting (reporting bias) Unclear risk

The separate categories of PRWE and QuickDASH not mentioned in Methods or trial registration

$\begin{array}{lll}\text { Major baseline imbalance bias? } & \text { Low risk } & \text { None }\end{array}$

Other performance bias (e.g. differential Unclear risk expertise bias)

Insufficient information on advice interventions before cast removal and inclusion in trial and subsequently

\section{Knygsand-Roenhoej 2011}

\begin{tabular}{ll} 
Methods & $\begin{array}{l}\text { Method of randomisation: no details - mention of consecutive recruitment } \\
\text { Assessor blinding: yes for objective measures }\end{array}$ \\
& $\begin{array}{l}\text { Intention-to-treat analysis: baseline data not given for one non-compliant participant } \\
\text { Loss to follow-up: } 1 \text { (excluded) }\end{array}$ \\
\hline Participants & $\begin{array}{l}\text { Department of Occupational Therapy and Physiotherapy, Aarhus University Hospital, } \\
\text { Aarhus, Denmark } \\
\text { 30 participants }\end{array}$ \\
$\begin{array}{l}\text { Inclusion criteria: aged } 18 \text { years or older, unilateral post-distal radius fracture treated } \\
\text { with a plaster cast or internal or external fixation, subacute oedema } 4 \text { to } 10 \text { weeks post- } \\
\text { trauma/surgery and a } 60 \mathrm{~mL} \text { or more in volume difference between the upper extremities. } \\
\text { Informed consent }\end{array}$
\end{tabular}


Exclusion criteria: mental impairments preventing participation, infection, disease of the internal organs, and/or presence of lymphedema

Classification: Colles \& Smith's

Data for 29 participants (11 treated conservatively, 9 internal fixation, 9 external fixation) Sex: 21 female $(72 \%)$

Age: mean 48 years

Assigned: 15/15 [MEM/traditional oedema treatments]

Assessed: 14/15 (at 6 months); 13/15 (at 9 weeks, COPD measure)

Timing of intervention: 4 to 10 weeks post-conservative ( 4 versus 7 ) or surgical (10 versus 8) treatment (average 47 days post-injury)

1. Modified 'manual edema mobilization' (MEM) consists of a specific massage technique aimed at promoting the flow of lymph, exercises, exercises during the massage sequence, deep diaphragm breathing, a low-stretch bandage system if needed, and a one-handed MEM home programme. At the first session, MEM was only performed to the trunk region and sometimes included pump points to the elbow region. Subsequent sessions involved MEM to the trunk and pump points distally to the wrist and MEM to the hand region depending on the patient's clinical picture.Session took approximately 30 minutes. Patients were instructed to wear an isotoner open-finger compression glove except when receiving massage or undertaking personal hygiene. If the oedema became hard or brawny, the patient was instructed to use a multilayered low-stretch bandage and/or a chip bag together with the isotoner glove. At home, patients were instructed to perform a MEM exercise programme and a program for functional training. All treated by principal investigator

2. 'Traditional oedema treatment': elevation, compression, and functional training. Patient's digits and hand were wrapped in a compression bandage. Patients engaged in activity where arm was above shoulder height for at least 10 minutes; if possible, further treatment was performed above heart height. A Flowtron intermittent compression system with three chambers was used for 20 minutes to decrease hand/arm oedema. At home, patients were instructed to perform their home programme for oedema and mobility. During the night, all patients used an isotoner open-finger compression glove. All treated by the same experienced occupational therapist

For both groups, oedema treatment was performed three times a week for four weeks and then twice a week for two weeks. After the initial 6 weeks, treatment was continued until function reached a level that reflected the patients' needs and the therapist's assessment of functional requirements. All patients had ROM and strengthening therapy according "to need" at the hospital and as home exercises. All patients received identical instructions for home exercises to improve hand and wrist mobility, including one exercise for oedema

1. Functional: ADL assessed using a investigator-designed questionnaire for bilateral activities

(QBA) - in a structured interview, participants were asked about four activities: tying their shoelaces, eating with a knife and fork, peeling potatoes, and cutting a slice of bread ( 0 to $16 ; 0=$ cannot perform specific activity), pain (over 3 days: VAS), performance and satisfaction items of the Canadian Occupational Performance Measure (COPM) (at 9 weeks), active ROM ulnar digit (not used in this review)

2. Clinical: oedema, complications, treatment of oedema

3. Others: number of occupational therapy sessions; poor compliance (as reason for post- 
Knygsand-Roenhoej 2011 (Continued)

randomisation exclusion)

Funding, ethics and patient consent

No mention of funding. Regional ethics committee approval reported. Also reference to the Declaration of Helsinki

Oral and written informed consent was obtained from all participants

Notes

Sent request for information on method of randomisation and missing participant on 10 May 2013

Risk of bias

\begin{tabular}{l|l|l}
\hline Bias & Authors' judgement & Support for judgement \\
\hline $\begin{array}{l}\text { Random sequence generation (selection } \\
\text { bias) }\end{array}$ & Unclear risk & $\begin{array}{l}\text { "Patients were consecutively included } \\
\text { when a subacute edema was diagnosed and } \\
\text { randomly assigned into two groups". No } \\
\text { information }\end{array}$ \\
\hline Allocation concealment (selection bias) & Unclear risk & $\begin{array}{l}\text { "Patients were consecutively included } \\
\text { when a subacute edema was diagnosed and } \\
\text { randomly assigned into two groups". No } \\
\text { information }\end{array}$ \\
\hline
\end{tabular}

Blinding of participants and personnel High risk (performance bias)

No blinding of the two treatment providers Subjective outcomes or trial participants

Blinding of participants and personnel High risk (performance bias)

No blinding of the two treatment providers Objective outcomes or trial participants

Blinding of outcome assessment (detection Unclear risk bias)

Subjective outcomes

Trial participants were not blinded. It is unclear whether this would have affected their ratings

Blinding of outcome assessment (detection Low risk bias)

Objective outcomes (functional impairment)

The two "experienced occupational therapists" evaluating these outcomes "were blinded to the treatment groups because patients were instructed not to tell which treatment they received and to remove the isotoner glove and/or the low-stretch bandage at home before their visit with the evaluation therapist"

Blinding of outcome assessment (detection Unclear risk bias)

No indication of blinding for assessing

Complications, number of sessions, return

to former activity

Rehabilitation for distal radial fractures in adults (Review) 


\section{Knygsand-Roenhoej 2011 (Continued)}

\begin{tabular}{|c|c|c|}
\hline $\begin{array}{l}\text { Incomplete outcome data (attrition bias) } \\
\text { Short term follow-up (up to } 3 \text { months) }\end{array}$ & Unclear risk & $\begin{array}{l}\text { One excluded from 'MEM' group for } \\
\text { non-compliance and one missing (not ex- } \\
\text { plained) from same group for Canadian } \\
\text { Occupational Performance Measure results }\end{array}$ \\
\hline
\end{tabular}

Incomplete outcome data (attrition bias) Low risk

Longer term follow-up

Selective reporting (reporting bias)

Unclear risk
One excluded from 'MEM' group for noncompliance

No protocol and inadequate reporting of some subjective outcomes

No problems detected

Major baseline imbalance bias? Low risk

"To reduce the inherent bias in the de-

Other performance bias (e.g. differential Unclear risk expertise bias) sign due to different quality of treatment programs, we chose experienced occupational therapists for both groups." However, functional treatment provided by therapist started at least one week later in the 'MEM' group

Krischak 2009

Methods
Method of randomisation: blocked randomisation stratified by age

Assessor blinding: no

Intention-to-treat analysis: no, 2 post-randomisation exclusions (additional physiotherapy sessions)

Loss to follow-up: 2 exclusions (at 6 weeks)

Participants

Clinic for Traumatology, Hand, Plastic, and Reconstructive Surgery, University of Ulm, Ulm, Germany

48 participants

Inclusion criteria: patients treated with open reduction and internal fixation with a volar locking plate for a distal radius fracture; informed consent

Exclusion criteria: < 18 years, uncooperative, lacked cognitive capacity for trial participation, dependent in the requirements of daily life (e.g. home care needed), psychiatric illness, bone disease underlying fracture (i.e. bone metastasis, osteolysis), previous fracture near the wrist, carpal tunnel syndrome on the injured side, inflammatory joint disease (e.g. rheumatoid arthritis, gout), or "reflex sympathetic dystrophy"

Classification: AO (A, B and C; and first level of subcategories)

Data for 46 participants

Sex: 30 female $(65 \%)$

Age: mean 55 years (range 18 to 76 )

Assigned: 24/24 [physiotherapy / home exercise]

Assessed: 23/23 (at 6 weeks) 
Krischak 2009 (Continued)

\begin{tabular}{|c|c|}
\hline Interventions & $\begin{array}{l}\text { Timing of intervention: one week post-surgery. All participants put in wrist splint after } \\
\text { surgery for } 2 \text { weeks. The splint was removed for the therapy } \\
\text { 1. Physiotherapy. Prescription for } 12 \text { sessions lasting } 20 \text { to } 30 \text { minutes each, over a 6- } \\
\text { week period. Participants free to choose their own therapist; and therapist to choose type } \\
\text { of therapy based on their assessment of the patient. "As usual", therapists were instructed } \\
\text { to implement exercises that could be done by the patients unassisted at home } \\
\text { 2. Instruction for home exercises. Detailed instructions and demonstrations on the home } \\
\text { exercise programme provided in person and an exercise guidance booklet provided. } \\
\text { The contents instructed the patients about the type of exercises, repetitions, intensity, } \\
\text { training, and rest phases, as well as including a diary-type weekly plan (illustrated in } \\
\text { report). Three to five exercises were grouped in units requiring approximately } 20 \text { minutes. } \\
\text { Instructions for } 2 \text { training units, to be performed once in the morning and once in } \\
\text { the evening each day. Patients kept training dairy. Exercises were grouped by week: } \\
\text { for first } 2 \text { weeks, priority was given to pain reduction and reduction of postoperative } \\
\text { oedema; after the second week, passive stretching exercises were introduced, early active } \\
\text { movements were added, without resistance, including stretching and spreading fingers, } \\
\text { making a fist, forearm stretching, and bending and stretching the elbow, as well as } \\
\text { abduction/adduction and external/internal rotation of the arm. Starting after the second } \\
\text { week, exercises from the PNF (proprioceptive neuromuscular facilitation) technique } \\
\text { were introduced. In the fifth week, dynamic muscle exercises against light resistance were } \\
\text { increased. (Thus approximately } 280 \text { minutes exercise aimed for each week) }\end{array}$ \\
\hline Outcomes & $\begin{array}{l}\text { Length of follow-up: } 6 \text { weeks } \\
\text { 1. Functional: PRWE, grip strength ( } \% \text { of uninjured side - adjusted for hand dominance) } \\
\text {, ROM (\% of uninjured side) (extension-flexion, ulnar-radial abduction (deviation), } \\
\text { supination-pronation) } \\
\text { 2. Others: compliance (sessions attended; home exercises done); costs of physiotherapy } \\
\text { sessions }\end{array}$ \\
\hline
\end{tabular}

Funding, ethics and patient consent

No mention of funding but no commercial company with direct involvement. Local ethics committee approval reported

Written informed consent was obtained from all participants

Notes

Sent request for information on method of randomisation and details of the additional physiotherapy received by the two excluded participants on 4 May 2013; email returned, sent request in post on 7 May 2013

\section{Risk of bias}

Bias

Random sequence generation (selection Unclear risk bias)
Authors' judgement
Unclear risk

\section{Support for judgement}

"A randomized selection process based on age was used to sort each patient into 1 of 2 postoperative programs. The randomized selection process based on age was done through block randomization"

No details available 
Krischak 2009 (Continued)

\begin{tabular}{|c|c|c|}
\hline $\begin{array}{l}\text { Blinding of participants and personnel } \\
\text { (performance bias) } \\
\text { Subjective outcomes }\end{array}$ & High risk & No blinding \\
\hline $\begin{array}{l}\text { Blinding of participants and personnel } \\
\text { (performance bias) } \\
\text { Objective outcomes }\end{array}$ & High risk & No blinding \\
\hline $\begin{array}{l}\text { Blinding of outcome assessment (detection } \\
\text { bias) } \\
\text { Subjective outcomes }\end{array}$ & High risk & No blinding \\
\hline $\begin{array}{l}\text { Blinding of outcome assessment (detection } \\
\text { bias) } \\
\text { Objective outcomes (functional impair- } \\
\text { ment) }\end{array}$ & High risk & No blinding nor independent assessment \\
\hline $\begin{array}{l}\text { Blinding of outcome assessment (detection } \\
\text { bias) } \\
\text { Complications, number of sessions, return } \\
\text { to former activity }\end{array}$ & Low risk & $\begin{array}{l}\text { Outcomes unlikely to be affected by lack } \\
\text { of blinding }\end{array}$ \\
\hline $\begin{array}{l}\text { Incomplete outcome data (attrition bias) } \\
\text { Short term follow-up (up to } 3 \text { months) }\end{array}$ & Unclear risk & $\begin{array}{l}\text { Exclusion of one from each group unlikely } \\
\text { to result in bias. However, grip strength and } \\
\text { ROM outcomes inadequately reported }\end{array}$ \\
\hline $\begin{array}{l}\text { Incomplete outcome data (attrition bias) } \\
\text { Longer term follow-up }\end{array}$ & Unclear risk & No longer-term follow-up \\
\hline Selective reporting (reporting bias) & Unclear risk & No trial registration or protocol available \\
\hline Major baseline imbalance bias? & Low risk & None \\
\hline $\begin{array}{l}\text { Other performance bias (e.g. differential } \\
\text { expertise bias) }\end{array}$ & Unclear risk & $\begin{array}{l}\text { Unknown. Pragmatic trial but no descrip- } \\
\text { tion of physiotherapy provided or if home } \\
\text { exercises were encouraged in the physio- } \\
\text { therapy group }\end{array}$ \\
\hline
\end{tabular}



fracture (Frykman classification)

Assessor blinding: only orthopaedic surgeon assessing radiographic parameters

Intention-to-treat analysis: yes

Loss to follow-up: 0 (at 12 weeks)

Participants
Department of Occupational Therapy, National Cheng Kung University Hospital, Tainan, Taiwan

22 participants

Inclusion criteria: patients treated for a distal radius fracture with an Hoffman external fixator. Informed consent, aged over 50 years old, no previous history of hand or forearm injury to either upper limb; absence of rheumatism or osteoarthritis of the hands; no other neurological deficits or severe soft tissue damage

Exclusion criteria: as inferred from the above

Classification: Frykman

Data for 22 participants

Sex: 15 female $(68 \%)$

Age: mean 62.1 years (range not given)

Assigned: 11/11 [early digit mobilisation / control]

Assessed: 11/11 (at 12 weeks) (see Notes)

\section{Interventions}

Outcomes
Timing of intervention: post-surgery

1. Early digit mobilisation: Three $\mathrm{x}$ 45-minute session per week until removal of external fixator. Week 1 and 2: Massage, passive stretching and passive and active ROM of uninvolved joints; wound care and infection control. Week 3 and 4: Massage, passive stretching and passive and active ROM of uninvolved joints; ADL training; pain free isometric and concentric exercise for the digits. Week 5 and 6: as previous, plus tendon gliding exercise of the digits. Treatment was by single named occupational therapist 2. Control: Usual rehabilitation programme. Week 1 and 2: Elevation of the involved limb; active ROM of shoulder and elbow joints; Week 3 and 4: Elevation of the involved limb, pain-free active ROM, active ROM of shoulder, elbow and hand joints

After the external fixator was removed (six weeks after fracture), the patients in both groups received a standard progressive rehabilitation programme, including scar management, active and passive wrist ROM, wrist joint mobilisation and strengthening, until 12 weeks after surgical fixation
Length of follow-up: 12 weeks; also at 1, 3 and 7 weeks ( 1 week post-removal of external fixation)

1. Functional: Manual Ability Measure-36 (MAM-36)(Chen 2010), grip strength (\% of uninjured side), pinch strength (palmar pinch and 'three-jaw chuck' pinch), manual dexterity (Purdue Pegboard test), thumb and finger joint ROM ('maximal workspace' and 'dynamic goniometry')

2. Others: radiological outcome (radial tilt, radial height shortening, volar tilt)
Partially funded by National Science Council grant, Taiwan

Local ethics committee approval reported

Written informed consent was obtained from all participants. 
Kuo 2013

Notes

Response from lead author (April 12015 ) in response to query sent on MAM-36 scores (> 144 top limit) and follow-up schedule

1. "Yes, the original MAM-36 is a 36-items questionnaire. For some cultural reasons, we used a modified Taiwanese version MAM which is a 45 items (please see the attachment) . Because we did not find the literature with regard to this version in English, we cited her report regarding MAM-36 in 2010"

2. He clarified that there was no loss to follow-up and the statement in the Discussion "it should be noted that only $73 \%$ of the participants originally recruited completed the scheduled intervention or the follow-up examinations within the valid duration approved by the institutional review board" referred to the loss of eight from recruitment because they "expressed that they could not completely follow our schedule with regard to the intervention"

Risk of bias

\begin{tabular}{l|l|l}
\hline Bias & Authors' judgement & Support for judgement \\
\hline $\begin{array}{l}\text { Random sequence generation (selection } \\
\text { bias) }\end{array}$ & Unclear risk & $\begin{array}{l}\text { "The participants were randomly assigned } \\
\text { to one of two groups - the early digit mobi- } \\
\text { lization group or the control group - by us- } \\
\text { ing a computerized random number sheet } \\
\text { with the group assignment written in the } \\
\text { sealed envelopes. Randomization was ac- } \\
\text { complished by stratifying according to the } \\
\text { age, gender, injured hand (right or left) and } \\
\text { severity of fracture (Frykman classification) } \\
\text { of the participants." } \\
\text { The stratification is unlikely to have } \\
\text { worked with the small numbers involved }\end{array}$ \\
\hline Allocation concealment (selection bias) & Unclear risk & $\begin{array}{l}\text { Use of sealed envelopes. Insufficient infor- } \\
\text { mation on safeguards }\end{array}$
\end{tabular}

Blinding of participants and personnel High risk (performance bias)

Neither participants nor personnel provid-

Subjective outcomes

ing care were blinded

Blinding of participants and personnel High risk (performance bias)

Neither participants nor personnel provid-

Objective outcomes

ing care were blinded

Blinding of outcome assessment (detection High risk

Trial participants were not blinded

bias)

Subjective outcomes

Blinding of outcome assessment (detection Unclear risk

bias)

Objective outcomes (functional impair-

ment)

Rehabilitation for distal radial fractures in adults (Review)

Copyright $\odot 2015$ The Cochrane Collaboration. Published by John Wiley \& Sons, Ltd.

Not blinded but the systematic approach is likely to protect against detection bias 


\author{
Blinding of outcome assessment (detection Low risk \\ bias) \\ Complications, number of sessions, return \\ to former activity
}

The radiographic parameters, including those to assess malunion, "were measured by an orthopaedic surgeon (CLL) who was blind to the group that each participant was in"

No loss to follow-up
Incomplete outcome data (attrition bias)
Short term follow-up (up to 3 months)

Low risk

Unclear risk

Incomplete outcome data (attrition bias)

Longer term follow-up

Selective reporting (reporting bias)

Low risk

Major baseline imbalance bias?

Unclear risk
No longer-term follow-up

No protocol but clearly a systematic approach to outcome assessment

None of the tests for differences between the groups were significant. There were, however, more women in the control group, the participants of which were on average 5 years older

All had same method of surgery performed by the same surgeon, with rehabilitation by the same occupational therapist and a common programme after fixator removal

\section{Lazovic 2012}

Methods

Participants
Method of randomisation: alternation

Assessor blinding: only orthopaedic surgeon assessing radiographic parameters

Intention-to-treat analysis: yes

Loss to follow-up: 0 (at 2 to 3 days post-end of cast immobilisation)

Physical Medicine and Rehabilitation Clinic, Clinical Centre, Niš, Serbia

60 participants

Inclusion criteria: over the age of 55 years, extra-articular displaced stable distal radius fracture treated with reduction and cast immobilisation, low impact fracture, intact cognitive function, no limitation of wrist and hand function before injury, informed consent

Exclusion criteria: bilateral wrist fractures, previous fracture of the affected or unaffected wrist, additional wrist fracture, inflammatory osteoarthritis, peripheral vascular disease, and contra-indications to PEMF (patients with auxiliary organs, i.e. pace makers, all kinds of mycoses and tumours, intestinal haemorrhage, epilepsy, hyperthyroidism and acute infection).

Classification: none

Sex: all female (100\%)

Age: mean 66.2 years (range 55 to 78 ) 
Lazovic 2012 (Continued)

Assigned: 30/30 [PEMF / control]

Assessed: $30 / 30$ (at 2 to 3 days post-end of cast immobilisation)

Interventions

Timing of intervention: mean 8.7 days from fracture; after being directed for rehabilitation after clinical and radiographic control at 7 days after fracture

1. Pulsed electromagnetic field (PEMF): at $25 \mathrm{~Hz}$ with a field intensity of $6 \mathrm{mT}$. PEMF provided 5 days a week for 2 weeks, 30 minutes daily

2. Control: no PEMF (other therapy)

Each participant was taught and given instructions for a home exercise programme (active shoulder, elbow and finger mobilisation exercises, active fisting and thumb opposition, as allowed by cast) performed twice a day for 20 minutes during cast immobilisation. After cast removal (mean 28 days, range 25 to 35 days) they were instructed to perform light activities in the pain-free range of movement until the follow-up ( 2 to 3 days). They were also advised to elevate the wrist if they noted increasing oedema

Outcomes

Length of follow-up: 2 to 3 days after cast removal

1. Functional: PRWE function score; PRWE pain score; ROM (pronation; supination; flexion; extension; radial deviation; ulnar deviation)

2. Clinical: PRWE pain score; complications

2. Others: oedema

Funding, ethics and patient consent

No statement on funding

Local ethics committee approval reported

Written informed consent was obtained from all participants

Notes

Risk of bias

Bias

Authors' judgement

Support for judgement

Random sequence generation (selection High risk bias)

"The patients were then allocated to either the PEMF group $(\mathrm{n}=30)$, or control group $(\mathrm{n}=30)$ alternately"

Allocation concealment (selection bias) High risk

Alternation (predicable allocation)

Blinding of participants and personnel High risk

Not blinded: No sham (placebo) control

(performance bias)

Subjective outcomes

Blinding of participants and personnel High risk

Not blinded: No sham (placebo) control (performance bias)

Objective outcomes

Blinding of outcome assessment (detection High risk

Participants were not blinded

bias)

Subjective outcomes

Rehabilitation for distal radial fractures in adults (Review)

Copyright @ 2015 The Cochrane Collaboration. Published by John Wiley \& Sons, Ltd. 
Lazovic 2012 (Continued)

\begin{tabular}{l|l}
$\begin{array}{l}\text { Blinding of outcome assessment (detection } \\
\text { bias) }\end{array}$ & Low risk \\
$\begin{array}{l}\text { Objective outcomes (functional impair- } \\
\text { ment) }\end{array}$ & $\begin{array}{l}\text { All examined parameters were assessed by } \\
\text { one of the authors blinded to group alloca- } \\
\text { tion }\end{array}$ \\
\hline
\end{tabular}

\begin{tabular}{l|l|l}
$\begin{array}{l}\text { Blinding of outcome assessment (detection } \\
\text { bias) }\end{array}$ & Low risk & $\begin{array}{l}\text { All examined parameters were assessed by } \\
\text { one of the authors blinded to group alloca- } \\
\text { Complications, number of sessions, return } \\
\text { to former activity }\end{array}$
\end{tabular}

\begin{tabular}{|c|c|c|}
\hline $\begin{array}{l}\text { Incomplete outcome data (attrition bias) } \\
\text { Short term follow-up (up to } 3 \text { months) }\end{array}$ & Unclear risk & $\begin{array}{l}\text { No loss to follow-up. However, follow-up } \\
\text { was too short }\end{array}$ \\
\hline
\end{tabular}

\begin{tabular}{l|ll}
\hline $\begin{array}{l}\text { Incomplete outcome data (attrition bias) } \\
\text { Longer term follow-up }\end{array}$ & Unclear risk & No long-term follow-up \\
\hline Selective reporting (reporting bias) & Unclear risk & $\begin{array}{l}\text { No protocol although data systematically } \\
\text { collected and reported. No grip strength }\end{array}$ \\
\hline Major baseline imbalance bias? & Unclear risk & $\begin{array}{l}\text { Small age difference but unlikely to be clini- } \\
\text { cally important. No description of fractures }\end{array}$ \\
\hline $\begin{array}{l}\text { Other performance bias (e.g. differential } \\
\text { expertise bias) }\end{array}$ & Low risk & $\begin{array}{l}\text { All therapy comparable except for the in- } \\
\text { tervention }\end{array}$ \\
\hline
\end{tabular}

\section{Maciel 2005}

Methods
Method of randomisation: use of sealed envelopes (concealment stated in report, envelope picked by trial participants after their giving consent)

Assessor blinding: yes, independent and blinded examiner for objective measures Intention-to-treat analysis: problems though reported as done. No baseline measurement and thus data for 4 excluded ("did not enter study": 1 failed to attend, 1 readmitted comorbidity, 1 with CRPS-1 treatment scheduled, 1 failed inclusion) after randomisation Loss to follow-up: 8 (including 1 death, 1 seeking another orthopaedic opinion, and 1 remanipulation under general anaesthesia) (24 weeks) (+4 not 'entered' into trial - see above)

Participants
Western General Hospital, Footscray, Australia

45 participants but baseline data for only 41 participants

Inclusion criteria: distal radial fracture treated with plaster cast (34 participants) or Kwire(s) and plaster cast (7 participants), cast removed, age 18 years or over, ability to understand written and spoken English, willingness to participate

Exclusion criteria: signs or symptoms of "complex regional pain syndrome", documented evidence of psychiatric disorder, pre-existing upper limb inflammatory joint condition, external or internal fixation in situ (apart from K-wire), concurrent upper limb fracture requiring treatment

Classification: AO

Sex: 31 female $(76 \%)$ 
Age: mean 56 years

Assigned: ?/?; baseline data for 23/18 [activity focussed/single session]

Assessed: 19/14 (at 24 weeks)

Interventions

Outcomes

Funding, ethics and patient consent

Notes

Information on this trial was originally presented under Maciel 2002 in the 'Characteristics of ongoing studies' table. Some of the information (especially the outcomes measured) provided by Nick Taylor in 2002 and 2004 was not provided in the full report of this trial

Timing of intervention: following plaster cast removal (3 versus 4 in surgery group; 20 versus 14 in non-surgery group) and plaster cast removal (and on average 44 days immobilisation). Participants were taught routine exercises by a physiotherapist on the day of cast removal. The exercises focussed on the return of active movement to the wrist. All participants received a sheet with information and details of home exercises (Taylor, personal communication)

1. Regular attendance of activity-focussed physiotherapy for up to 6 weeks. The total number of sessions was based on the clinical judgement of the treating physiotherapist in consultant with the patient. Physiotherapy usually stopped on return to regular wrist activity. Activity-focussed physiotherapy involved an assessment and treatment approach that focussed on restoring optimal motor performance of activities that were limited. The emphasis was on skill acquisition. Manual therapy was used to address impairments where these affected the execution of a task. The principles of 'motor learning' were applied as required

2. Single session of advice within one week of entry comprising clarification of exercises from the physiotherapist

Length of follow-up: 24 weeks; also 6 weeks

1. Functional: subjective pain, activity and disability within the PRWE score (higher \% = worse outcome); grip strength, ROM (flexion; extension). (Pronation and supination, ability to make a fist and thumb motion indicated as being recorded in the trial details when ongoing)

2. Clinical: adverse effects

3. Number of attendances of physiotherapy. (Adherence to instructions and home exercises reported in trial details when ongoing)

No mention of funding or ethics

All participants gave informed consent

\section{Risk of bias}

\begin{tabular}{|c|c|c|}
\hline Bias & Authors' judgement & Support for judgement \\
\hline $\begin{array}{l}\text { Random sequence generation (selection } \\
\text { bias) }\end{array}$ & Low risk & $\begin{array}{l}\text { "The participant after gaining consent was } \\
\text { asked to randomly choose a sealed enve- } \\
\text { lope" }\end{array}$ \\
\hline Allocation concealment (selection bias) & Low risk & $\begin{array}{l}\text { "Randomisation was via concealed en- } \\
\text { velopes. The participant after gaining con- } \\
\text { sent was asked to randomly choose a sealed }\end{array}$ \\
\hline
\end{tabular}

Rehabilitation for distal radial fractures in adults (Review) 
Maciel 2005 (Continued)

envelope”

\begin{tabular}{|c|c|c|}
\hline $\begin{array}{l}\text { Blinding of participants and personnel } \\
\text { (performance bias) } \\
\text { Subjective outcomes }\end{array}$ & High risk & Participants and personnel not blinded \\
\hline $\begin{array}{l}\text { Blinding of participants and personnel } \\
\text { (performance bias) } \\
\text { Objective outcomes }\end{array}$ & High risk & Participants and personnel not blinded \\
\hline $\begin{array}{l}\text { Blinding of outcome assessment (detection } \\
\text { bias) } \\
\text { Subjective outcomes }\end{array}$ & High risk & Participants not blinded \\
\hline $\begin{array}{l}\text { Blinding of outcome assessment (detection } \\
\text { bias) } \\
\text { Objective outcomes (functional impair- } \\
\text { ment) }\end{array}$ & Low risk & $\begin{array}{l}\text { "Outcome measures were obtained by an } \\
\text { independent examiner, blind to the group } \\
\text { allocation." Probably safe, although no } \\
\text { mention of safeguards }\end{array}$ \\
\hline
\end{tabular}

Blinding of outcome assessment (detection Low risk bias)

Complications, number of sessions, return

to former activity

Incomplete outcome data (attrition bias) Unclear risk

Short term follow-up (up to 3 months)

Incomplete outcome data (attrition bias) Unclear risk Longer term follow-up

Selective reporting (reporting bias)

Unclear risk

Unclear risk
No data for 4 excluded ("did not enter study") after randomisation. A further 6 lost (4 versus 2) from the 6-week followup. Trial report indicated that the results were not importantly different

No data for 4 excluded ("did not enter study") after randomisation. A further 8 lost (4 versus 4) from the 24-week followup. Trial report indicated that the results were not importantly different

No protocol available but some information (especially the outcomes measured) on this trial before its completion provided by Nick Taylor in 2002 and 2004 was not presented in the full report of this trial

Unlikely but baseline information not available for 4 patients

While the same physiotherapists were involved in both groups, lack of information on other treatment to judge this 

education group (failed to do one or more sessions a week) was excluded from the analyses.

Loss to follow-up: 12 (including 10 withdrawals "due to loss of interest", 1 dropout because of unrelated heath concerns, and 1 post-randomisation exclusion for non-adherence)

Participants
Royal University Hospital in Saskatoon, Saskatchewan, Canada

51 participants (all women)

Inclusion criteria: women, aged 50 years or older, with unilateral distal radial fracture treated conservatively (cast) or surgically (11 participants), under the direction of one orthopaedic surgeon. Informed consent.

Exclusion criteria: any prior upper body injury or joint problem interfering with daily life; any history of upper-extremity neurological problems (e.g. stroke, multiple sclerosis, Parkinson's disease, vestibular disorders, reflex neuropathy); fracture was $>2$ weeks old at the time of the first visit to the clinic; or multiple fractures of the wrist and forearm. Unable to remember any or only 1 or 2 words in the word recall or had an abnormal clock draw test when completing the Mini-Cognitive Assessment Instrument for Dementia Classification: not stated

Sex: 51 female (100\%)

Age: mean 63 years

Assigned: $27 / 24$ [cross-education/control]

Assessed: 19/21 (at 26 weeks). However, one intervention group participant was excluded from the analyses because of inadequate compliance
Timing of intervention: started after randomisation. Participant were treated with cast immobilisation (18 versus 22) or surgical repair then cast immobilisation (9 versus 2 participants). Forearm casting was for a mean of 40 days

1. Standard rehabilitation (see below) plus strength training of opposite hand (crosseducation). Non-fractured hand strength training began immediately post-fracture and was conducted at home 3 times per week for 26 weeks. Strength training was progressive in nature, beginning with 2 sets of 8 repetitions and increasing up to a maximum of 5 sets of 8 repetitions of maximal voluntary effort handgrip contractions as tolerated. Handgrip training was performed using standard handgrip trainers to train finger, hand, and forearm strength. Training was unsupervised and progression in resistance was individually determined; however it was monitored throughout the study by telephone calls and at subsequent visits. Participants recorded adherence in a training log monitored by the researchers

2. Standard rehabilitation (control). Standard rehabilitation protocol included six visits to fracture clinic: at 1, 3, 6, 9, 12 and 26 weeks post-fracture. Adoption of three paperbased exercise protocols (in cast, 6 weeks post-fracture and 9 weeks post-fracture). The orthopedic surgeon coached patients on each of the time-specific protocols at the appropriate time. Standard rehabilitation began with active ROM exercises for the neck, shoulder, elbow, fingers, and thumb while in the cast. After cast removal, exercises focussed on improving active and passive range of motion of the fractured wrist and hand (supination, pronation, flexion, extension). Stretching continued at 9 weeks post-fracture, and strengthening exercises were integrated into the exercise regimen. Strengthen- 
ing exercises were prescribed once per day. Participants were instructed to complete the exercises 10 to 12 times per day. At 12 weeks postfracture, the patients were encouraged to continue with their exercises. All were unsupervised home exercises. Participants were called via telephone bi-weekly and were asked how their wrist was feeling, but there was no monitoring nor participant recording of adherence

In both groups, seeing a physiotherapist was by referral by clinician or on patient initiative

Outcomes

Length of follow-up: 26 weeks; also 9 and 12 weeks

1. Functional: PRWE score (higher \% = worse outcome); grip strength, ROM (flexion and extension; pronation and supination)

2. Clinical: none stated except number having surgery

3. Number of attendances of physiotherapy

Funding, ethics and patient consent

Local ethics approval and operational approval from the health region

All participants gave written informed consent

Notes

Conference abstract presented an interim analysis of 18 women

Risk of bias

\begin{tabular}{|c|c|c|}
\hline Bias & Authors' judgement & Support for judgement \\
\hline $\begin{array}{l}\text { Random sequence generation (selection } \\
\text { bias) }\end{array}$ & Low risk & $\begin{array}{l}\text { "Participants were randomly assigned to } 1 \\
\text { of } 2 \text { groups using a computer random num- } \\
\text { ber generator" }\end{array}$ \\
\hline
\end{tabular}

Allocation concealment (selection bias) Low risk

"Randomization was completed at the first visit to the clinic by a researcher who did not conduct any of the testing procedures"

Blinding of participants and personnel High risk (performance bias)

"The orthopedic surgeon and all other testSubjective outcomes ing staff were blinded to the randomization of groups to limit any bias, altered treatment, or encouragement during testing procedures." However, participants were not blinded

Blinding of participants and personnel Low risk (performance bias)

Objective outcomes

Blinding of outcome assessment (detection High risk bias)

Subjective outcomes
"The orthopedic surgeon and all other testing staff were blinded to the randomization of groups to limit any bias, altered treatment, or encouragement during testing procedures"

Participants were not blinded 


\section{Magnus 2013 (Continued)}

\begin{tabular}{l|l}
$\begin{array}{l}\text { Blinding of outcome assessment (detection } \\
\text { bias) }\end{array}$ & Low risk \\
$\begin{array}{l}\text { Objective outcomes (functional impair- } \\
\text { ment) }\end{array}$ & $\begin{array}{l}\text { "The orthopedic surgeon and all other test- } \\
\text { ing staff were blinded to the randomiza- } \\
\text { tion of groups to limit any bias, altered } \\
\text { treatment, or encouragement during test- } \\
\text { ing procedures" }\end{array}$ \\
\hline
\end{tabular}

Blinding of outcome assessment (detection Low risk bias)

Only outcome reported was number of participants attending physiotherapy

Complications, number of sessions, return

to former activity

\begin{tabular}{|c|c|c|}
\hline $\begin{array}{l}\text { Incomplete outcome data (attrition bias) } \\
\text { Short term follow-up (up to } 3 \text { months) }\end{array}$ & High risk & $\begin{array}{l}\text { Difference between the two groups in par- } \\
\text { ticipants missing from follow-up ( } 9 \text { versus } \\
\text { 3) and also missing data points for remain- } \\
\text { ing participants }\end{array}$ \\
\hline $\begin{array}{l}\text { Incomplete outcome data (attrition bias) } \\
\text { Longer term follow-up }\end{array}$ & High risk & $\begin{array}{l}\text { Difference between the two groups in par- } \\
\text { ticipants missing from follow-up ( } 9 \text { versus } \\
\text { 3) and also missing data points for remain- } \\
\text { ing participants }\end{array}$ \\
\hline Selective reporting (reporting bias) & Unclear risk & $\begin{array}{l}\text { Protocol and trial registration document } \\
\text { unavailable. Some reference made to post- } \\
\text { hoc analysis }\end{array}$ \\
\hline Major baseline imbalance bias? & Unclear risk & $\begin{array}{l}\text { No data on injury severity but imbalance in } \\
\text { numbers operated on ( } 9 \text { versus } 2) \text {. Age and } \\
\text { hand dominance similar, but actual injured } \\
\text { hand not stated }\end{array}$ \\
\hline $\begin{array}{l}\text { Other performance bias (e.g. differential } \\
\text { expertise bias) }\end{array}$ & Unclear risk & $\begin{array}{l}\text { Although measures, such as regular tele- } \\
\text { phone calls to the control group, taken to } \\
\text { ensure comparability, more fractures were } \\
\text { operated on in the intervention group: } 9 \\
\text { versus } 2 \text {; in analysis: } 6 \text { versus } 2 \text {. The same } \\
\text { numbers in each group ( } 7 \text { versus } 7 \text { ) at- } \\
\text { tended physiotherapy }\end{array}$ \\
\hline
\end{tabular}

Pasila 1974

\begin{tabular}{ll}
\hline Methods & Method of randomisation: “random sample" at hospital admission \\
& Assessor blinding: no \\
& Intention-to-treat analysis: no \\
& Loss to follow-up: 39 \\
\hline Participants & University Central Hospital, Helsinki, Finland \\
& 135 participants; data for 96 provided \\
& Inclusion criteria: Colles' fracture, displaced "typical radial fracture", aged 16 to 65 years
\end{tabular}




$\begin{array}{ll} & \text { Exclusion criteria: see above } \\ \text { Classification: Older } \\ \text { Sex: (of 96) } 89 \text { female } 93 \%) \\ \text { Age: < } 40 \text { years: } 67 ; 40 \text { to } 60 \text { years: } 20 ;>60 \text { years: } 9 \\ \text { Assigned: ?/? [physiotherapy/control] } \\ \text { Assessed: } 48 / 48 \text { (probably; at } 12 \text { weeks) }\end{array}$

Funding, ethics and patient consent

Notes
No mention of funding source, ethics or informed patient consent

Request for further information sent 1 August 2001. However, last publication of Pasila identified in 1982 and envelope returned stamped "Unknown"

Means and standard deviations for functional impairments were extracted from the graphs in the trial report

\section{Risk of bias}

Bias

Random sequence generation (selection Unclear risk bias)

\section{Authors' judgement}

\section{Support for judgement}

Insufficient details to judge

Report: "By random sample the patients were classified in two groups on arrival at the hospital"

\section{Allocation concealment (selection bias) Unclear risk}

Blinding of participants and personnel High risk (performance bias)

Subjective outcomes

\section{As above}

Insufficient details to judge

No blinding for the sole outcome ('subjective attitude') in this category 
Pasila 1974 (Continued)

\begin{tabular}{l|l}
$\begin{array}{l}\text { Blinding of participants and personnel } \\
\text { (performance bias) } \\
\text { Objective outcomes }\end{array}$ & $\begin{array}{l}\text { No blinding. Some control over delivery of } \\
\text { interventions but insufficient description } \\
\text { of safeguards }\end{array}$ \\
\hline
\end{tabular}

Blinding of outcome assessment (detection High risk

bias)

No blinding for the sole outcome ('subjective attitude') in this category

Subjective outcomes

Blinding of outcome assessment (detection High risk bias)

Objective outcomes (functional impairment)
No blinding, even though indication of a systematic approach to outcome assessment

No blinding but detection bias less likely for these outcomes
Blinding of outcome assessment (detection Unclear risk bias)

Complications, number of sessions, return

to former activity

Incomplete outcome data (attrition bias) High risk

Short term follow-up (up to 3 months)

\begin{tabular}{|c|c|c|}
\hline $\begin{array}{l}\text { Incomplete outcome data (attrition bias) } \\
\text { Longer term follow-up }\end{array}$ & Unclear risk & Short-term outcomes only \\
\hline Selective reporting (reporting bias) & High risk & $\begin{array}{l}\text { No protocol available. Some recorded out- } \\
\text { comes not reported split by treatment or at } \\
\text { all }\end{array}$ \\
\hline Major baseline imbalance bias? & Unclear risk & Baseline data not available \\
\hline $\begin{array}{l}\text { Other performance bias (e.g. differential } \\
\text { expertise bias) }\end{array}$ & Low risk & $\begin{array}{l}\text { Routinely performed interventions. Care } \\
\text { programmes clearly identical }\end{array}$ \\
\hline
\end{tabular}

\section{Rozencwaig 1996}

$\begin{array}{ll}\text { Methods } & \text { Quasi-randomised: alternation or odd and even clinic numbers } \\ \text { Assessor blinding: unlikely } \\ \text { Intention-to-treat analysis: likely } \\ \text { Loss to follow-up: none }\end{array}$


Assigned: 3/4 [continuous passive motion/control]

Assessed: 3/4 (recovery)

\begin{tabular}{ll} 
Interventions & $\begin{array}{l}\text { Timing of intervention: after external fixation (lasting } 6 \text { to } 8 \text { weeks) } \\
\text { All participants had "traditional occupational therapy" consisting of heat modalities, } \\
\text { active-assisted ROM, mobilisation, passive ROM, progressing to strengthening when } \\
\text { appropriate (avoiding excessive force), in an outpatient setting } \\
\text { 1. Continuous passive motion (CPM). Therapist instructed participants on the use of } \\
\text { CPM. Probably: CPM device use } 4 \text { to } 6 \text { hours per day over } 1 \text { month } \\
\text { 2. Control: occupational therapy only }\end{array}$ \\
\hline Outcomes & $\begin{array}{l}\text { Length of follow-up: until recovery } \\
\text { 1. Functional: functional evaluation score (0: dependent to 7: independent), ROM }\end{array}$ \\
\hline Funding, ethics and patient consent & \begin{tabular}{l} 
No mention of funding source, ethics or informed patient consent \\
\hline Notes
\end{tabular} \\
$\begin{array}{l}\text { Only reported in a conference proceedings abstract. Further details received from Dr } \\
\text { Richard Rozencwaig (29 August \& } 4 \text { September 2001). Also, from Susan Fortier (17 } \\
\text { October 2001) in association with Dr Jefferson Kaye). Confirmation of no other publi- } \\
\text { cation past or forthcoming; no further details available }\end{array}$
\end{tabular}

Risk of bias

\begin{tabular}{|c|c|c|}
\hline Bias & Authors' judgement & Support for judgement \\
\hline $\begin{array}{l}\text { Random sequence generation (selection } \\
\text { bias) }\end{array}$ & High risk & $\begin{array}{l}\text { Quasi-randomised } \\
\text { Email (20/08/2001): "The randomization } \\
\text { technique consisted of alternating between } \\
\text { the two treatment groups" } \\
\text { Letter (17/10/2001): "method of random- } \\
\text { ization was odd and even clinic \#'s" }\end{array}$ \\
\hline Allocation concealment (selection bias) & High risk & As above \\
\hline
\end{tabular}

Blinding of participants and personnel Unclear risk

No patient-reported outcomes

(performance bias)

Subjective outcomes

Blinding of participants and personnel High risk (performance bias)

Objective outcomes

No blinding

Blinding of outcome assessment (detection Unclear risk

No patient-reported outcomes

bias)

Subjective outcomes

Blinding of outcome assessment (detection High risk

bias)

Objective outcomes (functional impair-

Rehabilitation for distal radial fractures in adults (Review)

Copyright @ 2015 The Cochrane Collaboration. Published by John Wiley \& Sons, Ltd. 
Rozencwaig 1996 (Continued)

ment)

\begin{tabular}{|c|c|c|}
\hline $\begin{array}{l}\text { Blinding of outcome assessment (detection } \\
\text { bias) } \\
\text { Complications, number of sessions, return } \\
\text { to former activity }\end{array}$ & High risk & No indication of assessor blinding \\
\hline $\begin{array}{l}\text { Incomplete outcome data (attrition bias) } \\
\text { Short term follow-up (up to } 3 \text { months) }\end{array}$ & Unclear risk & $\begin{array}{l}\text { Probably none lost to follow-up and inten- } \\
\text { tion-to-treat analysis but the follow-up for } \\
\text { the trial stopped on achievement of func- } \\
\text { tional 'independence'. The } 7 \text { patients were } \\
\text { followed up clinically for longer }\end{array}$ \\
\hline $\begin{array}{l}\text { Incomplete outcome data (attrition bias) } \\
\text { Longer term follow-up }\end{array}$ & Unclear risk & Short-term outcomes only \\
\hline Selective reporting (reporting bias) & High risk & No protocol, minimally reported trial \\
\hline Major baseline imbalance bias? & Unclear risk & No baseline data \\
\hline $\begin{array}{l}\text { Other performance bias (e.g. differential } \\
\text { expertise bias) }\end{array}$ & Unclear risk & No information \\
\hline
\end{tabular}

\section{Souer 2011}

\begin{tabular}{|c|c|}
\hline Methods & $\begin{array}{l}\text { Method of randomisation: computer random number generator } \\
\text { Assessor blinding: no, but independent and uninformed therapists } \\
\text { Intention-to-treat analysis: no, post-randomisation losses, } 1 \text { unexplained protocol vio- } \\
\text { lation } \\
\text { Loss to follow-up: } 18 \text { (at } 6 \text { months) }\end{array}$ \\
\hline Participants & $\begin{array}{l}\text { Orthopaedic Hand and Upper Extremity Service, Massachusetts General Hospital, } \\
\text { Boston, Massachusetts, USA } \\
94 \text { participants } \\
\text { Inclusion criteria: unstable distal radius fracture treated with a volar locking plate and } \\
\text { screws only within } 4 \text { weeks of injury, age } 18+\text { years. } \\
\text { Exclusion criteria: other injury, complex distal radial fracture treated with alternative or } \\
\text { ancillary fixation, patients not planning to return for follow-up, dependency for basic } \\
\text { functioning } \\
\text { Classification: AO (A, B and C) } \\
\text { Sex: } 61 \text { female (65\%) } \\
\text { Age: mean } 50 \text { years } \\
\text { Assigned: } 46 / 48 \text { [occupational therapy / home exercise] } \\
\text { Assessed: } 37 / 39 \text { (at } 6 \text { months) }\end{array}$ \\
\hline Interventions & $\begin{array}{l}\text { Timing of intervention: Surgery was on average } 8 \text { days after injury. Allocation after the } \\
\text { first preoperative visit in the surgeon's office } \\
\text { 1. Occupational therapy: Formal occupational therapy with supervised exercises to regain }\end{array}$ \\
\hline
\end{tabular}


digit, wrist, and forearm motion and to strengthen the hand. Consistent with "usual practice", the content, frequency, and duration of the rehabilitation programme were at the discretion of the treating hand therapist

2. Instruction for home exercises. Participants were provided with wrist splint and instructions and coaching for independent exercises to perform at home on their own. Participants were instructed to wear the splint until they had full finger and forearm motion, then to wean themselves off the splint and on to wrist flexion and extension. Participants were advised to perform exercises as often as possible, but at least three to four times a day for a minimum of 30 minutes. There was no formal strengthening programme. Participants were guided to "an athletic so-called healthy stretch mindset, in which the pain of the stretch was seen as a part of recovery"

Outcomes

Length of follow-up: 6 months, also 3 months and 6 weeks (not reported)

1. Functional: DASH, Mayo wrist scores, Gartland and Werley score (not used in review) , pain at rest (VAS), grip strength, pinch strength, ROM (wrist flexion and extension arc, extension, flexion, radial deviation, ulnar deviation, supination, pronation);

2. Complications (primarily surgery related);

3. Others: change of treatment, also radiological outcomes (volar angulation, ulnar inclination, ulnar variance) not reported in this review, request for change of treatment

Funding, ethics and patient consent

Study protocol approved by Human Research Committee at institution. Mention of funding support under 'Disclosure', plus mention of non-specified conflict of interest. Informed consent

Notes

Risk of bias

Bias

Authors' judgement

Support for judgement

Random sequence generation (selection Low risk bias)

"patients were randomized equally to either the occupational therapy or independent exercise cohort according to a computerized random-number generator". Enrolment occurred in surgeon's office

\begin{tabular}{|c|c|c|}
\hline Allocation concealment (selection bias) & Unclear risk & No details \\
\hline $\begin{array}{l}\text { Blinding of participants and personnel } \\
\text { (performance bias) } \\
\text { Subjective outcomes }\end{array}$ & High risk & $\begin{array}{l}\text { "therapist was not involved in the study de- } \\
\text { sign, and therapists were not informed that } \\
\text { patients were in a study". Participants were } \\
\text { not blinded }\end{array}$ \\
\hline
\end{tabular}

Blinding of participants and personnel Unclear risk (performance bias)

Objective outcomes

"therapist was not involved in the study design, and therapists were not informed that patients were in a study"

Rehabilitation for distal radial fractures in adults (Review) 


\section{Souer 2011 (Continued)}

\begin{tabular}{|c|c|c|}
\hline $\begin{array}{l}\text { Blinding of outcome assessment (detection } \\
\text { bias) } \\
\text { Subjective outcomes }\end{array}$ & High risk & $\begin{array}{l}\text { "All patients were examined by a trained } \\
\text { research assistant who was not involved in } \\
\text { the care of the patient but was not blinded } \\
\text { to assignment" Participants were not blind }\end{array}$ \\
\hline $\begin{array}{l}\text { Blinding of outcome assessment (detection } \\
\text { bias) } \\
\text { Objective outcomes (functional impair- } \\
\text { ment) }\end{array}$ & Unclear risk & $\begin{array}{l}\text { "All patients were examined by a trained } \\
\text { research assistant who was not involved in } \\
\text { the care of the patient but was not blinded } \\
\text { to assignment" }\end{array}$ \\
\hline
\end{tabular}

Blinding of outcome assessment (detection Low risk bias)

Only complications (of injury/surgery) re-

Complications, number of sessions, return

to former activity

Incomplete outcome data (attrition bias) Low risk Short term follow-up (up to 3 months)

Clear description of losses, which were few: $3 / 46(6.5 \%)$ versus $1 / 48(2.1 \%)$

Incomplete outcome data (attrition bias) Unclear risk

Clear description of losses, with 9 in each Longer term follow-up group (20\% versus $19 \%)$

Selective reporting (reporting bias) Low risk

Mention of protocol and also trial registration refers to same outcomes (as reported)

Major baseline imbalance bias? Low risk

"The two cohorts were comparable ...." Also evident from table of baseline characteristics

Other performance bias (e.g. differential Unclear risk expertise bias)

Lack of clarity regarding occupational therapy means this is unclear

Svensson 1993

Methods

Participants
Method of randomisation: involved sealed envelopes

Assessor blinding: no

Intention-to-treat analysis: no, 3 participants excluded

Loss to follow-up: 9 (+3 excluded)

Bispebjerg Hospital, Copenhagen, Denmark

43 participants

Inclusion criteria: Colles' fracture, age 55+ years, female, referred to rheumatological ward for rehabilitation of hand function after plaster cast removal. Consent

Exclusion criteria: previous fracture of same forearm/hand, reflex dystrophy, ipsilateral hemiparesis or other neurological disease, infectious skin disease, disfiguring rheumatic disease

Classification: Frykman

Sex: all female (100\%)

Age: (of 40) median 72 years; range 55 to 90 years 
Timing of intervention: following plaster cast removal; first occupational therapy treatment median 25 days, range 1 to 46 days

All participants had occupational therapy thrice weekly for 3 weeks followed by further treatment as required. Instruction for home exercises for daily practice given on first session. Approximately 1 hour sessions involved limber-up in tepid water 10 minutes, venous pump exercise (elevated arm), range of motion, grip strength, pinch exercises. Guidance for ADL

1. 20 minutes of intermittent pneumatic compression before OT session. Flowtron Air. Continuously variable pressure 30 to $70 \mathrm{mmHg}$, cycle time 2 minutes

2. Control: no compression

Outcomes

Length of follow-up: 3 months; also 3 weeks.

1. Functional: use of hand in daily skills (VAS), pain at rest or during function (VAS) , grip strength, ROM (pronation; supination; flexion; extension; radial deviation; ulnar deviation; finger abduction, thumb opposition)

2. Clinical: oedema. Complications: no mention

3. Number of sessions. Patient satisfaction

Funding, ethics and patient consent

No mention of funding

Mention of the ethics committee (and Helsinki Declaration) and informed patient consent

Notes

Incomplete translation from Danish by Kirsten Lone Jensen

Request for further information sent 30 August 2001

\section{Risk of bias}

\begin{tabular}{|c|c|c|}
\hline Bias & Authors' judgement & Support for judgement \\
\hline $\begin{array}{l}\text { Random sequence generation (selection } \\
\text { bias) }\end{array}$ & Unclear risk & $\begin{array}{l}\text { Involved sealed envelopes. No mention of } \\
\text { method of random sequence generation }\end{array}$ \\
\hline Allocation concealment (selection bias) & Unclear risk & $\begin{array}{l}\text { Involved sealed envelopes - no mention of } \\
\text { safeguards }\end{array}$ \\
\hline $\begin{array}{l}\text { Blinding of participants and personnel } \\
\text { (performance bias) } \\
\text { Subjective outcomes }\end{array}$ & High risk & Participants and personnel not blinded \\
\hline $\begin{array}{l}\text { Blinding of participants and personnel } \\
\text { (performance bias) } \\
\text { Objective outcomes }\end{array}$ & High risk & Participants and personnel not blinded \\
\hline $\begin{array}{l}\text { Blinding of outcome assessment (detection } \\
\text { bias) } \\
\text { Subjective outcomes }\end{array}$ & High risk & $\begin{array}{l}\text { Patient examinations were performed by } \\
\text { the treating occupational therapist }\end{array}$ \\
\hline
\end{tabular}

Rehabilitation for distal radial fractures in adults (Review) 


\section{Svensson 1993 (Continued)}

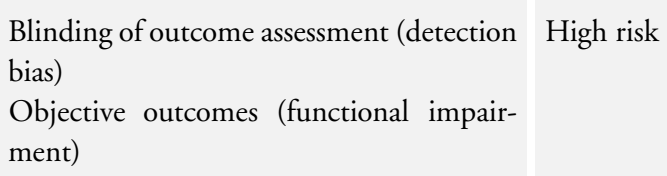

\begin{tabular}{|c|c|c|}
\hline $\begin{array}{l}\text { Incomplete outcome data (attrition bias) } \\
\text { Short term follow-up (up to } 3 \text { months) }\end{array}$ & High risk & $\begin{array}{l}\text { Three exclusions and a further } 9 \text { patients } \\
\text { missing at } 3 \text { months (subjective outcomes) } \\
\text { - numbers in each group not available at } 3 \\
\text { months. Complications not split by groups }\end{array}$ \\
\hline $\begin{array}{l}\text { Incomplete outcome data (attrition bias) } \\
\text { Longer term follow-up }\end{array}$ & Unclear risk & No longer-term outcomes \\
\hline Selective reporting (reporting bias) & Unclear risk & $\begin{array}{l}\text { No protocol available but one submitted to } \\
\text { Ethics committee }\end{array}$ \\
\hline Major baseline imbalance bias? & Unclear risk & $\begin{array}{l}\text { Reported to be comparable for } 40 \text { of the } 43 \\
\text { in trial. No data }\end{array}$ \\
\hline $\begin{array}{l}\text { Other performance bias (e.g. differential } \\
\text { expertise bias) }\end{array}$ & Low risk & $\begin{array}{l}\text { Adjunctive therapy on top of standard re- } \\
\text { habilitation }\end{array}$ \\
\hline
\end{tabular}

\section{Taylor 1994}

\begin{tabular}{|c|c|}
\hline Methods & $\begin{array}{l}\text { Method of randomisation: coin toss for first patient of every pair, second patient allocated } \\
\text { to other group } \\
\text { Assessor blinding: no } \\
\text { Intention-to-treat analysis: likely } \\
\text { Loss to follow-up: none }\end{array}$ \\
\hline Participants & $\begin{array}{l}\text { Box Hill Hospital, Victoria, Australia } \\
30 \text { participants } \\
\text { Inclusion criteria: Colles' fracture, treated with plaster cast. } \\
\text { Exclusion criteria: < } 35 \text { years, multiple concurrent upper limb fracture } \\
\text { Classification: not stated } \\
\text { Sex: } 24 \text { female }(80 \%) \\
\text { Age: mean } 63 \text { years; range } 39 \text { to } 78 \text { years } \\
\text { Assigned: } 15 / 15 \text { [passive mobilisation/massage] } \\
\text { Assessed: } 15 / 15 \text { (at discharge) }\end{array}$ \\
\hline Interventions & $\begin{array}{l}\text { Timing of intervention: within } 3 \text { working days following plaster cast removal ( } 6 \text { weeks } \\
\text { immobilisation) } \\
\text { All participants had twice weekly treatment at physiotherapy department by experienced }\end{array}$ \\
\hline
\end{tabular}


Taylor 1994 (Continued)

orthopaedic physiotherapists. All received standard regimen of heat (wax or hot pack), active exercise (exercise card for home use - patients taught free, stretch and strengthening exercises, and supervised at each treatment session) and home advice (use of affected arm for ADL: but avoid excessive force). Discharge at discretion of physiotherapists acceptable ROM/function or no further benefit expected. All 4 physiotherapists had attended a course on passive mobilisation

1. Passive mobilisation for up to 5 minutes

2. Sham: 5 minutes of soft tissue massage

Outcomes

Length of follow-up: until discharge (mean 26 days)

1. Functional: wrist extension

2. Number of sessions and time until discharge

Funding, ethics and patient consent

No mention of funding source, ethics or informed patient consent

Notes

Further details of trial received from Dr Nick Taylor 26 and 27 July 2001

Risk of bias

Bias

Authors' judgement

Support for judgement

Random sequence generation (selection Unclear risk bias)

From email: "The method of randomisation was for the investigator (NFT) to toss a coin for the first subject to allocate group. The second subject was then allocated to the other group. The coin was then tossed to allocate the third subject and the fourth subject was allocated to the other group and so on, ensuring equal numbers in each group"

Allocation concealment (selection bias) High risk

From email: "Allocation was not concealed at the time of randomisation"

Blinding of participants and personnel Unclear risk

No subjective outcomes

(performance bias)

Subjective outcomes

Blinding of participants and personnel High risk

(performance bias)

Objective outcomes

The physiotherapists providing the treatment were not blinded. Participants may have been blinded - use of sham control

Blinding of outcome assessment (detection Unclear risk

No subjective outcomes

bias)

Subjective outcomes

Blinding of outcome assessment (detection High risk bias)

Objective outcomes (functional impair-

No blinding of outcome assessors, who were those providing the intervention

Rehabilitation for distal radial fractures in adults (Review)

Copyright @ 2015 The Cochrane Collaboration. Published by John Wiley \& Sons, Ltd. 
Taylor 1994 (Continued)

ment)

Blinding of outcome assessment (detection High risk

bias)

Complications, number of sessions, return

to former activity
No blinding of outcome assessors, who were those providing the intervention

Incomplete outcome data (attrition bias) Low risk

Short term follow-up (up to 3 months)

\begin{tabular}{|c|c|c|}
\hline $\begin{array}{l}\text { Incomplete outcome data (attrition bias) } \\
\text { Longer term follow-up }\end{array}$ & Unclear risk & No longer-term follow-up \\
\hline Selective reporting (reporting bias) & Unclear risk & $\begin{array}{l}\text { No protocol available but efforts made to } \\
\text { standardise outcome measurement }\end{array}$ \\
\hline Major baseline imbalance bias? & Unclear risk & Only age and gender data provided \\
\hline $\begin{array}{l}\text { Other performance bias (e.g. differential } \\
\text { expertise bias) }\end{array}$ & Low risk & $\begin{array}{l}\text { Comparable care programmes. None of the } \\
\text { physiotherapists had postgraduate qualifi- } \\
\text { cations in intervention but additional anal- } \\
\text { yses in the trial report showed the therapist } \\
\text { had equivalent general skills }\end{array}$ \\
\hline
\end{tabular}

Toomey 1986

Methods

Participants
Method of randomisation: not stated

Assessor blinding: yes

Intention-to-treat analysis: likely but some participants may have been excluded

Loss to follow-up: probably none

\begin{tabular}{ll}
\hline Participants & Montreal General Hospital, Canada \\
24(?) participants \\
Inclusion criteria: Colles' fracture, treated with plaster cast immobilisation referred to \\
Physical Medicine Department. \\
Exclusion criteria: associated fractures or conditions such as shoulder-hand syndrome, \\
rheumatoid arthritis, brachial plexus injuries. Occupational therapy for involved hand. \\
No consent \\
Classification: own: undisplaced/displaced/ulna fracture/comminuted \\
Sex: 20 female $(83 \%)$ \\
Age: mean 60 years; range 40 to 80 years \\
Assigned: $12 / 12$ (probably) [whirlpool / towel] \\
Assessed: $12 / 12$ (by end of treatment, 6 weeks maximum) \\
\hline
\end{tabular}

Interventions

Timing of intervention: on average 6 days following plaster cast removal (mean 6 weeks immobilisation)

All scheduled for 12 sessions, twice weekly, lasting 45 minutes each of physiotherapy. (No occupational therapy was given.) Each session, after the trial interventions (see below), 
participants received massage, joint mobilisation, active and resistive exercises

1. Whirlpool. Seated participants had hand, wrist and forearm in whirlpool at room temperature for first 15 minutes of each session

2. Towel. Seated participants had hand and wrist in two standard hospital towels for first 15 minutes of each session

Outcomes
Length of follow-up: until discharge (maximum 6 weeks)

1. Functional: grip strength, pain, ROM (pronation; supination; flexion; extension; radial deviation; ulnar deviation; finger flexion)

2. Clinical: hand volume

No mention of funding source or ethics

All signed an informed consent form prior to entering the trial

Notes

Report indicated that if patient's condition did not improve and an alternative treatment was warranted or if it worsened, then he/she was removed from the trial. No details are given of whether this happened

Request for further information sent 8 August 2001

\section{Risk of bias}

\begin{tabular}{|c|c|c|}
\hline Bias & Authors' judgement & Support for judgement \\
\hline $\begin{array}{l}\text { Random sequence generation (selection } \\
\text { bias) }\end{array}$ & Unclear risk & $\begin{array}{l}\text { "The subjects were randomly assigned to } \\
\text { one of two treatment groups" }\end{array}$ \\
\hline Allocation concealment (selection bias) & Unclear risk & As above \\
\hline $\begin{array}{l}\text { Blinding of participants and personnel } \\
\text { (performance bias) } \\
\text { Subjective outcomes }\end{array}$ & High risk & No blinding (pain) \\
\hline
\end{tabular}

Blinding of participants and personnel High risk (performance bias)

Objective outcomes
No blinding

Pain: patients would have been aware of their treatment group
Blinding of outcome assessment (detection High risk bias)

Subjective outcomes
Blinding of outcome assessment (detection Low risk bias)

Objective outcomes (functional impairment)
"Assessments by independent evaluators (who were unaware of the patient's group assignment) were performed prior to and following each whirlpool or towel treatment. Due to the obvious signs of whirlpool therapy (redness and wrinkling of skin), the subjects of both groups were asked to wait an additional five minutes before returning to the evaluator to be re- 
Toomey 1986 (Continued)

assessed"

\begin{tabular}{|c|c|c|}
\hline & & assessed" \\
\hline $\begin{array}{l}\text { Blinding of outcome assessment (detection } \\
\text { bias) } \\
\text { Complications, number of sessions, return } \\
\text { to former activity }\end{array}$ & Unclear risk & Ditto (but outcomes not reported) \\
\hline $\begin{array}{l}\text { Incomplete outcome data (attrition bias) } \\
\text { Short term follow-up (up to } 3 \text { months) }\end{array}$ & Unclear risk & $\begin{array}{l}\text { It was not clear if there were any post- } \\
\text { randomisation exclusions. "If the patients } \\
\text { did not improve and an alternate or addi- } \\
\text { tional form of therapy was warranted, or if } \\
\text { the patient worsened, he/she was removed } \\
\text { from the trial and referred to an appropri- } \\
\text { ate source of treatment" }\end{array}$ \\
\hline $\begin{array}{l}\text { Incomplete outcome data (attrition bias) } \\
\text { Longer term follow-up }\end{array}$ & Unclear risk & No longer-term follow-up \\
\hline Selective reporting (reporting bias) & Unclear risk & $\begin{array}{l}\text { Indication of a systematic approach but no } \\
\text { protocol or trial registration available }\end{array}$ \\
\hline Major baseline imbalance bias? & Low risk & No major imbalance \\
\hline $\begin{array}{l}\text { Other performance bias (e.g. differential } \\
\text { expertise bias) }\end{array}$ & Low risk & Clearly comparable care programmes \\
\hline
\end{tabular}

Wakefield 2000

\begin{tabular}{ll} 
Methods & $\begin{array}{l}\text { Method of randomisation: numbered sealed envelopes opened at fracture clinic; use of } \\
\text { random numbers generated using computer programme in blocks of } 10 \text { by independent } \\
\text { colleague } \\
\text { Assessor blinding: yes for objective measures ROM and grip strength } \\
\text { Intention-to-treat analysis: claimed but decided to follow up only } 66 \text { participants to } 6 \\
\text { months } \\
\text { Loss to follow-up: } 6 \text { (at } 3 \text { months) }\end{array}$ \\
\hline From Edinburgh Royal infirmary, UK \\
\hline $\begin{array}{l}96 \text { participants } \\
\text { Inclusion criteria: radiologically confirmed distal radial fracture, treated with plaster } \\
\text { immobilisation, attending outpatients, age over } 55 \text { years, informed consent. }\end{array}$ \\
$\begin{array}{l}\text { Exclusion criteria: mental test score }<8 \text {, participation in another clinical trial, bilateral } \\
\text { wrist fractures, previous fracture of unaffected wrist, surgical treatment of wrist, clinical }\end{array}$ \\
signs of CRPS-1 at time of plaster cast removal \\
$\begin{array}{l}\text { Classification: AO } \\
\text { Sex: } 87 \text { female }(91 \%)\end{array}$ \\
$\begin{array}{l}\text { Age: mean } 73 \text { years; range } 55 \text { to } 90 \text { years } \\
\text { Assigned: } 49 / 47 \text { [physiotherapy / control] } \\
\text { Assessed: } 47 / 43 \text { (at } 3 \text { months); } 34 / 32 \text { (at } 6 \text { months) }\end{array}$ \\
\hline
\end{tabular}

Rehabilitation for distal radial fractures in adults (Review) 
Wakefield 2000 (Continued)

Interventions
$\begin{aligned} & \text { Timing of intervention: following plaster cast removal (and on average } 37 \text { days immobil- } \\ & \text { isation). All participants were taught home exercises by the physiotherapist at the fracture } \\ & \text { clinic }\end{aligned}$
$\begin{aligned} & \text { 1. Referral for routine physiotherapy at participant's local hospital/clinic (there were } 4 \\ & \text { hospitals and } 11 \text { health clinics). Contents of treatment at discretion of therapists (all } \\ & \text { were qualified state registered physiotherapists); these involved different combinations } \\ & \text { of active exercises, passive accessory movements and stretches, and strengthening and } \\ & \text { functional exercises } \\ & \text { 2. Home exercises as taught at outpatients only }\end{aligned}$

Outcomes

Length of follow-up: 6 months (from fracture); also 3 months

1. Functional: grip strength, ROM (pronation-supination; flexion-extension, radial-ulnar deviation); functional score relating to ADL (Sheehan 1983); pain; QOL (physical \& mental health SF-36, UK version); total outcome score (from grip, ROM and functional score). Control group participants requiring physiotherapy

2. Number of physiotherapy attendances

3. Complications (no information)

Funding, ethics and patient consent

No mention of funding source

Mention of the ethics committee and obtaining of informed consent prior to trial enrolment

Notes

Reason given for reduction in numbers at last follow-up: "Preliminary analysis indicated that sufficient numbers of patients had been recruited and therefore only 66 were followed up at six months"

Further details of trial received from Mrs Alison Wakefield 10 September 2001

\section{Risk of bias}

\begin{tabular}{lll}
\hline Bias Authors' judgement $\quad$ Support for judgement
\end{tabular}

Random sequence generation (selection Low risk bias)
Low risk
By letter (dated: 6 September 2001): "Block randomisation was used - random numbers were generated on an excel spreadsheet by a colleague in blocks of 10 "

By letter (dated: 6 September 2001), continued from above: "The colleague then divided the patients into physiotherapy and home exercise groups using these numbers, put the allocations into sealed envelopes and numbered the envelopes accordingly. The allocations were kept securely so that myself, (the observer) and physiotherapist in the Fracture clinic were blind as to the treatment each patient would receive. After informed consent was obtained the patients were sent to the physiotherapist in the 
Wakefield 2000 (Continued)

Fracture clinic who held the envelopes, she opened them and referred them to physiotherapy or not depending on the contents of the envelope. Therefore allocation was concealed from the observer at the time of randomisation for all patients concerned with the trial"

Blinding of participants and personnel High risk

Neither participants nor personnel provid(performance bias)

ing care were blinded

Subjective outcomes

Blinding of participants and personnel High risk (performance bias)

Neither participants nor personnel providing care were blinded

Objective outcomes

Blinding of outcome assessment (detection High risk

The participants were not blinded

bias)

Subjective outcomes

Blinding of outcome assessment (detection Unclear risk

bias)

Objective outcomes (functional impair-

ment)

At trial entry: "The observer (AW) was blinded as to which treatment each patient entered: this reduced observer bias at future assessments." However, there is no mention of other actions to safeguard blinding

Blinding of outcome assessment (detection Low risk bias)

While insufficiently reported, these out-

Complications, number of sessions, return comes are less susceptible to bias

to former activity

Incomplete outcome data (attrition bias) Unclear risk Short term follow-up (up to 3 months)

Loss in follow-up at 3 months balanced between 2 groups (2 versus 4). While claim of "intention-to-treat analysis", denominators not reported for continuous data

Incomplete outcome data (attrition bias) High risk

Longer term follow-up

Based on a "preliminary analysis", only 66 patients $(69 \%)$ were followed up at six months

Selective reporting (reporting bias) High risk

No protocol and some evidence of posthoc decisions

Major baseline imbalance bias? Low risk

No concerns

Other performance bias (e.g. differential Low risk expertise bias)

While there was a lack of information, there was a background of 'usual treatment', with regular follow-ups

Rehabilitation for distal radial fractures in adults (Review)

Copyright @ 2015 The Cochrane Collaboration. Published by John Wiley \& Sons, Ltd. 
Methods

Method of randomisation: random number tables, sealed envelopes opened by orthopaedic surgeon

Assessor blinding: yes for ROM and grip strength

Intention-to-treat analysis: no, 2 participants excluded

Loss to follow-up: none

Box Hill Hospital, Victoria, Australia
18 participants
Inclusion criteria: Colles' fracture, treated with plaster cast, attending outpatients, no
"significant past history".
Exclusion criteria: see above.
Classification: Frykman
Sex: 17 female $(94 \%)$
Age: mean 76 years
Assigned: $9 / 9$ [physiotherapy / control]
Assessed: $8 / 8$ (at 6 weeks)

Interventions

Timing of intervention: following plaster cast removal (and on average 43 days immobilisation)

1. Referral for routine physiotherapy at physiotherapy department. Contents of treatment at discretion of hospital therapists, always included active exercises including home exercise programme, advice and, for $47 \%$ of all treatments, passive joint mobilisation

2. Home exercise sheet and simple home instructions given at outpatients by orthopaedic surgeon/registrar

Length of follow-up: 6 weeks
$\begin{aligned} & \text { 1. Functional: grip strength, wrist extension } \\ & \text { 2. Number of physiotherapy attendances of intervention group } \\ & \text { 3. Non-compliance }\end{aligned}$

Funding, ethics and patient consent

No mention of funding source

Mention of approval by hospital and university ethics committees and that all "subjects gave informed consent"

Notes

Further details of trial received from Dr Nick Taylor 27 July 2001

Risk of bias

\begin{tabular}{|c|c|c|}
\hline Bias & Authors' judgement & Support for judgement \\
\hline $\begin{array}{l}\text { Random sequence generation (selection } \\
\text { bias) }\end{array}$ & Low risk & $\begin{array}{l}\text { From email ( } 27 \text { July } 2001) \text { : "The sealed en- } \\
\text { velopes were prepared by one of the inves- } \\
\text { tigators (NFT) prior to the study using a } \\
\text { table of random numbers" }\end{array}$ \\
\hline Allocation concealment (selection bias) & Low risk & $\begin{array}{l}\text { From email ( } 27 \text { July 2001): "The ran- } \\
\text { dom allocation [was] conducted by the } \\
\text { orthopaedic surgeon or registrar, whom } \\
\text { opened a sealed envelope indicating either }\end{array}$ \\
\hline
\end{tabular}


Watt 2000 (Continued)

"physiotherapy" or "non-physiotherapy". Therefore allocation was concealed at the time of randomisation"

Blinding of participants and personnel Unclear risk

No subjective outcomes

(performance bias)

Subjective outcomes

Blinding of participants and personnel High risk

(performance bias)

Neither the physiotherapist(s) providing

Objective outcomes the treatment nor the participants were blinded

Blinding of outcome assessment (detection Unclear risk bias)

Subjective outcomes

Blinding of outcome assessment (detection Low risk

bias)

Objective outcomes (functional impair-

ment)
No subjective outcomes

From email (27 July 2001): “The investigator who took the measurements (CFW) was blind to group allocation.... To ensure the investigator remained blinded to group allocation, he left the room before the orthopaedic surgeon or registrar opened. " "To safeguard that the investigator remained blinded at review, the investigator did not attend the physiotherapy department at Box Hill Hospital so that he did not inadvertently become aware of who was attending physiotherapy. Also the third investigator (KB....) usually reminded the subjects not to tell CFW whether or not they had been to physiotherapy prior to review appointment..."

No blinding of number of physiotherapy treatment sessions

From email (27 July 2001): "Two subjects were excluded prior to data analysis. One subject who was randomly allocated to the non-physiotherapy group attended physiotherapy at a private practice, based on a referral by her general practitioner made one day after the initial measurements were taken. A second student [subject] randomly allocated to the physiotherapy group attended only his first appointment but failed to attend any subse- 
Watt 2000 (Continued)

\begin{tabular}{|c|c|c|}
\hline & & $\begin{array}{l}\text { quent appointment. Moreover this subject } \\
\text { had without consultation radically modi- } \\
\text { fied his plaster during the period of mobi- } \\
\text { lization" }\end{array}$ \\
\hline $\begin{array}{l}\text { Incomplete outcome data (attrition bias) } \\
\text { Longer term follow-up }\end{array}$ & Unclear risk & No longer-term follow-up \\
\hline Selective reporting (reporting bias) & Low risk & $\begin{array}{l}\text { No protocol available but the reported out- } \\
\text { comes appear to be consistent with the trial } \\
\text { investigators' intended outcomes }\end{array}$ \\
\hline Major baseline imbalance bias? & Low risk & No imbalance detected \\
\hline $\begin{array}{l}\text { Other performance bias (e.g. differential } \\
\text { expertise bias) }\end{array}$ & Unclear risk & $\begin{array}{l}\text { Comparability of other treatments, includ- } \\
\text { ing general practitioner referrals for ther- } \\
\text { apy in the control group, not confirmed for } \\
\text { this pragmatic trial }\end{array}$ \\
\hline
\end{tabular}

ADL: activities of daily living

AO: Arbeitsgemeinschaft fur Osteosynthesefragen / Association for the Study of Internal Fixation (or ASIF)

CRPS-1: complex regional pain syndrome type 1 (often referred to as reflex sympathetic dystrophy in older included studies)

DASH: Disabilities of the Arm, Shoulder and Hand outcome measure

DRUJ: distal radial ulnar joint

OT: occupational therapy

PEMF: pulsed electromagnetic field

POP: plaster of Paris

PRWE: Patient-Rated Wrist Evaluation

QOL: quality of life

ROM: range of movement

VAS: visual analogue scale or score

\section{Characteristics of excluded studies [ordered by study ID]}

\begin{tabular}{ll}
\hline Study & Reason for exclusion \\
\hline ACTRN12606000160538 & $\begin{array}{l}\text { This registered study (document submitted: 01/05/2006) aimed to compare 'aggressive' physiotherapy } \\
\text { versus 'regular' physiotherapy following fragment specific fixation of distal radius fractures in } 60 \text { adults. } \\
\text { The intended date for starting recruitment was 01/06/2015; but the status is listed as 'Not yet recruiting'. } \\
\text { It is very unlikely that this trial ever started. No response was received to queries on status sent to Dr } \\
\text { Goldbloom (email bounced) and then Dr Blackmore on 01/06/2014 }\end{array}$ \\
\hline Ayhan 2014 & $\begin{array}{l}\text { This randomised trial compared core stabilisation training and traditional arm rehabilitation versus } \\
\text { traditional arm rehabilitation alone for three days/week for six weeks in } 27 \text { patients with arm injuries, } 13 \\
\text { of whom had distal radius fractures. This study was excluded because of the more general rehabilitation }\end{array}$
\end{tabular}

Rehabilitation for distal radial fractures in adults (Review) 

just 4 patients with distal radius fracture and presenting results for one person in the mirror therapy group and one in the control group. This study is far too small to be included

Can 2001

Non-randomised comparative study: participants were matched according to their age, sex, pain intensity level, range of motion and treatment procedures before study completion

Coyle 1998

Trial of 8 participants involving a "single subject, multi-element design" comparison of two techniques of passive immobilisation: passive sustained stretches and oscillations, their order and timing within a series of 6 treatment sessions. The study design, basically resulting in comparisons involving individual participants, was considered potentially misleading and unsuitable for this review

Haren 2000

Randomised trial of manual lymph drainage in 29 participants treated with external fixation. Only oedema reported; no recording of functional outcomes

Haren 2004

Randomised trial of manual lymph drainage in 51 participants with oedema after fixation of their fracture. Only oedema was reported; no recording of functional outcomes

Hunt 2001

Non-randomised study. Prospective series of 13 participants compared with 13 retrospective control participants

Jarvis 2001

Non-randomised study involving a prospective series and retrospective control series

Kingston 2014

This randomised controlled trial, which was set up as a feasibility study, evaluated the effect on compliance with home exercise protocols of a DVD provided as well as brochures compared with brochures alone in 53 patients with traumatic hand injuries. Of these 23 had received internal fixation (TriMed wrist fixation) for distal radius fracture (11 with DVD versus 12 no DVD). There was no reporting of functional outcomes or other outcomes listed in Types of outcome measures

Lohstrater 2006

This was an inadequately reported interim analysis of a trial that aimed to recruit 200 patients with distal radius fractures. The trial compared special hand management versus standard treatment. Of the 136 participants that had been recruited "in the study to date", 111 had been followed up at 9 months. The report stated that "The study will continue till achieving the calculated sample size". Efforts (25/02/2013 and 06/03/2013) to contact the trialists were unsuccessful and no other trial report has been found. The study is excluded since this is an interim analysis, which fails to report the numbers randomised or followed-up in each group and only presents the data graphically

Naik 2007

Poorly written report of a trial comparing the Maitland versus Mulligan mobilisation technique in the management of post-surgical (external fixation) Colles' fracture. Request for email contact for first and second authors sent to their institution on 25/02/2013; with reply received 26/02/2013. "First author email not available at present with us, about other two coauthor email as mentioned Jeba Chitra - jebachitra@hotmail.com, Subash Khatri - kats003@india.com”. Request for information on study methods including method of randomisation, length of follow-up, details and timing of interventions, actual number of participants, details of outcome measures, sent to Prof Chitra on 28/02/2013. She responded $03 / 03 / 2013$, needing more time as she needed to look through the thesis materials. This was not forthcoming despite a reminder. This study is excluded as the trial report is insufficiently reported and further details have been unobtainable 
Nikolova 1969 Comparative study involving participants with established complications (reflex sympathetic dystrophy, delayed callus formation, painful joint stiffness), an unknown number of whom had had fractures of the distal radius. There is no indication that this is a randomised trial and the treatment of established complications is not in the scope of this review

Oskarsson 1997

Non-randomised prospective comparative study. Referral to physiotherapy based on patient request and/or severe stiffness

Pasila 1980

Randomised trial of Movelat cream versus placebo in 104 Colles' fracture participants with persistent problems with mobilisation of their wrist and hand after removal of plaster cast at five weeks. Drug trials are not included in this review

Ramesh 1998

Non-randomised study. Prior treatment of participants differed in the two groups

Rodrick 2004

Pilot study - thus probably small - reported in a conference abstract that compared retrograde massage versus manual oedema mobilisation in a mixed population with wrist disorders. There was no mention of distal radial fractures

Schwartz-Jensen 2002

We were unable to locate a source to contact for the information required for the inclusion of this pilot study, reported only in a conference abstract, testing individual occupational therapy during the immobilisation period in 29 people with a distal radial fracture

Wang 2012

Quasi-randomised trial, reported in Chinese with English abstract, comparing ultrashort wave and magnetic therapy versus "western medicine" (intravenous beta-aescin injection) versus control in 90 patients after "manual reduction and small splint external fixation". Only reduction of swelling was reported; no reporting of functional outcomes

Woodbridge 2003

This randomised trial, which started mid-1998, included 80 participants, aged 18 and above, with distal radial fractures referred for rehabilitation after plaster cast removal at Derbyshire Royal Infirmary, Derby, UK. It tested the order of provision of rehabilitation which comprised 4 sessions a week (2 physiotherapy and 2 occupational therapy): physiotherapy session before occupational therapy session versus occupational therapy session before physiotherapy session. The length of follow-up was six months and outcomes collected were: range of motion, grip strength, pinch strength, time to meet discharge criteria (attainment of $1 / 2$ grip strength and $2 / 3$ active ROM), Jebsen test (dexterity), DASH Repeated contact was made with the lead investigator, Sarah Woodbridge, who confirmed that the trial had been completed. On last contact (9 November 2005), Sarah Woodbridge revealed that preparation of the written report had been delayed due to unforeseen circumstances. However, some consideration would be given to the request for a copy of the report (if it could be found) sent to the original funders of the trial (Action Research). No report was forthcoming from the lead investigator and this trial remains unpublished and it seems unlikely it will be published. Hence, our decision to exclude this trial mineral content. No reporting of functional outcome 


\section{Characteristics of studies awaiting assessment [ordered by study ID]}

\section{Duvoric 2005}

\begin{tabular}{ll}
\hline Methods & “patients were divided into 3 equal group" \\
\hline Participants & 30 patients with distal radius fracture \\
\hline Interventions & $\begin{array}{l}\text { Worn during rehabilitation programme } \\
\text { 1. Custom-fit circumferential static wrist orthosis } \\
\text { 2. Off the shelf circumferential static wrist orthosis } \\
\text { 3. No orthosis }\end{array}$ \\
\hline Outcomes & $\begin{array}{l}\text { Pain, oedema, range of the wrist motion, quality and strength of pinch and hand grasp, patient's assessment of the } \\
\text { effects of rehabilitation }\end{array}$ \\
\hline Notes & \begin{tabular}{l} 
We have been unable to obtain a copy of this report to determine methods or appraise the nature of the intervention \\
\hline
\end{tabular}
\end{tabular}

McPhate 1998

\begin{tabular}{|c|c|}
\hline Methods & Randomised controlled trial \\
\hline Participants & 32 women over 50 who had sustained a Colles fracture \\
\hline Interventions & $\begin{array}{l}\text { All participants received a comprehensive regimen of home exercises which were progressed at each session } \\
\text { 1. Passive mobilisation of wrist and carpal bones for extension and supination, plus exercise instruction } \\
\text { 2. Exercise instruction only }\end{array}$ \\
\hline Outcomes & Pain (VAS), active wrist extension (goniometer), grip strength (Jamar dynamometer) \\
\hline Notes & $\begin{array}{l}\text { Confirmation received via Sandra Kay (17 January 2003) that the study was in the process of being written up by } \\
\text { Margaret McPhate (c/o Physiotherapy Department, St Vincent's Public Hospital, } 41 \text { Victoria Parade, Fitzroy, Victoria } \\
\text { 3065, Australia) } \\
\text { Email requesting direct confirmation and further details sent to Margaret McPhate on } 22 \text { January 2003. Requests for } \\
\text { clarification on current status sent } 6 \text { and } 19 \text { February 2004. Correspondence from Sandra Kay (27 October 2005) } \\
\text { notified that Margaret McPhate was now in Canberra. The study was pending some reanalysis of the data. Email } \\
\text { from Kim Brock at St Vincent's on } 5 \text { December } 2005 \text { confirmed that the study was not yet published }\end{array}$ \\
\hline
\end{tabular}

\section{NCT00816998}

Methods Randomised parallel assignment open label trial

Participants 39 participants, aged 18 to 85 years, with closed unstable distal radius fracture treated with plate and screw fixation followed by a plaster splint

Interventions 1. Early physical therapy (including active range of motion) started approximately one week following surgery

2. Delayed physical therapy started six weeks following surgery 


\section{NCT00816998 (Continued)}

Outcomes Follow-up: 12 months (also 6 months)

Primary outcome: range of motion

Secondary outcomes: pinch strength, grip strength, pain, return to work, DASH \& PRWE questionnaires

Notes Trial retrospectively registered: January 5, 2009

Started October 2006; ended February 2010 (final data collection date for primary outcome measure)

Last updated: April 6, 2011 (as completed)

No publication of this trial (conducted at the Mayo clinic, Rochester, Minnesota, US) identified

\section{NCT01262807}

Methods Randomised single blind trial

Participants Planned enrolment: 70 participants, aged 18 or above, with distal radius fracture treated non-operatively

Interventions All participants receive cast immobilisation for 6 weeks

1. A specific set of standardised exercises will be taught to patients who are randomised to the intervention group.

They will be instructed to do these exercises daily while in-cast. They will have an exercise log to track adherence

2. Standard care

Outcomes Follow-up: 1 year (also 6 weeks and 6 months)

Primary outcome: change in range of motion

Secondary outcomes: Complications (6 weeks)

Notes Trial registered: December 15, 2010

Started December 2010; estimated end date December 2012 (final data collection date for primary outcome measure)

Last updated: December 2010 (recruiting); current status is unknown

No publication found of this trial (conducted at the University of Manitoba, Winnipeg, Manitoba, Canada)

Lead contact: Jamie Dubberley jdubberley@panamclinic.com

\section{NCT01589627}

\begin{tabular}{ll} 
Methods & Randomised parallel assignment open label trial \\
\hline Participants & $\begin{array}{l}\text { Planned enrolment: } 50 \text { patients, aged } 18 \text { to } 80 \text { years, with surgically-treated distal radius fracture that have wrist } \\
\text { flexion contracture upon follow-up }\end{array}$ \\
\hline Interventions & $\begin{array}{l}\text { 1. Wrist Extension Dynasplint plus standard of care (physical therapy and NSAIDs) } \\
\text { 2. Standard of care (physical therapy and NSAIDs) }\end{array}$ \\
\hline Outcomes & $\begin{array}{l}\text { Follow-up: } 12 \text { weeks } \\
\text { Primary (and only listed) outcome: change in maximal active range of motion in wrist extension }\end{array}$ \\
\hline Notes & $\begin{array}{l}\text { Sponsor: Dynasplint Systems, Inc. } \\
\text { Trial registered: April 27, 2012 } \\
\text { Started: December 2010; } \\
\text { Estimated end date December } 2016 \text { (final data collection date for primary outcome measure) }\end{array}$
\end{tabular}




\section{NCT01589627 (Continued)}

Last updated: April 2012: "This study is not yet open for participant recruitment"

Contact (no email provided or found): Stacey Berner, Advanced Centers for Orthopaedic Surgery and Sports Medicine, 10 Crossroads \#210 Owings Mills, MD 21117, USA

Linked cohort study (also not started as of July 2013) that was meant to end at the same time: https://clinicaltrials. gov/show/NCT01032356

Poorly edited trial registration document: intervention describes knee extension Dynasplint

\section{Oken 2011}

\begin{tabular}{ll}
\hline Methods & Randomised (via "drawing of lots") \\
\hline Participants & $\begin{array}{l}57 \text { participants with non-displaced, stable distal radius fractures treated with short cast immobilisation after closed } \\
\text { reduction }\end{array}$ \\
\hline Interventions & $\begin{array}{l}\text { 1. Hospital based therapy: passive range of motion (ROM) and gentle stretching exercises of the wrist supervised by } \\
\text { a hand specialist at the hand rehabilitation department } \\
\text { 2. Home based therapy: ROM and stretching exercises performed at home by participants themselves }\end{array}$ \\
\hline Outcomes & $\begin{array}{l}\text { Follow-up: up to end of treatment } \\
\text { Wrist ROM, hand oedema, and grip/pinch strength }\end{array}$ \\
\hline Notes & $\begin{array}{l}\text { Queries on methods sent to Dr Oken on 01/06/2014. This included a request for an explanation of the imbalance } \\
\text { in recruitment in the two groups (37 versus 20) and clarification on timing of randomisation and therapy and on } \\
\text { the exclusion of patients with complex regional pain syndrome }\end{array}$ \\
\hline
\end{tabular}

Schmidt 2013

\begin{tabular}{ll}
\hline Methods & Randomised trial \\
\hline Participants & 57 participants with surgically treated distal radius fracture \\
\hline Interventions & $\begin{array}{l}\text { 1. Compression glove } \\
\text { 2. Control (elastic 'wicklung') }\end{array}$ \\
\hline Outcomes & $\begin{array}{l}\text { Length of follow-up: } 42 \text { days (also: } 2,4,6,10,14,21 \text { and } 32 \text { days) } \\
\text { Swelling } \\
\text { Range of motion }\end{array}$ \\
\hline Notes & $\begin{array}{l}\text { Translation required: trial is published in German with an English abstract } \\
\text { However, it is clear that the functional data are very limited being restricted to overall range of motion data. Thus it } \\
\text { is not certain that this trial warrants inclusion }\end{array}$ \\
\hline
\end{tabular}


Characteristics of ongoing studies [ordered by study ID]

\section{ACTRN12612000118808}

\begin{tabular}{|c|c|}
\hline Trial name or title & Does exercise following distal radius fracture improve activity? A multi-centre randomised controlled trial \\
\hline Methods & Randomised controlled trial \\
\hline Participants & 30 patients, aged 21 years or over, who were managed in a cast \\
\hline Interventions & $\begin{array}{l}\text { 1. Professional advice plus a progressive exercise programme in three standard consultations (approximately } \\
20 \text { to } 30 \text { minutes) held in weeks } 7,9 \text { and } 11 \text { (from time of injury) } \\
\text { 2. Professional advice plus three standard physiotherapy consultations in weeks } 7,9 \text { and } 11 \text { which will last } \\
\text { between } 20 \text { to } 30 \text { minutes }\end{array}$ \\
\hline Outcomes & $\begin{array}{l}\text { Follow-up: } 24 \text { weeks (also } 5 \text { to } 6 \text { weeks) } \\
\text { Primary outcomes: activity limitations assessed using activity-specific section of the PRWE Questionnaire; } \\
\text { quick-DASH; arm usage assessed using accelerometers } \\
\text { Secondary outcomes: wrist flexion, extension and supination range of motion, grip strength, pain assessed } \\
\text { using pain-specific section of the PRWE Questionnaire, adherence to home exercises }\end{array}$ \\
\hline Starting date & $\begin{array}{l}\text { Start date: first enrolment was } 26 / 07 / 2012 \\
\text { Recruitment status: completed, last enrolment was } 11 / 06 / 2013\end{array}$ \\
\hline Contact information & $\begin{array}{l}\text { Ms Andrea Bruder, Department of Physiotherapy, La Trobe University, Bundoora, Victoria 3086, Australia } \\
\text { Email: a.bruder@latrobe.edu.au }\end{array}$ \\
\hline Notes & $\begin{array}{l}\text { Prospectively registered } 24 \text { January } 2012 \\
\text { No mention of publication on contact author's website (checked 03/03/2015) }\end{array}$ \\
\hline
\end{tabular}

JPRN-UMIN000015003

Trial name or title Effectiveness of occupational therapy after volar locking plate fixation of the distal radius fracture. A prospective randomised controlled trial

\begin{tabular}{ll}
\hline Methods & Randomised controlled trial \\
\hline Participants & $\begin{array}{l}\text { Planned: } 56 \text { patients, aged } 20 \text { years or over, who have had open reduction and volar locking plate fixation of } \\
\text { the distal radius fracture }\end{array}$ \\
\hline Interventions & $\begin{array}{l}\text { 1. Occupational therapy (2 times/week, during the ten weeks after surgery) and home exercise instruction by } \\
\text { the attending doctor at every visit } \\
\text { 2. Home exercise instruction by the attending doctor at every visit }\end{array}$ \\
\hline Outcomes & $\begin{array}{l}\text { Follow-up: no information } \\
\text { Primary outcome: range of motion of the wrist and the forearm } \\
\text { Secondary outcomes: grip strength, pinch strength, Quick DASH, PRWE, pain evaluated by Visual Analogue } \\
\text { Scale (VAS) }\end{array}$ \\
\hline Starting date & $\begin{array}{l}\text { 01/09/2014 (anticipated; see Notes) } \\
\text { Estimated completion date: no information }\end{array}$
\end{tabular}

Rehabilitation for distal radial fractures in adults (Review) 


\begin{tabular}{ll}
\hline Contact information & Kazushige Gamo \\
& Bell Land General Hospital \\
& Orthopaedic Surgery \\
& 500-3 Higashiyama, Naka-ku, Sakai-shi \\
& Osaka 599-8247, Japan \\
& kaz-gamo@umin.ac.jp \\
\hline Notes & Date of registration: 31 August 2014 \\
& Recruitment status: not yet recruiting (on 16 September 2014) \\
& Claim: "single blind: participants are blinded" \\
\hline
\end{tabular}

\section{NCT01118715}

\begin{tabular}{ll} 
Trial name or title & Use of compression glove to prevent complications after distal radius fractures: a randomised controlled trial \\
\hline Methods & Randomised parallel assignment open label trial \\
\hline
\end{tabular}

Participants $\quad$ Planned enrolment: 460 patients, aged between 18 and 85, with unstable unilateral distal radius fractures requiring surgical stabilisation

\begin{tabular}{|c|c|}
\hline Interventions & $\begin{array}{l}\text { 1. Compression glove. This is incorporated into the patient's splint for } 2 \text { weeks post-op, and worn underneath } \\
\text { the patient's cast for } 3 \text { weeks. The patient then wears the glove at night after cast removal } \\
\text { 2. 'Standard recovery procedures': splint worn for } 2 \text { weeks post-op, followed by a short arm cast worn for the } \\
\text { next } 3 \text { weeks }\end{array}$ \\
\hline Outcomes & $\begin{array}{l}\text { Follow-up: } 6 \text { months (also } 2 \& 5 \text { weeks and } 3 \text { months) } \\
\text { Primary outcome: carpal tunnel syndrome } \\
\text { Secondary outcomes: complex regional pain syndrome, oedema, grip strength, DASH, pain, time to recovery }\end{array}$ \\
\hline Starting date & $\begin{array}{l}\text { April } 2010 \\
\text { Estimated completion date: April } 2014 \text { (Final data collection date for primary outcome measure) }\end{array}$ \\
\hline Contact information & $\begin{array}{l}\text { Michael S Shuler (J\&M Shuler) } \\
\text { Athens Orthopedic Clinic } \\
\text { Athens, Georgia } \\
\text { United States, } 30606 \\
\text { msimmss@hotmail.com }\end{array}$ \\
\hline Notes & $\begin{array}{l}\text { Date of registration: April 30, 2010; last updated: January 28, } 2013 \\
\text { Recruitment status: recruiting (January 2013) } \\
\text { The contact author is part of a medical company: J\&M Shuler } \\
\text { Abstract reporting results for } 48 \text { participants available (Harris 2011). Response to query on trial status received } \\
\text { from Michael Shuler on } 01 / 06 / 2015 \text { stated: "We plan to publish this paper in the fairly near future. The } \\
\text { results showed significant decreased edema with the glove and decreased post-op pain in the initial periods. } \\
\text { No long-term benefits as would be expected. The sample size over } 100 \text { subjects was not enough to show } \\
\text { statistical significance regarding post-op CTS and RSD [reflex sympathetic dystrophy]/CRPS" }\end{array}$ \\
\hline
\end{tabular}




\begin{tabular}{ll} 
Trial name or title & $\begin{array}{l}\text { Effects of motor cognitive training on functional loss through immobilization after osteoporotic distal radius } \\
\text { fractures: a randomised clinical pilot study in elderly patients }\end{array}$ \\
\hline Methods & Randomised controlled trial with blinded assessor \\
\hline Participants & Included 27 women aged 60 years and older having a distal radius fracture and sufficient cognitive function \\
\hline Interventions & $\begin{array}{l}\text { All groups visited at home for therapy sessions } 5 \text { times per week for the first } 3 \text { weeks and } 3 \text { times per week } \\
\text { for weeks } 4 \text { to } 6\end{array}$ \\
$\begin{array}{l}\text { 1. Participants imagine movements of the fractured upper extremity without executing them (mental practice) } \\
\text { 2. Participants receive a mirror therapy programme consisting of the performance } \\
\text { of functional movement synergies using the unaffected forearm, wrist, and hand } \\
\text { 3. Control: participants complete a relaxation training regimen } \\
\text { All participants receive usual care by the general practitioner }\end{array}$
\end{tabular}

\begin{tabular}{ll} 
Outcomes & $\begin{array}{l}\text { Follow-up: } 12 \text { weeks (also } 3 \text { and } 6 \text { weeks) } \\
\text { Primary outcome: PRWE } \\
\text { Secondary outcome: DASH, objective impairment (range of motion, grip force; quality of life (EuroQol-5D) }\end{array}$ \\
\hline Starting date & $\begin{array}{l}\text { August } 2010 \\
\text { Completion date: October } 2014 \text { (Final data collection date for primary outcome measure) }\end{array}$ \\
\hline Contact information & $\begin{array}{l}\text { Professor Nadja Schott } \\
\text { Department of Sports and Exercise Science, University of Stuttgart, Allmandring 28, 70569 Stuttgart, Ger- } \\
\text { many } \\
\text { nadja.schott@inspo.uni-stuttgart.de }\end{array}$ \\
\hline
\end{tabular}

\section{NCT01518179}

Trial name or title The effect of wearing made-to-measure compression gloves on rehabilitation following DRF

\begin{tabular}{ll}
\hline Methods & Randomised parallel assignment open label trial \\
\hline Participants & $\begin{array}{l}\text { Planned: } 120 \text { participants, aged } 18 \text { to } 85 \text { years, referred to rehabilitation } 4 \text { to } 6 \text { weeks after surgical or } \\
\text { conservative treatment for distal radius fracture, with or without involvement of the ulna, who have at least } \\
\text { two of the following: pain; limited range of motion of the fingers and the wrist; limited strength (weakness of } \\
\text { the hand/fingers); swelling/oedema; limited hand functions during performance of activities of daily living } \\
\text { (ADL) }\end{array}$
\end{tabular}

$\begin{array}{ll}\text { Interventions } & \text { Routine treatment and follow-up } \\ \text { 1. With made-to-measure compression gloves (received within a week of enrolment) } \\ \text { 2. Without compression glove }\end{array}$

Outcomes Follow-up: assessment by an occupational therapist at 2, 4, and 8 week after enrolment; telephone interview at 3, 6 and 12 months Primary outcome: PRWE at 1 year 


\section{NCT01518179 (Continued)}

Secondary outcomes (at 10 weeks): swelling of the hand and fingers; range of motion of the fingers and wrist; strength of the hand; pain (using VAS as part of PRWE); overall satisfaction of using the compression gloves

\begin{tabular}{ll} 
Starting date & $\begin{array}{l}\text { April } 2012 \\
\text { Estimated completion date: Dece }\end{array}$ \\
\hline Contact information & $\begin{array}{l}\text { Dr Uzi Milman } \\
\text { Director, Clinical Research Unit } \\
\text { Clalit Health Services }\end{array}$ \\
& $\begin{array}{l}\text { Haifa } \\
\text { Israel } \\
\text { uzimy@netvision.net.il }\end{array}$ \\
\hline
\end{tabular}

Notes Date of registration: January 21, 2012

Confirmed as recruiting participants: May 2012

\section{NCT01693094}

Trial name or title A randomised trial measuring the effect of decision aids on patients' satisfaction, conflict of decision-making and clinical outcome

Methods Randomised parallel assignment open label trial

Participants Planned enrolment: 126 patients, aged 18 and above, with diagnosis of moderate or severe:

a. Trapeziometacarpal arthrosis

b. Carpal tunnel syndrome

c. Cubital tunnel syndrome

d. Distal radius fractures

e. Trigger finger

Interventions

1. Decision aid. Patients provided with a decision aid, which they can complete in a separate room and take home. The decision aids include information on the disease/condition, treatment options, benefits, risks, scientific uncertainties, and probabilities of potential outcomes tailored to the patient's health risks factors. Additionally, it includes values clarifications such as describing outcomes in functional terms, asking patients to consider which benefits and risks matter most to them, and guidance in the steps of decision making and discussing their decision with family/friends. It is interactive and dynamic, helping patients clarify their preferences and come to a decision that feels best to them

2. No decision aid. Patients given American Society of Surgery of the Hand brochure as standard treatment

Outcomes

Follow-up: 6 months (also 6 weeks)

Primary outcome: Decision conflict scale

Secondary outcomes: 11-point ordinal satisfaction scale; knowledge questionnaire (diagnosis and treatment); stage of decision making; decision self efficacy scale; acceptability; pain self efficacy questionnaire; Disabilities of the Arm, Shoulder and Hand Quick Questionnaire (Quick-DASH); EuroQol-5D-5L; decision regret scale

Starting date

September 2012

Estimated primary completion date: December 2015 (final data collection date for primary outcome measure) 


\section{NCT01693094 (Continued)}

\begin{tabular}{ll}
\hline Contact information & Prof David Ring \\
& Director of Research \\
& Hand Service \\
& Massachusetts General Hospital \\
& Boston \\
& Massachusetts \\
& USA \\
& dring@partners.org \\
\hline Notes & Mixed population and thus separate data for distal radial fractures would need to be sought \\
& $\begin{array}{l}\text { Date of registration: September 13, 2012 } \\
\text { Status August 5, 2014: "study is enrolling participants by invitation only" }\end{array}$ \\
\hline
\end{tabular}

\section{NCT01921062}

Trial name or title Kinesthetic motor imagery training during immobilisation to improve wrist functional outcome after a distal radius fracture in women of $45-75$ years of age

\begin{tabular}{ll}
\hline Methods & Randomised parallel assignment open label trial \\
\hline Participants & Planned: 52 women, aged 45 to 75 years, treated with a cast for a non-comminuted distal radius fracture \\
\hline Interventions & $\begin{array}{l}\text { 1. Motor imagery: patients allocated to this arm perform kinaesthetic motor imagery during the immobilisa- } \\
\text { tion period }\end{array}$ \\
\begin{tabular}{l} 
2. Control group: standard treatment only \\
\hline
\end{tabular}
\end{tabular}

Outcomes

Follow-up: 2 weeks, and immediately after and 2 days after cast removal

Primary outcome: hand function at 2 days after cast removal using the Patient-Rated Wrist Hand Evaluation (PRWHE)

Secondary outcomes: grip strength (power grip strength, key-pinch grip strength, three-jaw pinch strength, two-point pinch strength); dexterity using the Sequential Occupational Dexterity Assessment (SODA), pain using a Visual Analogue Scale (VAS), range of motion; hand function (Patient-Rated Wrist Hand Evaluation: PRWHE) at 2 weeks

Starting date July 2011

Estimated completion date: July 2015 (final data collection date for primary outcome measure)

Contact information Dieuwke C Broekstra

University Medical Centre Groningen

Groningen

Netherlands, 9700B

d.c.broekstra@umcg.nl 
Trial name or title

\begin{tabular}{|c|c|}
\hline Methods & Randomised parallel assignment open label trial \\
\hline Participants & $\begin{array}{l}\text { Planned: } 120 \text { patients, aged } 18 \text { to } 70 \text { years, treated with volar locked plate fixation of an extra-articular distal } \\
\text { radius fracture }\end{array}$ \\
\hline Interventions & $\begin{array}{l}\text { Post-surgery: } \\
\text { 1. Early mobilisation (cast to } 2 \text { to } 3 \text { days), weightbearing and physical therapy } \\
\text { 2. Late mobilisation (cast for two weeks), non-weightbearing, and instructions for home exercises alone }\end{array}$ \\
\hline Outcomes & $\begin{array}{l}\text { Follow-up: } 2 \text { years } \\
\text { Primary outcomes: Short version of "Disabilities of the Arm, Shoulder and Hand" (Quick-DASH), Short } \\
\text { Form } 36 \text { (SF-36) } \\
\text { Secondary outcomes: Patient Rated Wrist Evaluation (PRWE), Euro-Qol } 5 \text { dimension score (EQ-5d), pain } \\
\text { scores on the Visual Analog Scale (VAS), radiological findings (X-ray), range of motion (ROM), cost analysis } \\
\text { (cost of treatment, sick-leave, complications and other socio-economical parameters) }\end{array}$ \\
\hline Starting date & $\begin{array}{l}\text { January } 2012 \\
\text { Estimated completion date: February } 2016 \text { (final data collection date for primary outcome measure) }\end{array}$ \\
\hline Contact information & $\begin{array}{l}\text { Professor Jan Erik Madsen } \\
\text { University Hospital, Akershus } \\
\text { Oslo, Lorenskog } \\
\text { Norway }\end{array}$ \\
\hline Notes & $\begin{array}{l}\text { Date of registration: December 10, } 2013 \\
\text { Confirmed as ongoing but not recruiting participants: February } 2015\end{array}$ \\
\hline
\end{tabular}

DASH: Disability of the Arm, Shoulder, and Hand questionnaire

PRWE: Patient-Rated Wrist Evaluation

PRWHE: Patient-Rated Wrist Hand Evaluation

ROM: range of movement

VAS: visual analogue scale 
DATA ANDANALYSES

Comparison 1. Early (during immobilisation) occupational or hand therapy versus no intervention (control)

\begin{tabular}{|c|c|c|c|c|}
\hline Outcome or subgroup title & $\begin{array}{l}\text { No. of } \\
\text { studies }\end{array}$ & $\begin{array}{c}\text { No. of } \\
\text { participants }\end{array}$ & Statistical method & Effect size \\
\hline $\begin{array}{l}1 \text { Meeting criteria for attendance } \\
\text { of post-immobilisation hand } \\
\text { therapy group }\end{array}$ & 1 & & Risk Ratio (M-H, Fixed, 95\% CI) & Totals not selected \\
\hline $\begin{array}{l}2 \text { Grip strength }(\mathrm{kg}) \text { at } 4 \text { weeks } \\
\text { (post-immobilisation) }\end{array}$ & 1 & & Mean Difference (IV, Fixed, 95\% CI) & Totals not selected \\
\hline $\begin{array}{l}3 \text { Range of motion at } 4 \text { weeks } \\
\text { (post-immobilisation) }\end{array}$ & 1 & & Mean Difference (IV, Fixed, 95\% CI) & Totals not selected \\
\hline 3.1 Pronation (degrees) & 1 & & Mean Difference (IV, Fixed, 95\% CI) & $0.0[0.0,0.0]$ \\
\hline 3.2 Supination (degrees) & 1 & & Mean Difference (IV, Fixed, 95\% CI) & $0.0[0.0,0.0]$ \\
\hline 3.3 Flexion (degrees) & 1 & & Mean Difference (IV, Fixed, 95\% CI) & $0.0[0.0,0.0]$ \\
\hline 3.4 Extension (degrees) & 1 & & Mean Difference (IV, Fixed, 95\% CI) & $0.0[0.0,0.0]$ \\
\hline 3.5 Radial deviation (degrees) & 1 & & Mean Difference (IV, Fixed, 95\% CI) & $0.0[0.0,0.0]$ \\
\hline 3.6 Ulnar deviation (degrees) & 1 & & Mean Difference (IV, Fixed, 95\% CI) & $0.0[0.0,0.0]$ \\
\hline $\begin{array}{l}4 \text { Oedema }(\mathrm{mL}) \text { at } 4 \text { weeks } \\
\text { (post-immobilisation) }\end{array}$ & 1 & & Mean Difference (IV, Fixed, 95\% CI) & Totals not selected \\
\hline $\begin{array}{l}5 \text { Any pain at rest at } 4 \text { weeks } \\
\text { (post-immobilisation) }\end{array}$ & 1 & & Risk Ratio (M-H, Fixed, 95\% CI) & Totals not selected \\
\hline $\begin{array}{l}6 \text { Finger mobility at } 4 \text { weeks } \\
\text { (post-immobilisation) }\end{array}$ & 1 & & Mean Difference (IV, Fixed, 95\% CI) & Totals not selected \\
\hline $\begin{array}{l}\text { 6.1 Kapandji score (thumb } \\
\text { opposition, } 1 \text { to } 10 \text { locations of } \\
\text { increasing difficulty) }\end{array}$ & 1 & & Mean Difference (IV, Fixed, 95\% CI) & $0.0[0.0,0.0]$ \\
\hline $\begin{array}{l}6.2 \text { Index finger TAM (total } \\
\text { active motion) (degrees) }\end{array}$ & 1 & & Mean Difference (IV, Fixed, 95\% CI) & $0.0[0.0,0.0]$ \\
\hline $\begin{array}{l}6.3 \text { Middle finger TAM } \\
\text { (degrees) }\end{array}$ & 1 & & Mean Difference (IV, Fixed, 95\% CI) & $0.0[0.0,0.0]$ \\
\hline 6.4 Ring finger TAM (degrees) & 1 & & Mean Difference (IV, Fixed, 95\% CI) & $0.0[0.0,0.0]$ \\
\hline $\begin{array}{l}\text { 6.5 Little finger TAM } \\
\text { (degrees) }\end{array}$ & 1 & & Mean Difference (IV, Fixed, 95\% CI) & $0.0[0.0,0.0]$ \\
\hline 7 Complications & 2 & & Risk Ratio (M-H, Fixed, 95\% CI) & Totals not selected \\
\hline 7.1 CRPS-1 & 2 & & Risk Ratio (M-H, Fixed, 95\% CI) & $0.0[0.0,0.0]$ \\
\hline 7.2 Median nerve compression & 1 & & Risk Ratio (M-H, Fixed, 95\% CI) & $0.0[0.0,0.0]$ \\
\hline 7.3 Ulnar nerve compression & 1 & & Risk Ratio (M-H, Fixed, 95\% CI) & $0.0[0.0,0.0]$ \\
\hline 7.4 Tendon rupture & 1 & & Risk Ratio (M-H, Fixed, 95\% CI) & $0.0[0.0,0.0]$ \\
\hline
\end{tabular}




\begin{tabular}{|c|c|c|c|c|}
\hline Outcome or subgroup title & $\begin{array}{l}\text { No. of } \\
\text { studies }\end{array}$ & $\begin{array}{c}\text { No. of } \\
\text { participants }\end{array}$ & Statistical method & Effect size \\
\hline 1 Grip strength (kg) & 1 & & Mean Difference (IV, Fixed, 95\% CI) & Totals not selected \\
\hline 1.16 weeks & 1 & & Mean Difference (IV, Fixed, 95\% CI) & $0.0[0.0,0.0]$ \\
\hline 1.210 weeks & 1 & & Mean Difference (IV, Fixed, 95\% CI) & $0.0[0.0,0.0]$ \\
\hline 2 Pinch strength $(\mathrm{kg})$ & 1 & & Mean Difference (IV, Fixed, 95\% CI) & Totals not selected \\
\hline 2.16 weeks & 1 & & Mean Difference (IV, Fixed, 95\% CI) & $0.0[0.0,0.0]$ \\
\hline 2.210 weeks & 1 & & Mean Difference (IV, Fixed, 95\% CI) & $0.0[0.0,0.0]$ \\
\hline 3 Range of motion (degrees) & 1 & & Mean Difference (IV, Fixed, 95\% CI) & Totals not selected \\
\hline $\begin{array}{l}\text { 3.1 Flexion/extension at } 6 \\
\text { weeks }\end{array}$ & 1 & & Mean Difference (IV, Fixed, 95\% CI) & $0.0[0.0,0.0]$ \\
\hline $\begin{array}{l}3.2 \text { Flexion/extension at } 10 \\
\text { weeks }\end{array}$ & 1 & & Mean Difference (IV, Fixed, 95\% CI) & $0.0[0.0,0.0]$ \\
\hline $\begin{array}{l}3.3 \text { Supination/pronation at } 6 \\
\text { weeks }\end{array}$ & 1 & & Mean Difference (IV, Fixed, 95\% CI) & $0.0[0.0,0.0]$ \\
\hline $\begin{array}{l}3.4 \text { Supination/pronation at } \\
10 \text { weeks }\end{array}$ & 1 & & Mean Difference (IV, Fixed, 95\% CI) & $0.0[0.0,0.0]$ \\
\hline
\end{tabular}

Comparison 3. Early (during external fixation) digit mobilisation programme versus no intervention (control)

\begin{tabular}{|c|c|c|c|c|}
\hline Outcome or subgroup title & $\begin{array}{l}\text { No. of } \\
\text { studies }\end{array}$ & $\begin{array}{c}\text { No. of } \\
\text { participants }\end{array}$ & Statistical method & Effect size \\
\hline $\begin{array}{l}1 \text { Manual Ability Measure-36 - } \\
\text { Taiwan version } 45 \text { questions } \\
\text { (36 to 180: best result) }\end{array}$ & 1 & & Mean Difference (IV, Fixed, 95\% CI) & Totals not selected \\
\hline 1.1 At 3 weeks & 1 & & Mean Difference (IV, Fixed, 95\% CI) & $0.0[0.0,0.0]$ \\
\hline 1.2 At 7 weeks & 1 & & Mean Difference (IV, Fixed, 95\% CI) & $0.0[0.0,0.0]$ \\
\hline 1.3 At 12 weeks & 1 & & Mean Difference (IV, Fixed, 95\% CI) & $0.0[0.0,0.0]$ \\
\hline $\begin{array}{l}2 \text { Grip, pinch and 'three jaw } \\
\text { chuck' pinch strengths ( } \% \text { of } \\
\text { uninvolved hand) }\end{array}$ & 1 & & Mean Difference (IV, Fixed, 95\% CI) & Totals not selected \\
\hline 2.1 Grip power at 7 weeks & 1 & & Mean Difference (IV, Fixed, 95\% CI) & $0.0[0.0,0.0]$ \\
\hline 2.2 Grip power at 12 weeks & 1 & & Mean Difference (IV, Fixed, 95\% CI) & $0.0[0.0,0.0]$ \\
\hline 2.3 Pinch strength at 7 weeks & 1 & & Mean Difference (IV, Fixed, 95\% CI) & $0.0[0.0,0.0]$ \\
\hline 2.4 Pinch strength at 12 weeks & 1 & & Mean Difference (IV, Fixed, 95\% CI) & $0.0[0.0,0.0]$ \\
\hline $\begin{array}{l}2.5 \text { Three jaw chuck pinch } \\
\text { strength at } 7 \text { weeks }\end{array}$ & 1 & & Mean Difference (IV, Fixed, 95\% CI) & $0.0[0.0,0.0]$ \\
\hline $\begin{array}{l}\text { 2.6 Three jaw chuck pinch } \\
\text { strength at } 12 \text { weeks }\end{array}$ & 1 & & Mean Difference (IV, Fixed, 95\% CI) & $0.0[0.0,0.0]$ \\
\hline $\begin{array}{l}3 \text { Range of motion (\% of other } \\
\text { hand) }\end{array}$ & 1 & & Mean Difference (IV, Fixed, 95\% CI) & Totals not selected \\
\hline $\begin{array}{l}3.1 \text { 'Finger workspace' at } 12 \\
\text { weeks }\end{array}$ & 1 & & Mean Difference (IV, Fixed, 95\% CI) & $0.0[0.0,0.0]$ \\
\hline
\end{tabular}

Rehabilitation for distal radial fractures in adults (Review)

Copyright @ 2015 The Cochrane Collaboration. Published by John Wiley \& Sons, Ltd. 


\begin{tabular}{|c|c|c|c|c|}
\hline Outcome or subgroup title & $\begin{array}{l}\text { No. of } \\
\text { studies }\end{array}$ & $\begin{array}{c}\text { No. of } \\
\text { participants }\end{array}$ & Statistical method & Effect size \\
\hline $\begin{array}{l}1 \text { PRWE scores at } 2 \text { to } 3 \text { days after } \\
\text { cast removal }\end{array}$ & 1 & & Mean Difference (IV, Fixed, 95\% CI) & Totals not selected \\
\hline $\begin{array}{l}1.1 \text { Pain score ( } 0 \text { to } 50 \text { : worst } \\
\text { pain) }\end{array}$ & 1 & & Mean Difference (IV, Fixed, 95\% CI) & $0.0[0.0,0.0]$ \\
\hline $\begin{array}{l}1.2 \text { Activity/function score ( } 0 \\
\text { to } 50 \text { : worst function) }\end{array}$ & 1 & & Mean Difference (IV, Fixed, 95\% CI) & $0.0[0.0,0.0]$ \\
\hline $\begin{array}{l}2 \text { Range of motion at day } 2 \text { to } 3 \\
\text { after cast removal }\end{array}$ & 1 & & Mean Difference (IV, Fixed, 95\% CI) & Totals not selected \\
\hline 2.1 Pronation (degrees) & 1 & & Mean Difference (IV, Fixed, 95\% CI) & $0.0[0.0,0.0]$ \\
\hline 2.2 Supination (degrees) & 1 & & Mean Difference (IV, Fixed, 95\% CI) & $0.0[0.0,0.0]$ \\
\hline 2.3 Flexion (degrees) & 1 & & Mean Difference (IV, Fixed, 95\% CI) & $0.0[0.0,0.0]$ \\
\hline 2.4 Extension (degrees) & 1 & & Mean Difference (IV, Fixed, 95\% CI) & $0.0[0.0,0.0]$ \\
\hline 2.5 Radial deviation (degrees) & 1 & & Mean Difference (IV, Fixed, 95\% CI) & $0.0[0.0,0.0]$ \\
\hline 2.6 Ulnar deviation (degrees) & 1 & & Mean Difference (IV, Fixed, 95\% CI) & $0.0[0.0,0.0]$ \\
\hline $\begin{array}{l}3 \text { Hand oedema: difference } \\
\text { between hands in circumference } \\
(\mathrm{mm})\end{array}$ & 1 & & Mean Difference (IV, Fixed, 95\% CI) & Totals not selected \\
\hline 4 Complications & 1 & & Risk Ratio (M-H, Fixed, 95\% CI) & Totals not selected \\
\hline 4.1 Total complications & 1 & & Risk Ratio (M-H, Fixed, 95\% CI) & $0.0[0.0,0.0]$ \\
\hline 4.2 CRPS 1 (symptoms) & 1 & & Risk Ratio (M-H, Fixed, 95\% CI) & $0.0[0.0,0.0]$ \\
\hline 4.3 Median nerve compression & 1 & & Risk Ratio (M-H, Fixed, 95\% CI) & $0.0[0.0,0.0]$ \\
\hline 4.4 Finger stiffness & 1 & & Risk Ratio (M-H, Fixed, 95\% CI) & $0.0[0.0,0.0]$ \\
\hline 4.5 Adverse effect of PEMF & 1 & & Risk Ratio (M-H, Fixed, 95\% CI) & $0.0[0.0,0.0]$ \\
\hline
\end{tabular}

Comparison 5. Cross-education (strengthening exercises for opposite hand) versus no intervention (control)

\begin{tabular}{|c|c|c|c|c|}
\hline Outcome or subgroup title & $\begin{array}{l}\text { No. of } \\
\text { studies }\end{array}$ & $\begin{array}{c}\text { No. of } \\
\text { participants }\end{array}$ & Statistical method & Effect size \\
\hline $\begin{array}{l}1 \text { Patient-Rated Wrist Evaluation } \\
\text { (PRWE) (0 to 150: worst } \\
\text { results) }\end{array}$ & 1 & & Mean Difference (IV, Fixed, 95\% CI) & Totals not selected \\
\hline 1.1 At 9 weeks & 1 & & Mean Difference (IV, Fixed, 95\% CI) & $0.0[0.0,0.0]$ \\
\hline 1.2 At 12 weeks & 1 & & Mean Difference (IV, Fixed, 95\% CI) & $0.0[0.0,0.0]$ \\
\hline 1.3 At 26 weeks & 1 & & Mean Difference (IV, Fixed, 95\% CI) & $0.0[0.0,0.0]$ \\
\hline $\begin{array}{l}2 \text { Grip strength of fractured hand } \\
(\mathrm{kg})\end{array}$ & 1 & & Mean Difference (IV, Fixed, 95\% CI) & Totals not selected \\
\hline 2.1 At 9 weeks & 1 & & Mean Difference (IV, Fixed, 95\% CI) & $0.0[0.0,0.0]$ \\
\hline 2.2 At 12 weeks & 1 & & Mean Difference (IV, Fixed, 95\% CI) & $0.0[0.0,0.0]$ \\
\hline
\end{tabular}




\begin{tabular}{|c|c|c|c|}
\hline 2.3 At 26 weeks & 1 & Mean Difference (IV, Fixed, 95\% CI) & $0.0[0.0,0.0]$ \\
\hline $\begin{array}{l}3 \text { Range of motion: } \\
\text { supination/pronation (degrees) }\end{array}$ & 1 & Mean Difference (IV, Fixed, 95\% CI) & Totals not selected \\
\hline 3.1 At 9 weeks & 1 & Mean Difference (IV, Fixed, 95\% CI) & $0.0[0.0,0.0]$ \\
\hline 3.2 At 12 weeks & 1 & Mean Difference (IV, Fixed, 95\% CI) & $0.0[0.0,0.0]$ \\
\hline 3.3 At 26 weeks & 1 & Mean Difference (IV, Fixed, 95\% CI) & $0.0[0.0,0.0]$ \\
\hline $\begin{array}{l}4 \text { Range of motion: } \\
\text { flexion/extension (degrees) }\end{array}$ & 1 & Mean Difference (IV, Fixed, 95\% CI) & Totals not selected \\
\hline 4.1 At 9 weeks & 1 & Mean Difference (IV, Fixed, 95\% CI) & $0.0[0.0,0.0]$ \\
\hline 4.2 At 12 weeks & 1 & Mean Difference (IV, Fixed, 95\% CI) & $0.0[0.0,0.0]$ \\
\hline 4.3 At 26 weeks & 1 & Mean Difference (IV, Fixed, 95\% CI) & $0.0[0.0,0.0]$ \\
\hline
\end{tabular}

Comparison 6. Physiotherapy (one session for home exercises) versus no intervention (control) (post-immobilisation)

\begin{tabular}{|c|c|c|c|c|}
\hline Outcome or subgroup title & $\begin{array}{l}\text { No. of } \\
\text { studies }\end{array}$ & $\begin{array}{c}\text { No. of } \\
\text { participants }\end{array}$ & Statistical method & Effect size \\
\hline 1 PRWE scores at 3 and 6 weeks & 1 & & Mean Difference (IV, Fixed, 95\% CI) & Totals not selected \\
\hline $\begin{array}{l}1.1 \text { PRWE (pain) at } 3 \text { weeks } \\
\text { (0 to } 100 \text { : worst pain) }\end{array}$ & 1 & & Mean Difference (IV, Fixed, 95\% CI) & $0.0[0.0,0.0]$ \\
\hline $\begin{array}{l}1.2 \text { PRWE (function) at } \\
3 \text { weeks ( } 0 \text { to } 100 \text { : worst } \\
\text { function) }\end{array}$ & 1 & & Mean Difference (IV, Fixed, 95\% CI) & $0.0[0.0,0.0]$ \\
\hline $\begin{array}{l}1.3 \text { PRWE (pain) at } 6 \text { weeks } \\
\text { (0 to } 100: \text { worst pain) }\end{array}$ & 1 & & Mean Difference (IV, Fixed, 95\% CI) & $0.0[0.0,0.0]$ \\
\hline $\begin{array}{l}1.4 \text { PRWE (function) at } \\
6 \text { weeks ( } 0 \text { to } 100 \text { : worst } \\
\text { function) }\end{array}$ & 1 & & Mean Difference (IV, Fixed, 95\% CI) & $0.0[0.0,0.0]$ \\
\hline $\begin{array}{l}2 \text { QuickDASH scores at } 3 \text { and } 6 \\
\text { weeks }\end{array}$ & 1 & & Mean Difference (IV, Fixed, 95\% CI) & Totals not selected \\
\hline $\begin{array}{l}2.1 \text { QuickDASH (general) } \\
\text { at } 3 \text { weeks ( } 0 \text { to 100: worst } \\
\text { outcome) }\end{array}$ & 1 & & Mean Difference (IV, Fixed, 95\% CI) & $0.0[0.0,0.0]$ \\
\hline $\begin{array}{l}2.2 \text { QuickDASH (work) } \\
\text { at } 3 \text { weeks ( } 0 \text { to } 100 \text { : worst } \\
\text { outcome) }\end{array}$ & 1 & & Mean Difference (IV, Fixed, 95\% CI) & $0.0[0.0,0.0]$ \\
\hline $\begin{array}{l}2.3 \text { QuickDASH (sports) } \\
\text { at } 3 \text { weeks ( } 0 \text { to } 100 \text { : worst } \\
\text { outcome) }\end{array}$ & 1 & & Mean Difference (IV, Fixed, 95\% CI) & $0.0[0.0,0.0]$ \\
\hline $\begin{array}{l}2.4 \text { QuickDASH (general) } \\
\text { at } 6 \text { weeks ( } 0 \text { to } 100 \text { : worst } \\
\text { outcome) }\end{array}$ & 1 & & Mean Difference (IV, Fixed, 95\% CI) & $0.0[0.0,0.0]$ \\
\hline $\begin{array}{l}2.5 \text { QuickDASH (work) } \\
\text { at } 6 \text { weeks ( } 0 \text { to } 100 \text { : worst } \\
\text { outcome) }\end{array}$ & 1 & & Mean Difference (IV, Fixed, 95\% CI) & $0.0[0.0,0.0]$ \\
\hline $\begin{array}{l}\text { 2.6 QuickDASH (sports) } \\
\text { at } 6 \text { weeks ( } 0 \text { to } 100 \text { : worst } \\
\text { outcome) }\end{array}$ & 1 & & Mean Difference (IV, Fixed, 95\% CI) & $0.0[0.0,0.0]$ \\
\hline
\end{tabular}

Rehabilitation for distal radial fractures in adults (Review)

Copyright $\odot 2015$ The Cochrane Collaboration. Published by John Wiley \& Sons, Ltd. 


\begin{tabular}{l} 
3 Grip strength $(\mathrm{kg})$ at 3 and 6 \\
weeks \\
3.1 At 3 weeks \\
3.2 At 6 weeks \\
4 Range of motion at 6 weeks \\
(degrees) \\
4.1 Extension \\
4.2 Flexion \\
4.3 Radial deviation \\
4.4 Ulnar deviation \\
4.5 Pronation \\
4.6 Supination \\
5 Change in range of motion at 6 \\
weeks (degrees) \\
5.1 Extension \\
5.2 Flexion \\
5.3 Radial deviation \\
5.4 Ulnar deviation \\
5.5 Pronation \\
5.6 Supination \\
6 Complications \\
7 Request for more physiotherapy \\
\hline
\end{tabular}

\author{
Mean Difference (IV, Fixed, 95\% CI) \\ Mean Difference (IV, Fixed, 95\% CI) \\ Mean Difference (IV, Fixed, 95\% CI) \\ Mean Difference (IV, Fixed, 95\% CI) \\ Mean Difference (IV, Fixed, 95\% CI) \\ Mean Difference (IV, Fixed, 95\% CI) \\ Mean Difference (IV, Fixed, 95\% CI) \\ Mean Difference (IV, Fixed, 95\% CI) \\ Mean Difference (IV, Fixed, 95\% CI) \\ Mean Difference (IV, Fixed, 95\% CI) \\ Mean Difference (IV, Fixed, 95\% CI) \\ Mean Difference (IV, Fixed, 95\% CI) \\ Mean Difference (IV, Fixed, 95\% CI) \\ Mean Difference (IV, Fixed, 95\% CI) \\ Mean Difference (IV, Fixed, 95\% CI) \\ Mean Difference (IV, Fixed, 95\% CI) \\ Mean Difference (IV, Fixed, 95\% CI) \\ Risk Ratio (M-H, Fixed, 95\% CI) \\ Risk Ratio (M-H, Fixed, 95\% CI)
}

Totals not selected

$0.0[0.0,0.0]$

$0.0[0.0,0.0]$

Totals not selected

$0.0[0.0,0.0]$

$0.0[0.0,0.0]$

$0.0[0.0,0.0]$

$0.0[0.0,0.0]$

$0.0[0.0,0.0]$

$0.0[0.0,0.0]$

Totals not selected

$0.0[0.0,0.0]$

$0.0[0.0,0.0]$

$0.0[0.0,0.0]$

$0.0[0.0,0.0]$

$0.0[0.0,0.0]$

$0.0[0.0,0.0]$

Totals not selected

Totals not selected

\title{
Comparison 7. Post-immobilisation occupational or physiotherapy versus no intervention (control)
}

$\begin{array}{lllll}\text { Outcome or subgroup title } & \begin{array}{l}\text { No. of } \\ \text { studies }\end{array} & \begin{array}{c}\text { No. of } \\ \text { participants }\end{array} & \text { Statistical method } & \text { Effect size }\end{array}$

\begin{tabular}{|c|c|c|c|}
\hline $\begin{array}{l}1 \text { Patient-Rated Wrist Evaluation } \\
\text { (PRWE) at } 24 \text { weeks (\%: } 100 \% \\
\text { = worst results) }\end{array}$ & 1 & Mean Difference (IV, Fixed, 95\% CI) & Totals not selected \\
\hline 1.1 Overall result & 1 & Mean Difference (IV, Fixed, 95\% CI) & $0.0[0.0,0.0]$ \\
\hline 1.2 Pain items & 1 & Mean Difference (IV, Fixed, 95\% CI) & $0.0[0.0,0.0]$ \\
\hline 1.3 Activity items & 1 & Mean Difference (IV, Fixed, 95\% CI) & $0.0[0.0,0.0]$ \\
\hline 1.4 Disability items & 1 & Mean Difference (IV, Fixed, 95\% CI) & $0.0[0.0,0.0]$ \\
\hline $\begin{array}{l}2 \text { Activities of daily living scores } \\
\text { (\% of unaffected side) }\end{array}$ & 1 & Mean Difference (IV, Fixed, 95\% CI) & Totals not selected \\
\hline 2.13 months & 1 & Mean Difference (IV, Fixed, 95\% CI) & $0.0[0.0,0.0]$ \\
\hline 2.26 months & 1 & Mean Difference (IV, Fixed, 95\% CI) & $0.0[0.0,0.0]$ \\
\hline 3 Grip strength $(\mathrm{kg})$ & 2 & Mean Difference (IV, Fixed, 95\% CI) & Totals not selected \\
\hline 3.13 months & 1 & Mean Difference (IV, Fixed, 95\% CI) & $0.0[0.0,0.0]$ \\
\hline 3.224 weeks & 1 & Mean Difference (IV, Fixed, 95\% CI) & $0.0[0.0,0.0]$ \\
\hline 3.39 months & 1 & Mean Difference (IV, Fixed, 95\% CI) & $0.0[0.0,0.0]$ \\
\hline $\begin{array}{l}4 \text { Grip strength (\% of unaffected } \\
\text { side) }\end{array}$ & 1 & Mean Difference (IV, Fixed, 95\% CI) & Totals not selected \\
\hline 4.13 months & 1 & Mean Difference (IV, Fixed, 95\% CI) & $0.0[0.0,0.0]$ \\
\hline 4.26 months & 1 & Mean Difference (IV, Fixed, 95\% CI) & $0.0[0.0,0.0]$ \\
\hline $\begin{array}{l}5 \text { Pain (VAS: none to worst } \\
\text { imaginable at } 10 \mathrm{~cm} \text { ) }\end{array}$ & 1 & Mean Difference (IV, Fixed, 95\% CI) & Totals not selected \\
\hline 5.13 months & 1 & Mean Difference (IV, Fixed, 95\% CI) & $0.0[0.0,0.0]$ \\
\hline
\end{tabular}

Rehabilitation for distal radial fractures in adults (Review)

Copyright $\odot 2015$ The Cochrane Collaboration. Published by John Wiley \& Sons, Ltd. 


\begin{tabular}{|c|c|c|c|}
\hline 5.26 months & 1 & Mean Difference (IV, Fixed, 95\% CI) & $0.0[0.0,0.0]$ \\
\hline 6 Range of motion at 24 weeks & 1 & Mean Difference (IV, Fixed, 95\% CI) & Totals not selected \\
\hline 6.1 Flexion (degrees) & 1 & Mean Difference (IV, Fixed, 95\% CI) & $0.0[0.0,0.0]$ \\
\hline 6.2 Extension (degrees) & 1 & Mean Difference (IV, Fixed, 95\% CI) & $0.0[0.0,0.0]$ \\
\hline $\begin{array}{l}7 \text { Range of motion ( } \% \text { of } \\
\text { unaffected side) at } 3 \text { months }\end{array}$ & 1 & Mean Difference (IV, Fixed, 95\% CI) & Totals not selected \\
\hline 7.1 Pronation-supination & 1 & Mean Difference (IV, Fixed, 95\% CI) & $0.0[0.0,0.0]$ \\
\hline 7.2 Flexion-extension & 1 & Mean Difference (IV, Fixed, 95\% CI) & $0.0[0.0,0.0]$ \\
\hline 7.3 Radial-ulnar deviation & 1 & Mean Difference (IV, Fixed, 95\% CI) & $0.0[0.0,0.0]$ \\
\hline $\begin{array}{l}8 \text { Range of motion ( } \% \text { of } \\
\text { unaffected side) at } 6 \text { months }\end{array}$ & 1 & Mean Difference (IV, Fixed, 95\% CI) & Totals not selected \\
\hline 8.1 Pronation-supination & 1 & Mean Difference (IV, Fixed, 95\% CI) & $0.0[0.0,0.0]$ \\
\hline 8.2 Flexion-extension & 1 & Mean Difference (IV, Fixed, 95\% CI) & $0.0[0.0,0.0]$ \\
\hline 8.3 Radial-ulnar deviation & 1 & Mean Difference (IV, Fixed, 95\% CI) & $0.0[0.0,0.0]$ \\
\hline 9 Number of treatments & 1 & Mean Difference (IV, Fixed, 95\% CI) & Totals not selected \\
\hline 10 Complications & 1 & Risk Ratio (M-H, Fixed, 95\% CI) & Totals not selected \\
\hline 10.1 CRPS-1 & 1 & Risk Ratio (M-H, Fixed, 95\% CI) & $0.0[0.0,0.0]$ \\
\hline 10.2 Carpal tunnel syndrome & 1 & Risk Ratio (M-H, Fixed, 95\% CI) & $0.0[0.0,0.0]$ \\
\hline
\end{tabular}

Comparison 8. Continuous passive motion (CPM) (post-external fixation) versus no intervention (control)

\begin{tabular}{lcccc} 
Outcome or subgroup title & $\begin{array}{c}\text { No. of } \\
\text { studies }\end{array}$ & $\begin{array}{c}\text { No. of } \\
\text { participants }\end{array}$ & Statistical method & Effect size \\
\hline $\begin{array}{l}1 \text { Time to recover independence } \\
\text { (weeks) }\end{array}$ & 1 & Mean Difference (IV, Fixed, 95\% CI) & Totals not selected \\
\hline
\end{tabular}

\section{Comparison 9. Pulsed electromagnetic field (PEMF) (post-immobilisation) versus sham control}

\begin{tabular}{|c|c|c|c|c|}
\hline Outcome or subgroup title & $\begin{array}{l}\text { No. of } \\
\text { studies }\end{array}$ & $\begin{array}{c}\text { No. of } \\
\text { participants }\end{array}$ & Statistical method & Effect size \\
\hline 1 Pain and volume at day 5 & 1 & & Mean Difference (IV, Fixed, 95\% CI) & Totals not selected \\
\hline $\begin{array}{l}\text { 1.1 Pain (scale } 0 \text { : no pain } \\
\text { to } 10 \mathrm{~cm} \text { : worst imaginable) } \\
\text { during active wrist movements }\end{array}$ & 1 & & Mean Difference (IV, Fixed, 95\% CI) & $0.0[0.0,0.0]$ \\
\hline 1.2 Volume $(\mathrm{ml})$ & 1 & & Mean Difference (IV, Fixed, 95\% CI) & $0.0[0.0,0.0]$ \\
\hline 2 Range of motion at day 5 & 1 & & Mean Difference (IV, Fixed, 95\% CI) & Totals not selected \\
\hline 2.1 Pronation (degrees) & 1 & & Mean Difference (IV, Fixed, 95\% CI) & $0.0[0.0,0.0]$ \\
\hline 2.2 Supination (degrees) & 1 & & Mean Difference (IV, Fixed, 95\% CI) & $0.0[0.0,0.0]$ \\
\hline 2.3 Flexion (degrees) & 1 & & Mean Difference (IV, Fixed, 95\% CI) & $0.0[0.0,0.0]$ \\
\hline 2.4 Extension (degrees) & 1 & & Mean Difference (IV, Fixed, 95\% CI) & $0.0[0.0,0.0]$ \\
\hline 2.5 Radial deviation (degrees) & 1 & & Mean Difference (IV, Fixed, 95\% CI) & $0.0[0.0,0.0]$ \\
\hline 2.6 Ulnar deviation (degrees) & 1 & & Mean Difference (IV, Fixed, 95\% CI) & $0.0[0.0,0.0]$ \\
\hline
\end{tabular}

Rehabilitation for distal radial fractures in adults (Review) 


\begin{tabular}{|c|c|c|c|c|}
\hline Outcome or subgroup title & $\begin{array}{l}\text { No. of } \\
\text { studies }\end{array}$ & $\begin{array}{c}\text { No. of } \\
\text { participants }\end{array}$ & Statistical method & Effect size \\
\hline 1 Pain and volume at day 5 & 1 & & Mean Difference (IV, Fixed, 95\% CI) & Totals not selected \\
\hline $\begin{array}{l}1.1 \text { Pain (scale } 0: \text { no pain } \\
\text { to } 10 \mathrm{~cm} \text { : worst imaginable) } \\
\text { during active wrist movements }\end{array}$ & 1 & & Mean Difference (IV, Fixed, 95\% CI) & $0.0[0.0,0.0]$ \\
\hline 1.2 Volume $(\mathrm{ml})$ & 1 & & Mean Difference (IV, Fixed, 95\% CI) & $0.0[0.0,0.0]$ \\
\hline 2 Range of motion at day 5 & 1 & & Mean Difference (IV, Fixed, 95\% CI) & Totals not selected \\
\hline 2.1 Pronation (degrees) & 1 & & Mean Difference (IV, Fixed, 95\% CI) & $0.0[0.0,0.0]$ \\
\hline 2.2 Supination (degrees) & 1 & & Mean Difference (IV, Fixed, 95\% CI) & $0.0[0.0,0.0]$ \\
\hline 2.3 Flexion (degrees) & 1 & & Mean Difference (IV, Fixed, 95\% CI) & $0.0[0.0,0.0]$ \\
\hline 2.4 Extension (degrees) & 1 & & Mean Difference (IV, Fixed, 95\% CI) & $0.0[0.0,0.0]$ \\
\hline 2.5 Radial deviation (degrees) & 1 & & Mean Difference (IV, Fixed, 95\% CI) & $0.0[0.0,0.0]$ \\
\hline 2.6 Ulnar deviation (degrees) & 1 & & Mean Difference (IV, Fixed, 95\% CI) & $0.0[0.0,0.0]$ \\
\hline
\end{tabular}

Comparison 11. Pulsed electromagnetic field (PEMF) plus ice (post-immobilisation) versus no intervention (control)

\begin{tabular}{|c|c|c|c|c|}
\hline Outcome or subgroup title & $\begin{array}{l}\text { No. of } \\
\text { studies }\end{array}$ & $\begin{array}{c}\text { No. of } \\
\text { participants }\end{array}$ & Statistical method & Effect size \\
\hline 1 Pain and volume at day 5 & 1 & & Mean Difference (IV, Fixed, 95\% CI) & Totals not selected \\
\hline $\begin{array}{l}\text { 1.1 Pain (scale } 0: \text { no pain } \\
\text { to } 10 \mathrm{~cm} \text { : worst imaginable) } \\
\text { during active wrist movements }\end{array}$ & 1 & & Mean Difference (IV, Fixed, 95\% CI) & $0.0[0.0,0.0]$ \\
\hline 1.2 Volume $(\mathrm{ml})$ & 1 & & Mean Difference (IV, Fixed, 95\% CI) & $0.0[0.0,0.0]$ \\
\hline 2 Range of motion at day 5 & 1 & & Mean Difference (IV, Fixed, 95\% CI) & Totals not selected \\
\hline 2.1 Pronation (degrees) & 1 & & Mean Difference (IV, Fixed, 95\% CI) & $0.0[0.0,0.0]$ \\
\hline 2.2 Supination (degrees) & 1 & & Mean Difference (IV, Fixed, 95\% CI) & $0.0[0.0,0.0]$ \\
\hline 2.3 Flexion (degrees) & 1 & & Mean Difference (IV, Fixed, 95\% CI) & $0.0[0.0,0.0]$ \\
\hline 2.4 Extension (degrees) & 1 & & Mean Difference (IV, Fixed, 95\% CI) & $0.0[0.0,0.0]$ \\
\hline 2.5 Radial deviation (degrees) & 1 & & Mean Difference (IV, Fixed, 95\% CI) & $0.0[0.0,0.0]$ \\
\hline 2.6 Ulnar deviation (degrees) & 1 & & Mean Difference (IV, Fixed, 95\% CI) & $0.0[0.0,0.0]$ \\
\hline
\end{tabular}




\begin{tabular}{|c|c|c|c|c|}
\hline Outcome or subgroup title & $\begin{array}{l}\text { No. of } \\
\text { studies }\end{array}$ & $\begin{array}{c}\text { No. of } \\
\text { participants }\end{array}$ & Statistical method & Effect size \\
\hline 1 Grip strength $(\mathrm{kg})$ at 6 weeks & 1 & & Mean Difference (IV, Fixed, 95\% CI) & Totals not selected \\
\hline 2 Range of motion at 6 weeks & 1 & & Mean Difference (IV, Fixed, 95\% CI) & Totals not selected \\
\hline 2.1 Pronation (degrees) & 1 & & Mean Difference (IV, Fixed, 95\% CI) & $0.0[0.0,0.0]$ \\
\hline 2.2 Supination (degrees) & 1 & & Mean Difference (IV, Fixed, 95\% CI) & $0.0[0.0,0.0]$ \\
\hline 2.3 Flexion (degrees) & 1 & & Mean Difference (IV, Fixed, 95\% CI) & $0.0[0.0,0.0]$ \\
\hline 2.4 Extension (degrees) & 1 & & Mean Difference (IV, Fixed, 95\% CI) & $0.0[0.0,0.0]$ \\
\hline 2.5 Radial deviation (degrees) & 1 & & Mean Difference (IV, Fixed, 95\% CI) & $0.0[0.0,0.0]$ \\
\hline 2.6 Ulnar deviation (degrees) & 1 & & Mean Difference (IV, Fixed, 95\% CI) & $0.0[0.0,0.0]$ \\
\hline $\begin{array}{l}3 \text { Web space angle (degrees) at } 6 \\
\text { weeks }\end{array}$ & 1 & & Mean Difference (IV, Fixed, 95\% CI) & Totals not selected \\
\hline $\begin{array}{l}4 \text { Wrist extension at discharge (4 } \\
\text { weeks) }\end{array}$ & 1 & & Mean Difference (IV, Fixed, 95\% CI) & Totals not selected \\
\hline 5 Number of treatments & 2 & & Mean Difference (IV, Fixed, 95\% CI) & Totals not selected \\
\hline 6 Time to discharge (days) & 1 & & Mean Difference (IV, Fixed, 95\% CI) & Totals not selected \\
\hline 7 Complications at 6 weeks & 1 & & Risk Ratio (M-H, Fixed, 95\% CI) & Totals not selected \\
\hline 7.1 Carpal tunnel syndrome & 1 & & Risk Ratio (M-H, Fixed, 95\% CI) & $0.0[0.0,0.0]$ \\
\hline $\begin{array}{l}7.2 \text { Finger stiffness } \\
\text { (continuing) }\end{array}$ & 1 & & Risk Ratio (M-H, Fixed, 95\% CI) & $0.0[0.0,0.0]$ \\
\hline $\begin{array}{l}7.3 \text { Complex regional pain } \\
\text { syndrome (continuing) }\end{array}$ & 1 & & Risk Ratio (M-H, Fixed, 95\% CI) & $0.0[0.0,0.0]$ \\
\hline 7.4 Malunion & 1 & & Risk Ratio (M-H, Fixed, 95\% CI) & $0.0[0.0,0.0]$ \\
\hline
\end{tabular}

Comparison 13. Low frequency, long-wave ultrasound (post-immobilisation) versus sham intervention

\begin{tabular}{|c|c|c|c|c|}
\hline Outcome or subgroup title & $\begin{array}{l}\text { No. of } \\
\text { studies }\end{array}$ & $\begin{array}{c}\text { No. of } \\
\text { participants }\end{array}$ & Statistical method & Effect size \\
\hline $\begin{array}{l}1 \text { Greater than } 30 \% \text { loss of wrist } \\
\text { motion (flexion-extension) at } 8 \\
\text { weeks }\end{array}$ & 1 & & Risk Ratio (M-H, Fixed, 95\% CI) & Totals not selected \\
\hline 2 Referral for physiotherapy & 1 & & Risk Ratio (M-H, Fixed, 95\% CI) & Totals not selected \\
\hline
\end{tabular}




\begin{tabular}{|c|c|c|c|c|}
\hline Outcome or subgroup title & $\begin{array}{l}\text { No. of } \\
\text { studies }\end{array}$ & $\begin{array}{c}\text { No. of } \\
\text { participants }\end{array}$ & Statistical method & Effect size \\
\hline $\begin{array}{l}1 \text { Grip strength at end of treatment } \\
(\mathrm{kg})\end{array}$ & 1 & & Mean Difference (IV, Fixed, 95\% CI) & Totals not selected \\
\hline $\begin{array}{l}2 \text { Pain (scale } 0 \text { : no pain to } \\
\text { 5: excruciating) at end of } \\
\text { treatment }\end{array}$ & 1 & & Mean Difference (IV, Fixed, 95\% CI) & Totals not selected \\
\hline $\begin{array}{l}3 \text { Range of motion at end of } \\
\text { treatment }\end{array}$ & 1 & & Mean Difference (IV, Fixed, 95\% CI) & Totals not selected \\
\hline 3.1 Pronation (degrees) & 1 & & Mean Difference (IV, Fixed, 95\% CI) & $0.0[0.0,0.0]$ \\
\hline 3.2 Supination (degrees) & 1 & & Mean Difference (IV, Fixed, 95\% CI) & $0.0[0.0,0.0]$ \\
\hline 3.3 Flexion (degrees) & 1 & & Mean Difference (IV, Fixed, 95\% CI) & $0.0[0.0,0.0]$ \\
\hline 3.4 Extension (degrees) & 1 & & Mean Difference (IV, Fixed, 95\% CI) & $0.0[0.0,0.0]$ \\
\hline 3.5 Radial deviation (degrees) & 1 & & Mean Difference (IV, Fixed, 95\% CI) & $0.0[0.0,0.0]$ \\
\hline 3.6 Ulnar deviation (degrees) & 1 & & Mean Difference (IV, Fixed, 95\% CI) & $0.0[0.0,0.0]$ \\
\hline 4 Finger flexion at end of treatment & 1 & & Mean Difference (IV, Fixed, 95\% CI) & Totals not selected \\
\hline $\begin{array}{l}\text { 4.1 Thumb MCP (metacarpal } \\
\text { phalange) flexion (degrees) }\end{array}$ & 1 & & Mean Difference (IV, Fixed, 95\% CI) & $0.0[0.0,0.0]$ \\
\hline $\begin{array}{l}4.2 \text { Index finger MCP flexion } \\
\text { (degrees) }\end{array}$ & 1 & & Mean Difference (IV, Fixed, 95\% CI) & $0.0[0.0,0.0]$ \\
\hline $\begin{array}{l}4.3 \text { Long finger MCP flexion } \\
\text { (degrees) }\end{array}$ & 1 & & Mean Difference (IV, Fixed, 95\% CI) & $0.0[0.0,0.0]$ \\
\hline $\begin{array}{l}\text { 4.4 Ring finger MCL flexion } \\
\text { (degrees) }\end{array}$ & 1 & & Mean Difference (IV, Fixed, 95\% CI) & $0.0[0.0,0.0]$ \\
\hline $\begin{array}{l}\text { 4.5 Little finger MCP flexion } \\
\text { (degrees) }\end{array}$ & 1 & & Mean Difference (IV, Fixed, 95\% CI) & $0.0[0.0,0.0]$ \\
\hline 5 Oedema $(\mathrm{ml})$ & 1 & & Mean Difference (IV, Fixed, 95\% CI) & Totals not selected \\
\hline $\begin{array}{l}5.1 \text { Short term: at end of each } \\
\text { session }\end{array}$ & 1 & & Mean Difference (IV, Fixed, 95\% CI) & $0.0[0.0,0.0]$ \\
\hline 5.2 At end of treatment & 1 & & Mean Difference (IV, Fixed, 95\% CI) & $0.0[0.0,0.0]$ \\
\hline
\end{tabular}

Comparison 15. Dynamic wrist extension splint versus no intervention (control) (post-immobilisation)

\begin{tabular}{|c|c|c|c|c|}
\hline Outcome or subgroup title & $\begin{array}{l}\text { No. of } \\
\text { studies }\end{array}$ & $\begin{array}{c}\text { No. of } \\
\text { participants }\end{array}$ & Statistical method & Effect size \\
\hline $\begin{array}{l}1 \text { Patient-Rated Wrist Evaluation } \\
\text { (PRWE) }(\%: 100 \%=\text { worst } \\
\text { results) }\end{array}$ & 1 & & Mean Difference (IV, Fixed, 95\% CI) & Totals not selected \\
\hline $\begin{array}{l}1.1 \text { At } 8 \text { weeks (end of } \\
\text { treatment) }\end{array}$ & 1 & & Mean Difference (IV, Fixed, 95\% CI) & $0.0[0.0,0.0]$ \\
\hline 1.2 At 12 weeks & 1 & & Mean Difference (IV, Fixed, 95\% CI) & $0.0[0.0,0.0]$ \\
\hline $\begin{array}{l}1.3 \text { At } 12 \text { weeks (per-protocol } \\
\text { analysis) }\end{array}$ & 1 & & Mean Difference (IV, Fixed, 95\% CI) & $0.0[0.0,0.0]$ \\
\hline
\end{tabular}

Rehabilitation for distal radial fractures in adults (Review) 
Performance Measure at 12

weeks

2.1 Ability to perform the key

1 activity (10 points maximum)

2.2 Satisfaction with ability to perform key activity (10 points $\max )$

3 Range of motion at 12 weeks

3.1 Passive wrist extension

(degrees)

3.2 Active wrist extension

(degrees)

3.3 Active wrist flexion

(degrees)

3.4 Active radial deviation

(degrees)

3.5 Active ulnar deviation

(degrees)
Mean Difference (IV, Fixed, 95\% CI)

Mean Difference (IV, Fixed, 95\% CI)

Mean Difference (IV, Fixed, 95\% CI)

Mean Difference (IV, Fixed, 95\% CI)

Mean Difference (IV, Fixed, 95\% CI)

Mean Difference (IV, Fixed, 95\% CI)

Mean Difference (IV, Fixed, 95\% CI)

Mean Difference (IV, Fixed, 95\% CI)
$0.0[0.0,0.0]$

$0.0[0.0,0.0]$

Totals not selected

$0.0[0.0,0.0]$

$0.0[0.0,0.0]$

$0.0[0.0,0.0]$

$0.0[0.0,0.0]$

$0.0[0.0,0.0]$

Comparison 16. Post-immobilisation physiotherapy versus instructions from physician

\begin{tabular}{|c|c|c|c|c|}
\hline Outcome or subgroup title & $\begin{array}{l}\text { No. of } \\
\text { studies }\end{array}$ & $\begin{array}{c}\text { No. of } \\
\text { participants }\end{array}$ & Statistical method & Effect size \\
\hline $\begin{array}{l}1 \text { Wrist extension (degrees) at } 6 \\
\text { weeks }\end{array}$ & 1 & & Mean Difference (IV, Fixed, 95\% CI) & Totals not selected \\
\hline
\end{tabular}

\section{Comparison 17. Pulsed electromagnetic field (PEMF) versus ice (post-immobilisation)}

\begin{tabular}{|c|c|c|c|c|}
\hline Outcome or subgroup title & $\begin{array}{l}\text { No. of } \\
\text { studies }\end{array}$ & $\begin{array}{c}\text { No. of } \\
\text { participants }\end{array}$ & Statistical method & Effect size \\
\hline 1 Pain and volume at day 5 & 1 & & Mean Difference (IV, Fixed, 95\% CI) & Totals not selected \\
\hline $\begin{array}{l}1.1 \text { Pain (scale } 0: \text { no pain } \\
\text { to } 10 \mathrm{~cm} \text { : worst imaginable) } \\
\text { during active wrist movements }\end{array}$ & 1 & & Mean Difference (IV, Fixed, 95\% CI) & $0.0[0.0,0.0]$ \\
\hline 1.2 Volume $(\mathrm{ml})$ & 1 & & Mean Difference (IV, Fixed, 95\% CI) & $0.0[0.0,0.0]$ \\
\hline 2 Range of motion at day 5 & 1 & & Mean Difference (IV, Fixed, 95\% CI) & Totals not selected \\
\hline 2.1 Pronation (degrees) & 1 & & Mean Difference (IV, Fixed, 95\% CI) & $0.0[0.0,0.0]$ \\
\hline 2.2 Supination (degrees) & 1 & & Mean Difference (IV, Fixed, 95\% CI) & $0.0[0.0,0.0]$ \\
\hline 2.3 Flexion (degrees) & 1 & & Mean Difference (IV, Fixed, 95\% CI) & $0.0[0.0,0.0]$ \\
\hline 2.4 Extension (degrees) & 1 & & Mean Difference (IV, Fixed, 95\% CI) & $0.0[0.0,0.0]$ \\
\hline 2.5 Radial deviation (degrees) & 1 & & Mean Difference (IV, Fixed, 95\% CI) & $0.0[0.0,0.0]$ \\
\hline 2.6 Ulnar deviation (degrees) & 1 & & Mean Difference (IV, Fixed, 95\% CI) & $0.0[0.0,0.0]$ \\
\hline
\end{tabular}

Rehabilitation for distal radial fractures in adults (Review)

Copyright $\odot 2015$ The Cochrane Collaboration. Published by John Wiley \& Sons, Ltd. 


\begin{tabular}{|c|c|c|c|c|}
\hline Outcome or subgroup title & $\begin{array}{l}\text { No. of } \\
\text { studies }\end{array}$ & $\begin{array}{c}\text { No. of } \\
\text { participants }\end{array}$ & Statistical method & Effect size \\
\hline $\begin{array}{l}\text { Canadian Occupational } \\
\text { Performance Measure at } 9 \\
\text { weeks (clinically important } \\
\text { improvement) }\end{array}$ & 1 & & Risk Ratio (M-H, Fixed, 95\% CI) & Totals not selected \\
\hline $\begin{array}{l}1.1 \text { Ability to perform the key } \\
\text { activity (change } 2+\text { in score) }\end{array}$ & 1 & & Risk Ratio (M-H, Fixed, 95\% CI) & $0.0[0.0,0.0]$ \\
\hline $\begin{array}{l}1.2 \text { Satisfaction with ability to } \\
\text { perform key activity (change } \\
2+\text { in score) }\end{array}$ & 1 & & Risk Ratio (M-H, Fixed, 95\% CI) & $0.0[0.0,0.0]$ \\
\hline 2 Pain (VAS: 0 to $100:$ worst pain) & 1 & & Mean Difference (IV, Fixed, 95\% CI) & Totals not selected \\
\hline 2.1 Pain at rest (9 weeks) & 1 & & Mean Difference (IV, Fixed, 95\% CI) & $0.0[0.0,0.0]$ \\
\hline 2.2 Pain at rest (26 weeks) & 1 & & Mean Difference (IV, Fixed, 95\% CI) & $0.0[0.0,0.0]$ \\
\hline 2.3 Pain when active ( 9 weeks) & 1 & & Mean Difference (IV, Fixed, 95\% CI) & $0.0[0.0,0.0]$ \\
\hline $\begin{array}{l}2.4 \text { Pain when active }(26 \\
\text { weeks) }\end{array}$ & 1 & & Mean Difference (IV, Fixed, 95\% CI) & $0.0[0.0,0.0]$ \\
\hline 3 Complications & 1 & & Risk Ratio (M-H, Fixed, 95\% CI) & Totals not selected \\
\hline $\begin{array}{l}4 \text { Number of occupational therapy } \\
\text { sessions }\end{array}$ & 1 & & Mean Difference (IV, Fixed, 95\% CI) & Totals not selected \\
\hline $\begin{array}{l}5 \text { Receiving oedema treatment } \\
\text { after scheduled period }\end{array}$ & 1 & & Risk Ratio (M-H, Fixed, 95\% CI) & Totals not selected \\
\hline $\begin{array}{l}5.1 \text { Receiving oedema } \\
\text { treatment after } 6 \text { weeks }\end{array}$ & 1 & & Risk Ratio (M-H, Fixed, 95\% CI) & $0.0[0.0,0.0]$ \\
\hline $\begin{array}{l}5.2 \text { Receiving oedema } \\
\text { treatment after } 9 \text { weeks }\end{array}$ & 1 & & Risk Ratio (M-H, Fixed, 95\% CI) & $0.0[0.0,0.0]$ \\
\hline $\begin{array}{l}6 \text { Oedema: volume difference } \\
\text { between injured and } \\
\text { non-injured side }(\mathrm{mL})\end{array}$ & 1 & & Mean Difference (IV, Fixed, 95\% CI) & Totals not selected \\
\hline 6.1 Oedema (9 weeks) & 1 & & Mean Difference (IV, Fixed, 95\% CI) & $0.0[0.0,0.0]$ \\
\hline 6.2 Oedema (26 weeks) & 1 & & Mean Difference (IV, Fixed, 95\% CI) & $0.0[0.0,0.0]$ \\
\hline
\end{tabular}

Comparison 19. Supervised training by physiotherapist versus instructions by physician (from definitive treatment)

\begin{tabular}{|c|c|c|c|c|}
\hline Outcome or subgroup title & $\begin{array}{l}\text { No. of } \\
\text { studies }\end{array}$ & $\begin{array}{c}\text { No. of } \\
\text { participants }\end{array}$ & Statistical method & Effect size \\
\hline 1 Strength and power at 12 weeks & 1 & & Mean Difference (IV, Fixed, 95\% CI) & Totals not selected \\
\hline 1.1 "Grip strength" (kg/cm2) & 1 & & Mean Difference (IV, Fixed, 95\% CI) & $0.0[0.0,0.0]$ \\
\hline $\begin{array}{l}1.2 \mathrm{Hand} \text { pumping power } \\
(\mathrm{mmHg})\end{array}$ & 1 & & Mean Difference (IV, Fixed, 95\% CI) & $0.0[0.0,0.0]$ \\
\hline 2 Range of motion at 12 weeks & 1 & & Mean Difference (IV, Fixed, 95\% CI) & Totals not selected \\
\hline 2.1 Pronation (degrees) & 1 & & Mean Difference (IV, Fixed, 95\% CI) & $0.0[0.0,0.0]$ \\
\hline 2.2 Supination (degrees) & 1 & & Mean Difference (IV, Fixed, 95\% CI) & $0.0[0.0,0.0]$ \\
\hline
\end{tabular}

Rehabilitation for distal radial fractures in adults (Review)

Copyright $\odot 2015$ The Cochrane Collaboration. Published by John Wiley \& Sons, Ltd. 
2.3 Flexion (degrees)

2.4 Extension (degrees)

2.5 Radial deviation (degrees)

2.6 Ulnar deviation (degrees)
Mean Difference (IV, Fixed, 95\% CI)

Mean Difference (IV, Fixed, 95\% CI)

Mean Difference (IV, Fixed, 95\% CI)

Mean Difference (IV, Fixed, 95\% CI)
$0.0[0.0,0.0]$

$0.0[0.0,0.0]$

$0.0[0.0,0.0]$

$0.0[0.0,0.0]$

Comparison 20. Physiotherapy/occupational therapy versus home exercise programme (post-surgery)

\begin{tabular}{|c|c|c|c|c|}
\hline Outcome or subgroup title & $\begin{array}{l}\text { No. of } \\
\text { studies }\end{array}$ & $\begin{array}{c}\text { No. of } \\
\text { participants }\end{array}$ & Statistical method & Effect size \\
\hline $\begin{array}{l}1 \text { PRWE scores at } 6 \text { weeks (0: } \\
\text { normal to } 150 \text { : worst outcome) }\end{array}$ & 1 & & Mean Difference (IV, Fixed, 95\% CI) & Totals not selected \\
\hline $\begin{array}{l}2 \text { DASH score (0 to 100: higher } \\
\text { scores = worse upper-extremity } \\
\text { function) }\end{array}$ & 1 & & Mean Difference (IV, Fixed, 95\% CI) & Totals not selected \\
\hline 2.1 DASH scores at 3 months & 1 & & Mean Difference (IV, Fixed, 95\% CI) & $0.0[0.0,0.0]$ \\
\hline 2.2 DASH scores at 6 months & 1 & & Mean Difference (IV, Fixed, 95\% CI) & $0.0[0.0,0.0]$ \\
\hline $\begin{array}{l}3 \text { Mayo wrist score ( } 0 \text { to } 100 \text { : } \\
\text { higher scores = best functional } \\
\text { outcome) }\end{array}$ & 1 & & Mean Difference (IV, Fixed, 95\% CI) & Totals not selected \\
\hline 3.1 Mayo scores at 3 months & 1 & & Mean Difference (IV, Fixed, 95\% CI) & $0.0[0.0,0.0]$ \\
\hline 3.2 Mayo scores at 6 months & 1 & & Mean Difference (IV, Fixed, 95\% CI) & $0.0[0.0,0.0]$ \\
\hline $\begin{array}{l}4 \text { Pain at rest (VAS: } 0 \text { to } 10: \text { worst } \\
\text { pain) }\end{array}$ & 1 & & Mean Difference (IV, Fixed, 95\% CI) & Totals not selected \\
\hline 4.1 Pain at 3 months & 1 & & Mean Difference (IV, Fixed, 95\% CI) & $0.0[0.0,0.0]$ \\
\hline 4.2 Pain at 6 months & 1 & & Mean Difference (IV, Fixed, 95\% CI) & $0.0[0.0,0.0]$ \\
\hline 5 Grip strength (kg) & 1 & & Mean Difference (IV, Fixed, 95\% CI) & Totals not selected \\
\hline 5.1 Grip strength at 3 months & 1 & & Mean Difference (IV, Fixed, 95\% CI) & $0.0[0.0,0.0]$ \\
\hline 5.2 Grip strength at 6 months & 1 & & Mean Difference (IV, Fixed, 95\% CI) & $0.0[0.0,0.0]$ \\
\hline 6 Pinch strength $(\mathrm{kg})$ & 1 & & Mean Difference (IV, Fixed, 95\% CI) & Totals not selected \\
\hline $\begin{array}{l}\text { 6.1 Pinch strength at } 3 \\
\text { months }\end{array}$ & 1 & & Mean Difference (IV, Fixed, 95\% CI) & $0.0[0.0,0.0]$ \\
\hline $\begin{array}{l}\text { 6.2 Pinch strength at } 6 \\
\text { months }\end{array}$ & 1 & & Mean Difference (IV, Fixed, 95\% CI) & $0.0[0.0,0.0]$ \\
\hline 7 Range of motion at 3 months & 1 & & Mean Difference (IV, Fixed, 95\% CI) & Totals not selected \\
\hline $\begin{array}{l}\text { 7.1 Extension-flexion arc } \\
\text { (degrees) }\end{array}$ & 1 & & Mean Difference (IV, Fixed, 95\% CI) & $0.0[0.0,0.0]$ \\
\hline 7.2 Pronation (degrees) & 1 & & Mean Difference (IV, Fixed, 95\% CI) & $0.0[0.0,0.0]$ \\
\hline 7.3 Supination (degrees) & 1 & & Mean Difference (IV, Fixed, 95\% CI) & $0.0[0.0,0.0]$ \\
\hline 7.4 Flexion (degrees) & 1 & & Mean Difference (IV, Fixed, 95\% CI) & $0.0[0.0,0.0]$ \\
\hline 7.5 Extension (degrees) & 1 & & Mean Difference (IV, Fixed, 95\% CI) & $0.0[0.0,0.0]$ \\
\hline 7.6 Radial deviation (degrees) & 1 & & Mean Difference (IV, Fixed, 95\% CI) & $0.0[0.0,0.0]$ \\
\hline 7.7 Ulnar deviation (degrees) & 1 & & Mean Difference (IV, Fixed, 95\% CI) & $0.0[0.0,0.0]$ \\
\hline 8 Range of motion at 6 months & 1 & & Mean Difference (IV, Fixed, 95\% CI) & Totals not selected \\
\hline $\begin{array}{l}\text { 8.1 Extension-flexion arc } \\
\text { (degrees) }\end{array}$ & 1 & & Mean Difference (IV, Fixed, 95\% CI) & $0.0[0.0,0.0]$ \\
\hline 8.2 Pronation (degrees) & 1 & & Mean Difference (IV, Fixed, 95\% CI) & $0.0[0.0,0.0]$ \\
\hline 8.3 Supination (degrees) & 1 & & Mean Difference (IV, Fixed, 95\% CI) & $0.0[0.0,0.0]$ \\
\hline 8.4 Flexion (degrees) & 1 & & Mean Difference (IV, Fixed, 95\% CI) & $0.0[0.0,0.0]$ \\
\hline
\end{tabular}

Rehabilitation for distal radial fractures in adults (Review)

Copyright $\odot 2015$ The Cochrane Collaboration. Published by John Wiley \& Sons, Ltd. 
8.5 Extension (degrees)

8.6 Radial deviation (degrees)

8.7 Ulnar deviation (degrees)

9 Complications

9.1 Carpal tunnel release 2-3 months post-initial treatment

9.2 Loss of alignment of lunar facet fragment

9.3 Extensor pollicis longus

tendon rupture

9.4 Implant removal for

tendon irritation
Mean Difference (IV, Fixed, 95\% CI)

Mean Difference (IV, Fixed, 95\% CI)

Mean Difference (IV, Fixed, 95\% CI)

Risk Ratio (M-H, Fixed, 95\% CI)

Risk Ratio (M-H, Fixed, 95\% CI)

Risk Ratio (M-H, Fixed, 95\% CI)

Risk Ratio (M-H, Fixed, 95\% CI)

Risk Ratio (M-H, Fixed, 95\% CI)
$0.0[0.0,0.0]$

$0.0[0.0,0.0]$

$0.0[0.0,0.0]$

Totals not selected

$0.0[0.0,0.0]$

$0.0[0.0,0.0]$

$0.0[0.0,0.0]$

$0.0[0.0,0.0]$

\section{Comparison 21. Accelerated (start 2 weeks) versus usual (start 6 weeks) rehabilitation post-surgery}

\begin{tabular}{|c|c|c|c|c|}
\hline Outcome or subgroup title & $\begin{array}{l}\text { No. of } \\
\text { studies }\end{array}$ & $\begin{array}{c}\text { No. of } \\
\text { participants }\end{array}$ & Statistical method & Effect size \\
\hline $\begin{array}{l}1 \text { DASH score (0 to } 100 \text { : higher } \\
\text { scores = worse upper-extremity } \\
\text { function) }\end{array}$ & 1 & & Mean Difference (IV, Fixed, 95\% CI) & Totals not selected \\
\hline 1.1 At 8 weeks & 1 & & Mean Difference (IV, Fixed, 95\% CI) & $0.0[0.0,0.0]$ \\
\hline 1.2 At 12 weeks & 1 & & Mean Difference (IV, Fixed, 95\% CI) & $0.0[0.0,0.0]$ \\
\hline 1.3 At 6 months & 1 & & Mean Difference (IV, Fixed, 95\% CI) & $0.0[0.0,0.0]$ \\
\hline 2 Grip strength (lb) & 1 & & Mean Difference (IV, Fixed, 95\% CI) & Totals not selected \\
\hline 2.1 Grip strength at 12 weeks & 1 & & Mean Difference (IV, Fixed, 95\% CI) & $0.0[0.0,0.0]$ \\
\hline 2.2 Grip strength at 6 months & 1 & & Mean Difference (IV, Fixed, 95\% CI) & $0.0[0.0,0.0]$ \\
\hline 3 Pinch strength (lb) & 1 & & Mean Difference (IV, Fixed, 95\% CI) & Totals not selected \\
\hline 3.1 Pinch strength at 12 weeks & 1 & & Mean Difference (IV, Fixed, 95\% CI) & $0.0[0.0,0.0]$ \\
\hline $\begin{array}{l}3.2 \text { Pinch strength at } 6 \\
\text { months }\end{array}$ & 1 & & Mean Difference (IV, Fixed, 95\% CI) & $0.0[0.0,0.0]$ \\
\hline 4 Range of motion at 12 weeks & 1 & & Mean Difference (IV, Fixed, 95\% CI) & Totals not selected \\
\hline 4.1 Pronation (degrees) & 1 & & Mean Difference (IV, Fixed, 95\% CI) & $0.0[0.0,0.0]$ \\
\hline 4.2 Supination (degrees) & 1 & & Mean Difference (IV, Fixed, 95\% CI) & $0.0[0.0,0.0]$ \\
\hline 4.3 Flexion (degrees) & 1 & & Mean Difference (IV, Fixed, 95\% CI) & $0.0[0.0,0.0]$ \\
\hline 4.4 Extension (degrees) & 1 & & Mean Difference (IV, Fixed, 95\% CI) & $0.0[0.0,0.0]$ \\
\hline 5 Range of motion at 6 months & 1 & & Mean Difference (IV, Fixed, 95\% CI) & Totals not selected \\
\hline 5.1 Pronation (degrees) & 1 & & Mean Difference (IV, Fixed, 95\% CI) & $0.0[0.0,0.0]$ \\
\hline 5.2 Supination (degrees) & 1 & & Mean Difference (IV, Fixed, 95\% CI) & $0.0[0.0,0.0]$ \\
\hline 5.3 Flexion (degrees) & 1 & & Mean Difference (IV, Fixed, 95\% CI) & $0.0[0.0,0.0]$ \\
\hline 5.4 Extension (degrees) & 1 & & Mean Difference (IV, Fixed, 95\% CI) & $0.0[0.0,0.0]$ \\
\hline 6 Complications & 1 & & Risk Ratio (M-H, Fixed, 95\% CI) & Totals not selected \\
\hline 6.1 Carpal tunnel syndrome & 1 & & Risk Ratio (M-H, Fixed, 95\% CI) & $0.0[0.0,0.0]$ \\
\hline $\begin{array}{l}\text { 6.2 Loss of alignment or } \\
\text { non-union }\end{array}$ & 1 & & Risk Ratio (M-H, Fixed, 95\% CI) & $0.0[0.0,0.0]$ \\
\hline $\begin{array}{l}6.3 \text { Extensor pollicis longus } \\
\text { tendon rupture }\end{array}$ & 1 & & Risk Ratio (M-H, Fixed, 95\% CI) & $0.0[0.0,0.0]$ \\
\hline $\begin{array}{l}6.4 \text { Re-operation to remove } \\
\text { screw }\end{array}$ & 1 & & Risk Ratio (M-H, Fixed, 95\% CI) & $0.0[0.0,0.0]$ \\
\hline
\end{tabular}

Rehabilitation for distal radial fractures in adults (Review)

Copyright $\odot 2015$ The Cochrane Collaboration. Published by John Wiley \& Sons, Ltd. 
Analysis I.I. Comparison I Early (during immobilisation) occupational or hand therapy versus no intervention (control), Outcome I Meeting criteria for attendance of post-immobilisation hand therapy group.

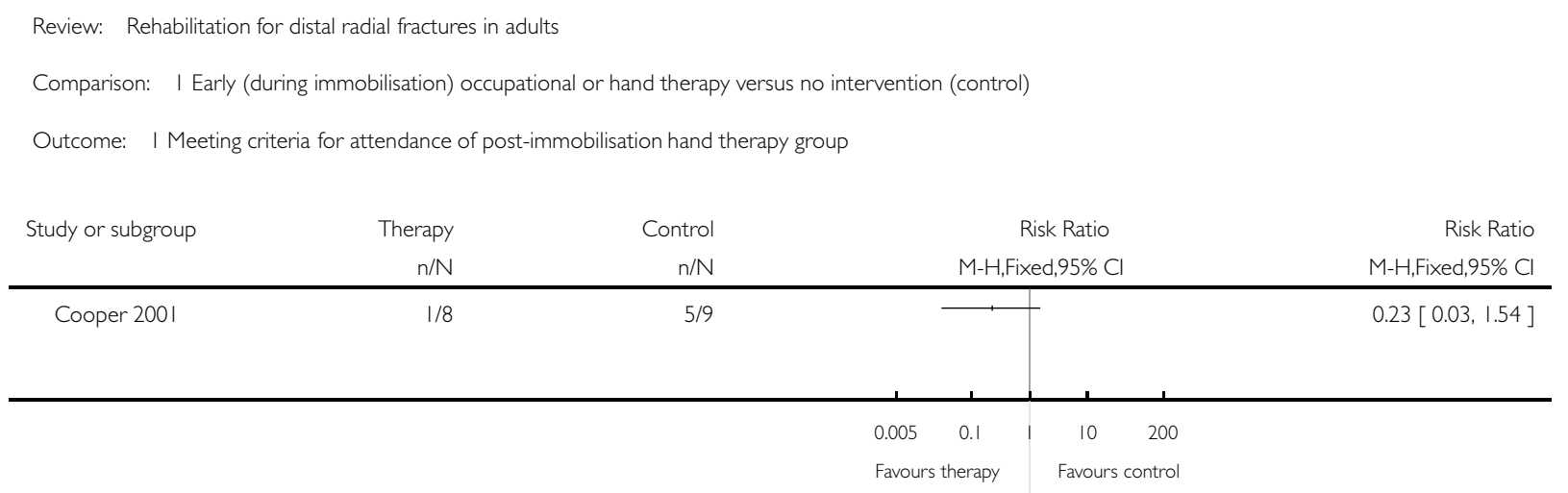

Analysis I.2. Comparison I Early (during immobilisation) occupational or hand therapy versus no intervention (control), Outcome 2 Grip strength $(\mathrm{kg})$ at 4 weeks (post-immobilisation).

Review: Rehabilitation for distal radial fractures in adults

Comparison: I Early (during immobilisation) occupational or hand therapy versus no intervention (control)

Outcome: 2 Grip strength $(\mathrm{kg})$ at 4 weeks (post-immobilisation)

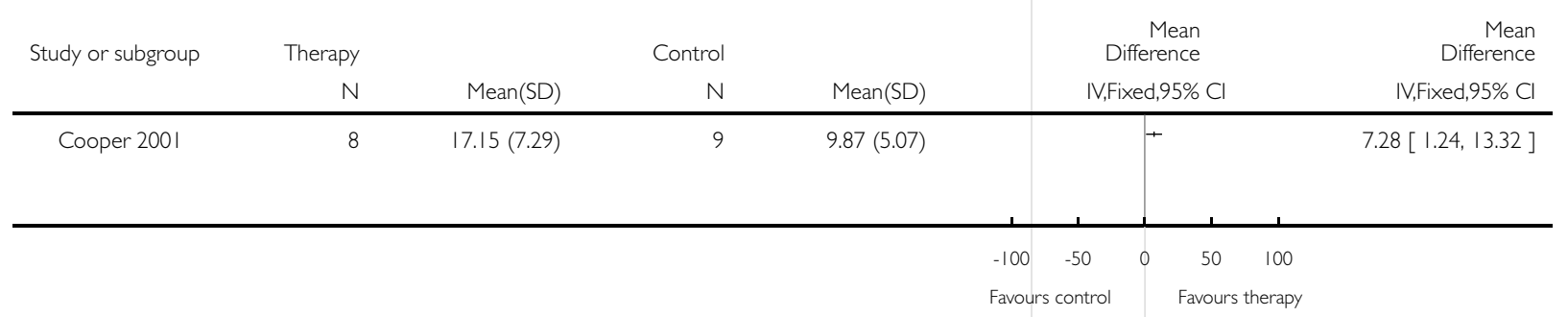


Analysis I.3. Comparison I Early (during immobilisation) occupational or hand therapy versus no intervention (control), Outcome 3 Range of motion at 4 weeks (post-immobilisation).

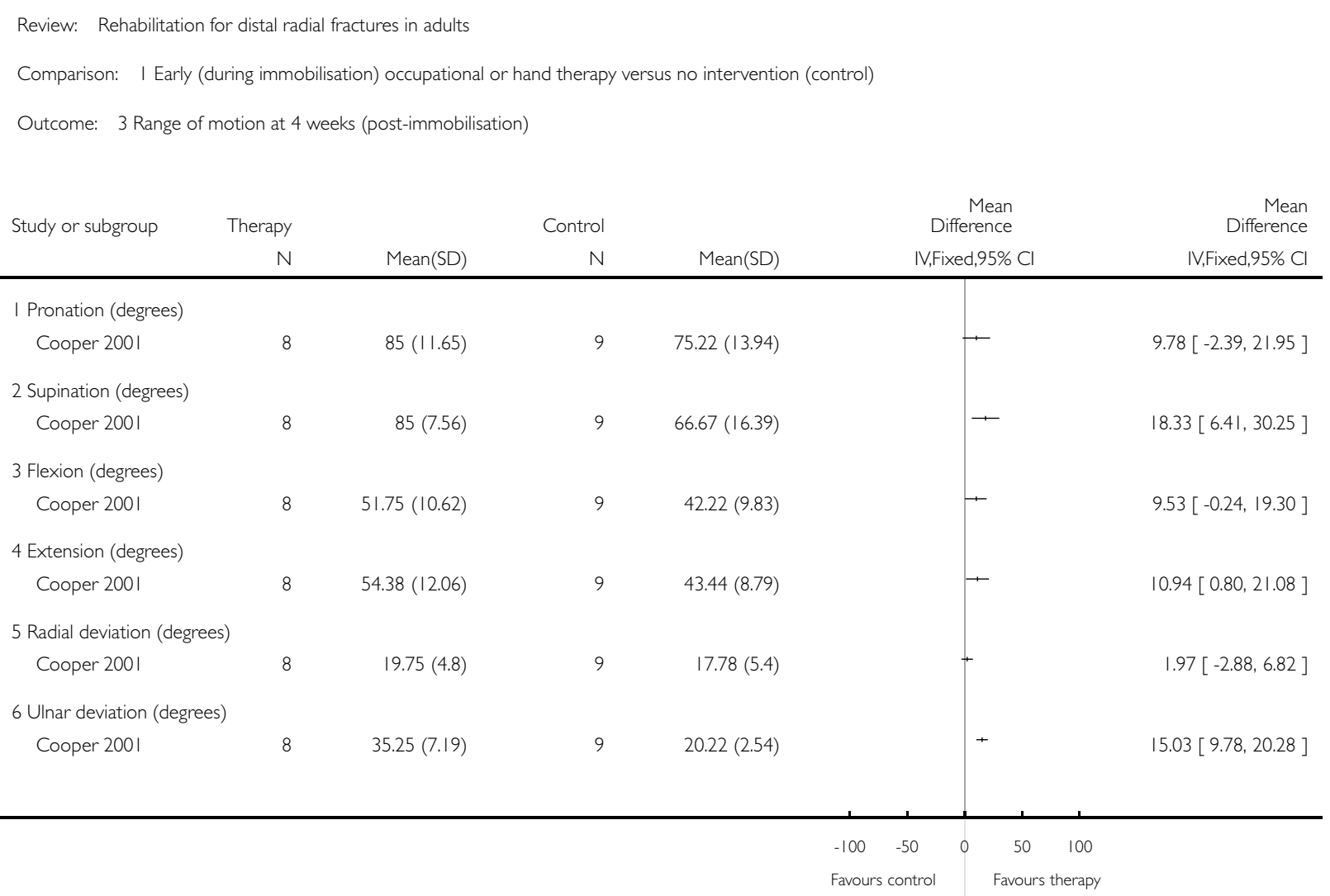


Analysis 1.4. Comparison I Early (during immobilisation) occupational or hand therapy versus no intervention (control), Outcome 4 Oedema $(\mathrm{mL})$ at 4 weeks (post-immobilisation).

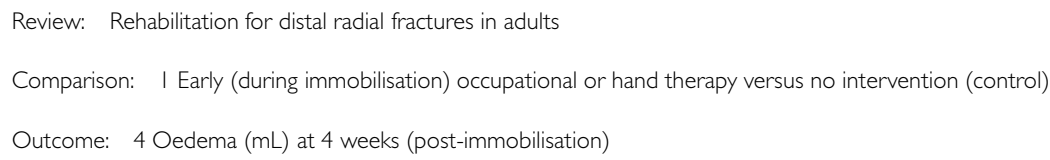

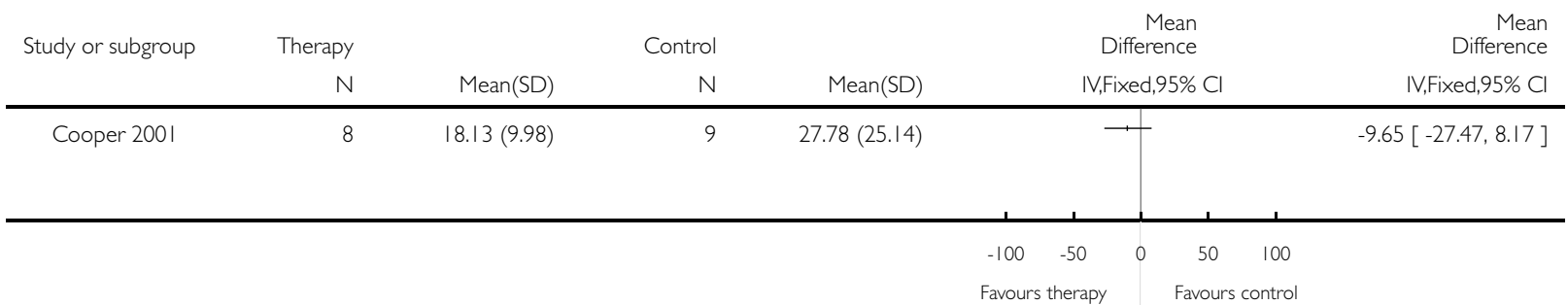

Analysis I.5. Comparison I Early (during immobilisation) occupational or hand therapy versus no intervention (control), Outcome 5 Any pain at rest at 4 weeks (post-immobilisation).

Review: Rehabilitation for distal radial fractures in adults

Comparison: | Early (during immobilisation) occupational or hand therapy versus no intervention (control)

Outcome: 5 Any pain at rest at 4 weeks (post-immobilisation)

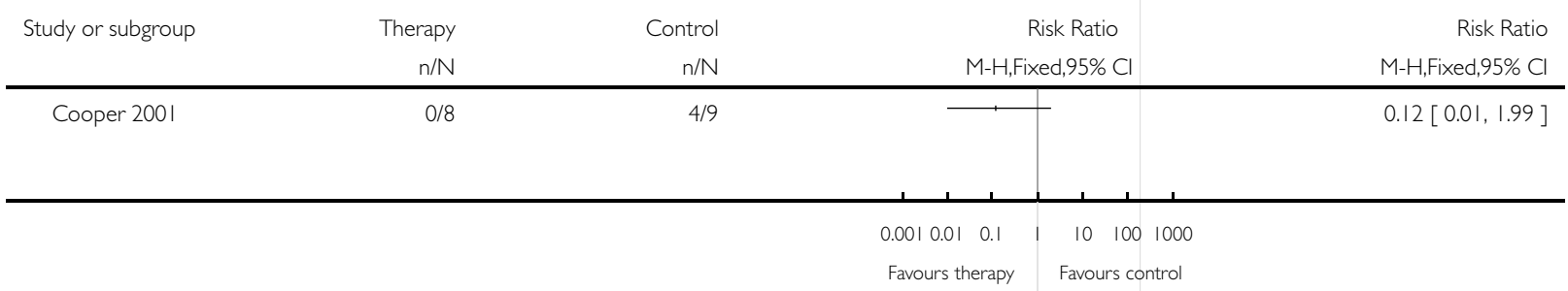


Analysis I.6. Comparison I Early (during immobilisation) occupational or hand therapy versus no intervention (control), Outcome 6 Finger mobility at 4 weeks (post-immobilisation).

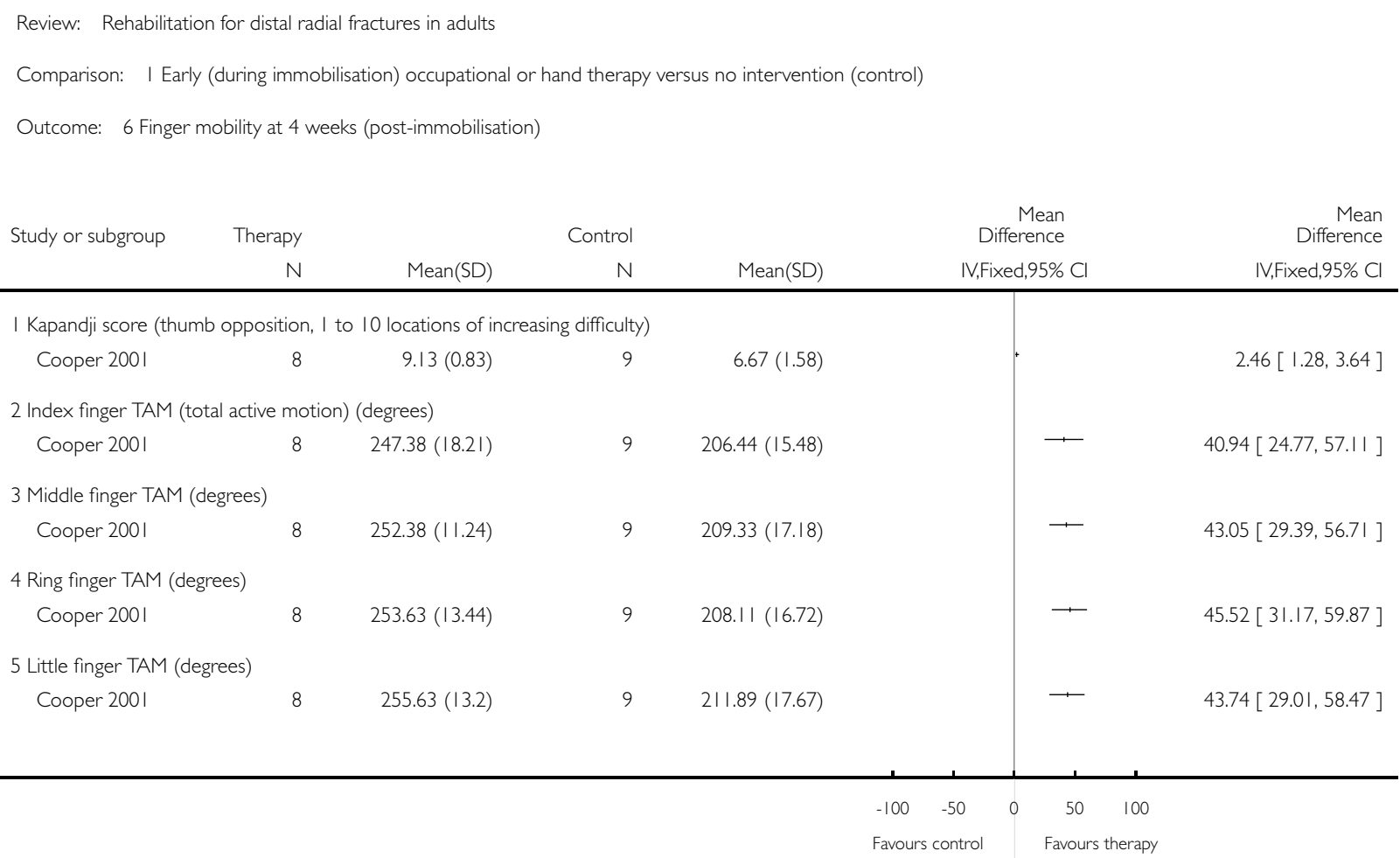


Analysis I.7. Comparison I Early (during immobilisation) occupational or hand therapy versus no intervention (control), Outcome 7 Complications.

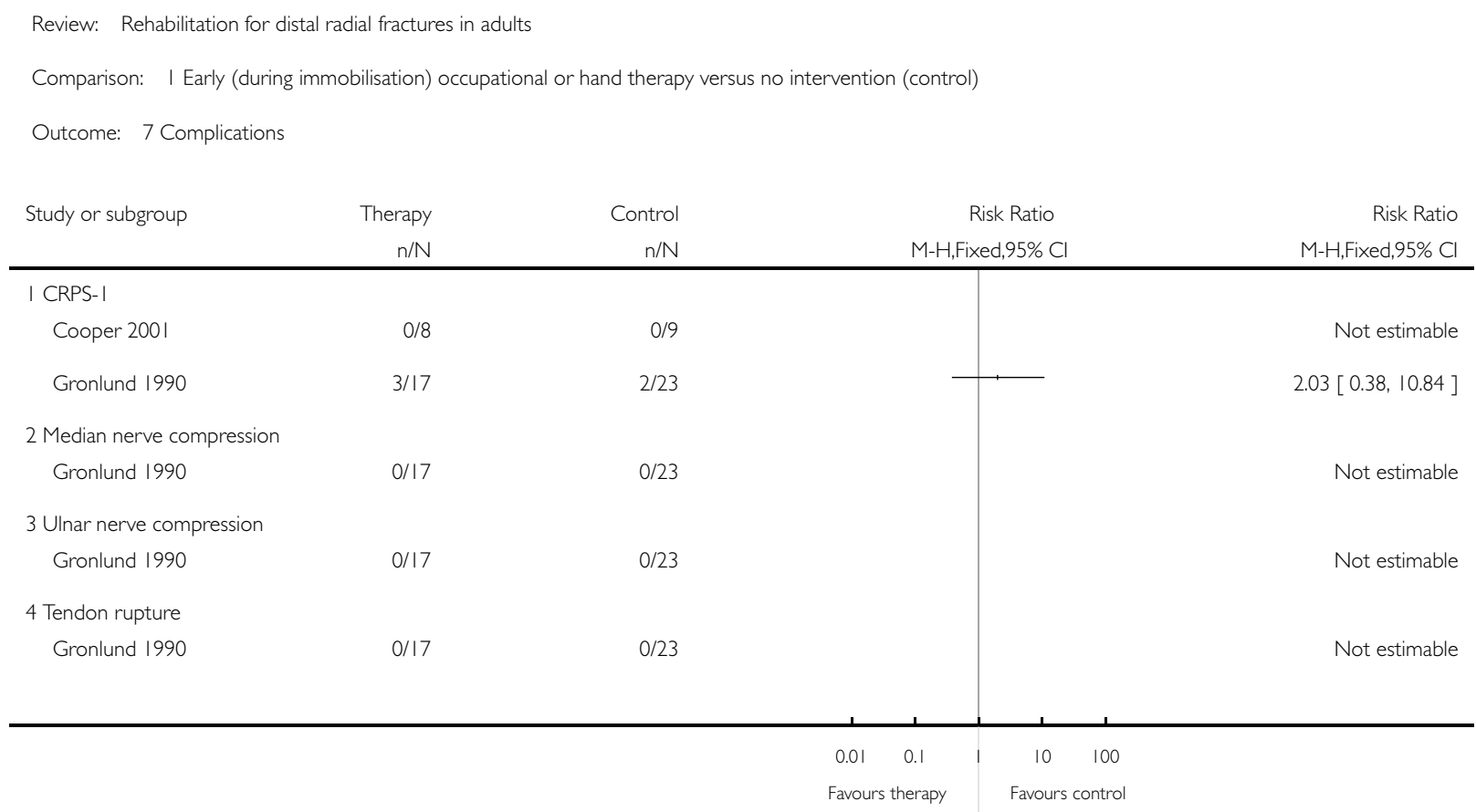


Analysis 2.1. Comparison 2 Cyclic pneumatic soft tissue compression during immobilisation versus no intervention (control), Outcome I Grip strength (kg).

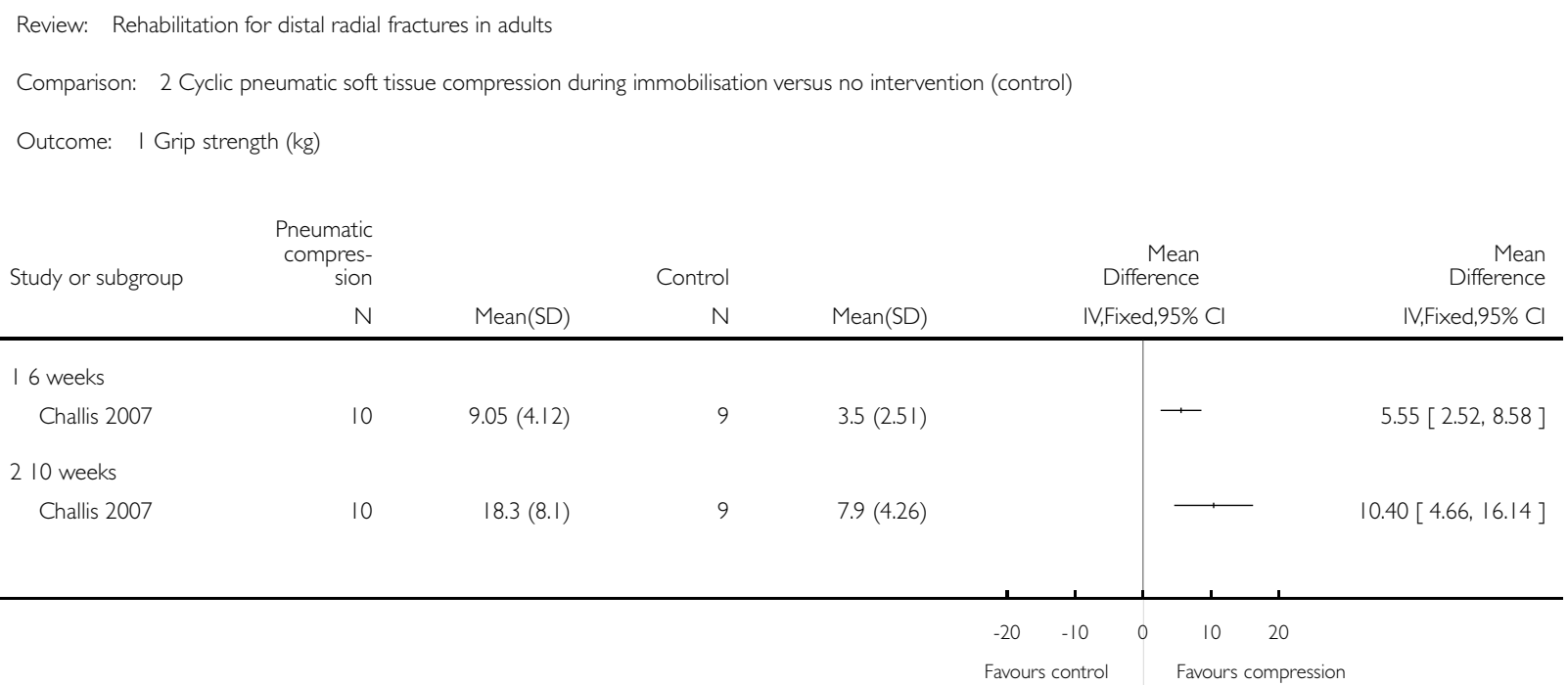

Analysis 2.2. Comparison 2 Cyclic pneumatic soft tissue compression during immobilisation versus no intervention (control), Outcome 2 Pinch strength (kg).

Review: Rehabilitation for distal radial fractures in adults

Comparison: 2 Cyclic pneumatic soft tissue compression during immobilisation versus no intervention (control)

Outcome: 2 Pinch strength $(\mathrm{kg})$

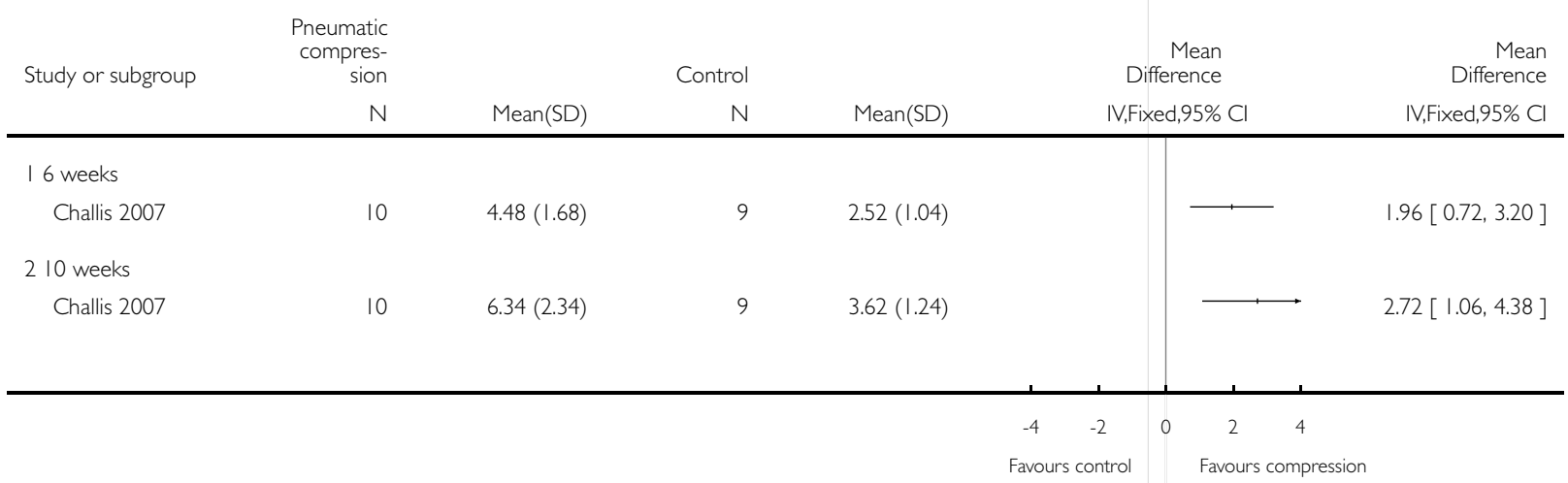


Analysis 2.3. Comparison 2 Cyclic pneumatic soft tissue compression during immobilisation versus no intervention (control), Outcome 3 Range of motion (degrees).

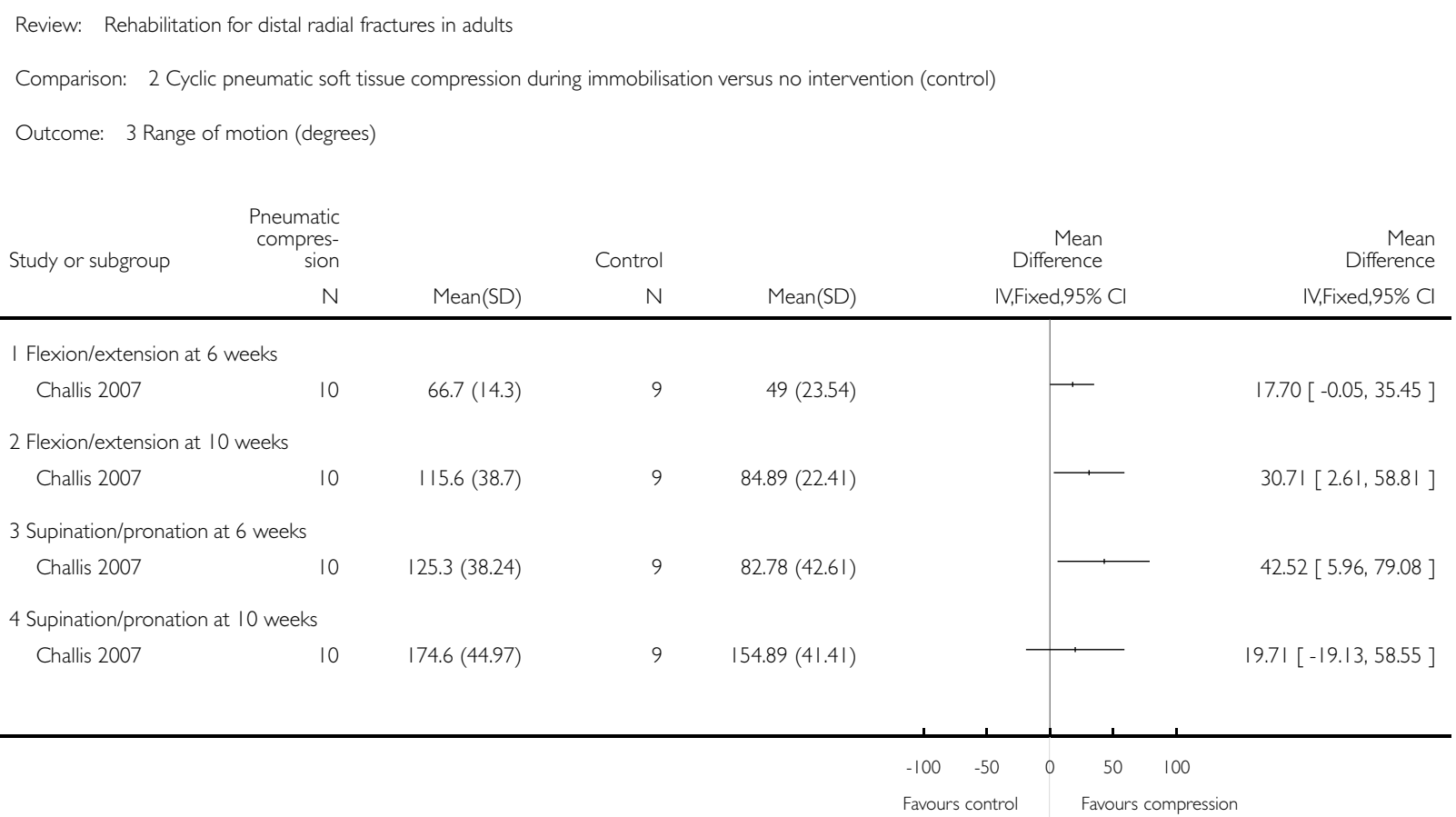


Analysis 3.I. Comparison 3 Early (during external fixation) digit mobilisation programme versus no intervention (control), Outcome I Manual Ability Measure-36 - Taiwan version 45 questions (36 to I80: best result).

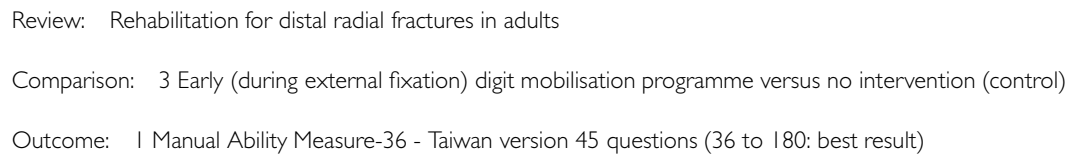

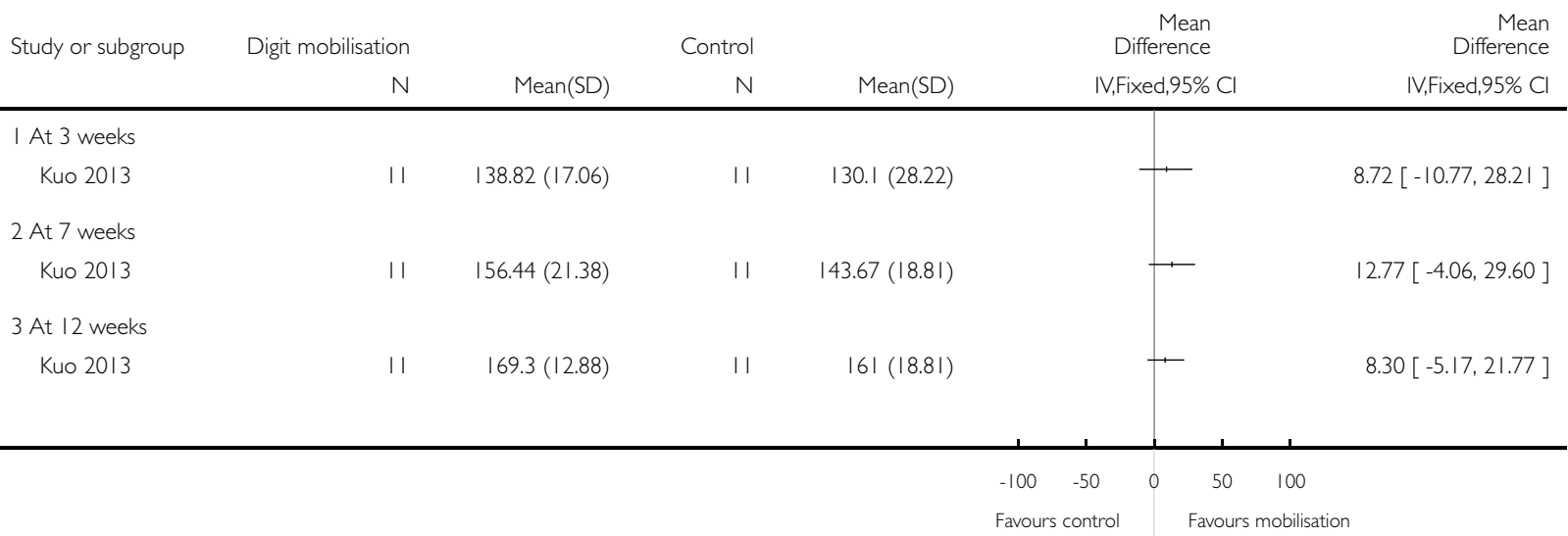


Analysis 3.2. Comparison 3 Early (during external fixation) digit mobilisation programme versus no intervention (control), Outcome 2 Grip, pinch and 'three jaw chuck' pinch strengths (\% of uninvolved hand).

Review: Rehabilitation for distal radial fractures in adults

Comparison: 3 Early (during external fixation) digit mobilisation programme versus no intervention (control)

Outcome: 2 Grip, pinch and 'three jaw chuck' pinch strengths (\% of uninvolved hand)

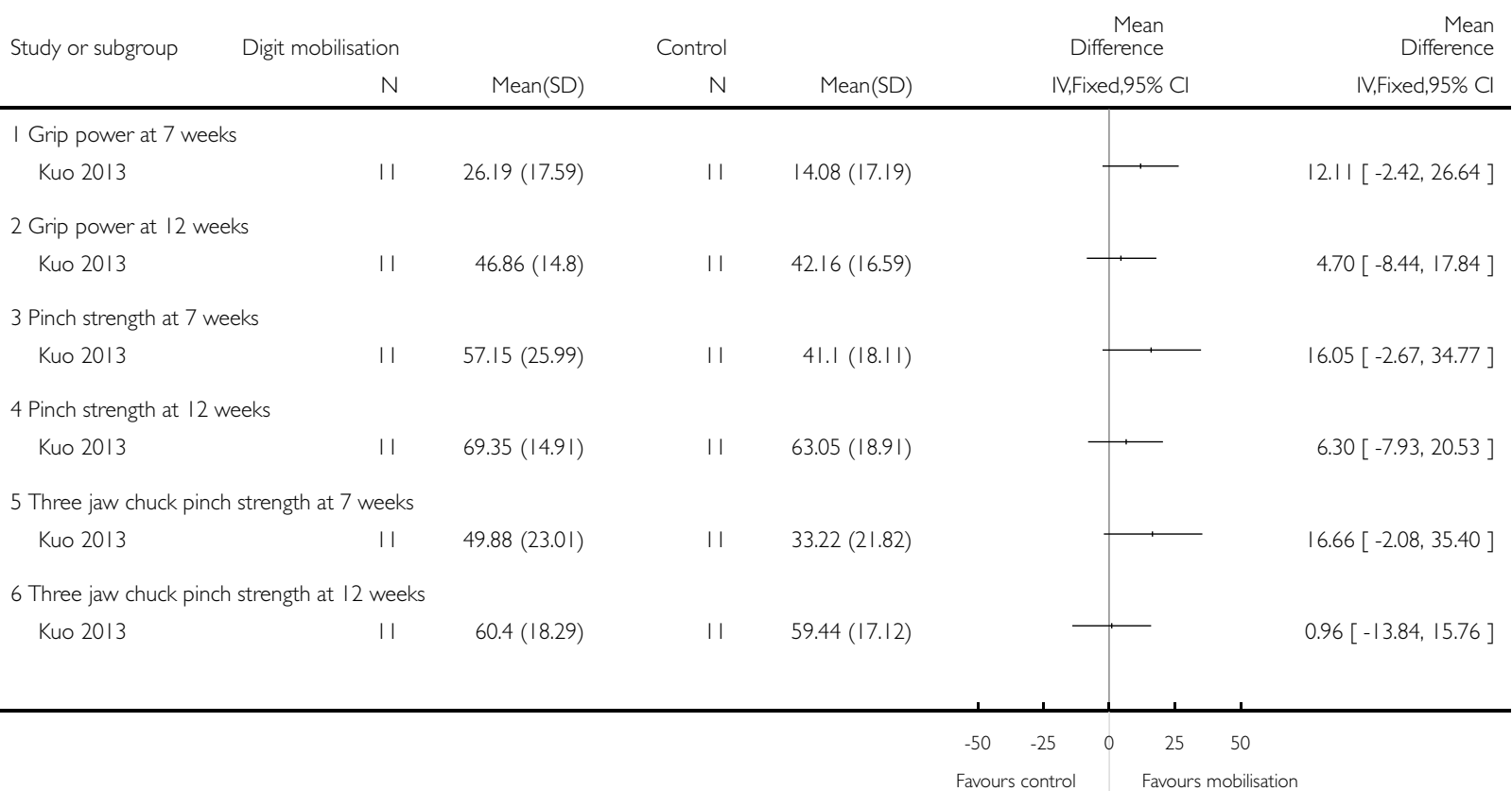


Analysis 3.3. Comparison 3 Early (during external fixation) digit mobilisation programme versus no intervention (control), Outcome 3 Range of motion (\% of other hand).

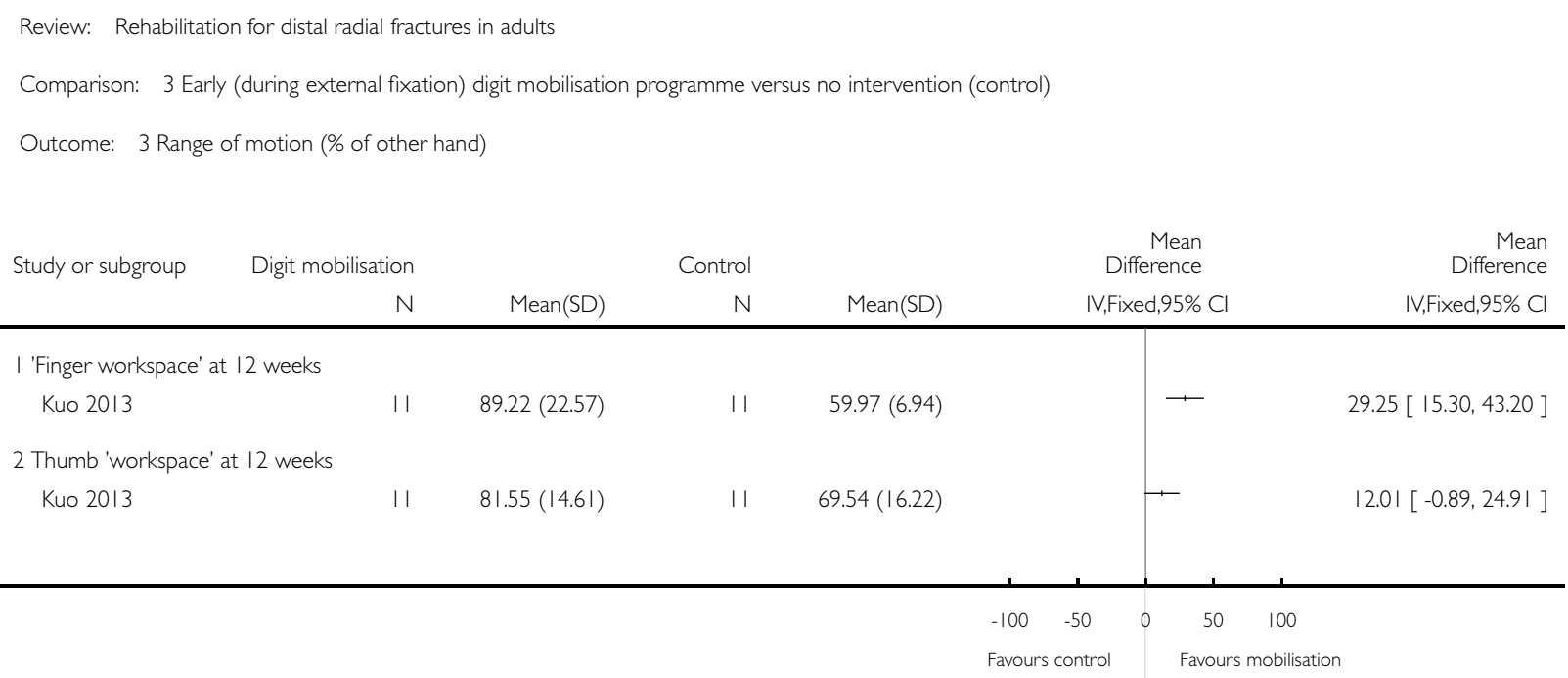

Analysis 4.I. Comparison 4 Pulsed electromagnetic field (PEMF) (during cast immobilisation) versus no intervention (control), Outcome I PRWE scores at 2 to 3 days after cast removal.

Review: Rehabilitation for distal radial fractures in adults

Comparison: 4 Pulsed electromagnetic field (PEMF) (during cast immobilisation) versus no intervention (control)

Outcome: I PRWE scores at 2 to 3 days after cast removal

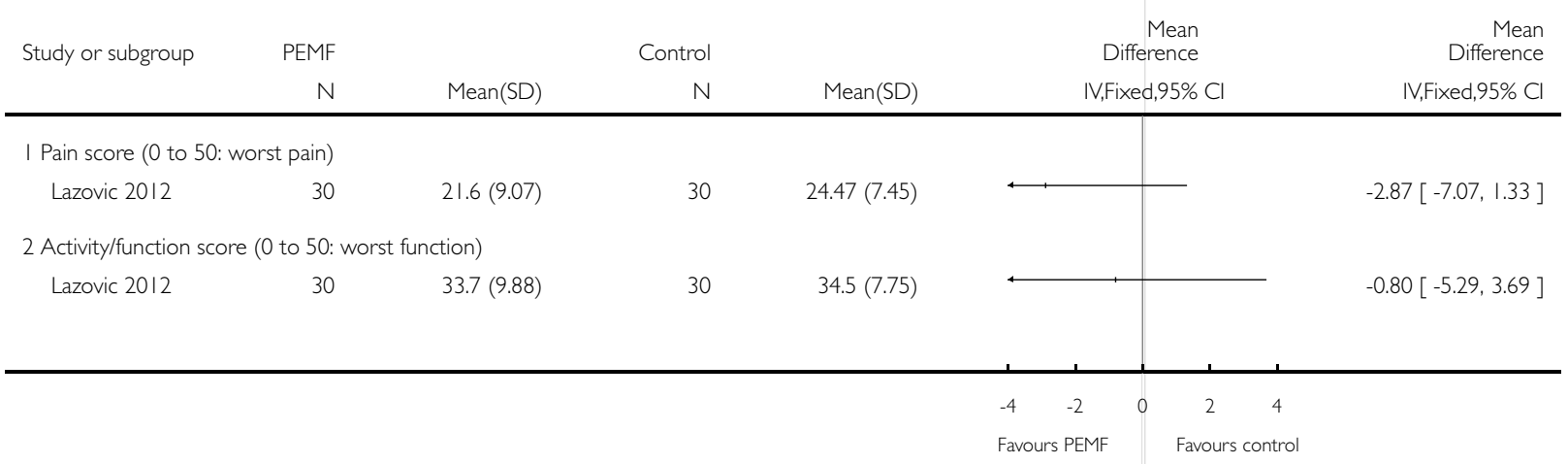


Analysis 4.2. Comparison 4 Pulsed electromagnetic field (PEMF) (during cast immobilisation) versus no intervention (control), Outcome 2 Range of motion at day 2 to 3 after cast removal.

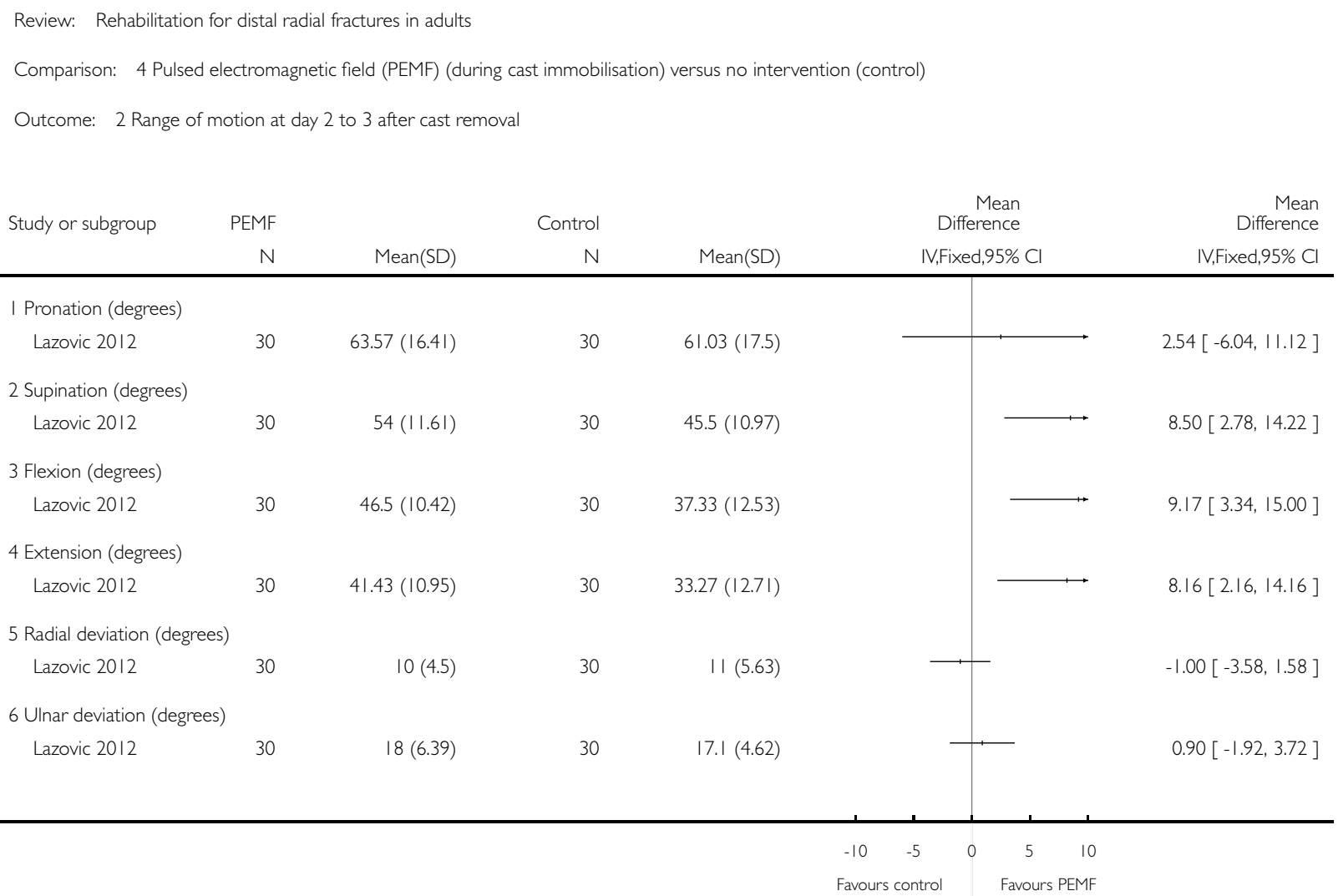


Analysis 4.3. Comparison 4 Pulsed electromagnetic field (PEMF) (during cast immobilisation) versus no intervention (control), Outcome 3 Hand oedema: difference between hands in circumference $(\mathrm{mm})$.

Review: Rehabilitation for distal radial fractures in adults

Comparison: 4 Pulsed electromagnetic field (PEMF) (during cast immobilisation) versus no intervention (control)

Outcome: 3 Hand oedema: difference between hands in circumference $(\mathrm{mm})$

\begin{tabular}{|c|c|c|c|c|c|c|c|c|c|}
\hline \multirow[t]{2}{*}{ Study or subgroup } & \multirow{2}{*}{$\begin{array}{r}\text { PEMF } \\
\mathrm{N}\end{array}$} & \multicolumn{3}{|c|}{ Control } & \multicolumn{4}{|c|}{$\begin{array}{r}\text { Mean } \\
\text { Difference }\end{array}$} & $\begin{array}{r}\text { Mean } \\
\text { Difference }\end{array}$ \\
\hline & & Mean(SD) & $\mathrm{N}$ & Mean(SD) & \multicolumn{4}{|c|}{ IV,Fixed,95\% Cl } & IV,Fixed,95\% Cl \\
\hline \multirow[t]{3}{*}{ Lazovic 2012} & 30 & $10.2(6.14)$ & 30 & | $8.17(7.44)$ & & 一 & & & $-7.97[-11.42,-4.52]$ \\
\hline & & & & & -20 & -10 & 10 & 20 & \\
\hline & & & & & Favo & S PEMF & Favour & ontrol & \\
\hline
\end{tabular}

Analysis 4.4. Comparison 4 Pulsed electromagnetic field (PEMF) (during cast immobilisation) versus no intervention (control), Outcome 4 Complications.

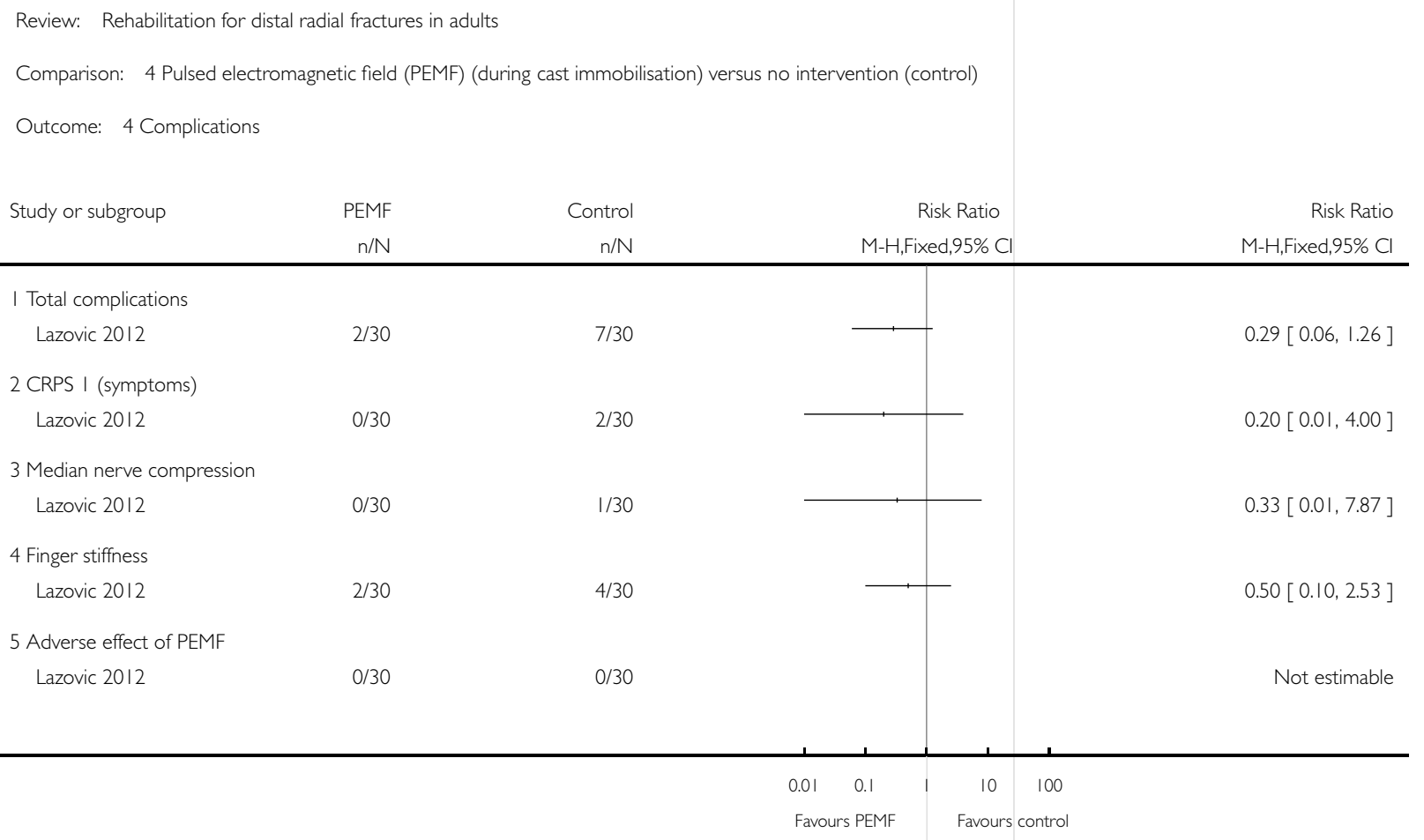

Rehabilitation for distal radial fractures in adults (Review)

Copyright $\odot 2015$ The Cochrane Collaboration. Published by John Wiley \& Sons, Ltd. 
Analysis 5.I. Comparison 5 Cross-education (strengthening exercises for opposite hand) versus no intervention (control), Outcome I Patient-Rated Wrist Evaluation (PRWE) (0 to I50: worst results).

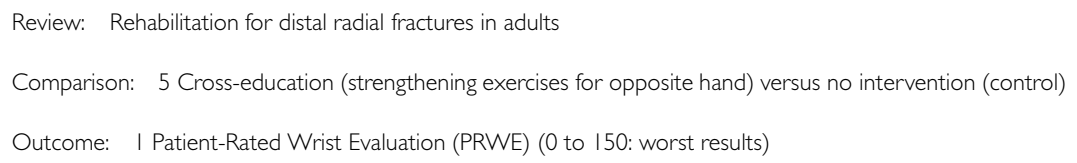

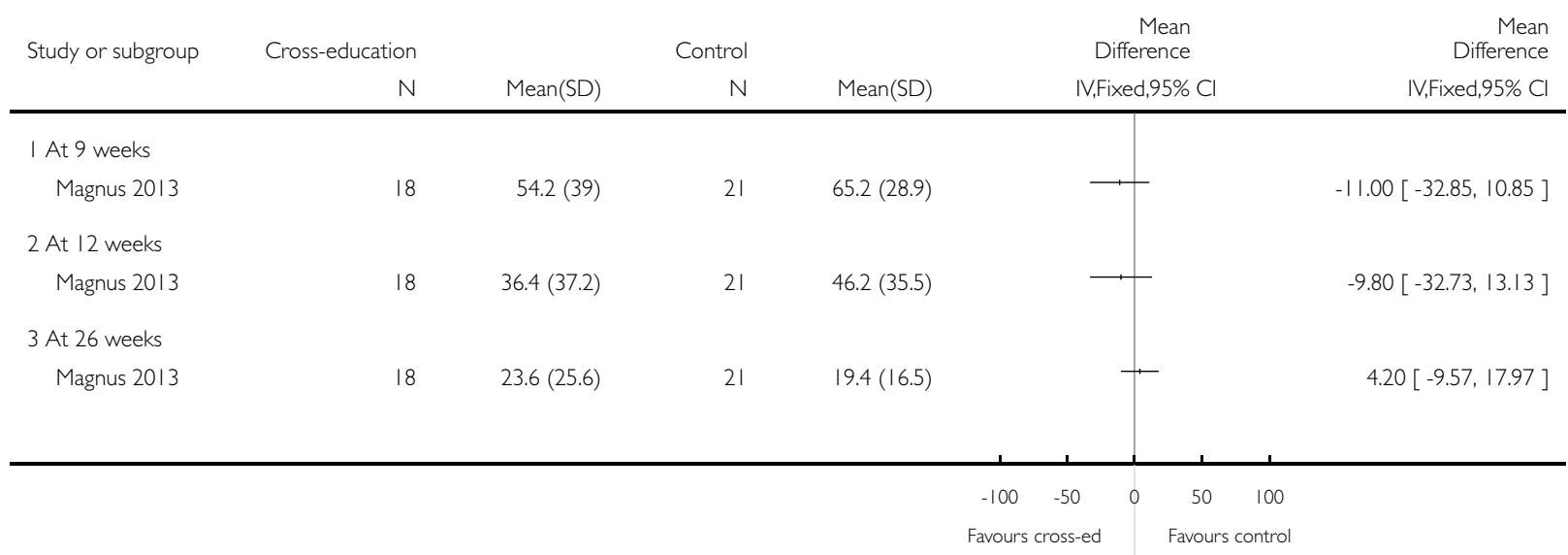


Analysis 5.2. Comparison 5 Cross-education (strengthening exercises for opposite hand) versus no intervention (control), Outcome 2 Grip strength of fractured hand (kg).

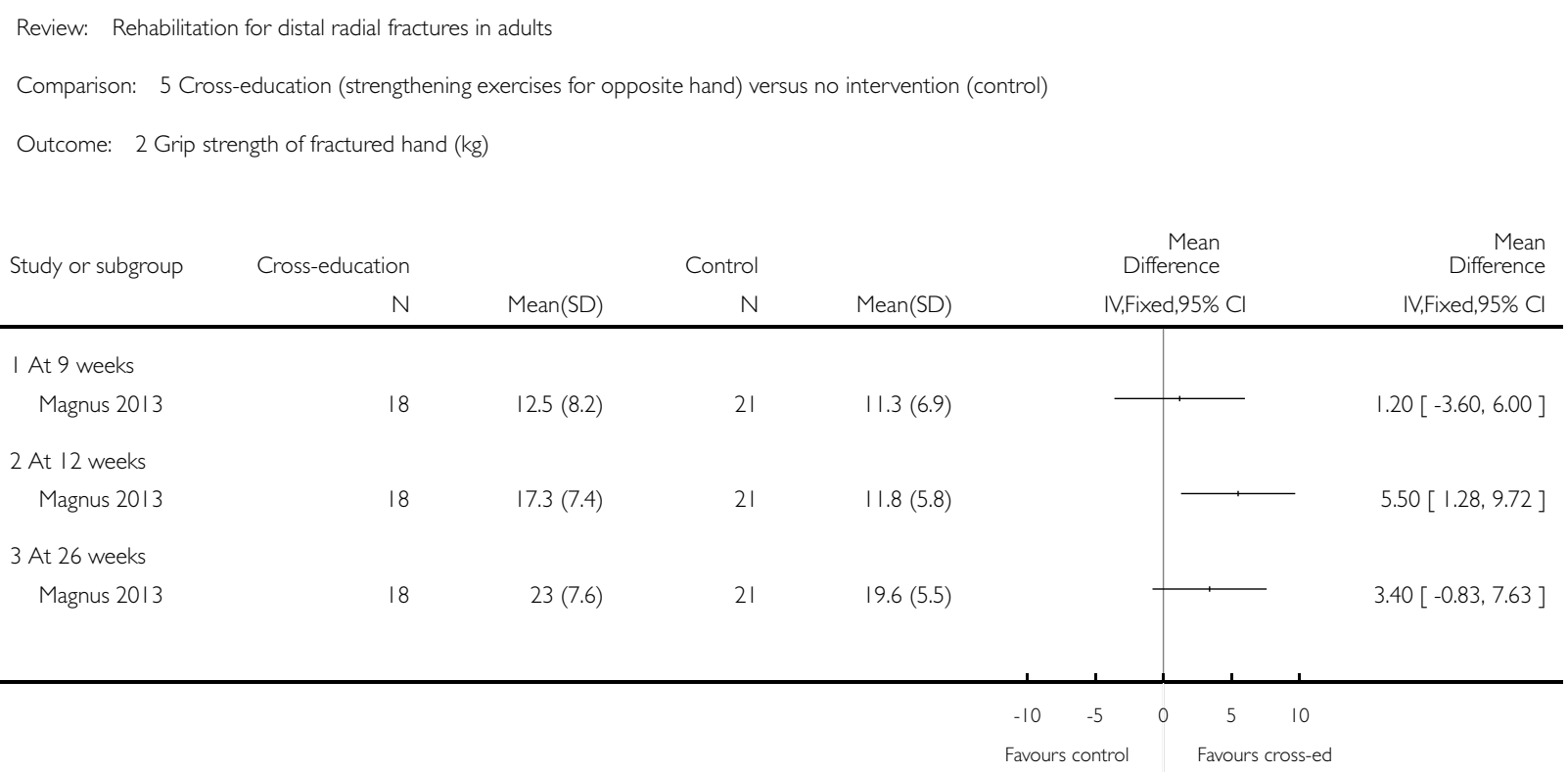


Analysis 5.3. Comparison 5 Cross-education (strengthening exercises for opposite hand) versus no intervention (control), Outcome 3 Range of motion: supination/pronation (degrees).

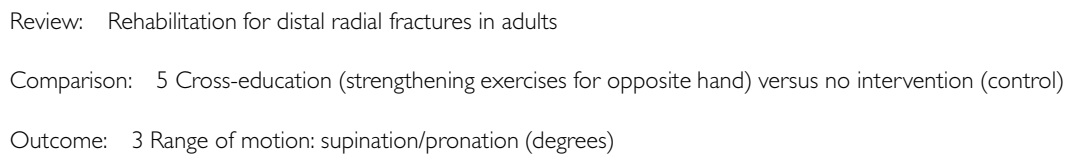

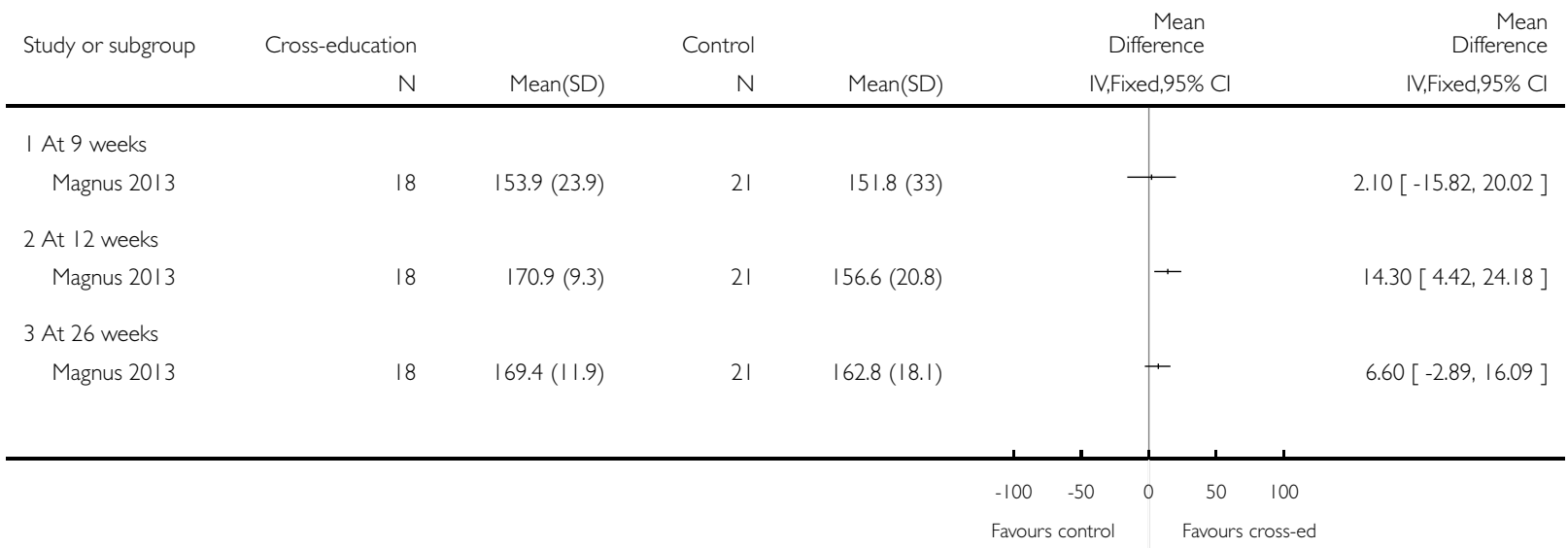


Analysis 5.4. Comparison 5 Cross-education (strengthening exercises for opposite hand) versus no intervention (control), Outcome 4 Range of motion: flexion/extension (degrees).

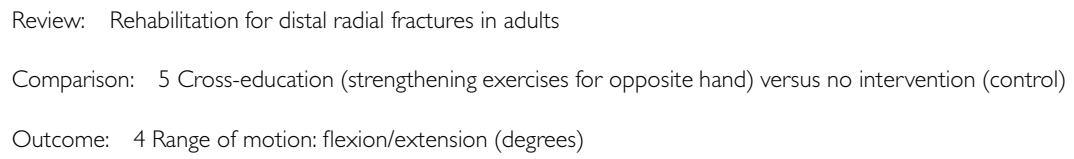

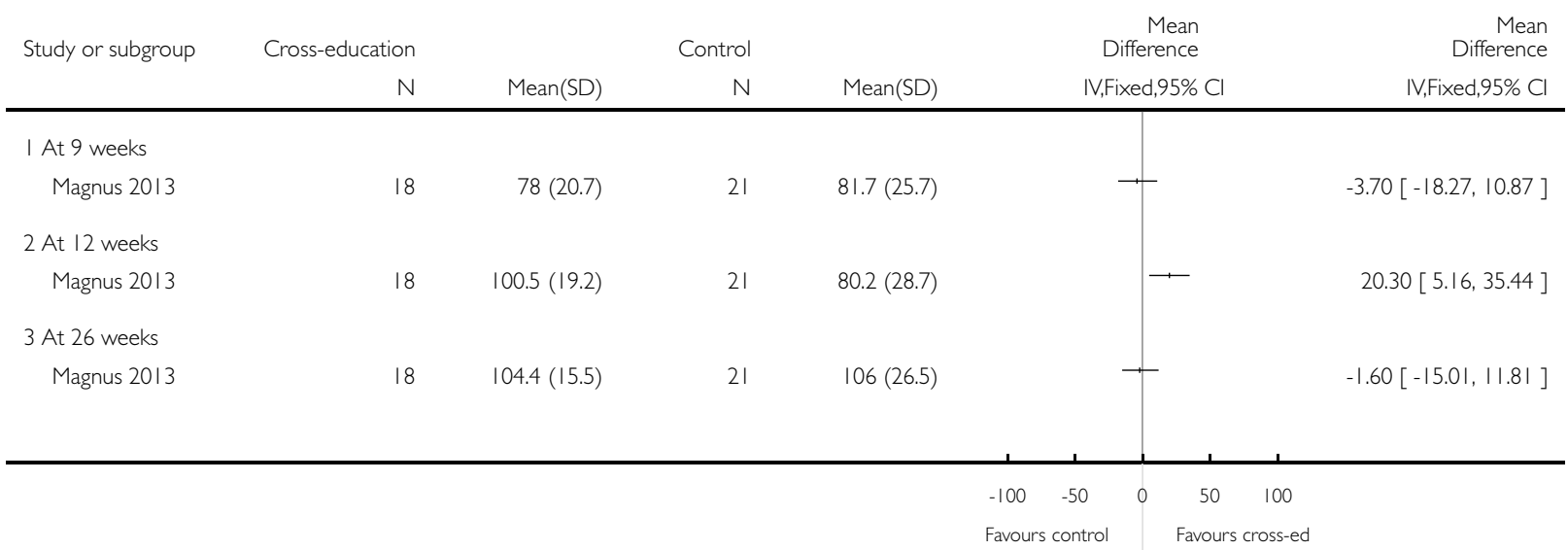


Analysis 6.I. Comparison 6 Physiotherapy (one session for home exercises) versus no intervention (control) (post-immobilisation), Outcome I PRWE scores at 3 and 6 weeks.

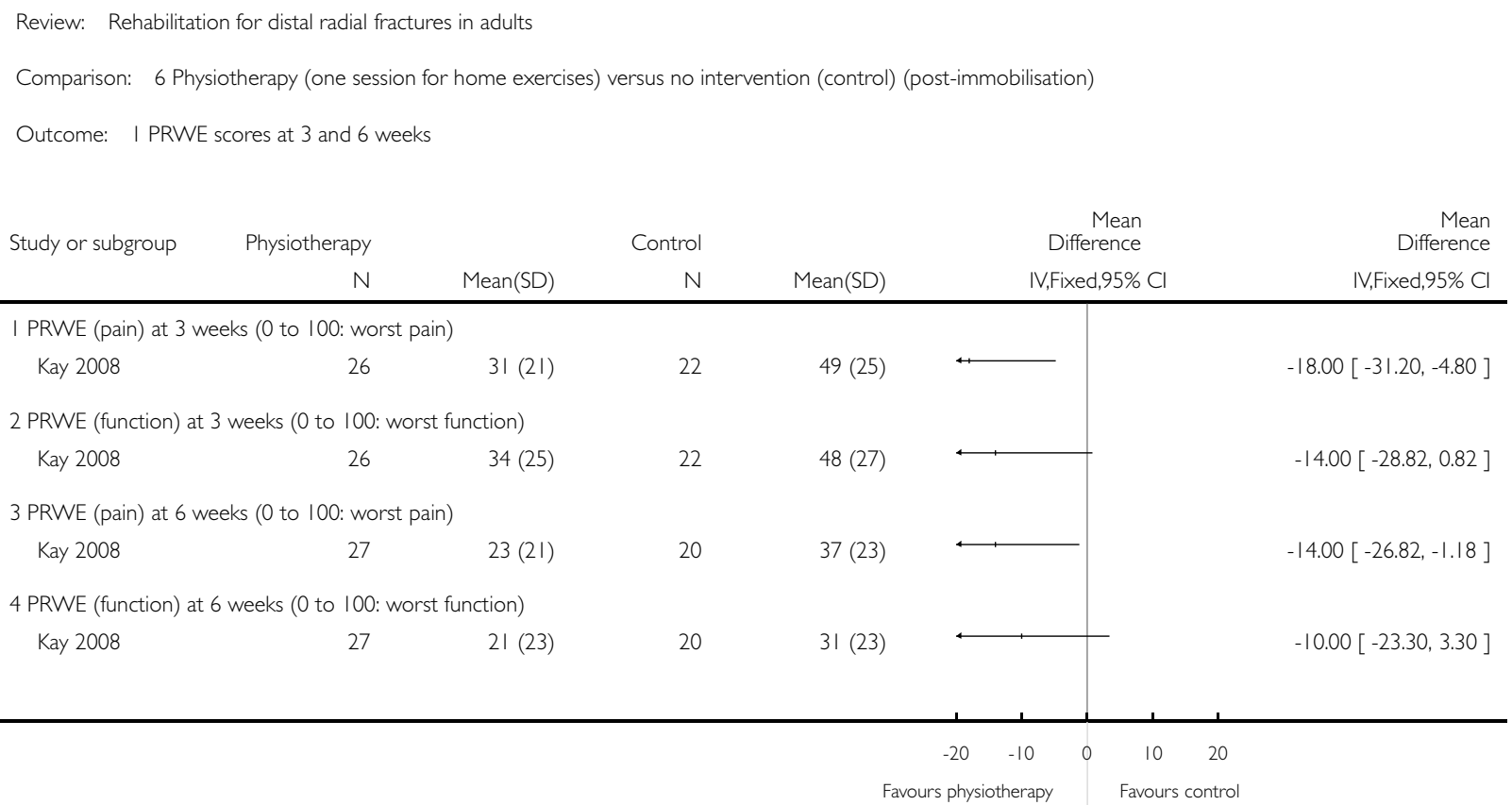


Analysis 6.2. Comparison 6 Physiotherapy (one session for home exercises) versus no intervention (control) (post-immobilisation), Outcome 2 QuickDASH scores at 3 and 6 weeks.

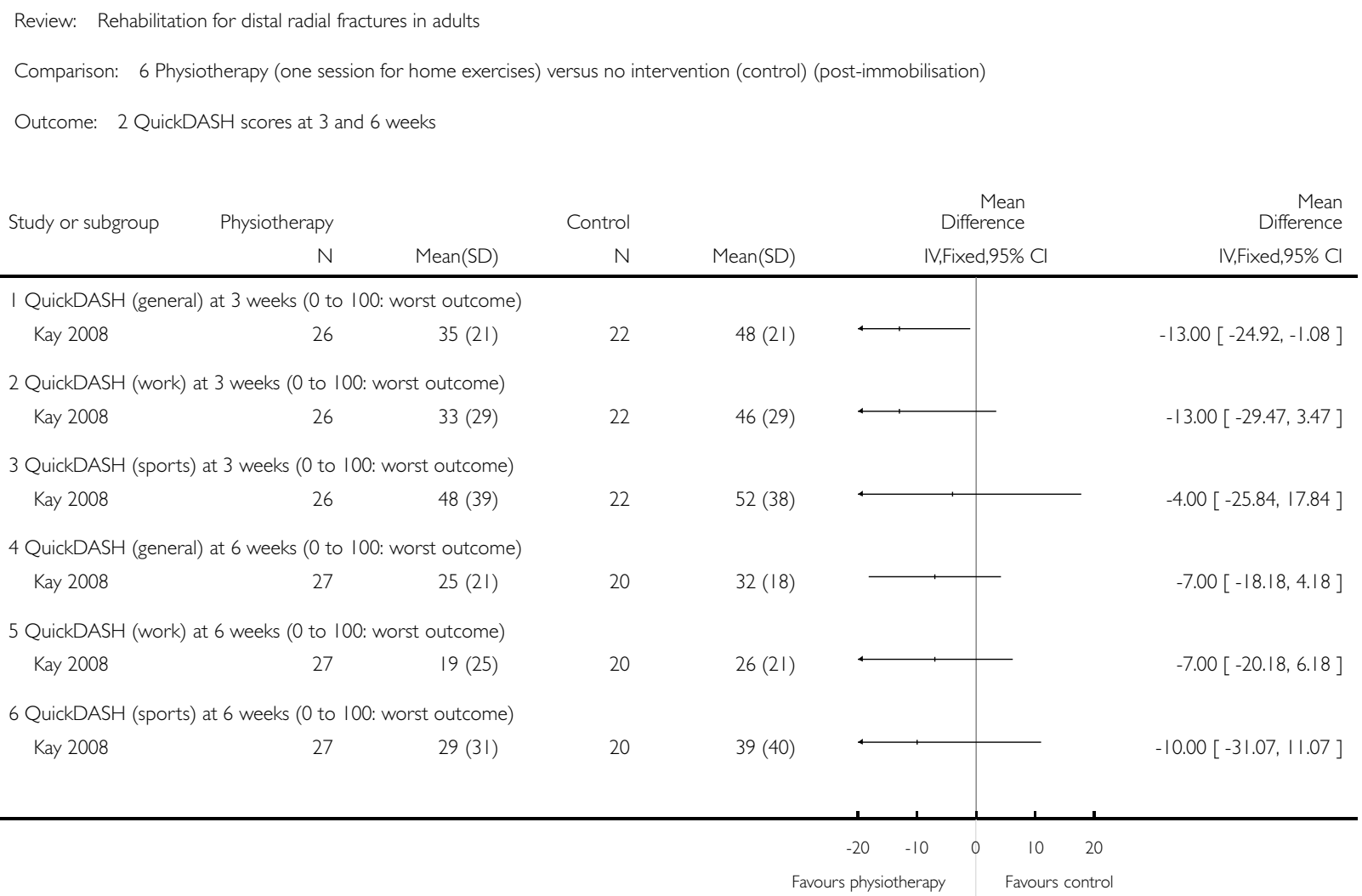


Analysis 6.3. Comparison 6 Physiotherapy (one session for home exercises) versus no intervention (control) (post-immobilisation), Outcome 3 Grip strength $(\mathrm{kg})$ at 3 and 6 weeks.

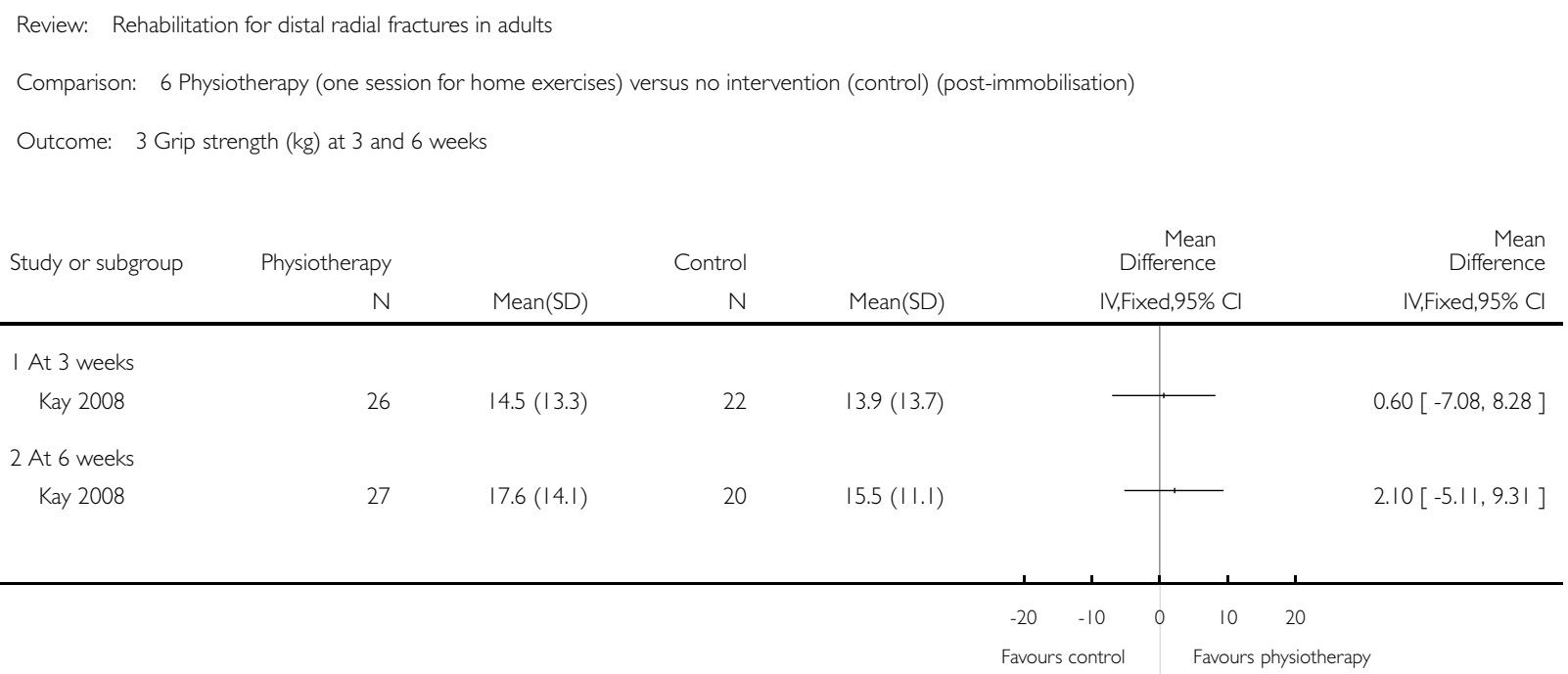


Analysis 6.4. Comparison 6 Physiotherapy (one session for home exercises) versus no intervention (control) (post-immobilisation), Outcome 4 Range of motion at 6 weeks (degrees).

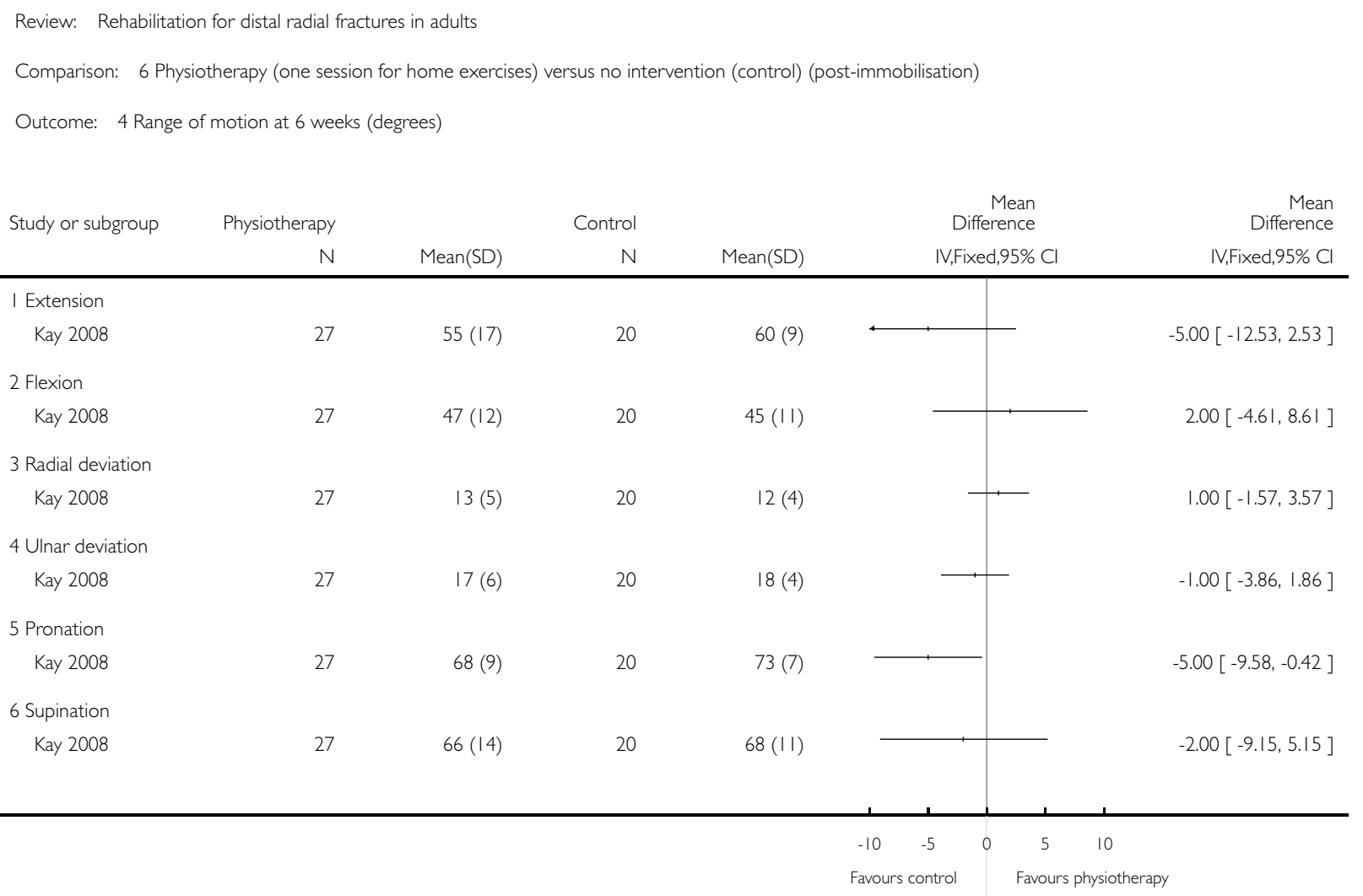


Analysis 6.5. Comparison 6 Physiotherapy (one session for home exercises) versus no intervention (control) (post-immobilisation), Outcome 5 Change in range of motion at 6 weeks (degrees).

Review: Rehabilitation for distal radial fractures in adults

Comparison: 6 Physiotherapy (one session for home exercises) versus no intervention (control) (post-immobilisation)

Outcome: 5 Change in range of motion at 6 weeks (degrees)

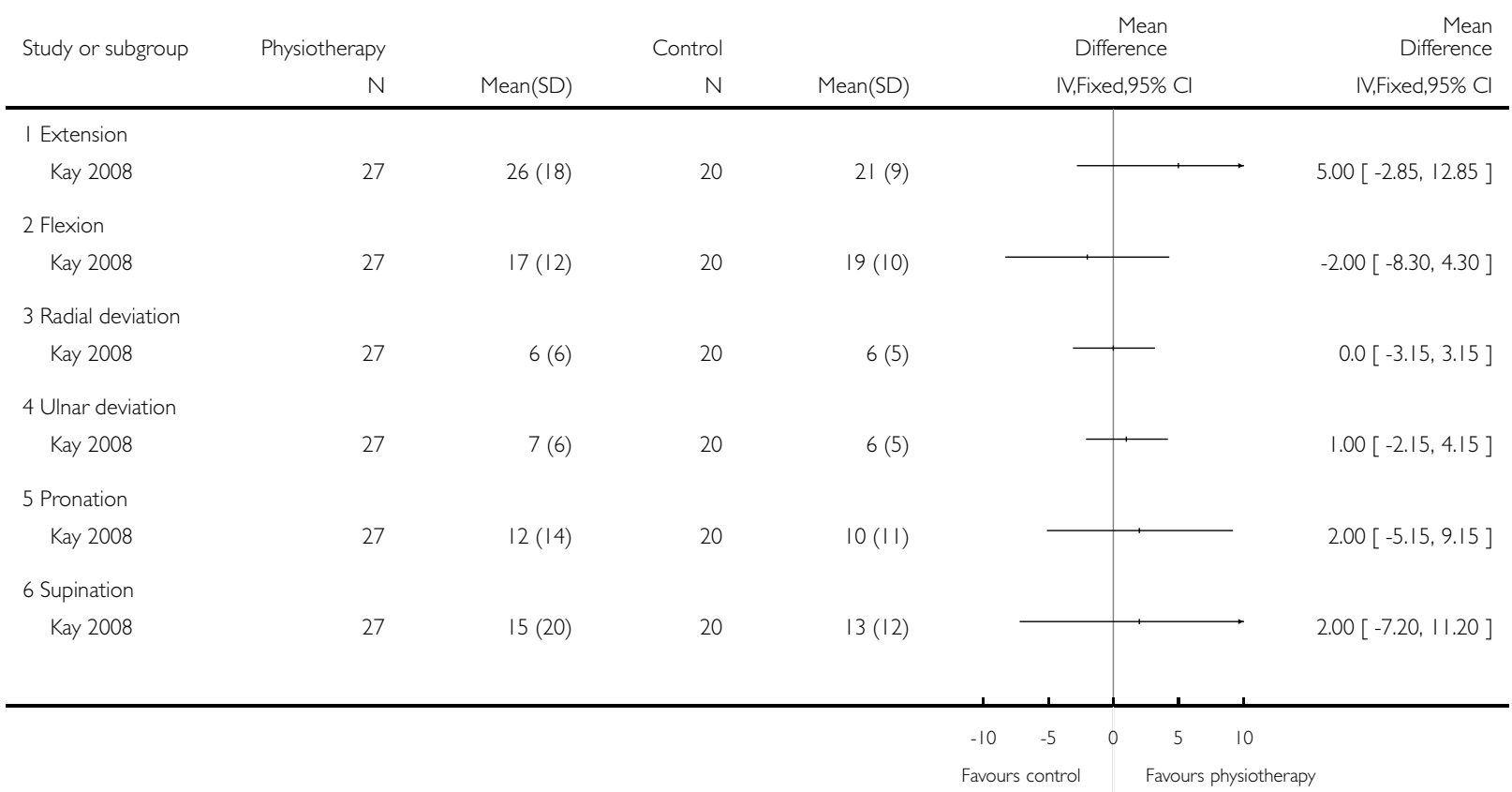


Analysis 6.6. Comparison 6 Physiotherapy (one session for home exercises) versus no intervention (control) (post-immobilisation), Outcome 6 Complications.

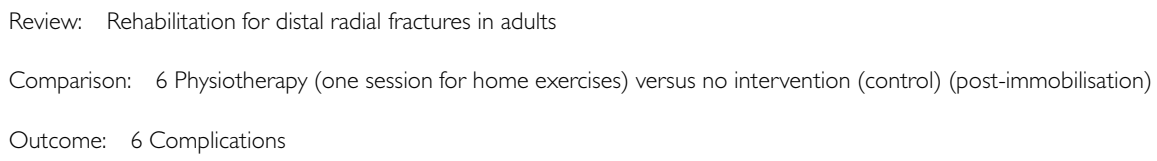

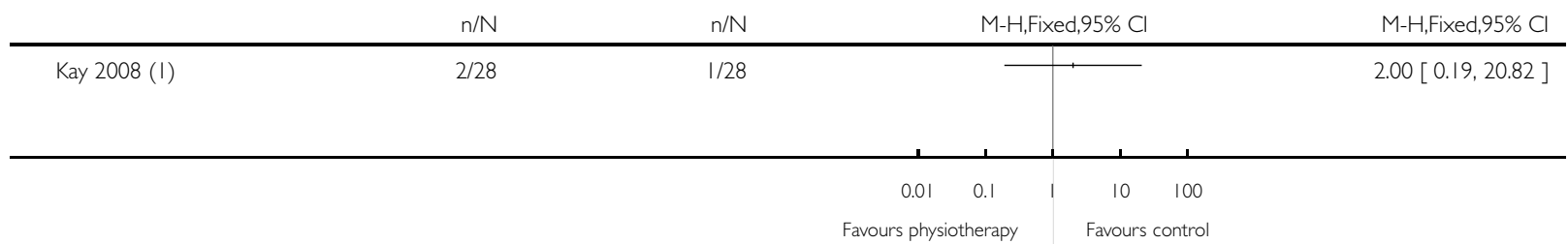

( I) Physiotherapy: I scar and I carpal tunnel syndrome; Control: I instability

Analysis 6.7. Comparison 6 Physiotherapy (one session for home exercises) versus no intervention (control) (post-immobilisation), Outcome 7 Request for more physiotherapy.

Review: Rehabilitation for distal radial fractures in adults

Comparison: 6 Physiotherapy (one session for home exercises) versus no intervention (control) (post-immobilisation)

Outcome: 7 Request for more physiotherapy

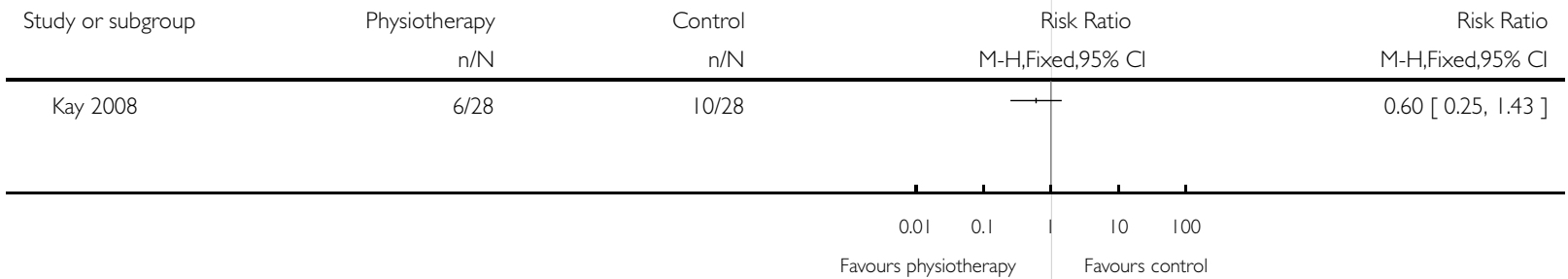


Analysis 7.I. Comparison 7 Post-immobilisation occupational or physiotherapy versus no intervention (control), Outcome I Patient-Rated Wrist Evaluation (PRWE) at 24 weeks (\%: 100\% = worst results).

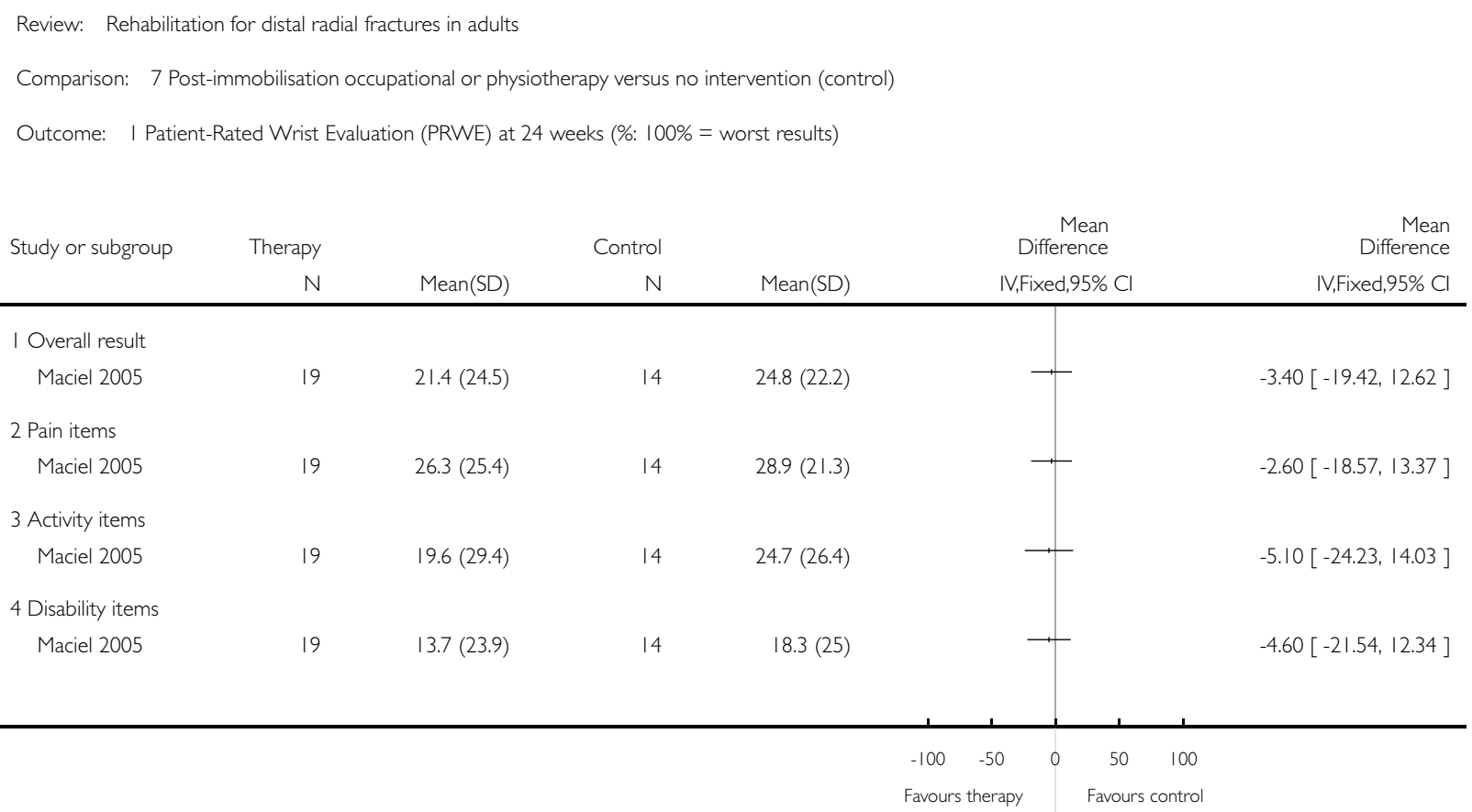


Analysis 7.2. Comparison 7 Post-immobilisation occupational or physiotherapy versus no intervention (control), Outcome 2 Activities of daily living scores (\% of unaffected side).

Review: Rehabilitation for distal radial fractures in adults

Comparison: 7 Post-immobilisation occupational or physiotherapy versus no intervention (control)

Outcome: 2 Activities of daily living scores (\% of unaffected side)

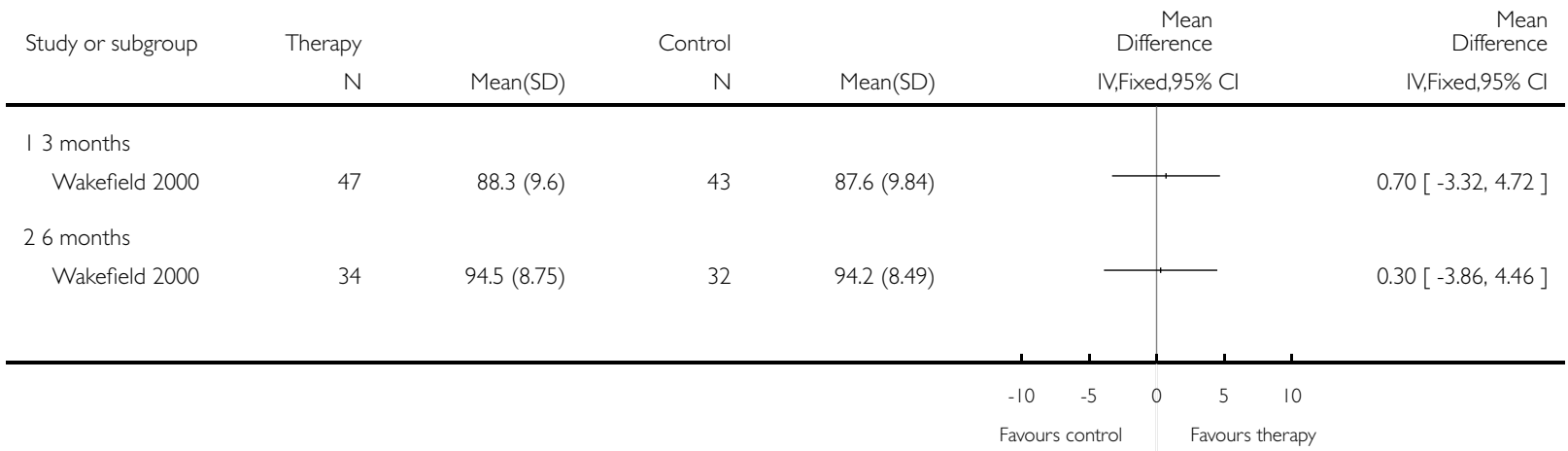

Analysis 7.3. Comparison 7 Post-immobilisation occupational or physiotherapy versus no intervention (control), Outcome 3 Grip strength (kg).

Review: Rehabilitation for distal radial fractures in adults

Comparison: 7 Post-immobilisation occupational or physiotherapy versus no intervention (control)

Outcome: 3 Grip strength $(\mathrm{kg})$

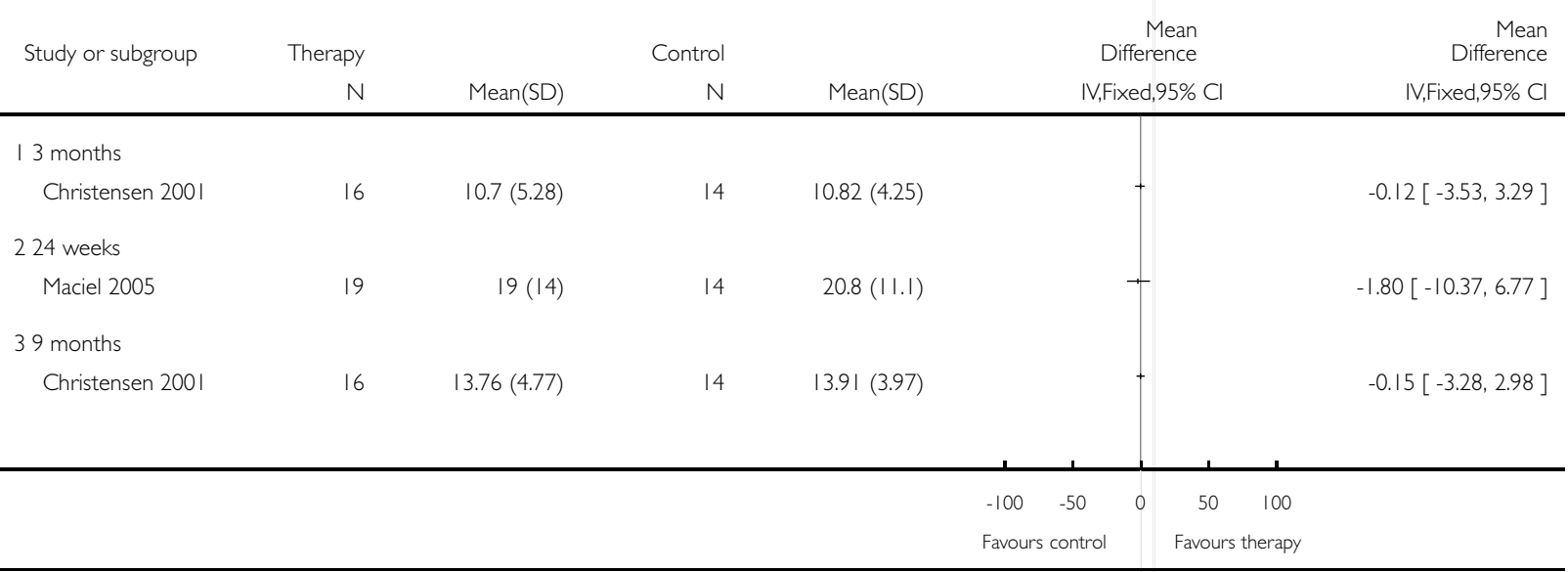

Rehabilitation for distal radial fractures in adults (Review)

Copyright @ 2015 The Cochrane Collaboration. Published by John Wiley \& Sons, Ltd. 
Analysis 7.4. Comparison 7 Post-immobilisation occupational or physiotherapy versus no intervention (control), Outcome 4 Grip strength (\% of unaffected side).

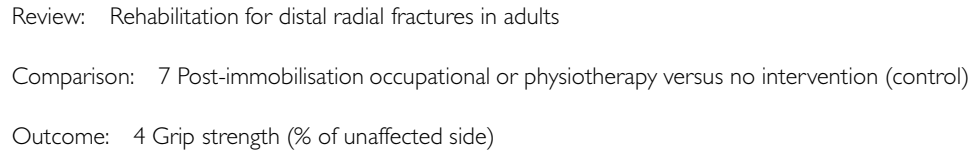

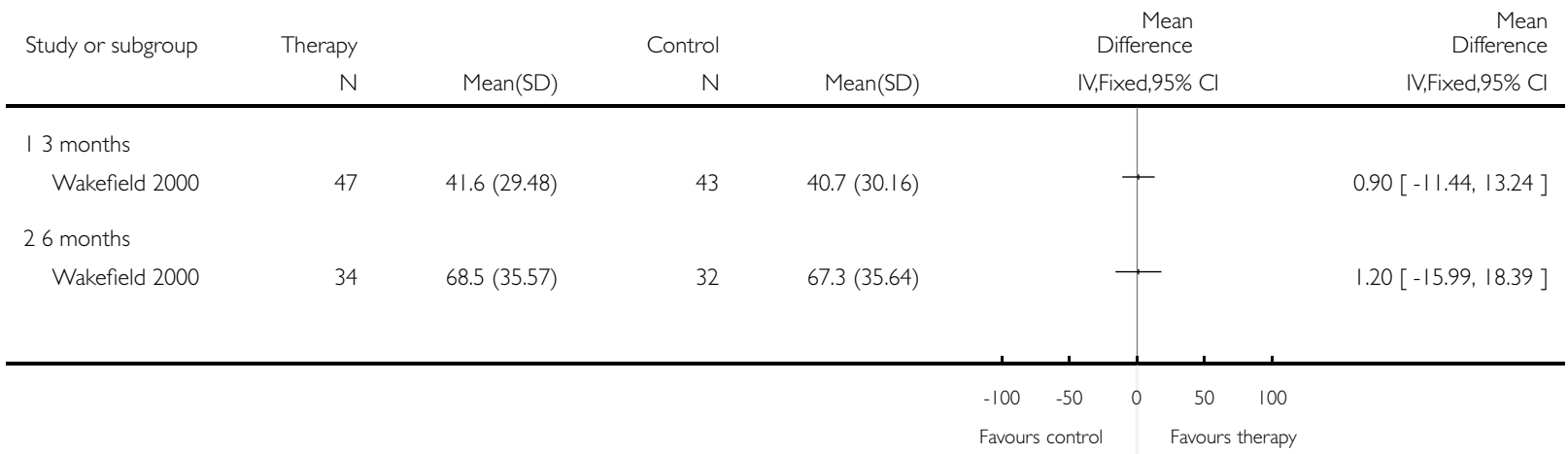


Analysis 7.5. Comparison 7 Post-immobilisation occupational or physiotherapy versus no intervention (control), Outcome 5 Pain (VAS: none to worst imaginable at $10 \mathrm{~cm}$ ).

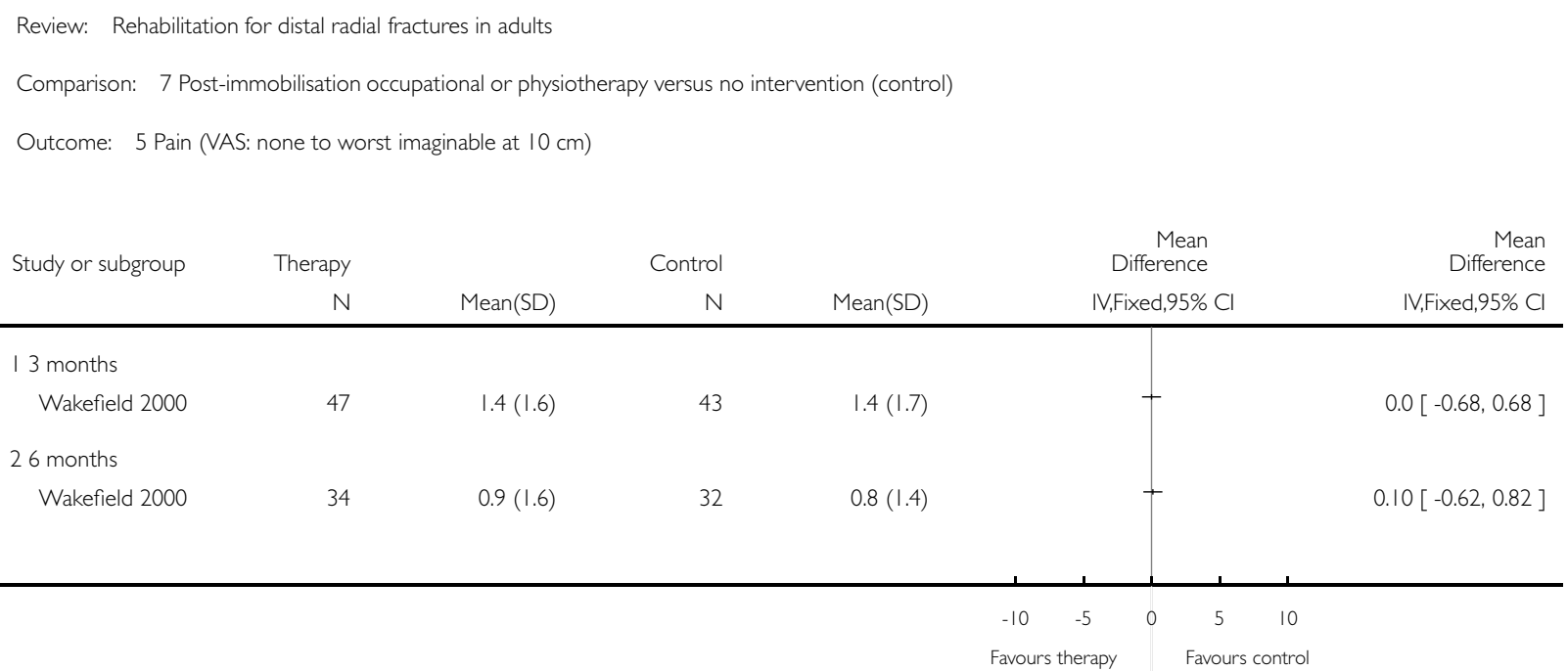

Analysis 7.6. Comparison 7 Post-immobilisation occupational or physiotherapy versus no intervention (control), Outcome 6 Range of motion at 24 weeks.

Review: Rehabilitation for distal radial fractures in adults

Comparison: 7 Post-immobilisation occupational or physiotherapy versus no intervention (control)

Outcome: 6 Range of motion at 24 weeks

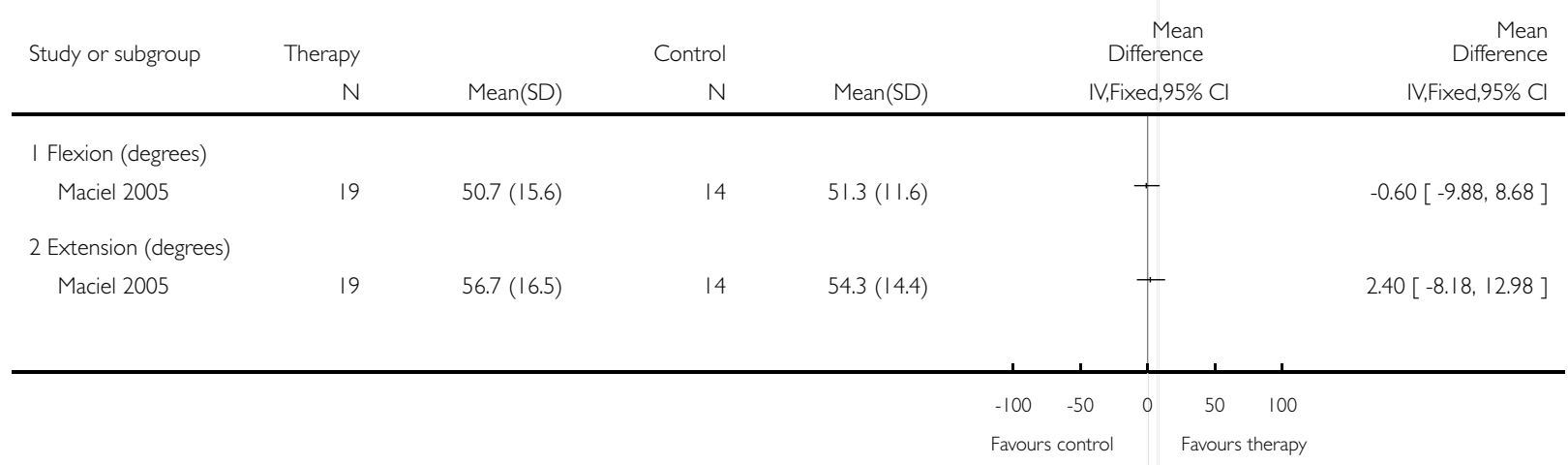


Analysis 7.7. Comparison 7 Post-immobilisation occupational or physiotherapy versus no intervention (control), Outcome 7 Range of motion (\% of unaffected side) at 3 months.

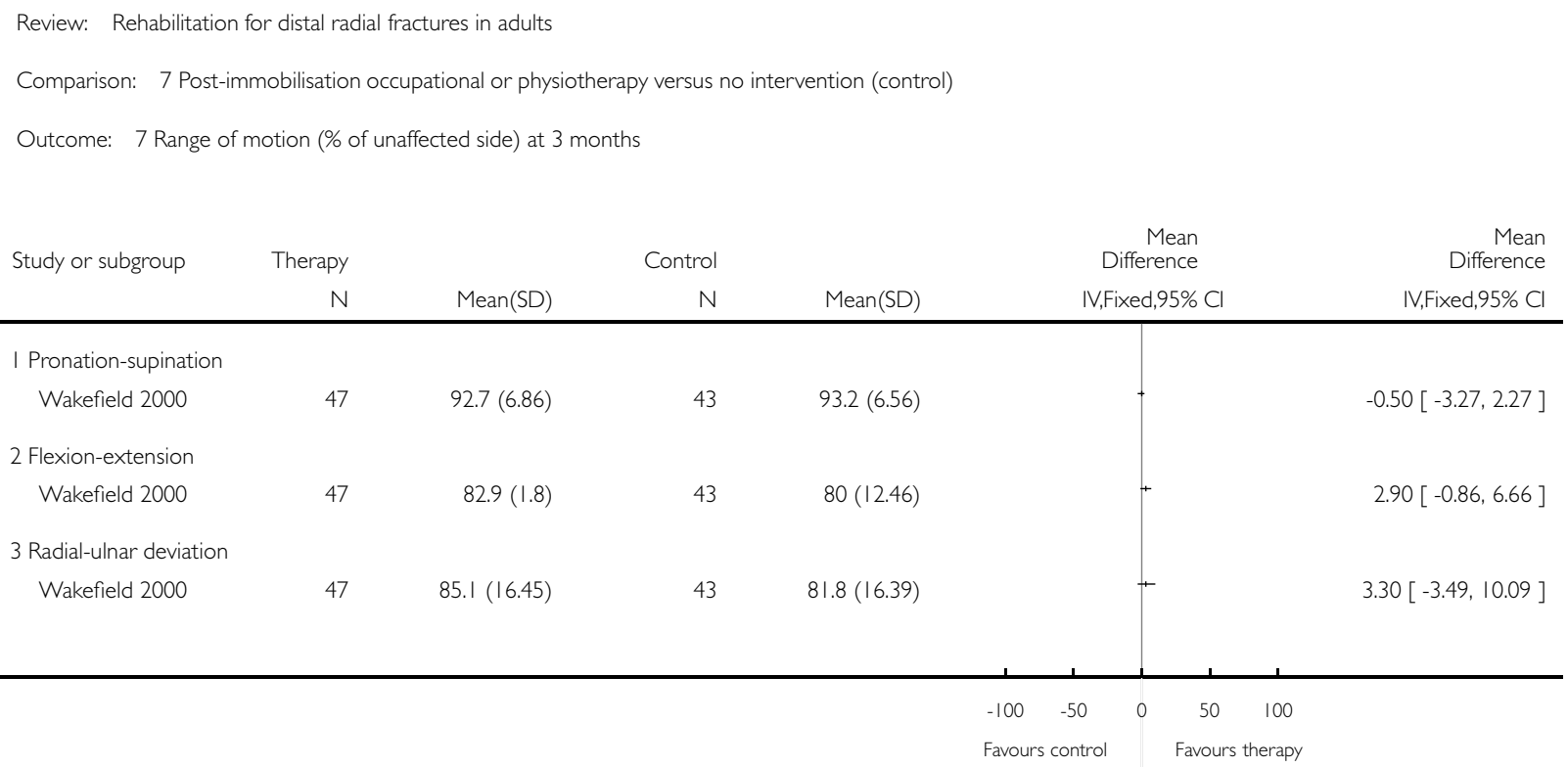


Analysis 7.8. Comparison 7 Post-immobilisation occupational or physiotherapy versus no intervention (control), Outcome 8 Range of motion (\% of unaffected side) at 6 months.

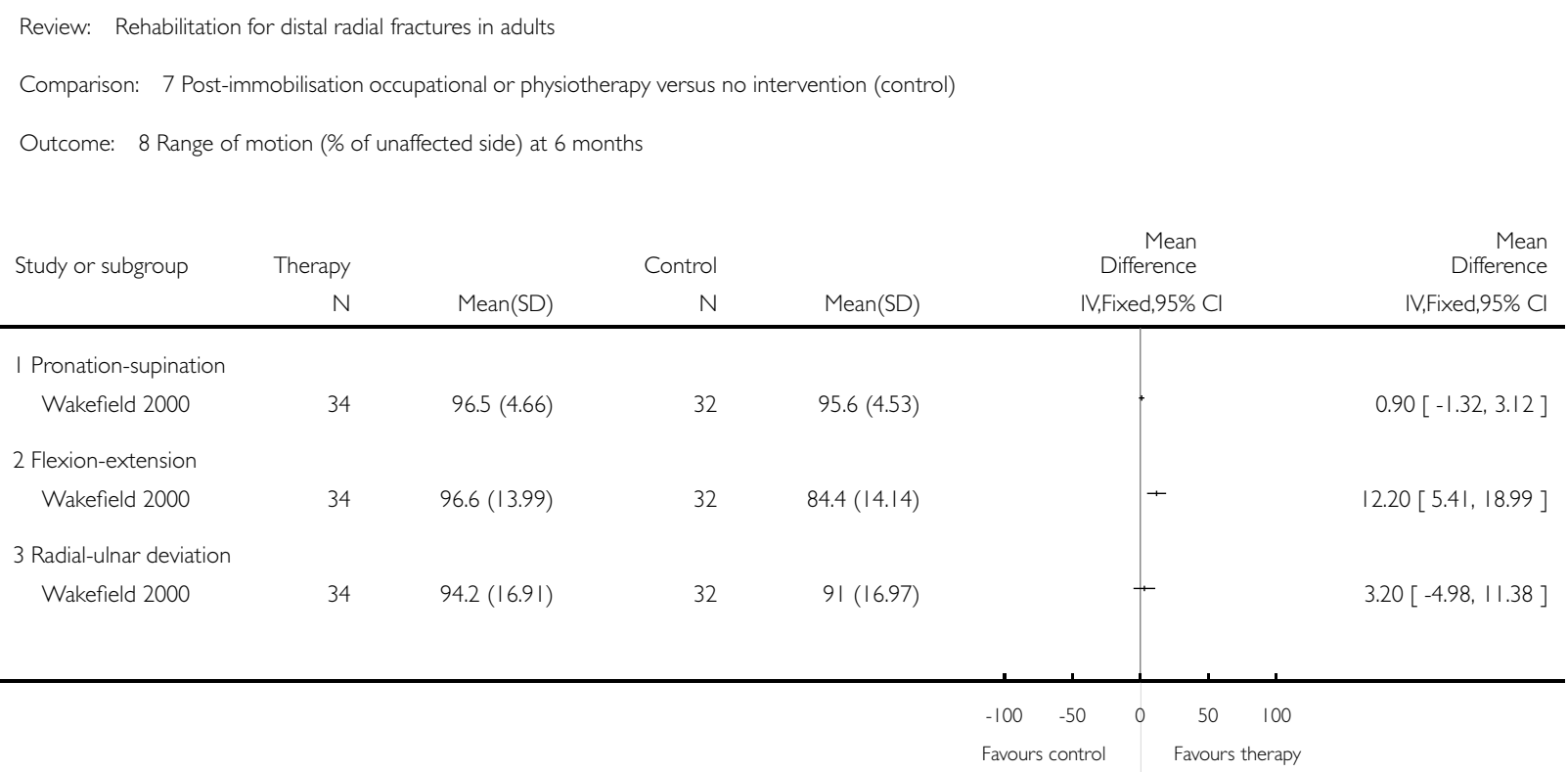

Analysis 7.9. Comparison 7 Post-immobilisation occupational or physiotherapy versus no intervention (control), Outcome 9 Number of treatments.

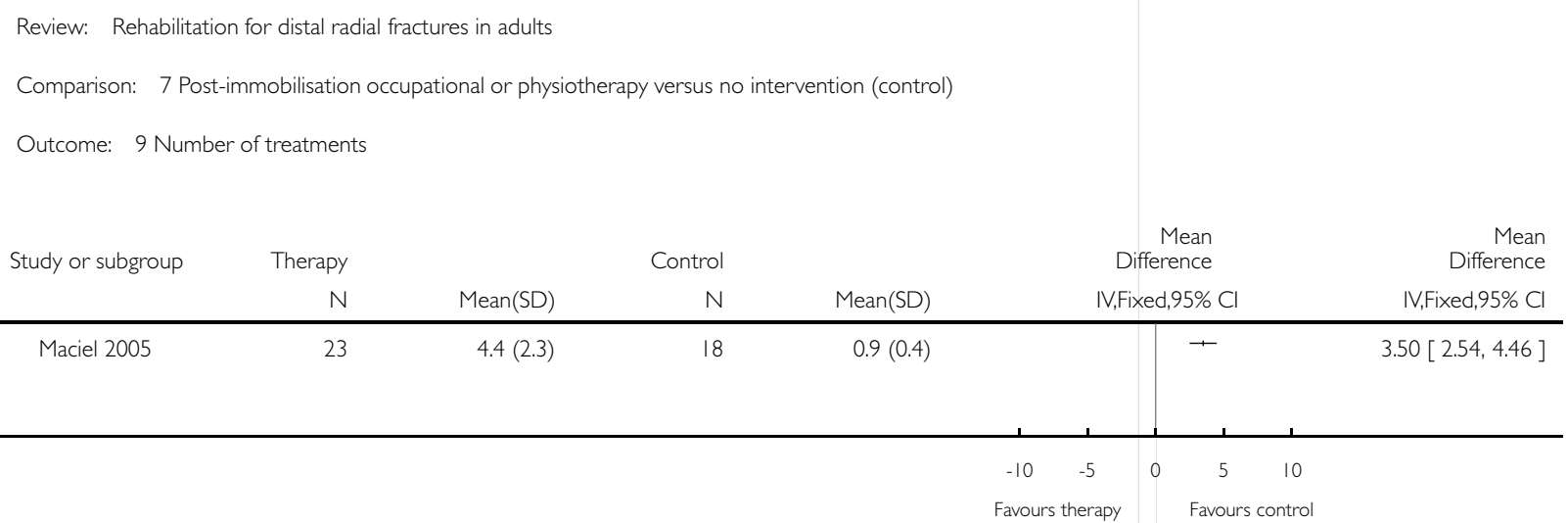


Analysis 7.10. Comparison 7 Post-immobilisation occupational or physiotherapy versus no intervention (control), Outcome 10 Complications.

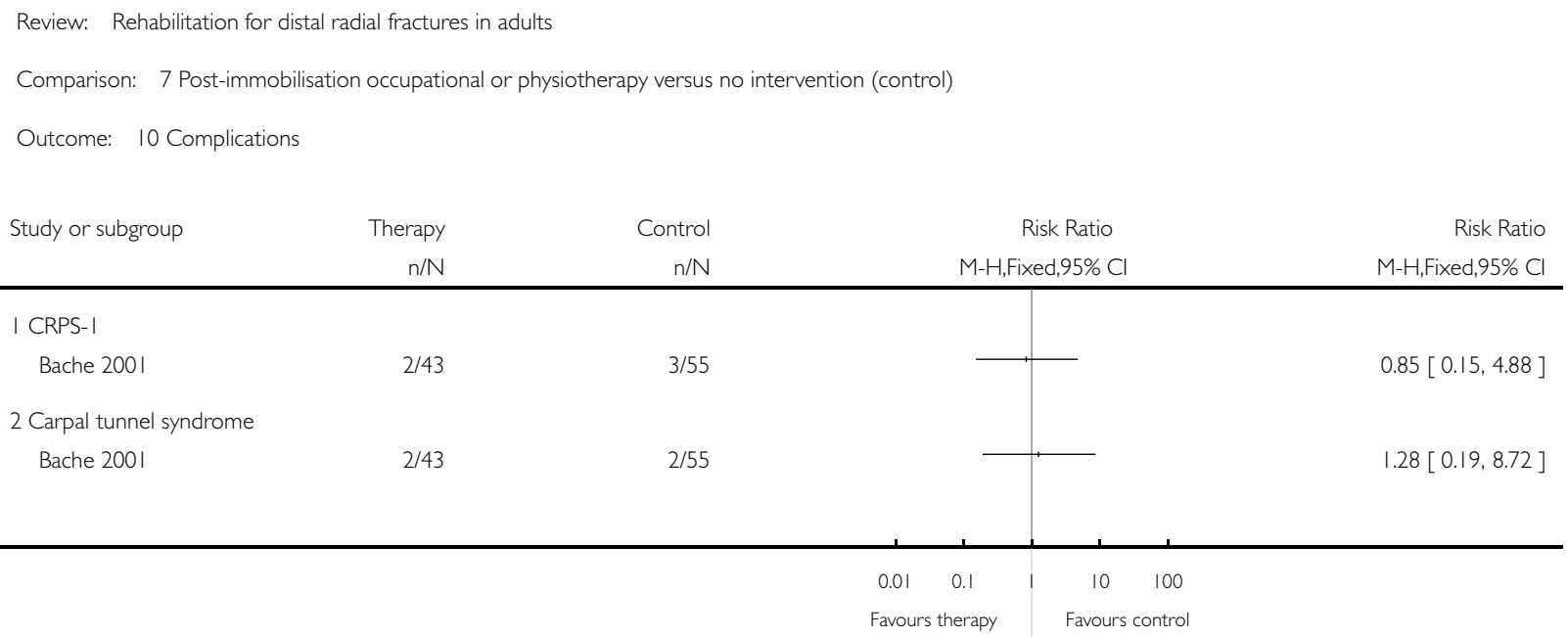

Analysis 8.1. Comparison 8 Continuous passive motion (CPM) (post-external fixation) versus no intervention (control), Outcome I Time to recover independence (weeks).

Review: Rehabilitation for distal radial fractures in adults

Comparison: 8 Continuous passive motion (CPM) (post-external fixation) versus no intervention (control)

Outcome: I Time to recover independence (weeks)

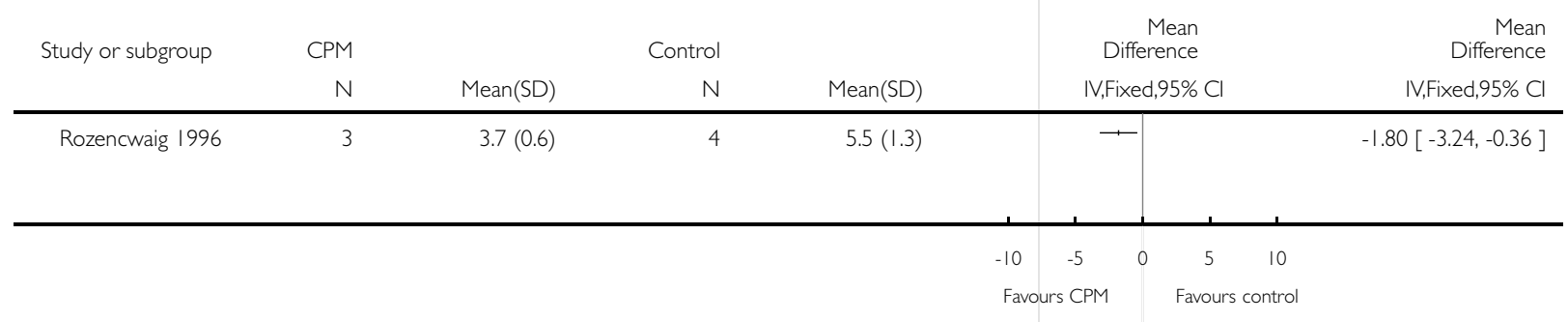


Analysis 9.1. Comparison 9 Pulsed electromagnetic field (PEMF) (post-immobilisation) versus sham control, Outcome I Pain and volume at day 5.

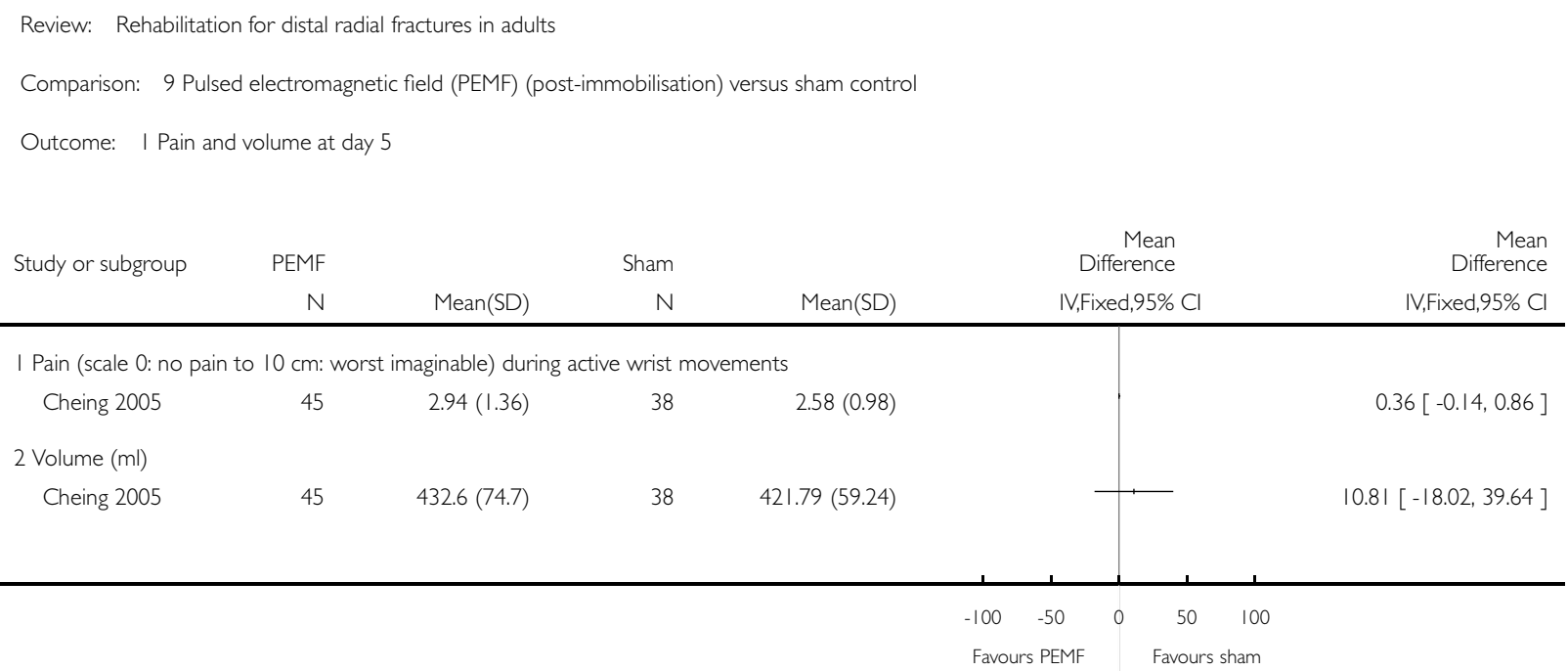


Analysis 9.2. Comparison 9 Pulsed electromagnetic field (PEMF) (post-immobilisation) versus sham control, Outcome 2 Range of motion at day 5.

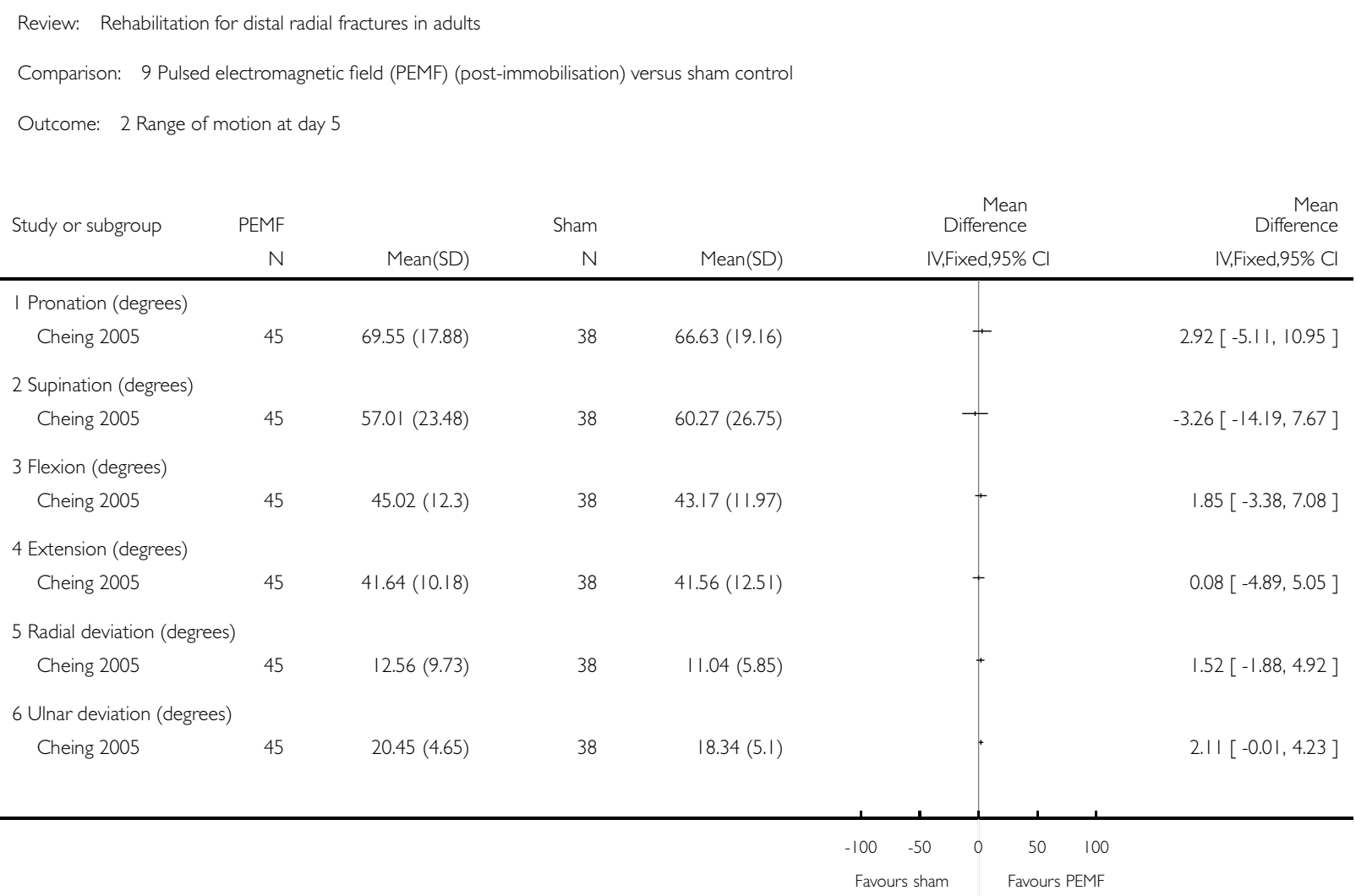


Analysis 10.1. Comparison I0 Ice (post-immobilisation) versus no ice (control), Outcome I Pain and volume at day 5 .

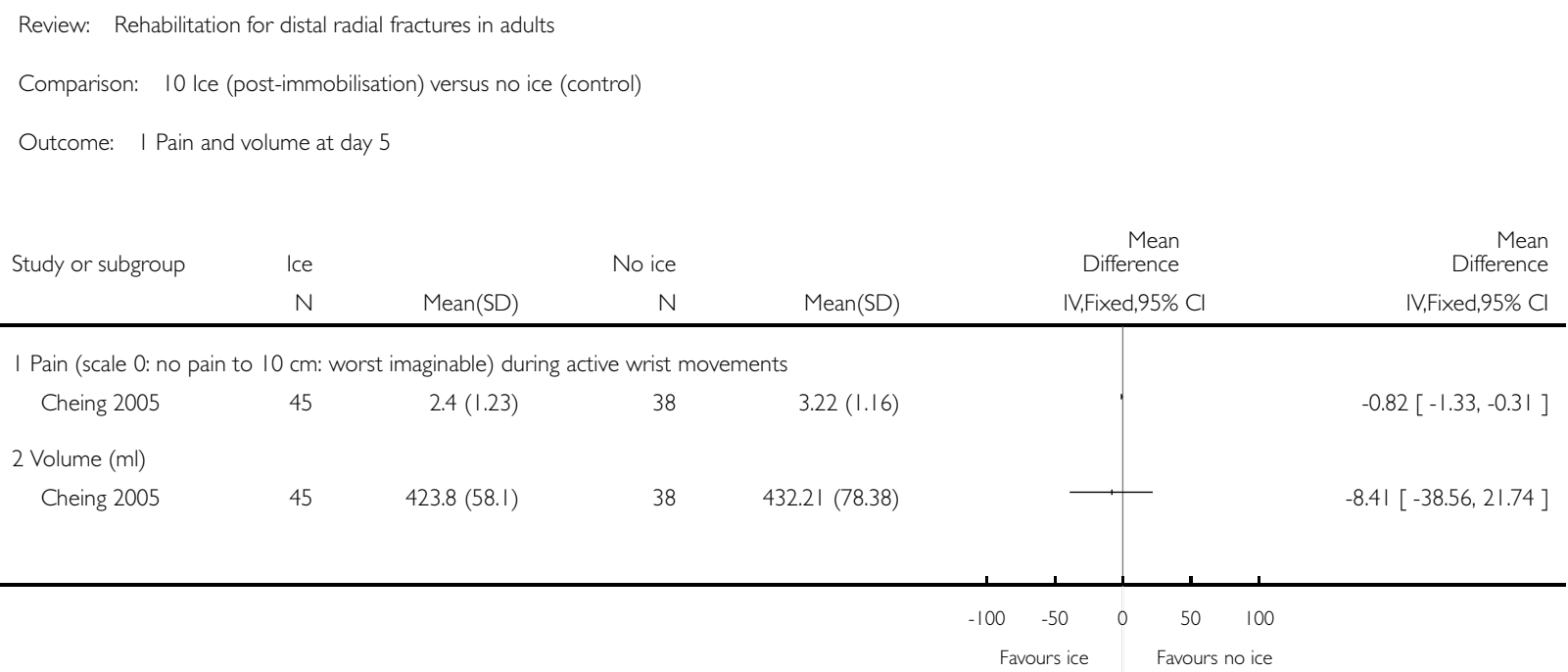


Analysis 10.2. Comparison 10 Ice (post-immobilisation) versus no ice (control), Outcome 2 Range of motion at day 5 .

Review: Rehabilitation for distal radial fractures in adults

Comparison: 10 Ice (post-immobilisation) versus no ice (control)

Outcome: 2 Range of motion at day 5

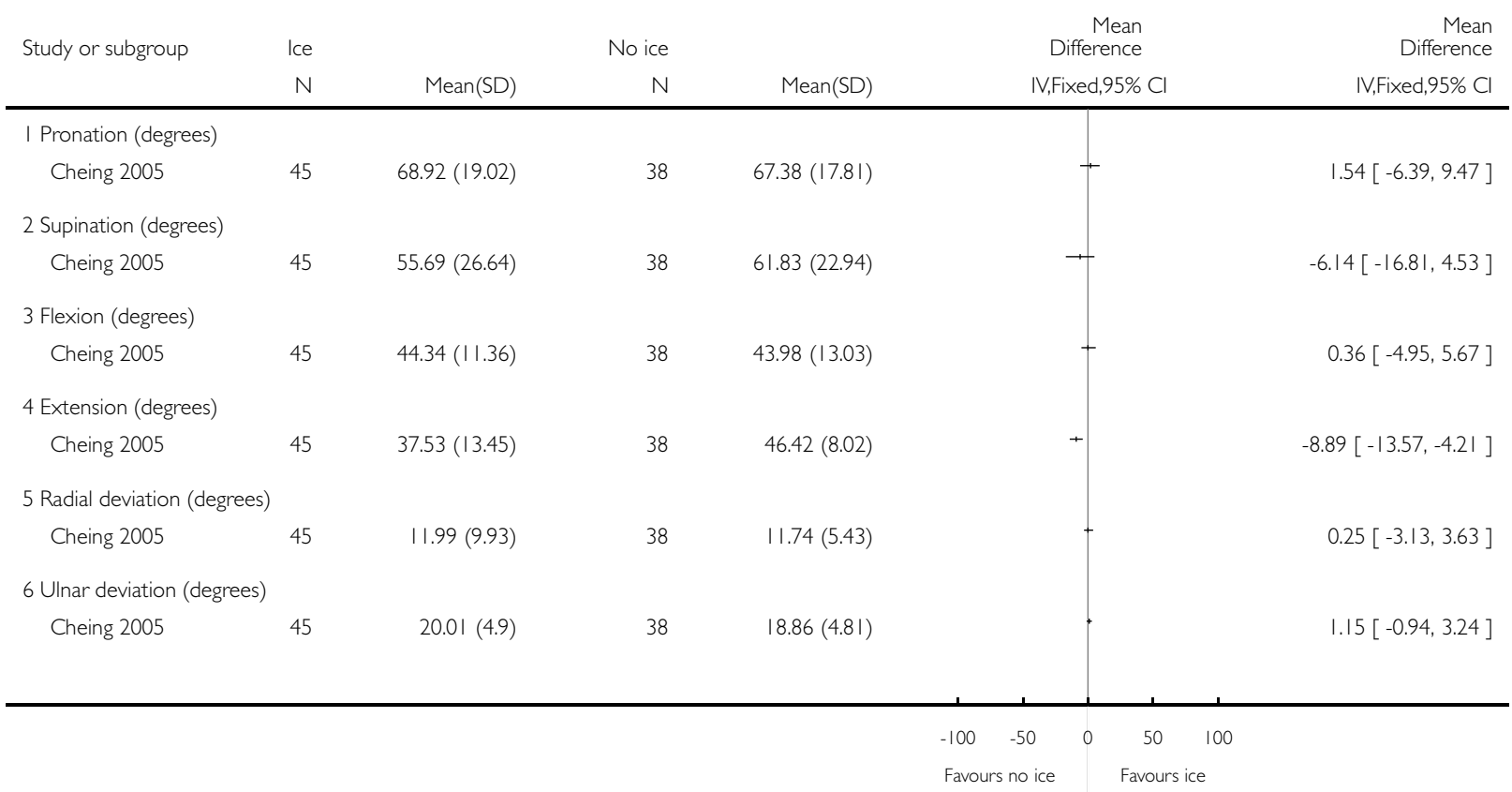


Analysis I I.I. Comparison I I Pulsed electromagnetic field (PEMF) plus ice (post-immobilisation) versus no intervention (control), Outcome I Pain and volume at day 5.

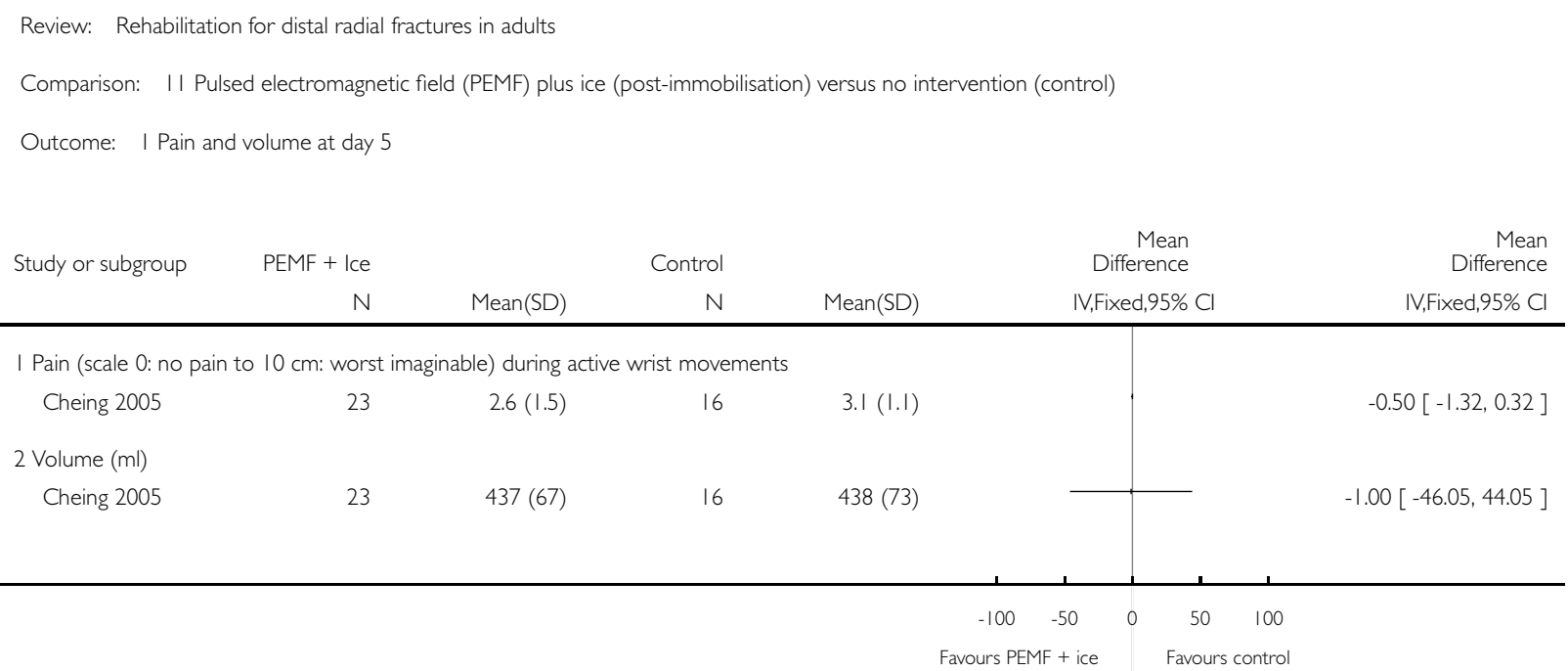


Analysis I I.2. Comparison I I Pulsed electromagnetic field (PEMF) plus ice (post-immobilisation) versus no intervention (control), Outcome 2 Range of motion at day 5.

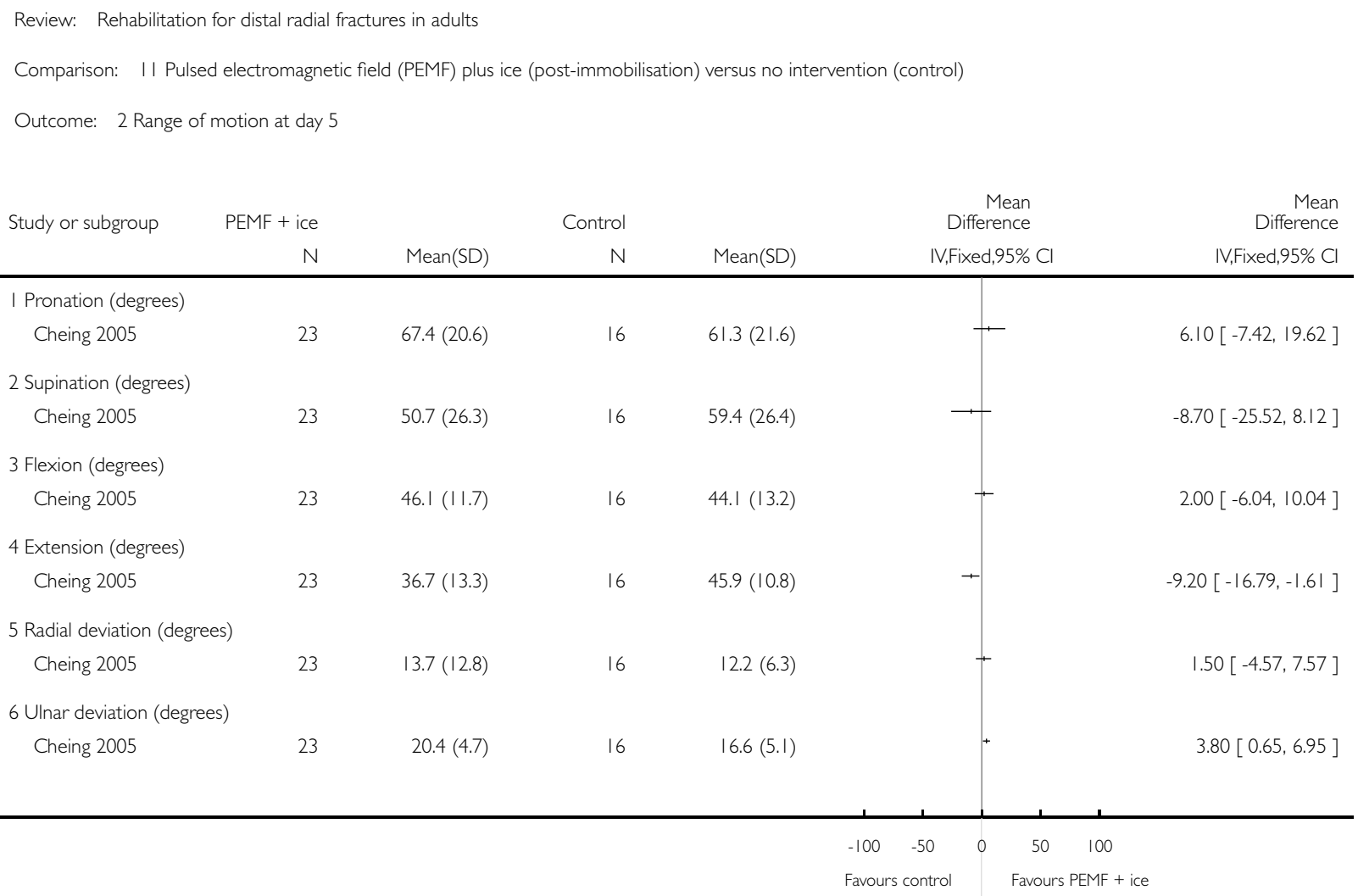




\section{Analysis 12.I. Comparison I 2 Passive mobilisation (post-immobilisation) versus no intervention (control),} Outcome I Grip strength $(\mathbf{k g})$ at 6 weeks.

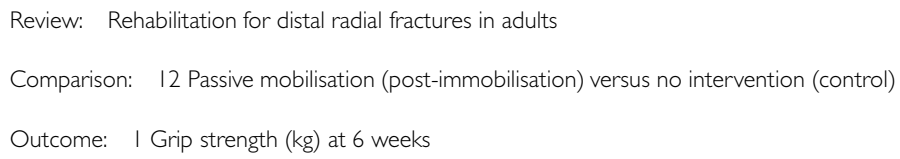

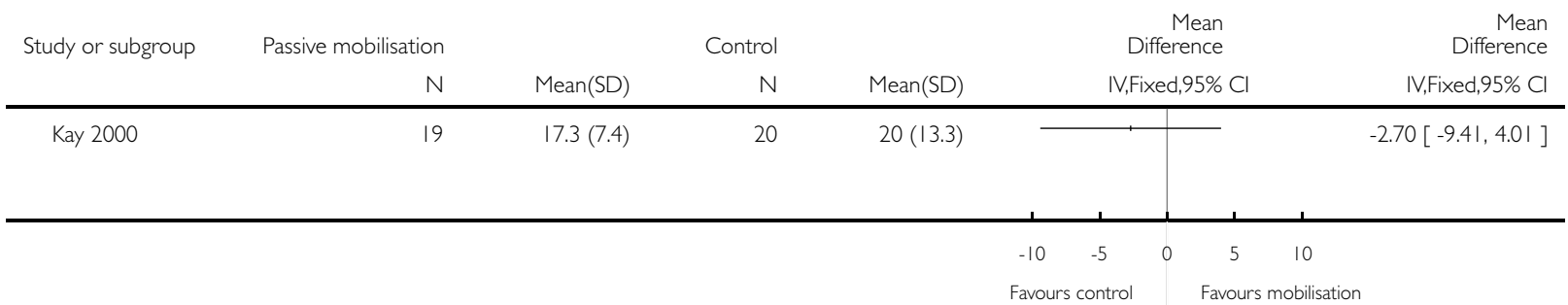

Analysis 12.2. Comparison I2 Passive mobilisation (post-immobilisation) versus no intervention (control), Outcome 2 Range of motion at 6 weeks.

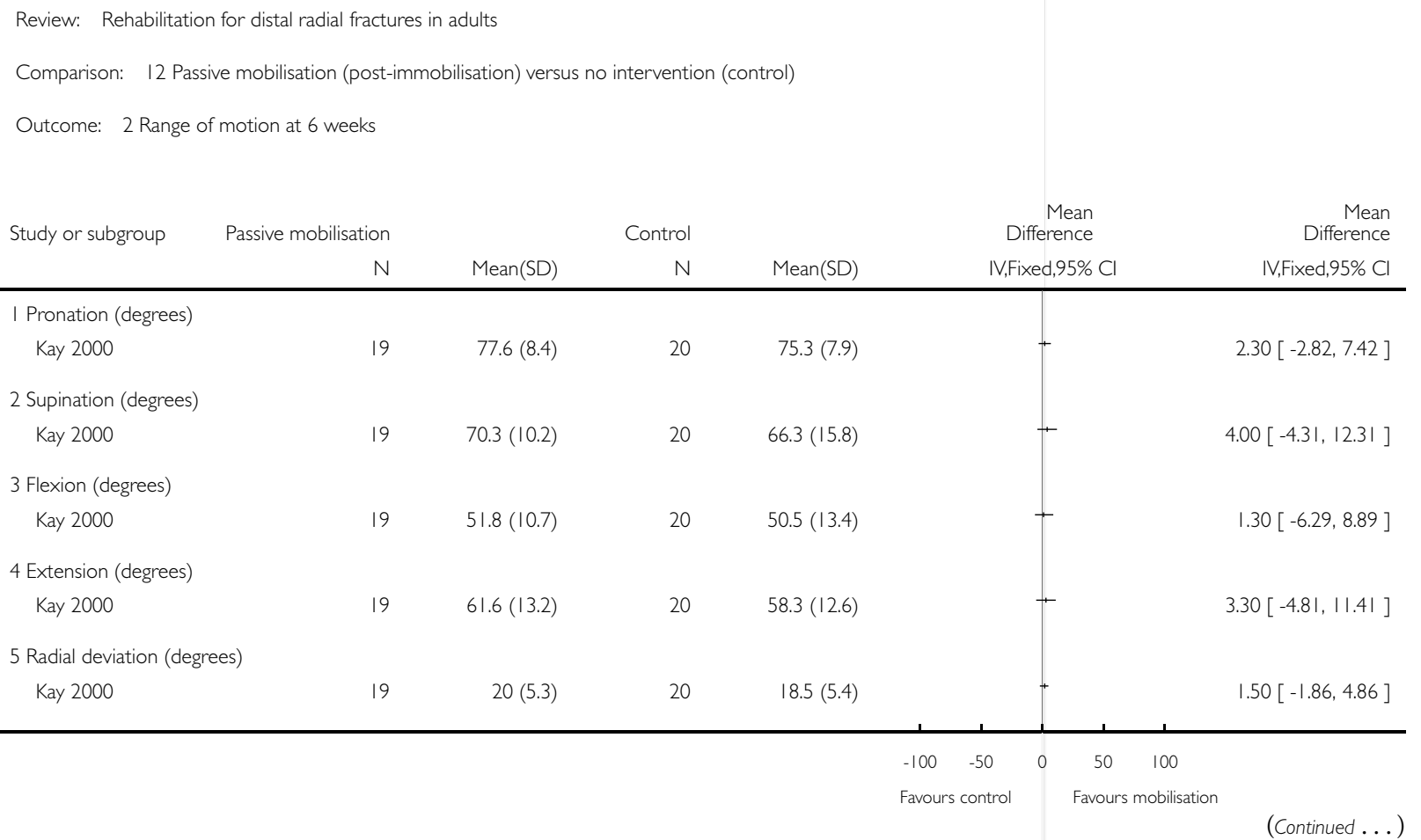




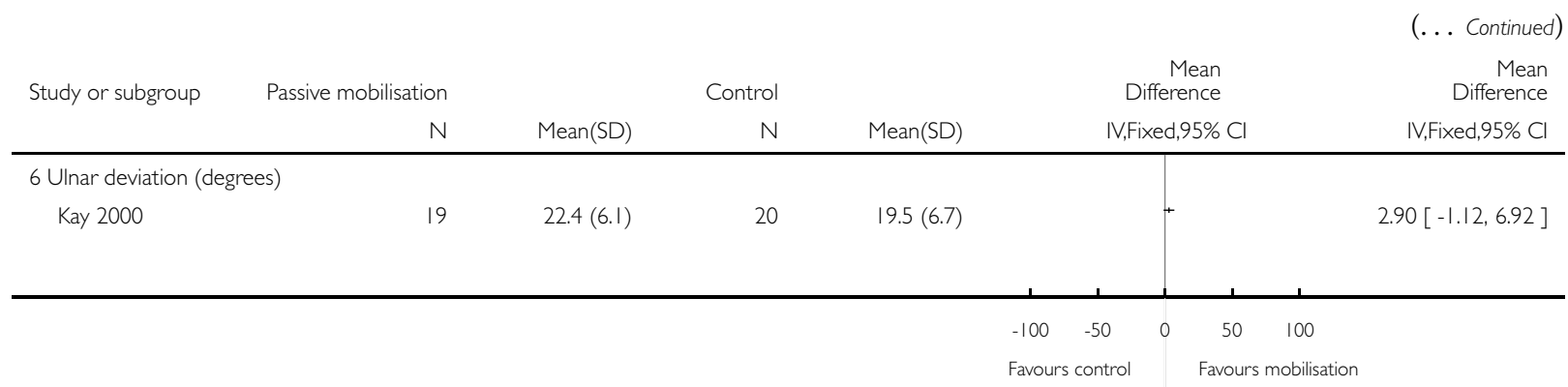

Analysis 12.3. Comparison I 2 Passive mobilisation (post-immobilisation) versus no intervention (control), Outcome 3 Web space angle (degrees) at 6 weeks.

Review: Rehabilitation for distal radial fractures in adults

Comparison: 12 Passive mobilisation (post-immobilisation) versus no intervention (control)

Outcome: 3 Web space angle (degrees) at 6 weeks

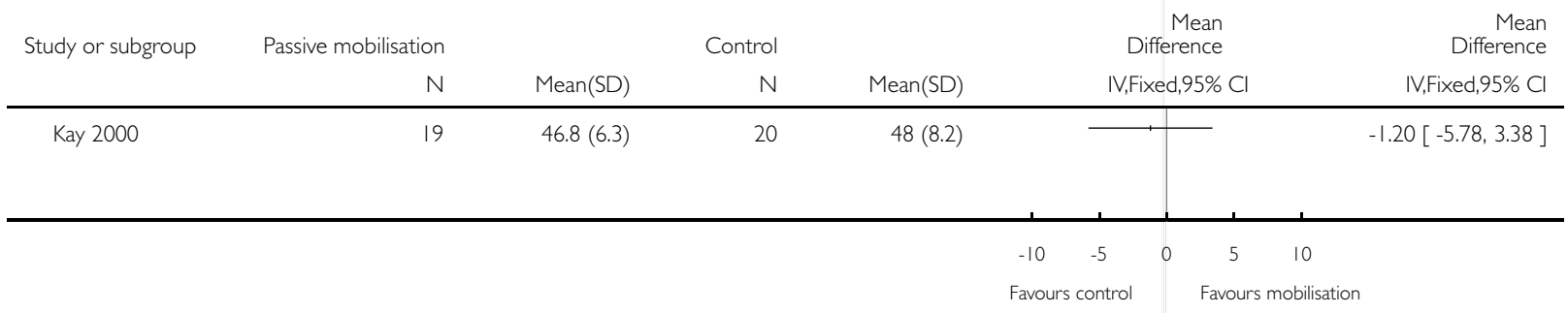




\section{Analysis 12.4. Comparison I 2 Passive mobilisation (post-immobilisation) versus no intervention (control),} Outcome 4 Wrist extension at discharge (4 weeks).

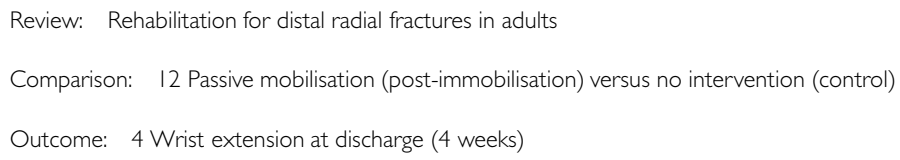

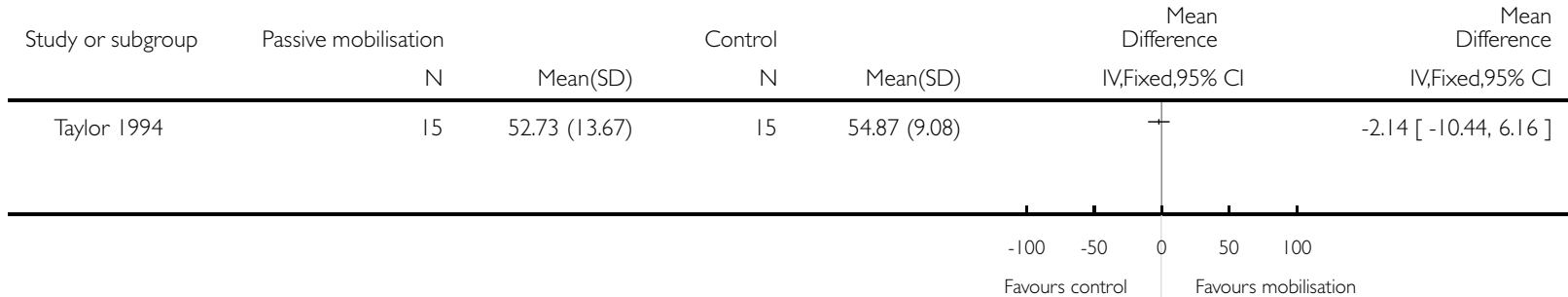

Analysis 12.5. Comparison I2 Passive mobilisation (post-immobilisation) versus no intervention (control), Outcome 5 Number of treatments.

Review: Rehabilitation for distal radial fractures in adults

Comparison: 12 Passive mobilisation (post-immobilisation) versus no intervention (control)

Outcome: 5 Number of treatments

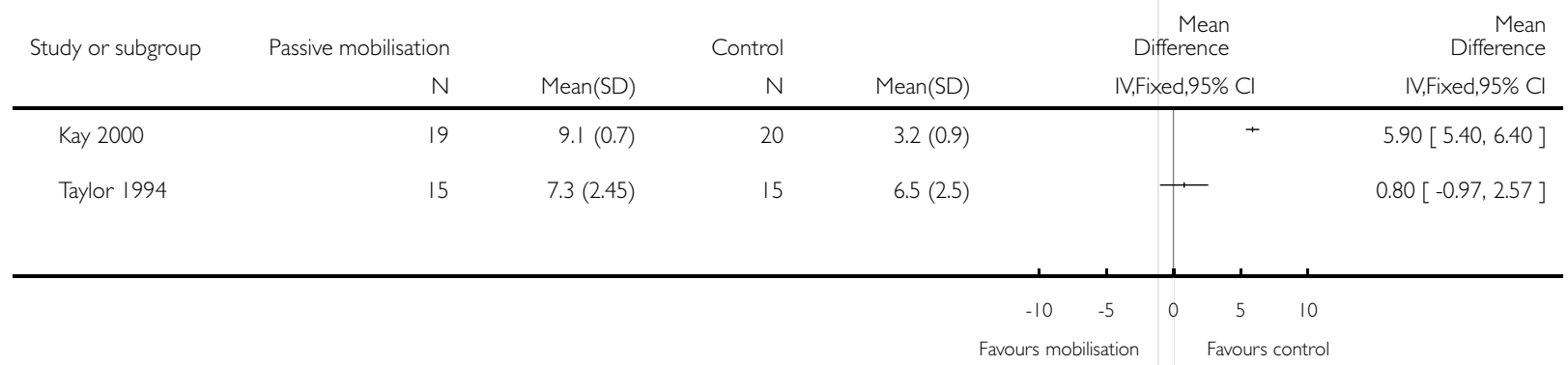




\section{Analysis 12.6. Comparison I 2 Passive mobilisation (post-immobilisation) versus no intervention (control),} Outcome 6 Time to discharge (days).

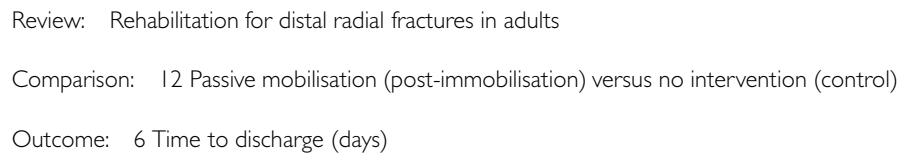

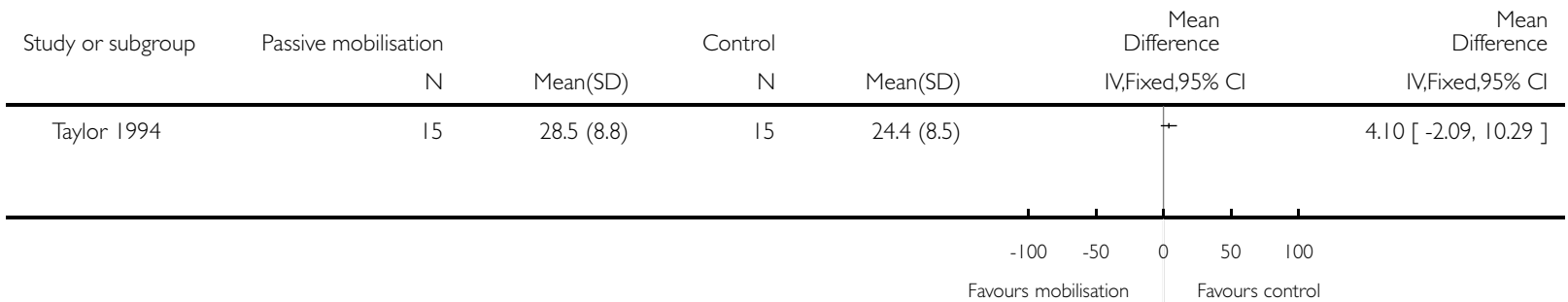

\section{Analysis 12.7. Comparison I 2 Passive mobilisation (post-immobilisation) versus no intervention (control),} Outcome 7 Complications at 6 weeks.

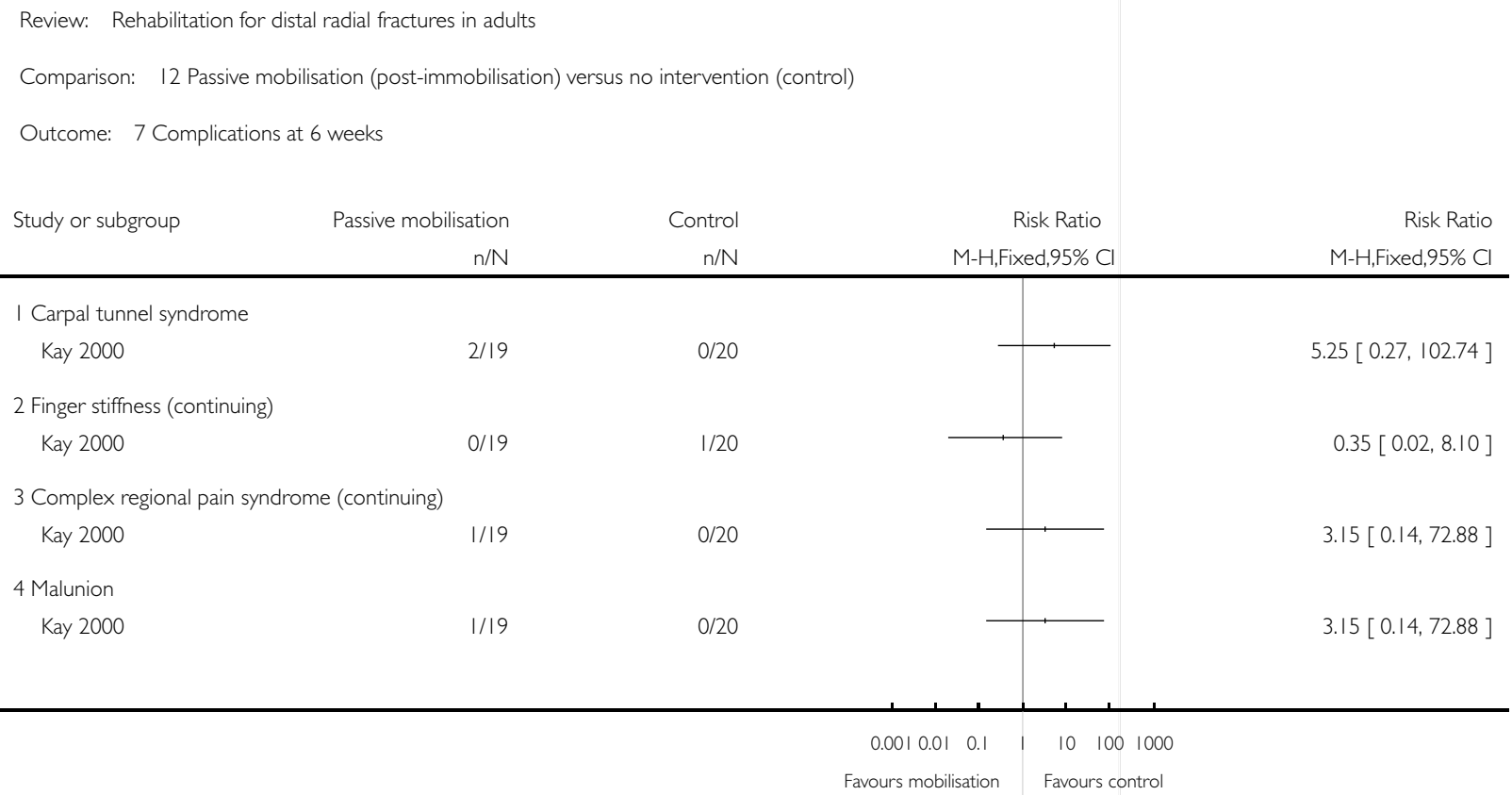


Analysis 13.I. Comparison I 3 Low frequency, long-wave ultrasound (post-immobilisation) versus sham intervention, Outcome I Greater than $30 \%$ loss of wrist motion (flexion-extension) at 8 weeks.

Review: Rehabilitation for distal radial fractures in adults

Comparison: 13 Low frequency, long-wave ultrasound (post-immobilisation) versus sham intervention

Outcome: I Greater than $30 \%$ loss of wrist motion (flexion-extension) at 8 weeks

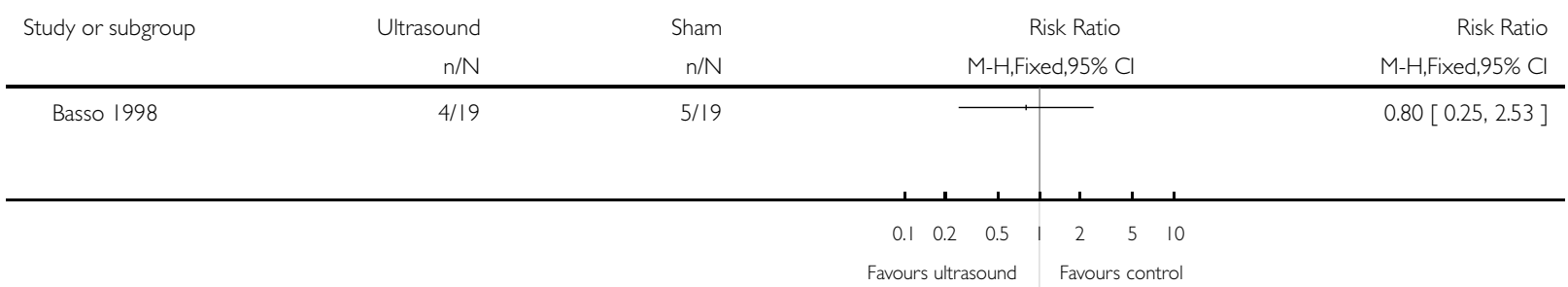

Analysis 13.2. Comparison 13 Low frequency, long-wave ultrasound (post-immobilisation) versus sham intervention, Outcome 2 Referral for physiotherapy.

Review: Rehabilitation for distal radial fractures in adults

Comparison: 13 Low frequency, long-wave ultrasound (post-immobilisation) versus sham intervention

Outcome: 2 Referral for physiotherapy

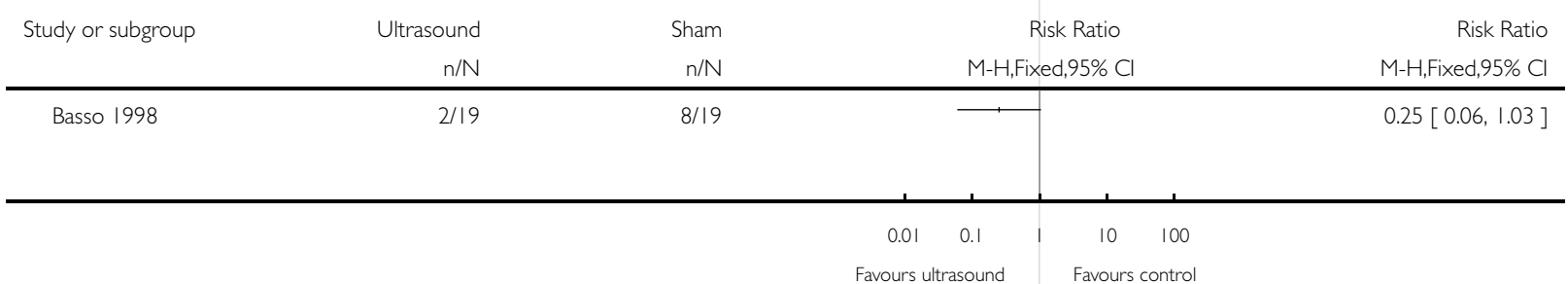


Analysis I4.I. Comparison I4 Whirlpool (post-immobilisation) versus towel (control), Outcome I Grip strength at end of treatment $(\mathrm{kg})$.

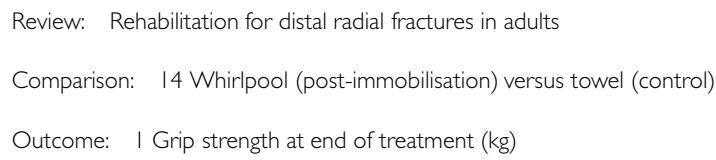

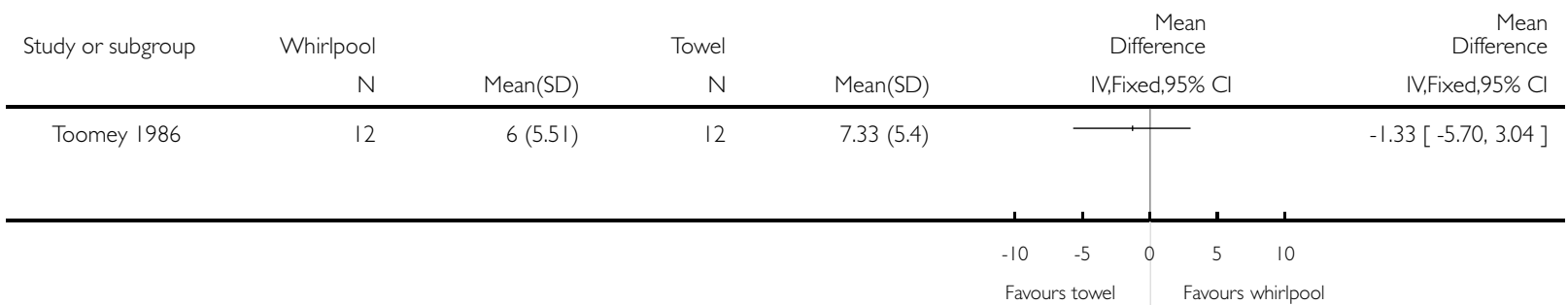

Analysis 14.2. Comparison I4 Whirlpool (post-immobilisation) versus towel (control), Outcome 2 Pain (scale 0: no pain to 5: excruciating) at end of treatment.

Review: Rehabilitation for distal radial fractures in adults

Comparison: 14 Whirlpool (post-immobilisation) versus towel (control)

Outcome: 2 Pain (scale 0: no pain to 5: excruciating) at end of treatment

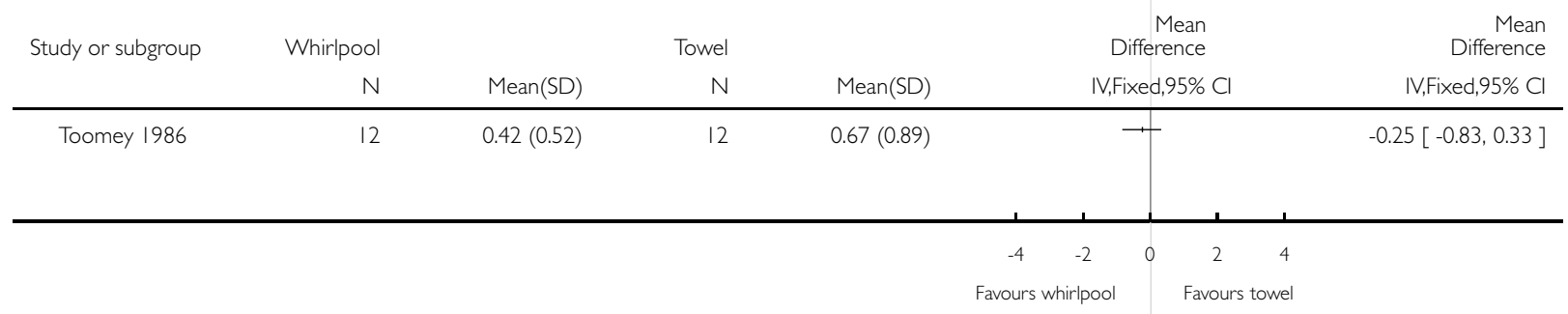


Analysis 14.3. Comparison I4 Whirlpool (post-immobilisation) versus towel (control), Outcome 3 Range of motion at end of treatment.

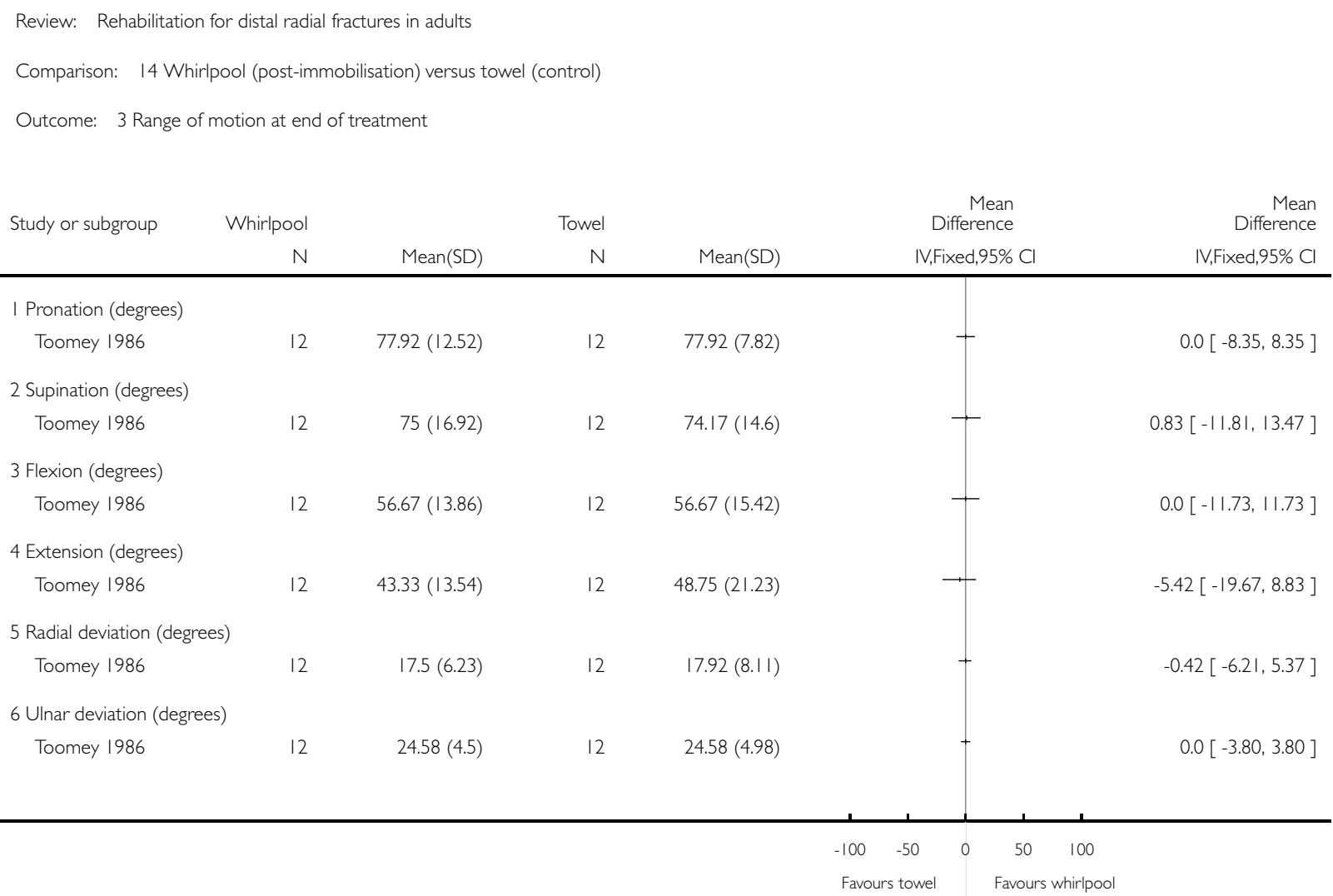


Analysis 14.4. Comparison I4 Whirlpool (post-immobilisation) versus towel (control), Outcome 4 Finger flexion at end of treatment.

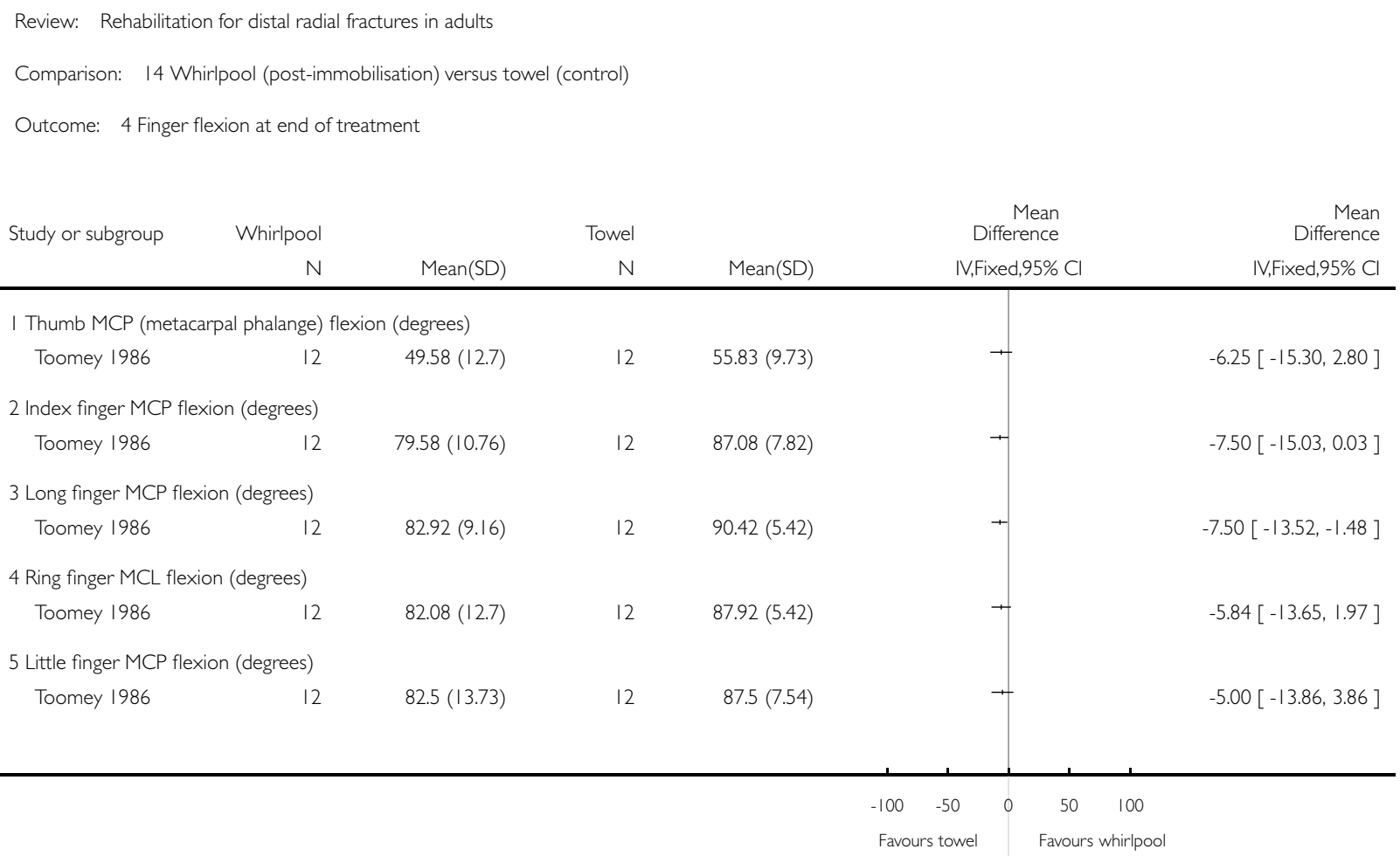


Analysis 14.5. Comparison I4 Whirlpool (post-immobilisation) versus towel (control), Outcome 5 Oedema (ml).

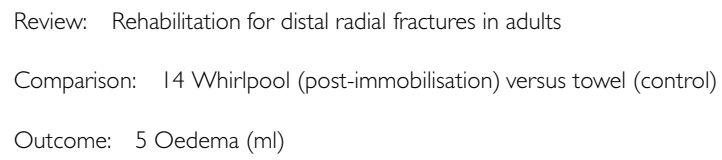

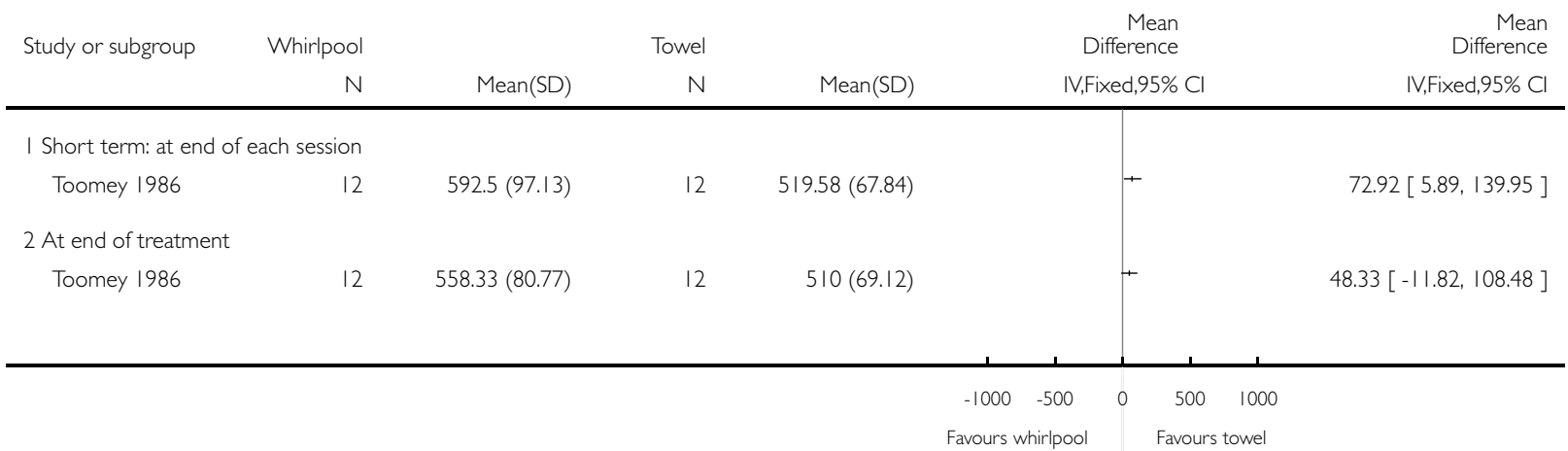

Analysis I5.I. Comparison I5 Dynamic wrist extension splint versus no intervention (control) (postimmobilisation), Outcome I Patient-Rated Wrist Evaluation (PRWE) (\%: 100\% = worst results).

Review: Rehabilitation for distal radial fractures in adults

Comparison: I5 Dynamic wrist extension splint versus no intervention (control) (post-immobilisation)

Outcome: I Patient-Rated Wrist Evaluation (PRWE) (\%: 100\% = worst results)

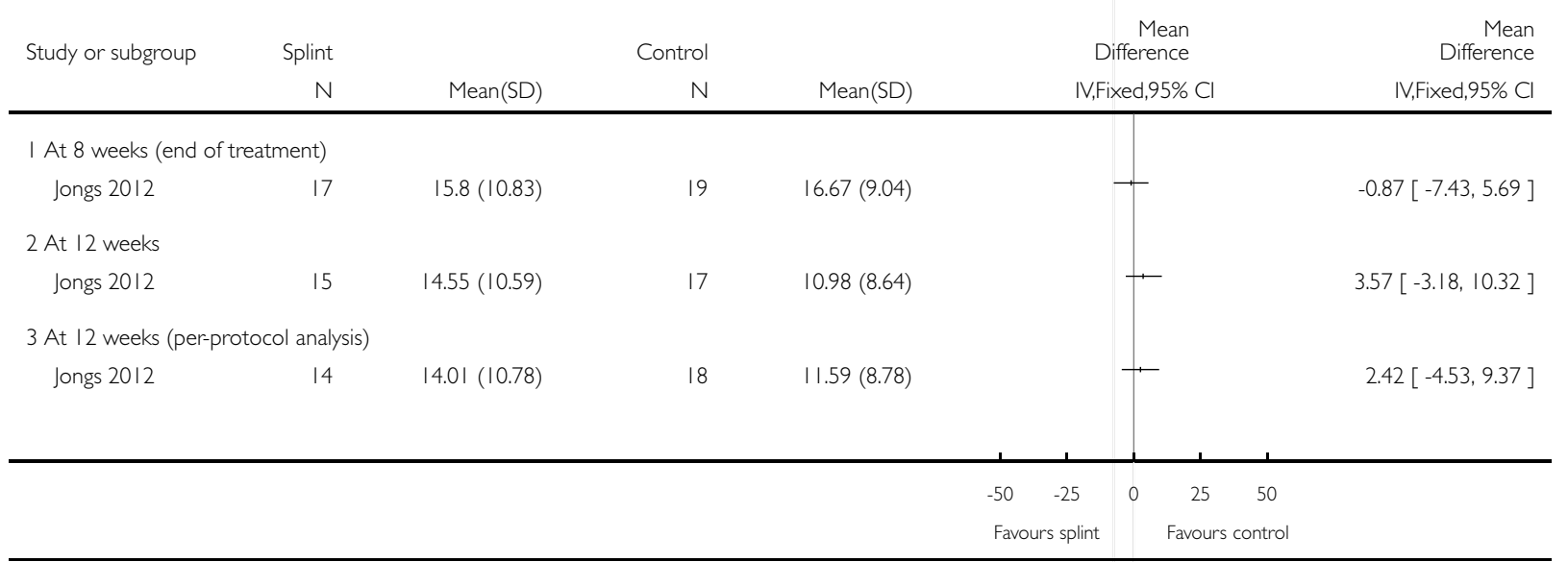

Rehabilitation for distal radial fractures in adults (Review) 
Analysis 15.2. Comparison I5 Dynamic wrist extension splint versus no intervention (control) (postimmobilisation), Outcome 2 Canadian Occupational Performance Measure at I 2 weeks.

Review: Rehabilitation for distal radial fractures in adults

Comparison: 15 Dynamic wrist extension splint versus no intervention (control) (post-immobilisation)

Outcome: 2 Canadian Occupational Performance Measure at 12 weeks

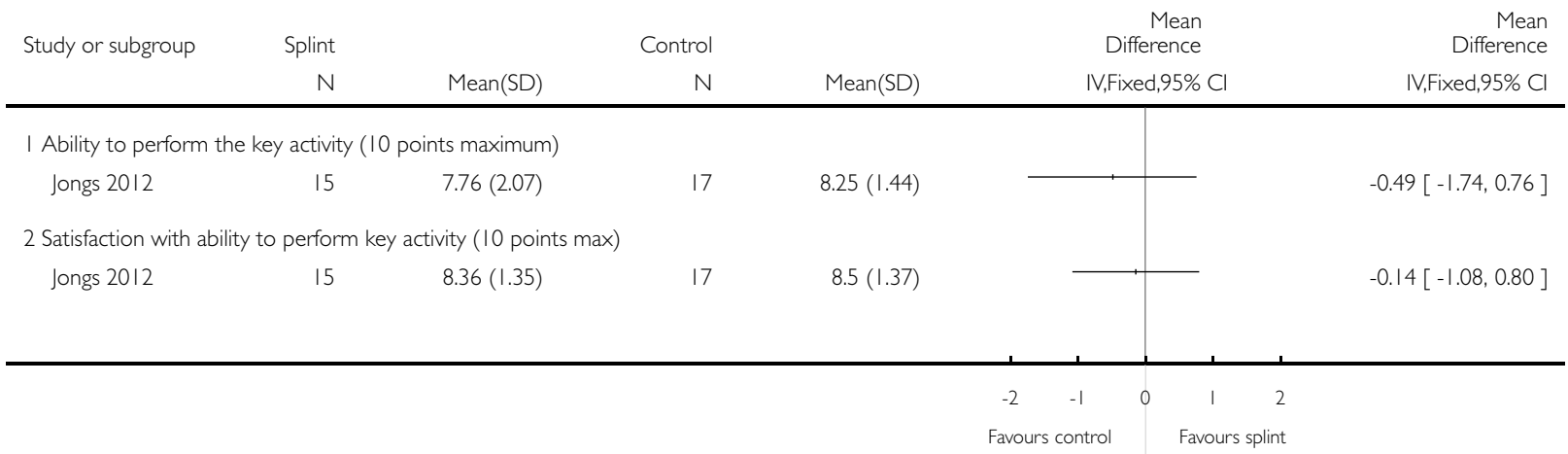


Analysis 15.3. Comparison I5 Dynamic wrist extension splint versus no intervention (control) (postimmobilisation), Outcome 3 Range of motion at 12 weeks.

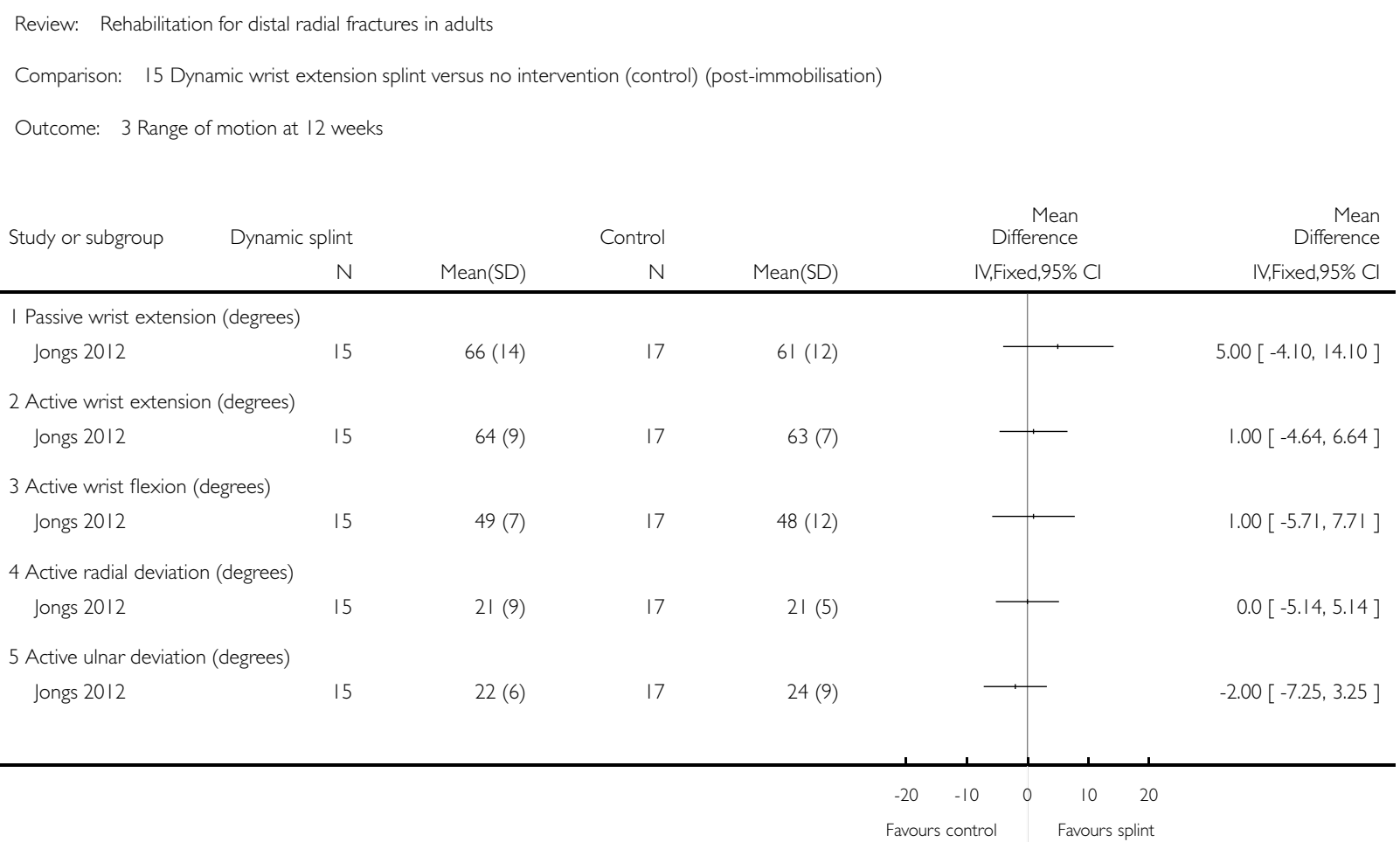

Analysis 16.1. Comparison 16 Post-immobilisation physiotherapy versus instructions from physician, Outcome I Wrist extension (degrees) at 6 weeks.

Review: Rehabilitation for distal radial fractures in adults

Comparison: 16 Post-immobilisation physiotherapy versus instructions from physician

Outcome: I Wrist extension (degrees) at 6 weeks

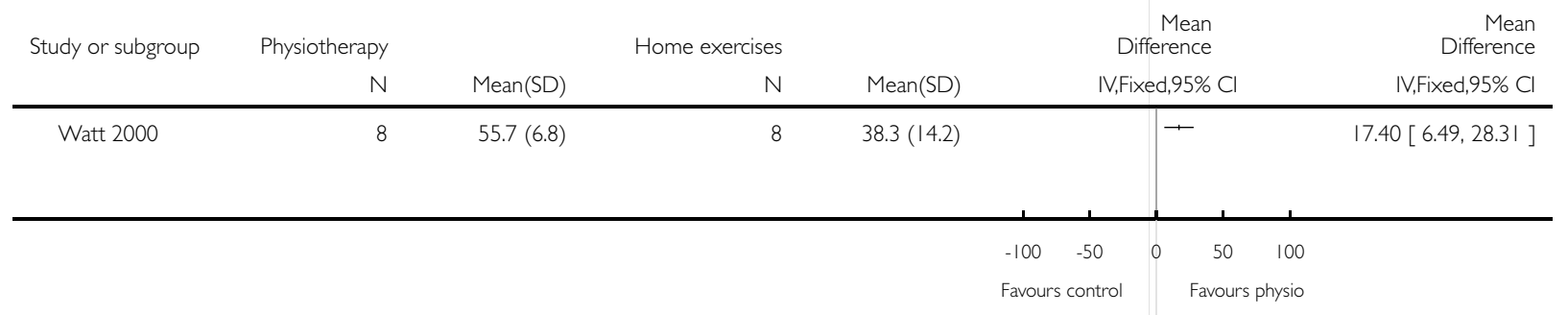

Rehabilitation for distal radial fractures in adults (Review)

Copyright $\odot 2015$ The Cochrane Collaboration. Published by John Wiley \& Sons, Ltd. 


\section{Analysis 17.I. Comparison 17 Pulsed electromagnetic field (PEMF) versus ice (post-immobilisation),}

Outcome I Pain and volume at day 5.

Review: Rehabilitation for distal radial fractures in adults

Comparison: 17 Pulsed electromagnetic field (PEMF) versus ice (post-immobilisation)

Outcome: I Pain and volume at day 5

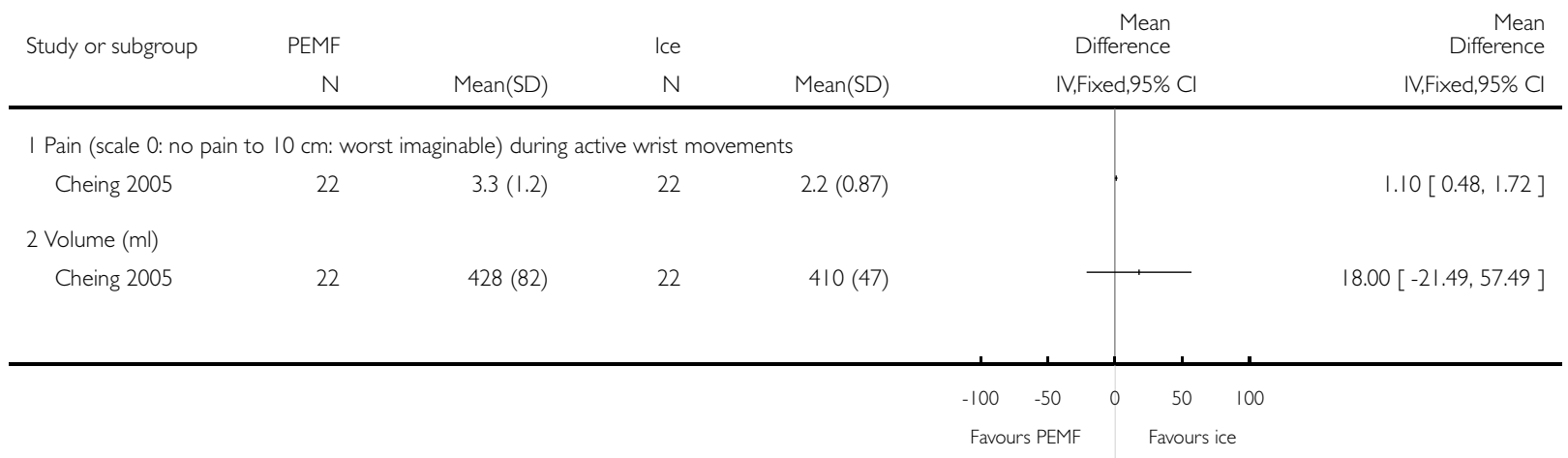


Analysis 17.2. Comparison I7 Pulsed electromagnetic field (PEMF) versus ice (post-immobilisation), Outcome 2 Range of motion at day 5.

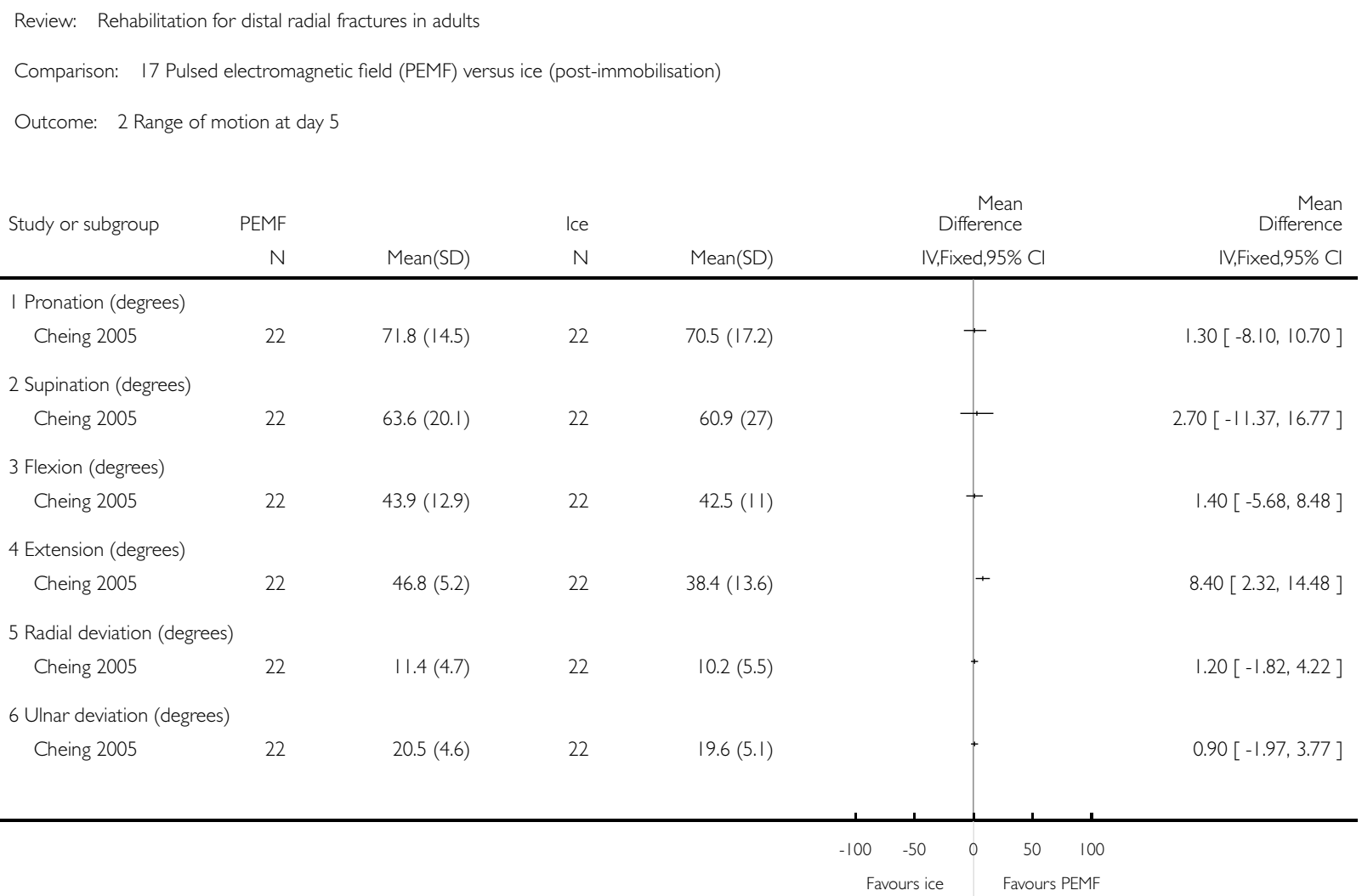


Analysis 18.I. Comparison 18 'Manual Edema Mobilization' (MEM) versus 'traditional' oedema treatment, Outcome I Canadian Occupational Performance Measure at 9 weeks (clinically important improvement).

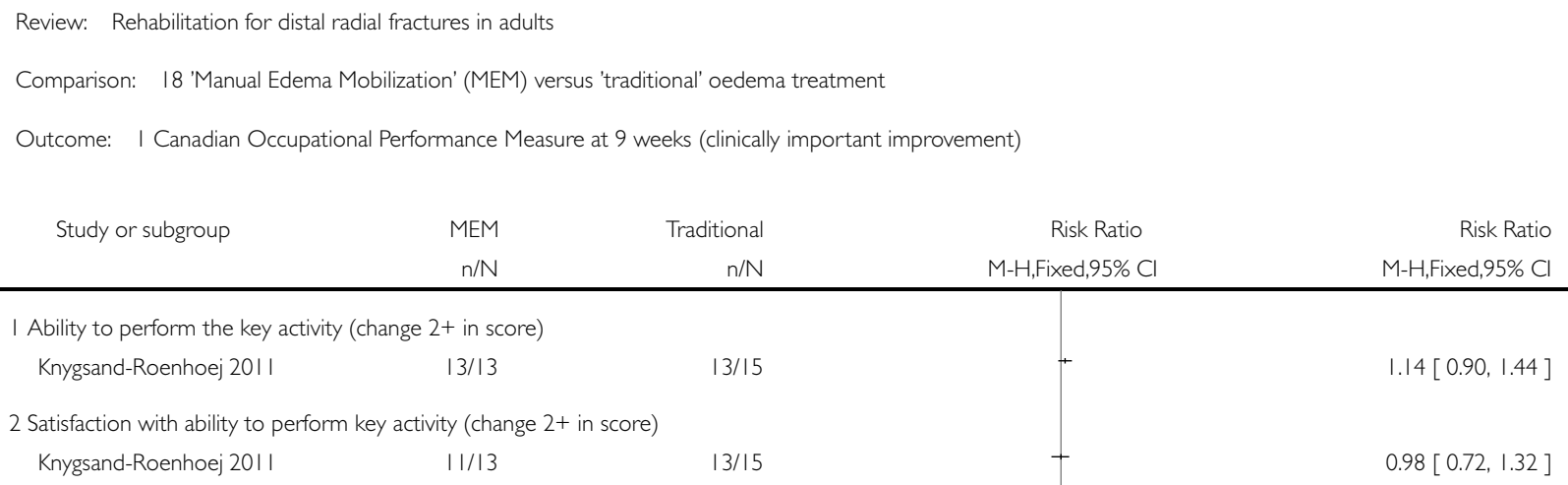

\section{Analysis 18.2. Comparison I8 'Manual Edema Mobilization' (MEM) versus 'traditional' oedema treatment, Outcome 2 Pain (VAS: 0 to 100: worst pain).}

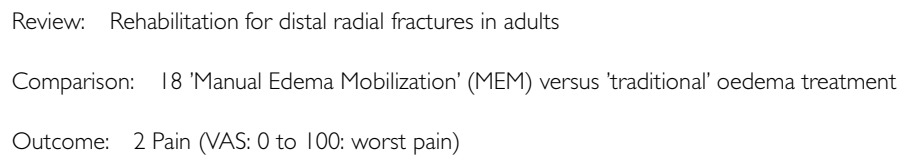

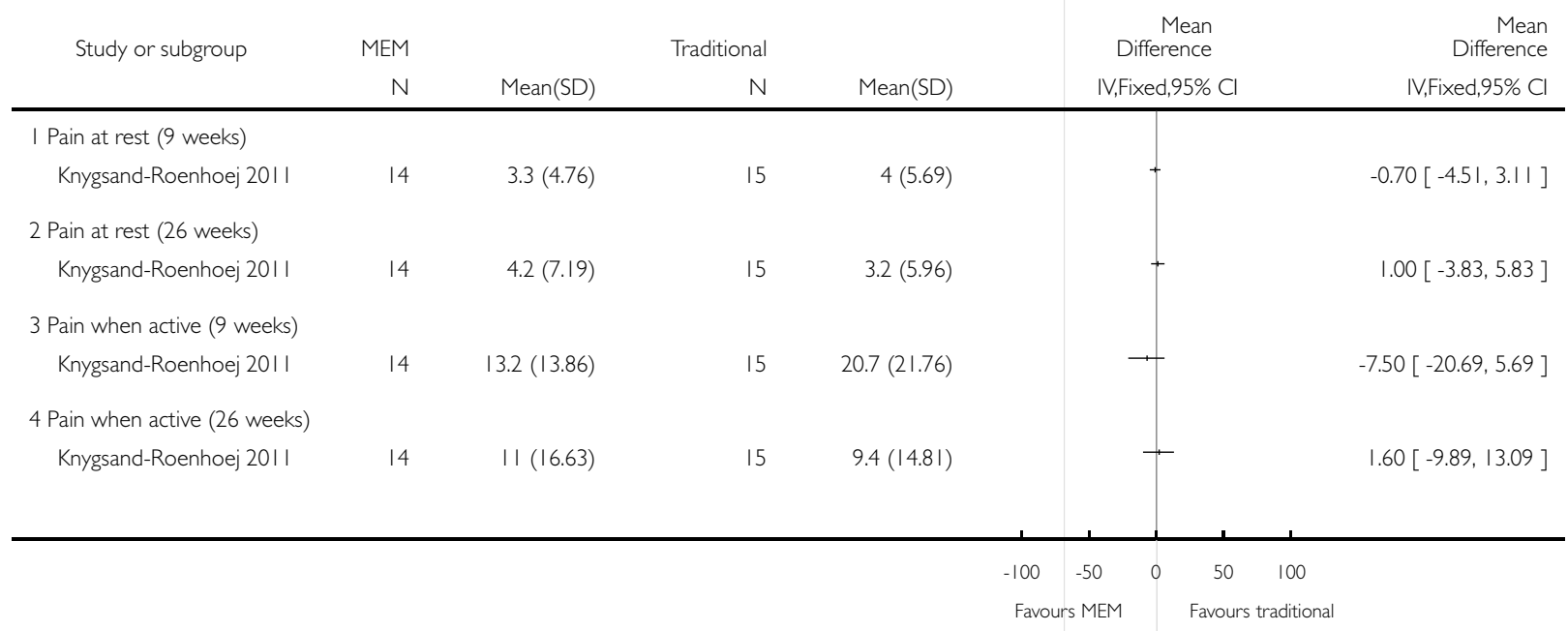




\section{Analysis 18.3. Comparison I8 'Manual Edema Mobilization' (MEM) versus 'traditional' oedema treatment,} Outcome 3 Complications.

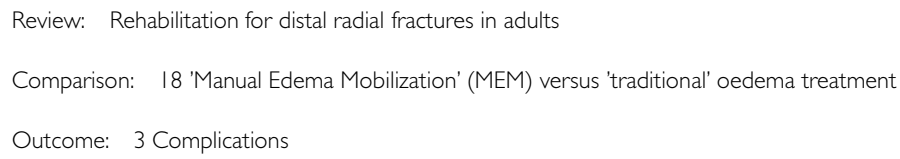

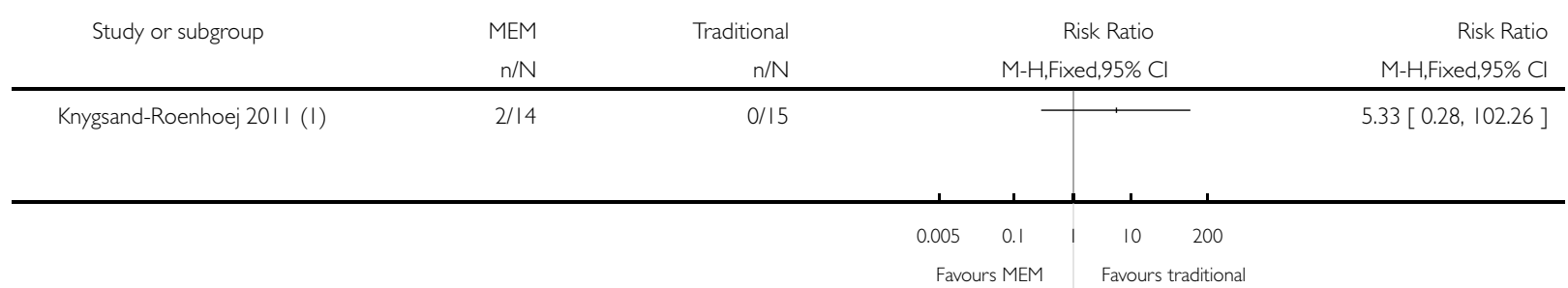

( I) I complex regional pain syndrome and I periarthrosis humeroscapularis

\section{Analysis 18.4. Comparison I8 'Manual Edema Mobilization' (MEM) versus 'traditional' oedema treatment,} Outcome 4 Number of occupational therapy sessions.

Review: Rehabilitation for distal radial fractures in adults

Comparison: 18 'Manual Edema Mobilization' (MEM) versus 'traditional' oedema treatment

Outcome: 4 Number of occupational therapy sessions

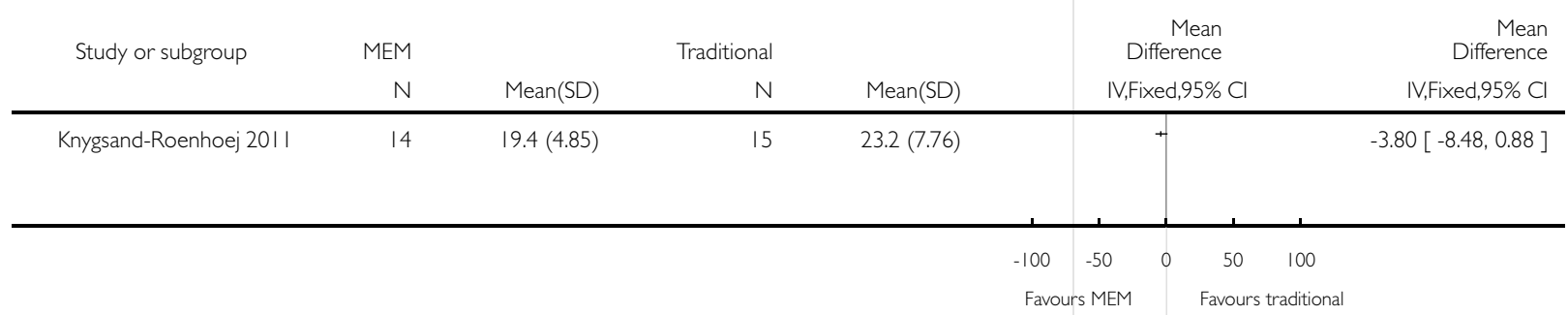


Analysis 18.5. Comparison I8 'Manual Edema Mobilization' (MEM) versus 'traditional' oedema treatment, Outcome 5 Receiving oedema treatment after scheduled period.

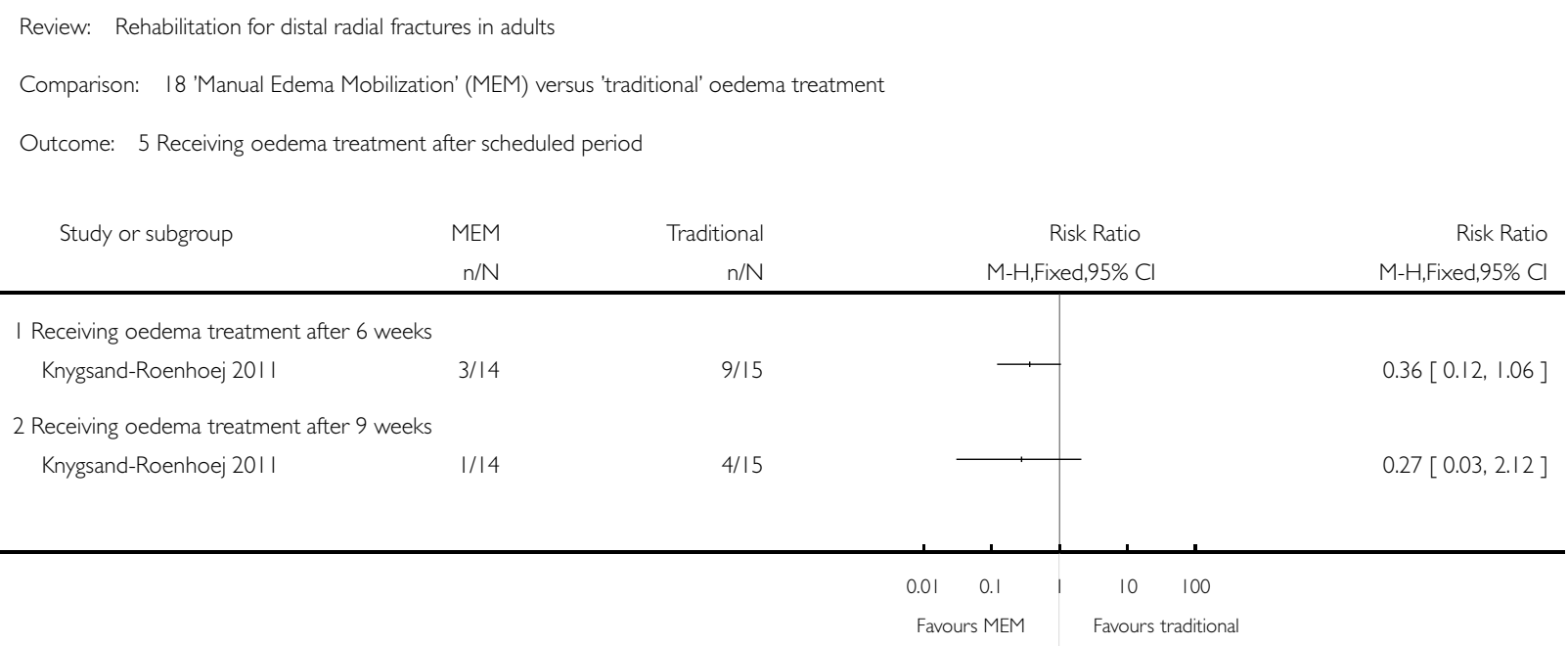

Analysis 18.6. Comparison 18 'Manual Edema Mobilization' (MEM) versus 'traditional' oedema treatment, Outcome 6 Oedema: volume difference between injured and non-injured side $(\mathrm{mL})$.

Review: Rehabilitation for distal radial fractures in adults

Comparison: 18 'Manual Edema Mobilization' (MEM) versus 'traditional' oedema treatment

Outcome: 6 Oedema: volume difference between injured and non-injured side $(\mathrm{mL})$

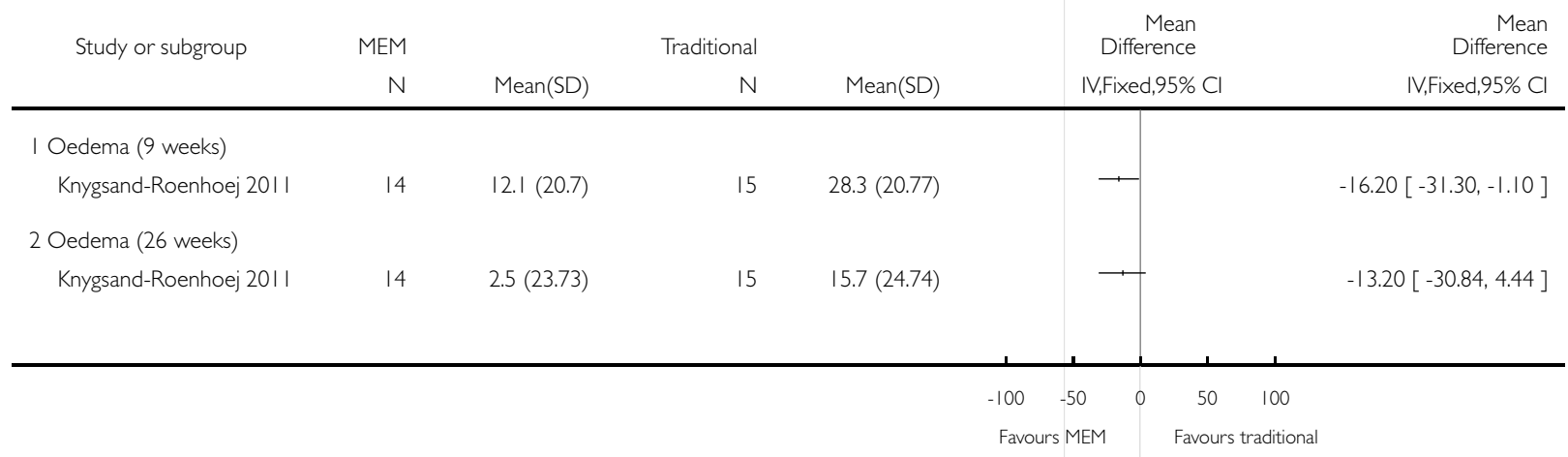


Analysis 19.1. Comparison 19 Supervised training by physiotherapist versus instructions by physician (from definitive treatment), Outcome I Strength and power at 12 weeks.

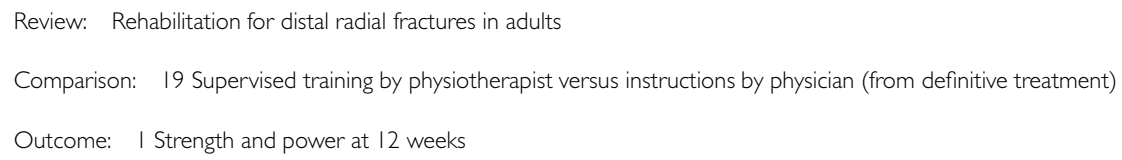

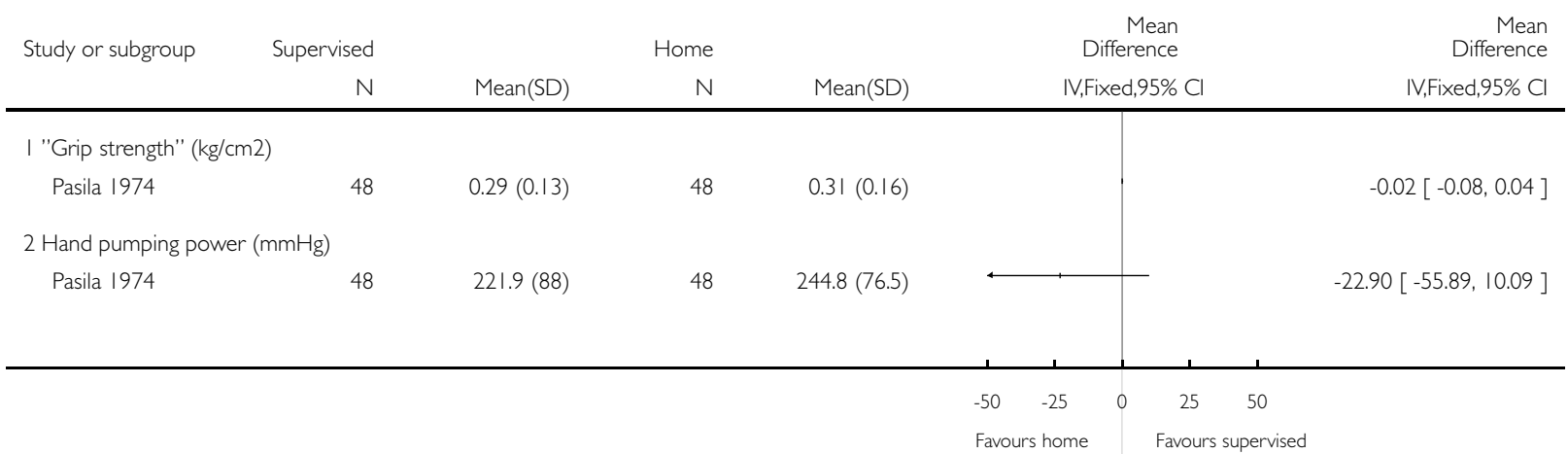


Analysis 19.2. Comparison 19 Supervised training by physiotherapist versus instructions by physician (from definitive treatment), Outcome 2 Range of motion at 12 weeks.

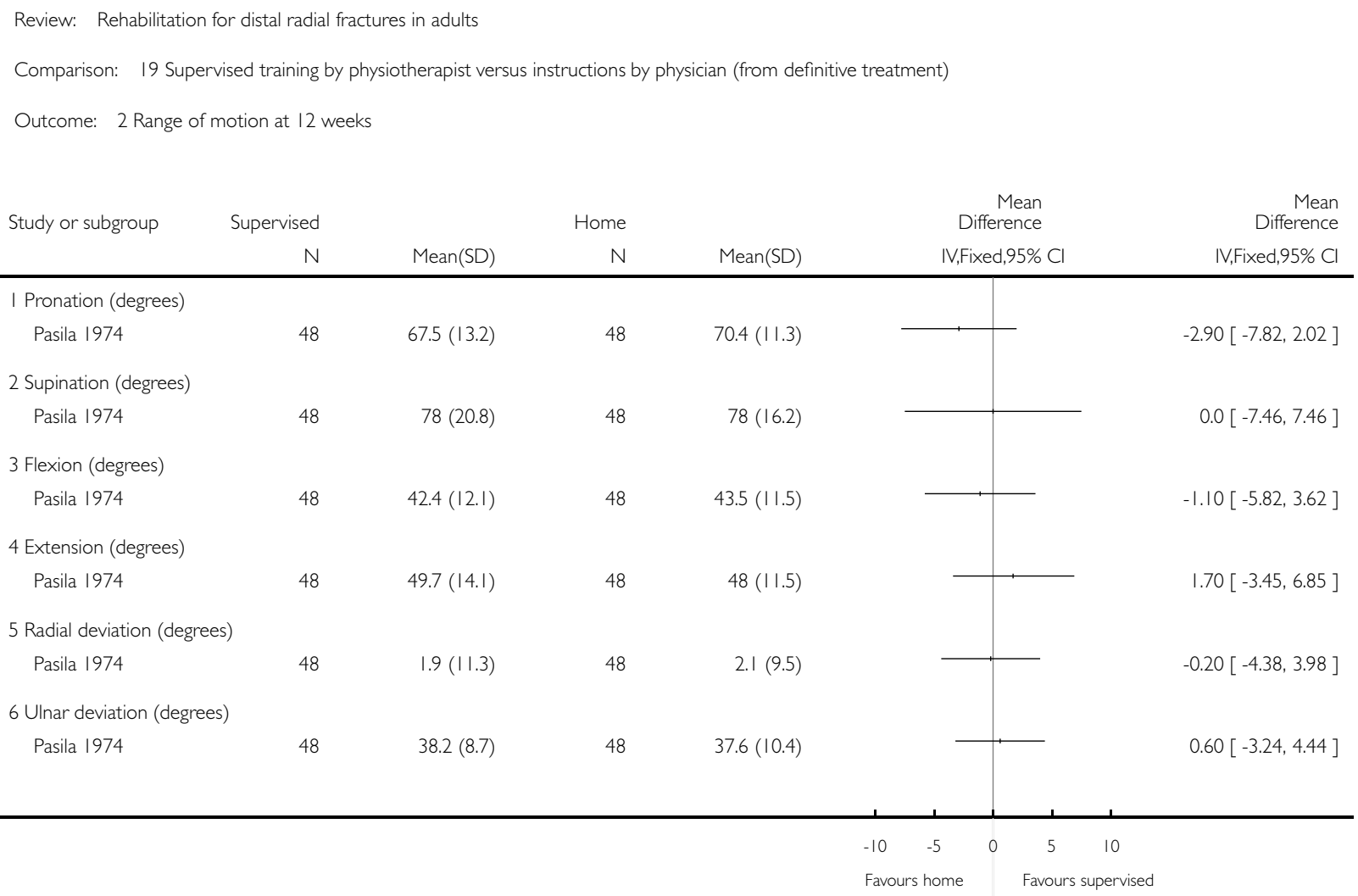


Analysis 20.1. Comparison 20 Physiotherapy/occupational therapy versus home exercise programme (postsurgery), Outcome I PRWE scores at 6 weeks (0: normal to I50: worst outcome).

Review: Rehabilitation for distal radial fractures in adults

Comparison: 20 Physiotherapy/occupational therapy versus home exercise programme (post-surgery)

Outcome: I PRWE scores at 6 weeks (0: normal to 150: worst outcome)

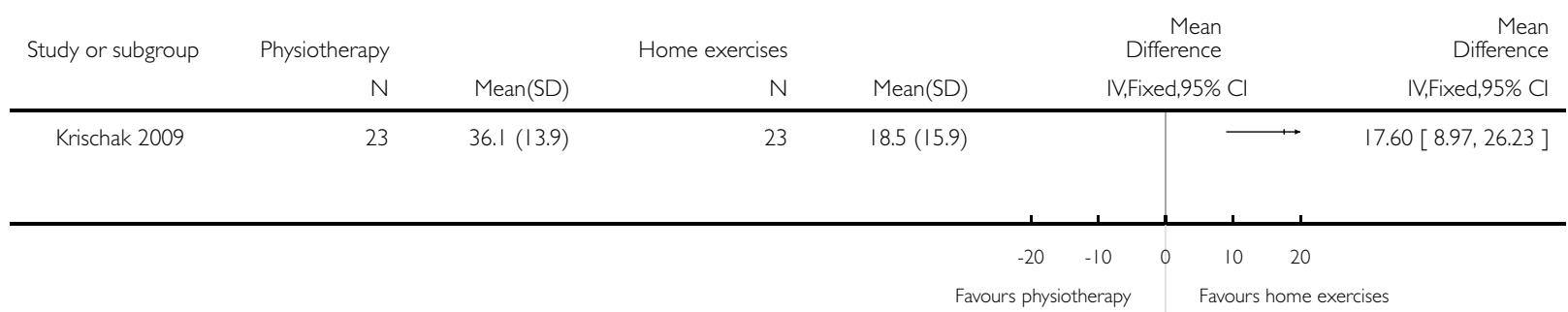

Analysis 20.2. Comparison 20 Physiotherapy/occupational therapy versus home exercise programme (postsurgery), Outcome 2 DASH score (0 to 100: higher scores = worse upper-extremity function).

Review: Rehabilitation for distal radial fractures in adults

Comparison: 20 Physiotherapy/occupational therapy versus home exercise programme (post-surgery)

Outcome: 2 DASH score (0 to 100: higher scores = worse upper-extremity function)

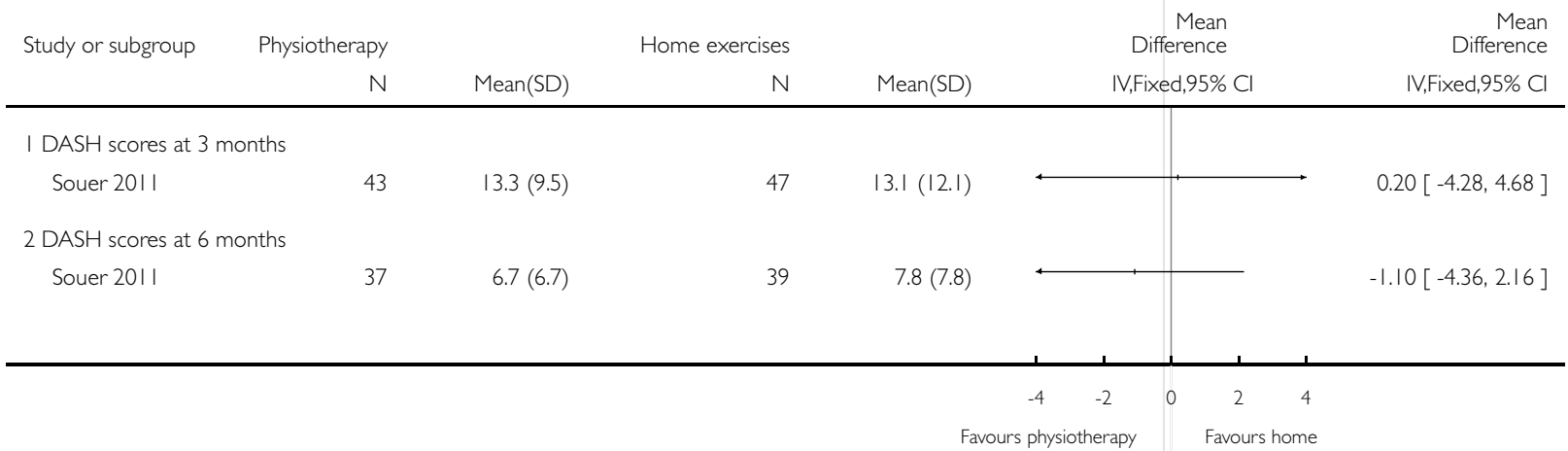


Analysis 20.3. Comparison 20 Physiotherapy/occupational therapy versus home exercise programme (postsurgery), Outcome 3 Mayo wrist score (0 to 100: higher scores = best functional outcome).

Review: Rehabilitation for distal radial fractures in adults

Comparison: 20 Physiotherapy/occupational therapy versus home exercise programme (post-surgery)

Outcome: 3 Mayo wrist score (0 to 100: higher scores = best functional outcome)

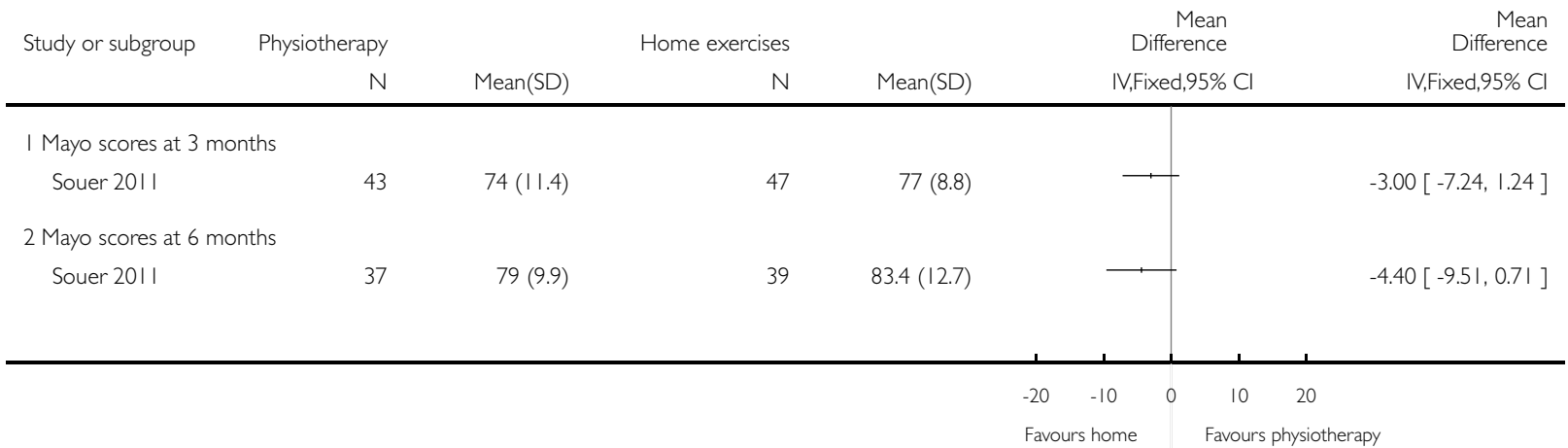

Analysis 20.4. Comparison 20 Physiotherapy/occupational therapy versus home exercise programme (postsurgery), Outcome 4 Pain at rest (VAS: 0 to 10: worst pain).

Review: Rehabilitation for distal radial fractures in adults

Comparison: 20 Physiotherapy/occupational therapy versus home exercise programme (post-surgery)

Outcome: 4 Pain at rest (VAS: 0 to I0: worst pain)

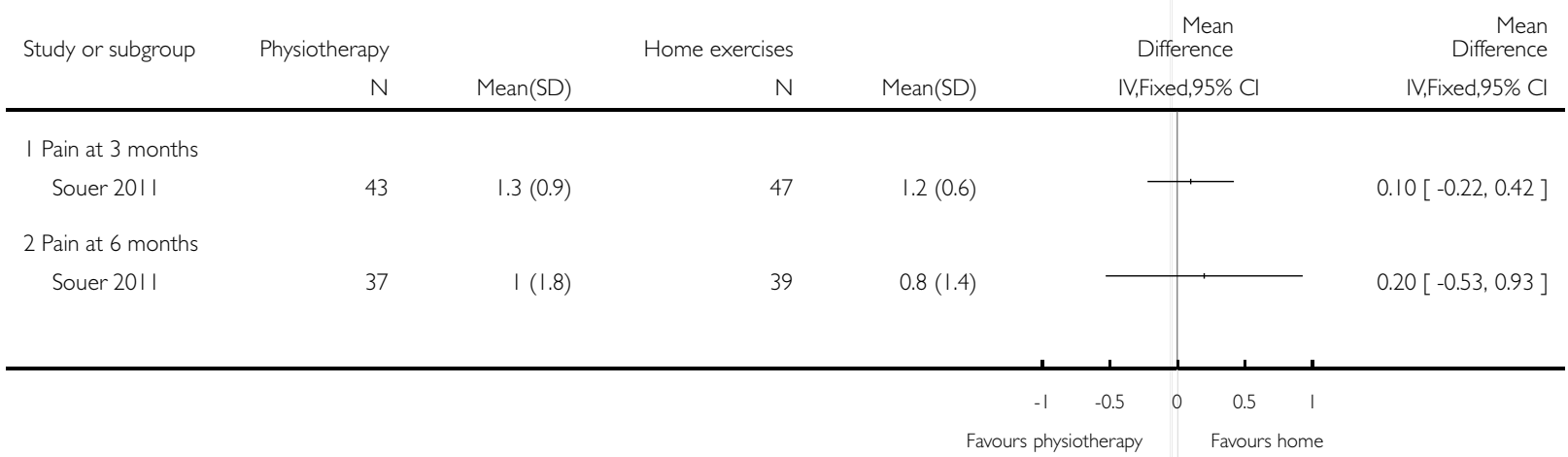


Analysis 20.5. Comparison 20 Physiotherapy/occupational therapy versus home exercise programme (postsurgery), Outcome 5 Grip strength (kg).

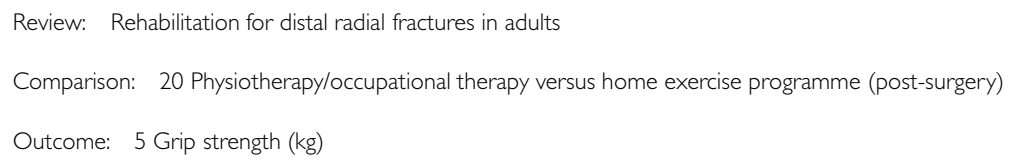

\begin{tabular}{|c|c|c|c|c|c|c|}
\hline \multirow[t]{2}{*}{ Study or subgroup } & Physiotherapy & \multicolumn{3}{|c|}{ Home exercises } & $\begin{array}{r}\text { Mean } \\
\text { Difference }\end{array}$ & \multirow{2}{*}{$\begin{array}{r}\text { Mean } \\
\text { Difference } \\
\text { IV,Fixed,95\% Cl }\end{array}$} \\
\hline & $\mathrm{N}$ & Mean(SD) & N & Mean(SD) & IV,Fixed,95\% Cl & \\
\hline \multicolumn{7}{|c|}{ I Grip strength at 3 months } \\
\hline Souer 2011 & 43 & $20(7.8)$ & 47 & $24.8(10.2)$ & $一$ & $-4.80[-8.53,-1.07]$ \\
\hline \multicolumn{7}{|c|}{2 Grip strength at 6 months } \\
\hline Souer 2011 & 37 & $23(8.1)$ & 39 & $25.7(8.3)$ & $\longrightarrow$ & $-2.70[-6.39,0.99]$ \\
\hline
\end{tabular}

Analysis 20.6. Comparison 20 Physiotherapy/occupational therapy versus home exercise programme (postsurgery), Outcome 6 Pinch strength (kg).

Review: Rehabilitation for distal radial fractures in adults

Comparison: 20 Physiotherapy/occupational therapy versus home exercise programme (post-surgery)

Outcome: 6 Pinch strength $(\mathrm{kg})$

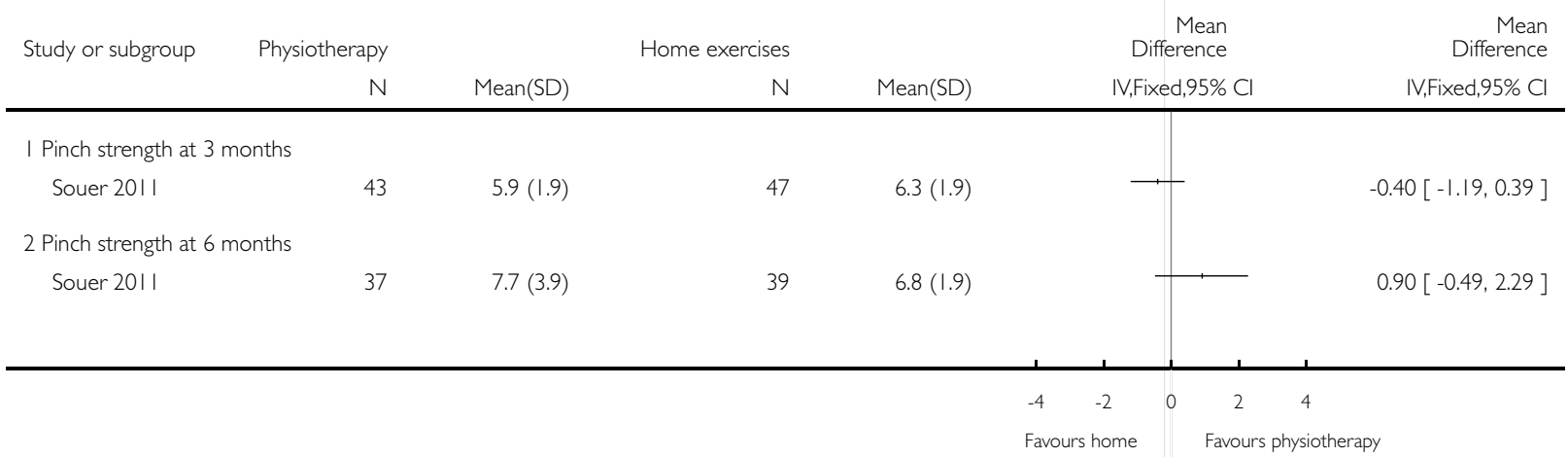


Analysis 20.7. Comparison 20 Physiotherapy/occupational therapy versus home exercise programme (postsurgery), Outcome 7 Range of motion at 3 months.

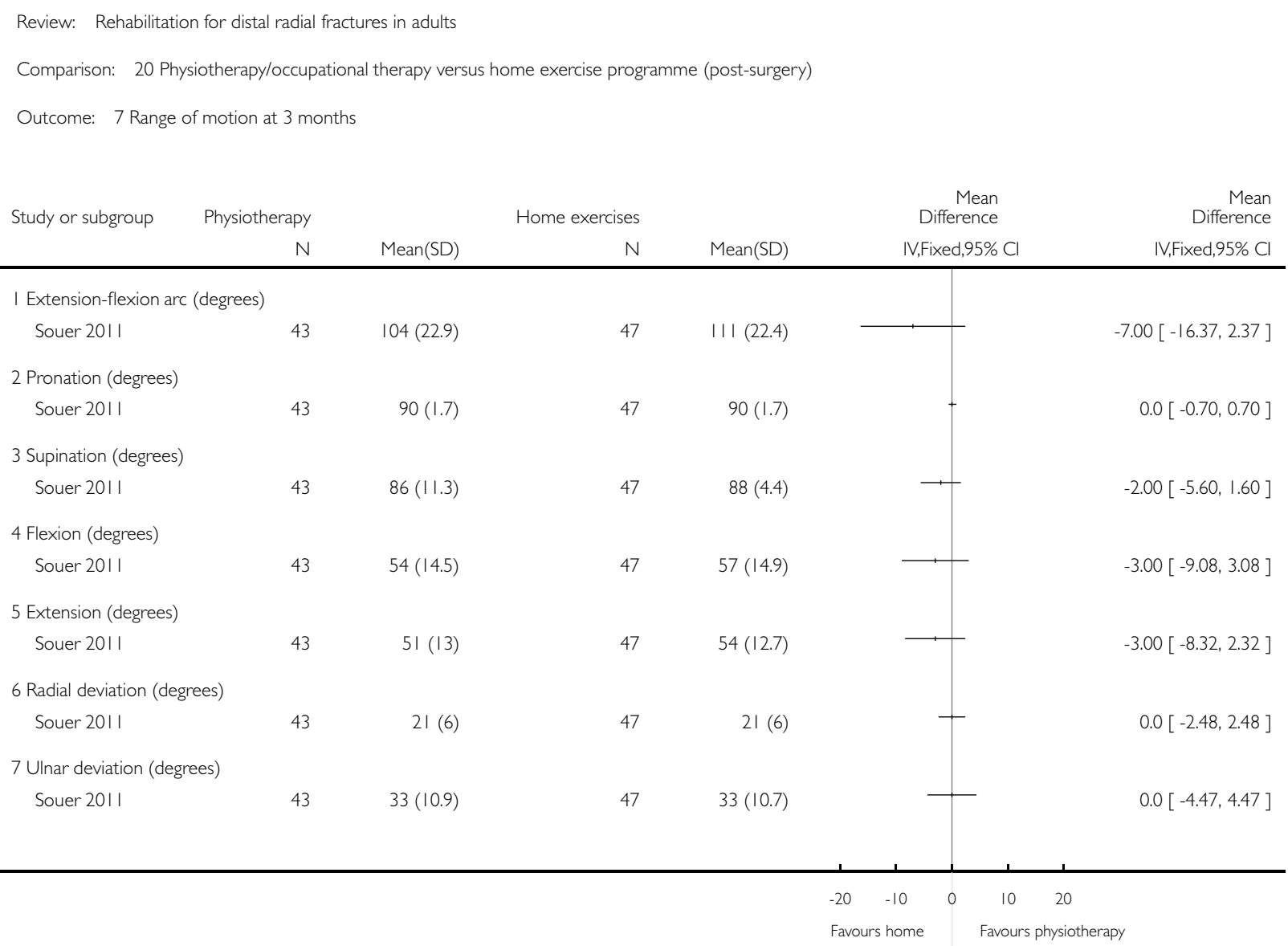


Analysis 20.8. Comparison 20 Physiotherapy/occupational therapy versus home exercise programme (postsurgery), Outcome 8 Range of motion at 6 months.

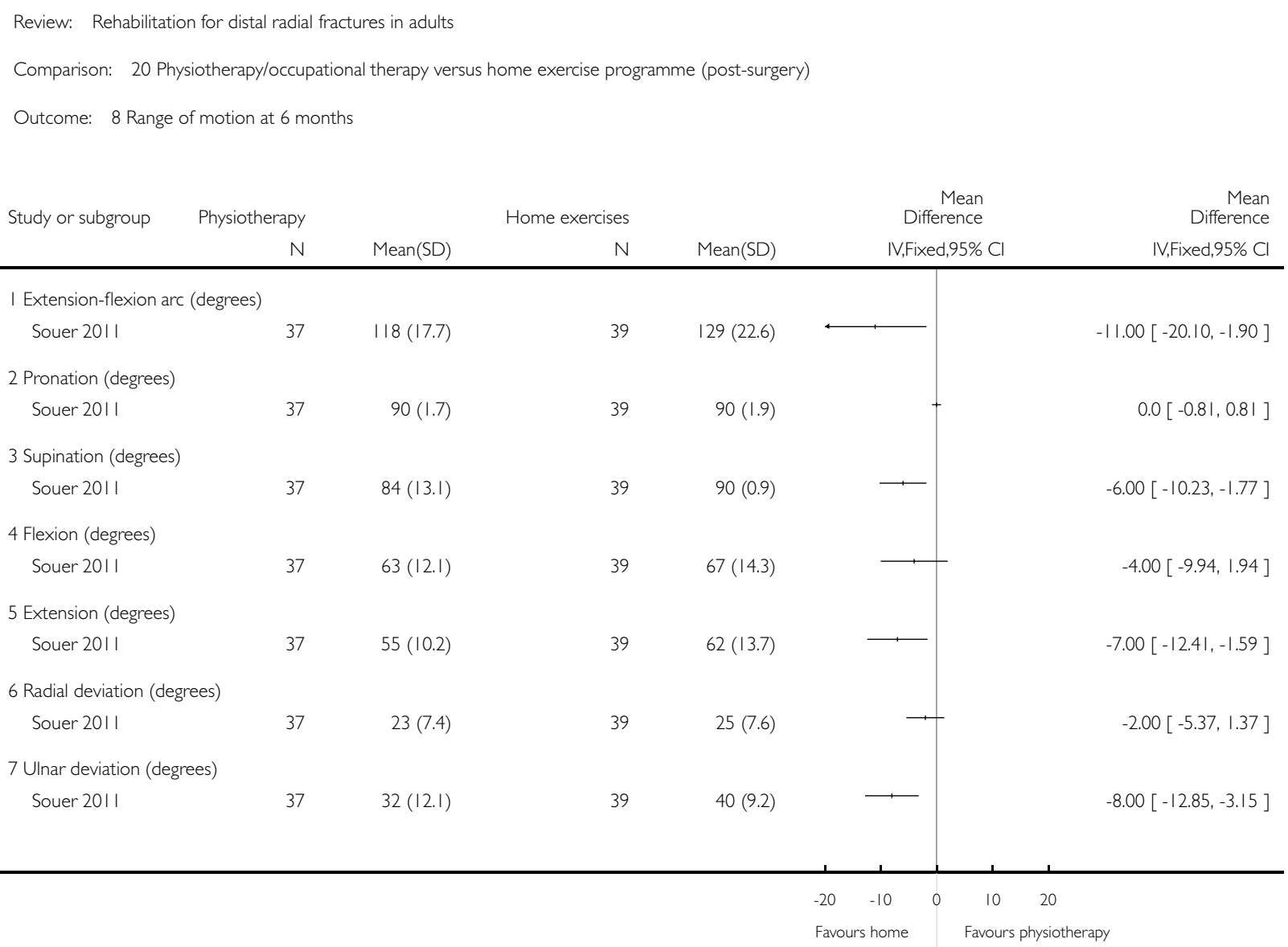


Analysis 20.9. Comparison 20 Physiotherapy/occupational therapy versus home exercise programme (postsurgery), Outcome 9 Complications.

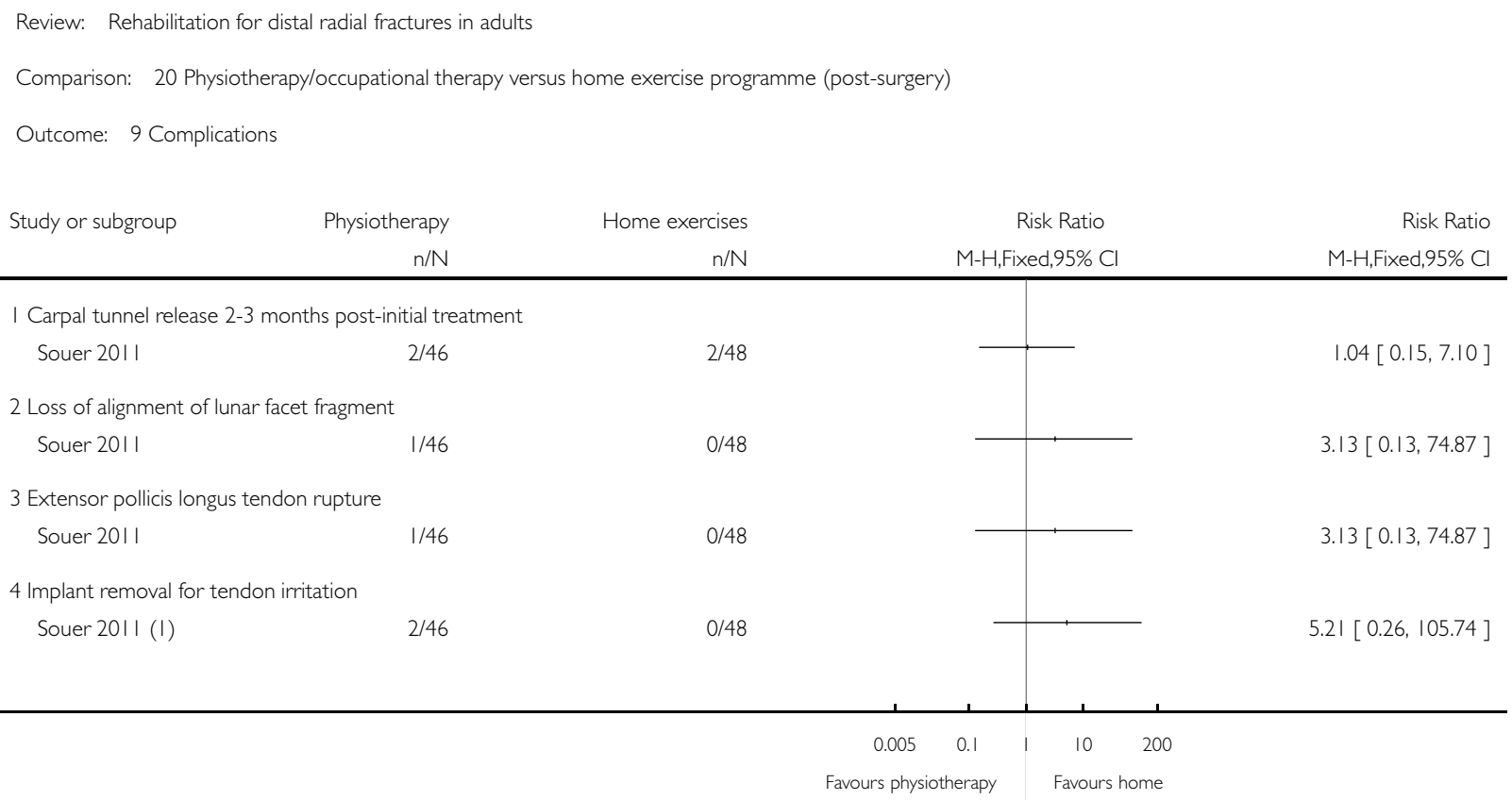

(I) Deduced that 2 of the 3 reported cases occurred $<6$ months 
Analysis 2I.I. Comparison 2 I Accelerated (start 2 weeks) versus usual (start 6 weeks) rehabilitation postsurgery, Outcome I DASH score (0 to 100: higher scores = worse upper-extremity function).

Review: Rehabilitation for distal radial fractures in adults

Comparison: 21 Accelerated (start 2 weeks) versus usual (start 6 weeks) rehabilitation post-surgery

Outcome: I DASH score (0 to I00: higher scores = worse upper-extremity function)

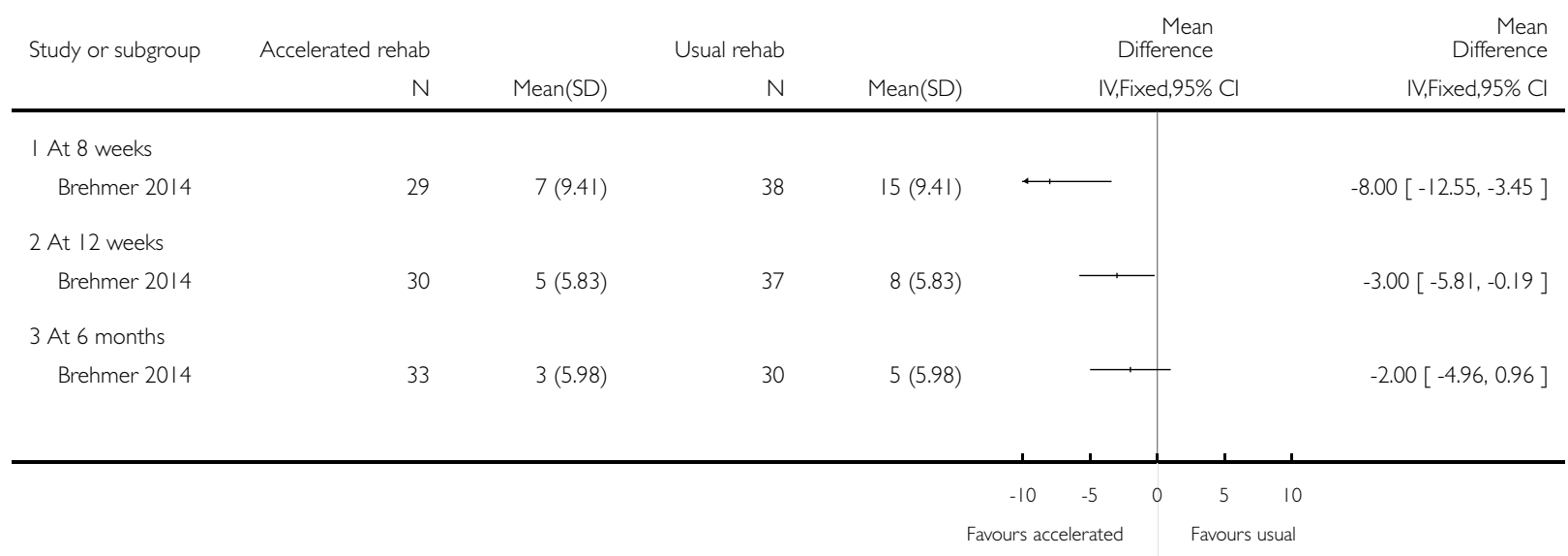

Analysis 21.2. Comparison 21 Accelerated (start 2 weeks) versus usual (start 6 weeks) rehabilitation postsurgery, Outcome 2 Grip strength (lb).

Review: Rehabilitation for distal radial fractures in adults

Comparison: 21 Accelerated (start 2 weeks) versus usual (start 6 weeks) rehabilitation post-surgery

Outcome: 2 Grip strength (lb)

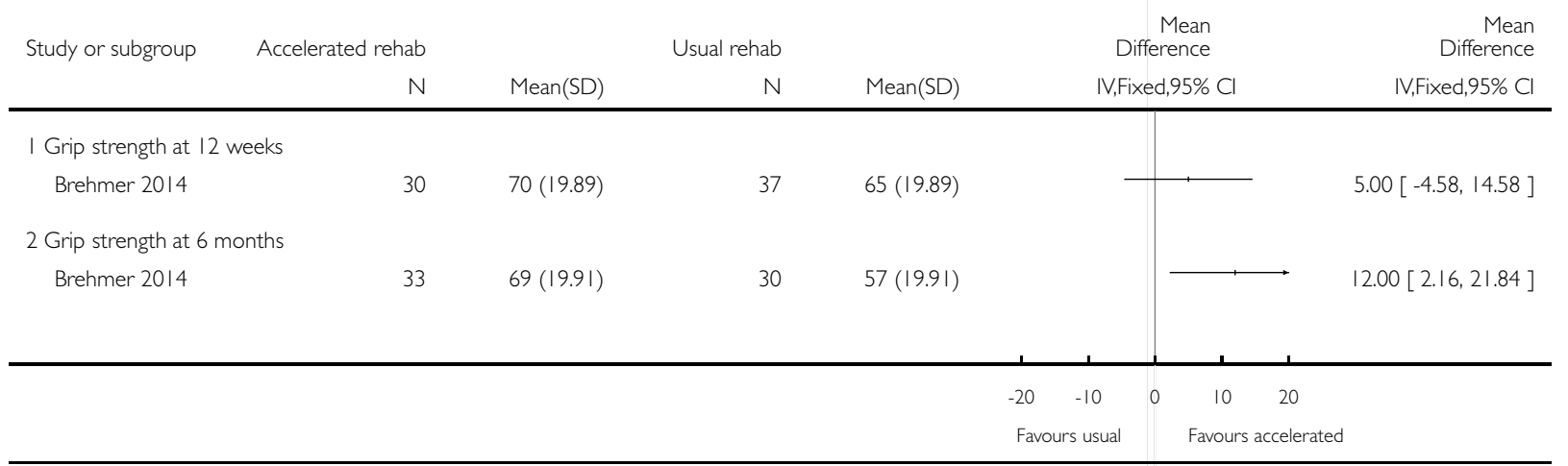

Rehabilitation for distal radial fractures in adults (Review)

Copyright $\odot 2015$ The Cochrane Collaboration. Published by John Wiley \& Sons, Ltd. 
Analysis 21.3. Comparison 21 Accelerated (start 2 weeks) versus usual (start 6 weeks) rehabilitation postsurgery, Outcome 3 Pinch strength (lb).

Review: Rehabilitation for distal radial fractures in adults

Comparison: 21 Accelerated (start 2 weeks) versus usual (start 6 weeks) rehabilitation post-surgery

Outcome: 3 Pinch strength (lb)

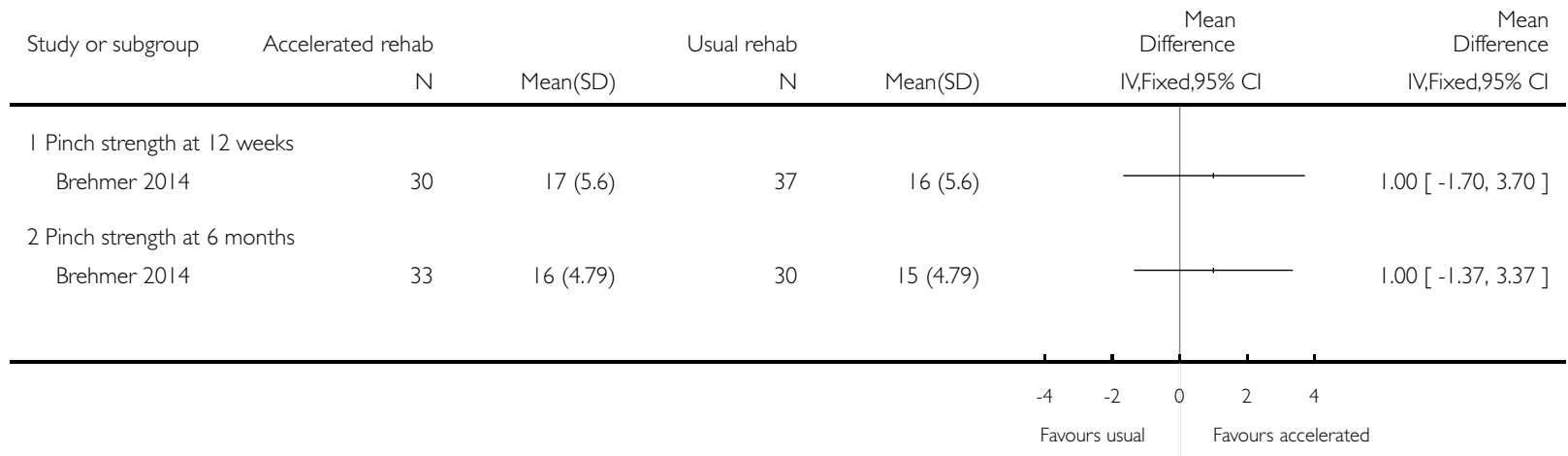


Analysis 21.4. Comparison 21 Accelerated (start 2 weeks) versus usual (start 6 weeks) rehabilitation postsurgery, Outcome 4 Range of motion at 12 weeks.

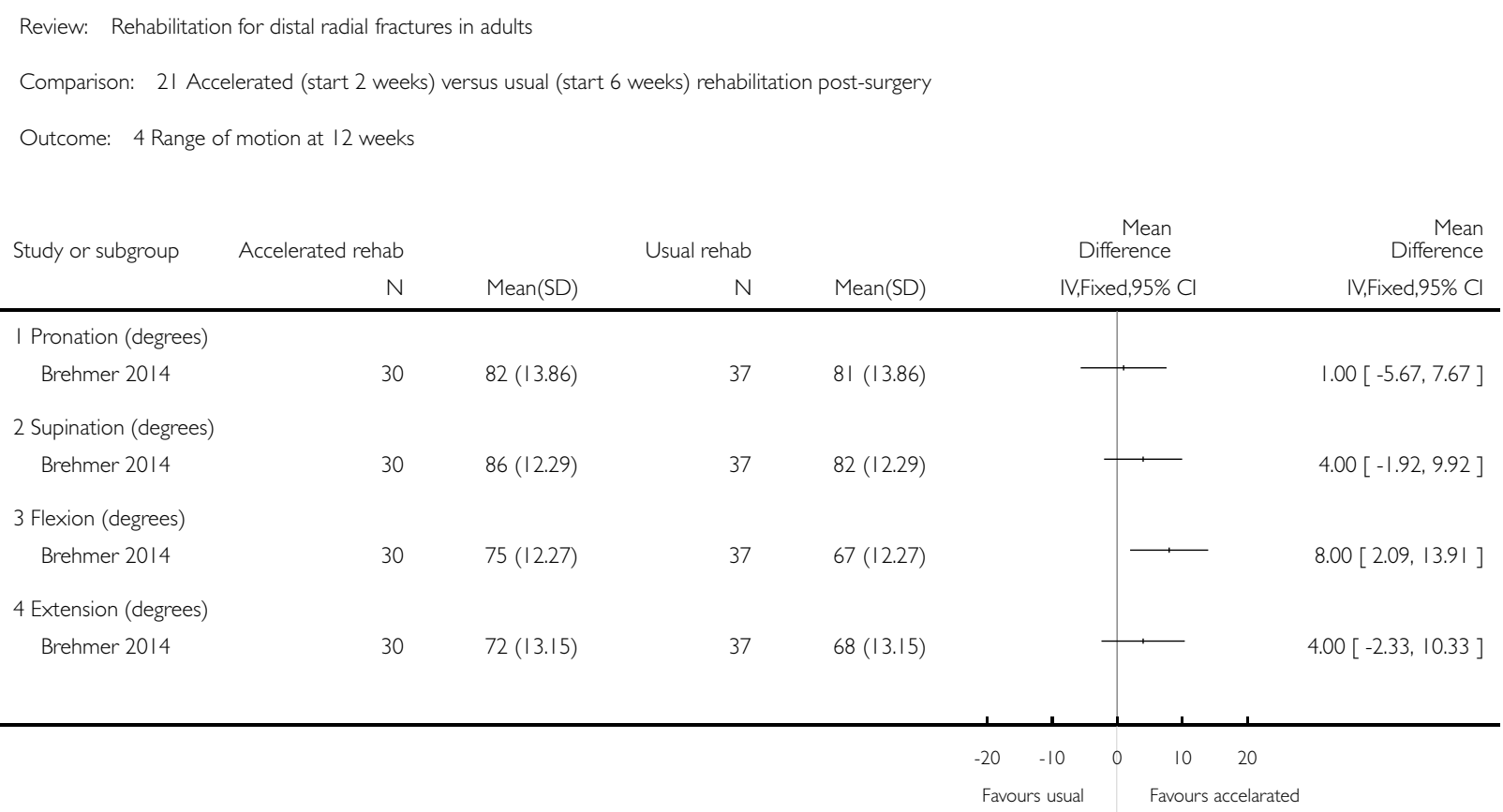


Analysis 21.5. Comparison 2 I Accelerated (start 2 weeks) versus usual (start 6 weeks) rehabilitation postsurgery, Outcome 5 Range of motion at 6 months.

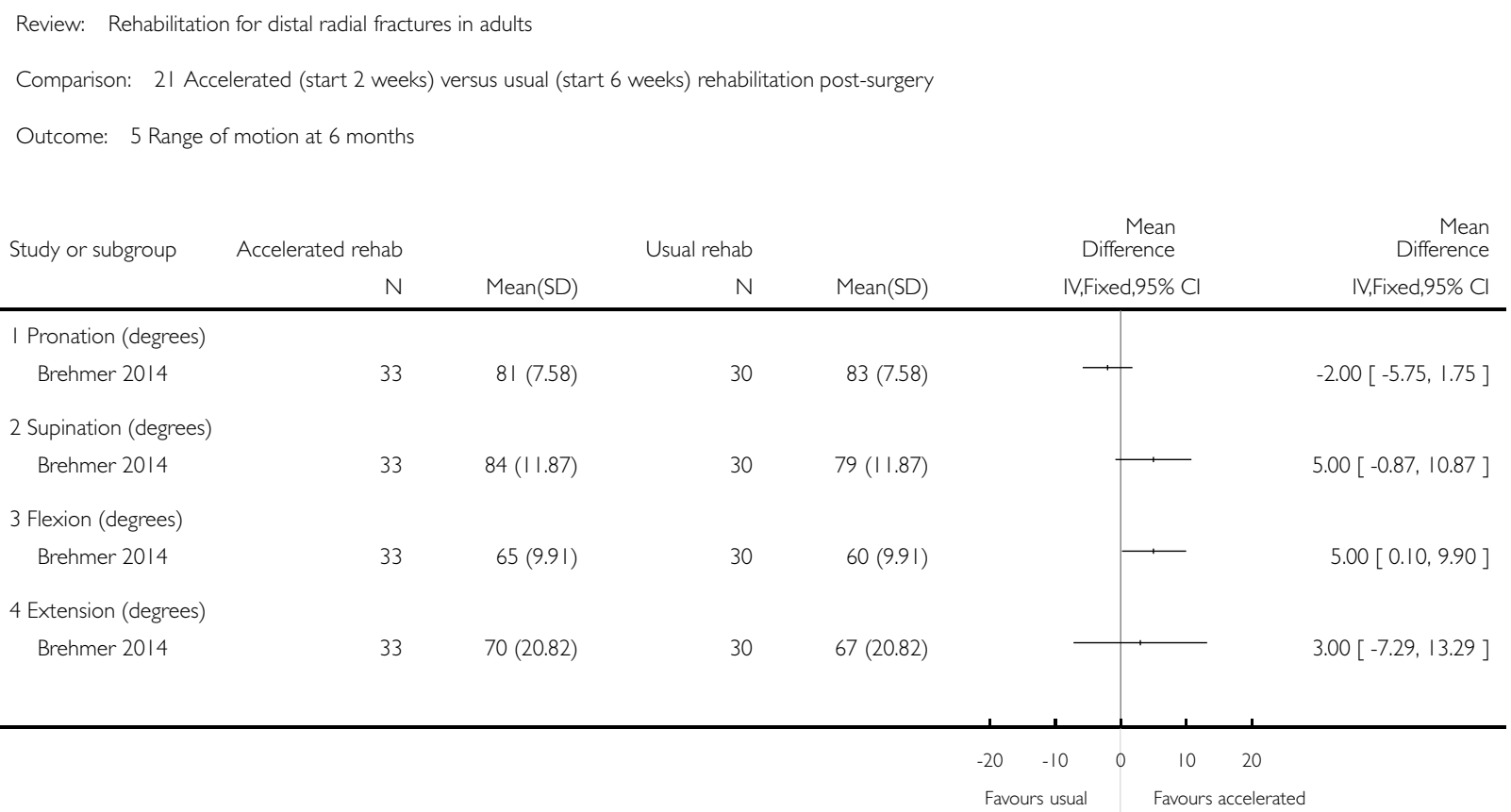




\section{Analysis 21.6. Comparison 21 Accelerated (start 2 weeks) versus usual (start 6 weeks) rehabilitation post- surgery, Outcome 6 Complications.}

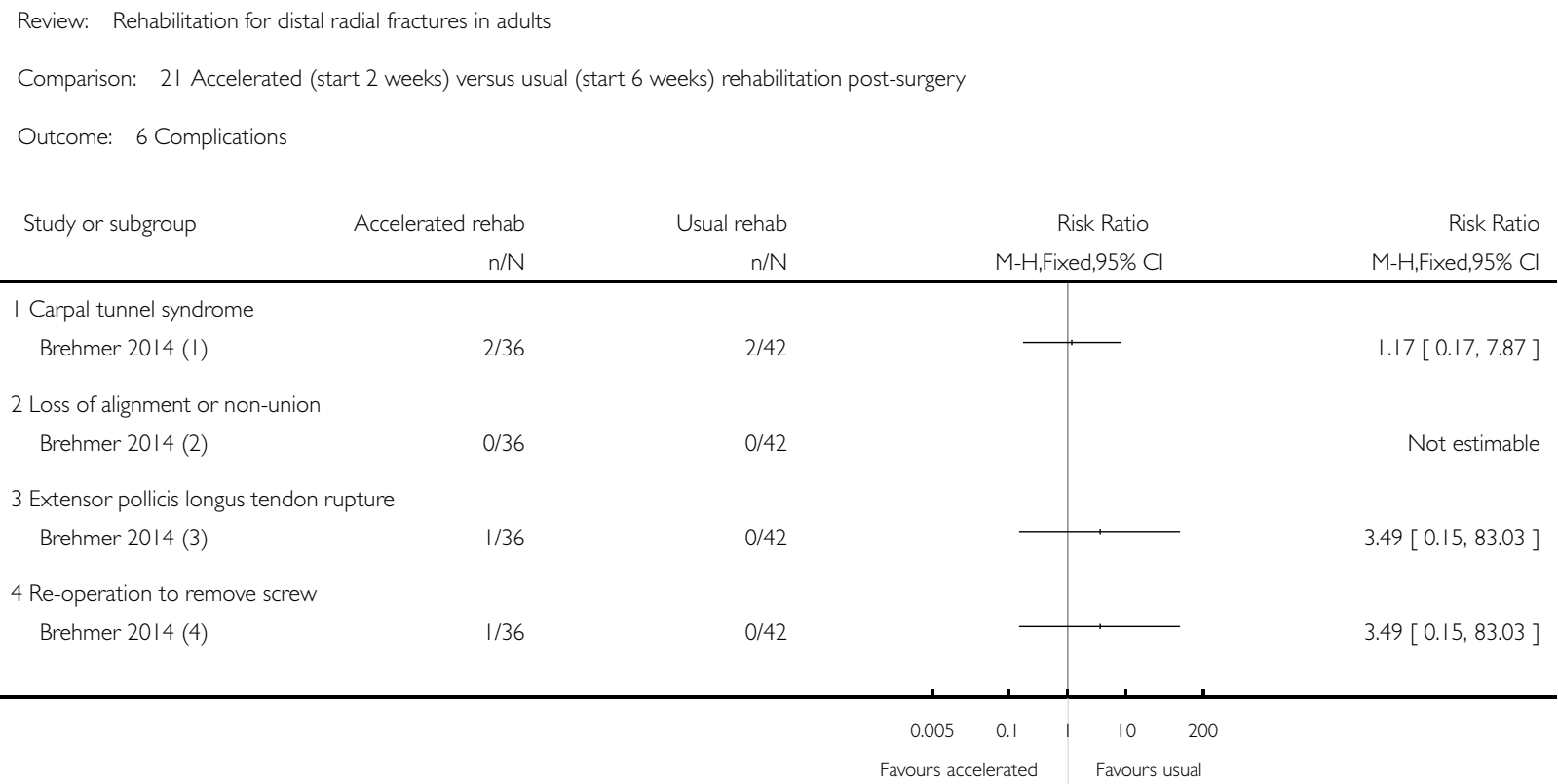

( I) All 4 cases resolved spontaneously

(2) There was no loss in fracture alignment

(3) Occurred I month post-op; underwent tendon transfer surgery at 3 months

(4) Screw protruding in the distal radioulnar joint removed at 7 weeks

\section{ADDITIONAL TABLES}

Table 1. Descriptions and putative mechanisms of some rehabilitation interventions

\begin{tabular}{|c|c|c|c|}
\hline Intervention & Description & Aim & Putative mechanism \\
\hline Advice and instructions & $\begin{array}{l}\text { These usually include patient } \\
\text { education on the ways to cope } \\
\text { with the consequences of the in- } \\
\text { jury (such as pain) and avoid } \\
\text { some of the common prob- } \\
\text { lems, such as stiff joints, asso- } \\
\text { ciated with immobilisation and } \\
\text { instruction on exercising un- } \\
\text { involved joints during immo- } \\
\text { bilisation, with the wrist joint } \\
\text { added subsequently } \\
\text { These are delivered in various }\end{array}$ & $\begin{array}{l}\text { To empower the patient in their } \\
\text { own recovery }\end{array}$ & $\begin{array}{l}\text { Facilitating patient participa- } \\
\text { tion in their own recovery by } \\
\text { providing information on the } \\
\text { means. One key intervention is } \\
\text { joint mobilisation (see below) }\end{array}$ \\
\hline
\end{tabular}


ways

\begin{tabular}{|c|c|c|c|}
\hline Cross-education & $\begin{array}{l}\text { This involves strength training } \\
\text { (exercises) of the opposite non- } \\
\text { injured hand (cross-education) }\end{array}$ & $\begin{array}{l}\text { To improve function of the in- } \\
\text { volved hand }\end{array}$ & $\begin{array}{l}\text { Strength training in one limb } \\
\text { produces neural adaptations } \\
\text { that increase strength in the } \\
\text { contralateral limb }\end{array}$ \\
\hline
\end{tabular}

Dynamic wrist splint

\begin{tabular}{l} 
Ice \\
\hline Joint mobilisation \\
(active and passive)
\end{tabular}

Joint mobilisation

(a) A splint applied to the fore- To improve hand function by
arm that provides a continuous increasing range of wrist mo-
low-load stretch while enabling tion

hand movement

Ice or iced water or other cooling devices
To relieve pain and reduce swelling

Stretching the wrist in a steady way will improve physiological parameters such as blood flow to the muscles

Ice reduces metabolic activity within the tissues and thus should prevent secondary damage. It also reduces pain signals to the central nervous system

This involves the movement of a joint and can take two basic forms, which are not mutually exclusive:

Active: under the control of the patient's own musculature Passive: via an externally applied force, such as by a therapist

Passive mobilisation can also be applied by machines that repeatedly move the joint(s) through a prespecified range of motion

To restore or maintain range of Movement is part of keeping acmovement and avoid complica- tive and fit. Not moving results tions in loss of mobility (stiffness and joint contracture) and reduced muscle strength

The range of movement under active control is usually less than that available passively; but a key advantage is that active movement emulates physiological function

Passive mobilisation may be undertaken by the patient, but is more usually applied by a therapist

Automation of the intervention via a machine can free up clinician time but involves additional technology and restricts the patient to a specific location

Soft tissue compression (cyclic or intermittent)
This is delivered in various ways, generally involving machines providing an intermittent pneumatic compression delivered via an inflatable device round the hand or wrist
To reduce or prevent swelling

Pulsed compression of surrounding tissues helps in circulation and prevents build up of fluid that would impair movements and recovery 
Table 2. Summary of study populations

\begin{tabular}{|c|c|c|c|c|c|c|c|}
\hline Study ID & Number & $\begin{array}{l}\text { Gender \& age } \\
\text { (years) }\end{array}$ & $\begin{array}{l}\text { Fracture } \\
(\mathrm{Ex}=\text { exclu- } \\
\text { sion })\end{array}$ & $\begin{array}{l}\text { Definitive } \\
\text { treatment } \\
\text { and timing }\end{array}$ & $\begin{array}{l}\text { Complica- } \\
\text { tions } \\
(\mathrm{Ex}=\text { exclu- } \\
\text { sion })\end{array}$ & $\begin{array}{l}\text { Comorbidi- } \\
\text { ties or } \\
\text { prior function } \\
(\text { Ex = exclu- } \\
\text { sion })\end{array}$ & Comments \\
\hline Bache 2001 & 98 & $\begin{array}{l}\text { F: } 84 \% \\
\text { Age: median } \\
69 \\
\text { range } 50 \text { to } 92\end{array}$ & $\begin{array}{l}\text { DRF } \\
\text { Ex: bilateral }\end{array}$ & $\begin{array}{l}\text { Plaster cast } \\
\text { Post-immobili- } \\
\text { sation }\end{array}$ & $\begin{array}{l}\text { Ex: early signs } \\
\text { CRPS-1 or } \\
\text { CTS }\end{array}$ & $\begin{array}{l}\text { Ex: unable to } \\
\text { follow exercise } \\
\text { programme in- } \\
\text { dependently, } \\
\text { impaired men- } \\
\text { tal function, } \\
\text { prior wrist or } \\
\text { joint problems, } \\
\text { physiotherapy } \\
\text { required for } \\
\text { other reason }\end{array}$ & $\begin{array}{l}\text { Population was } \\
\text { without serious } \\
\text { complica- } \\
\text { tions or comor- } \\
\text { bidities }\end{array}$ \\
\hline Basso 1998 & 38 & $\begin{array}{l}\text { F: } 84 \% \\
\text { Age: median } \\
57 \text { and } 63 \\
\text { range } 15 \text { to } 69\end{array}$ & $\begin{array}{l}\text { Colles' } \\
\text { Ex: intra-artic- } \\
\text { ular, se- } \\
\text { vere comminu- } \\
\text { tion, open }\end{array}$ & $\begin{array}{l}\text { Plaster cast } \\
\text { Post-immobili- } \\
\text { sation }\end{array}$ & $\begin{array}{l}\text { Ex: very poor } \\
\text { hand function, } \\
\text { se- } \\
\text { vere injuries in- } \\
\text { cluding to liga- } \\
\text { ments }\end{array}$ & $\begin{array}{l}\text { Ex: unable to } \\
\text { cope with as- } \\
\text { sessments }\end{array}$ & $\begin{array}{l}\text { Population was } \\
\text { without } \\
\text { serious compli- } \\
\text { cations and re- } \\
\text { stricted by frac- } \\
\text { ture type }\end{array}$ \\
\hline Bighea 2013 & 20 & $\begin{array}{l}\text { F: unknown\% } \\
\text { Age: unknown }\end{array}$ & $\begin{array}{l}\text { Wrist } \\
\text { (osteoporotic) }\end{array}$ & $\begin{array}{l}\text { Plaster cast } \\
\text { (probably) } \\
\text { Post-immobili- } \\
\text { sation }\end{array}$ & N/A & N/A & $\begin{array}{l}\text { Abstract report } \\
\text { only }\end{array}$ \\
\hline Brehmer 2014 & 81 & $\begin{array}{l}\text { F: } 73 \% \\
\text { Age: mean } 53 \\
\text { range } 21 \text { to } 83\end{array}$ & $\begin{array}{l}\text { DRF } \\
\text { Ex: } \\
\text { bilateral, prior } \\
\text { fracture, frac- } \\
\text { ture not stable } \\
\text { enough }\end{array}$ & $\begin{array}{l}\text { Surgery - inter- } \\
\text { nal fixation } \\
\text { Post-immobili- } \\
\text { sation (strictly: } \\
\text { post-definitive } \\
\text { treatment) }\end{array}$ & Not stated & Not stated & $\begin{array}{l}\text { Population } \\
\text { limited by suit- } \\
\text { ability for } \\
\text { surgery. Profes- } \\
\text { sional athletes } \\
\text { excluded }\end{array}$ \\
\hline Challis 2007 & 21 & $\begin{array}{l}\text { F: } 76 \% \\
\text { Age: mean } 51\end{array}$ & $\begin{array}{l}\text { DRF } \\
\text { Ex: open, sur- } \\
\text { gical fixation } \\
\text { required }\end{array}$ & $\begin{array}{l}\text { Plaster cast } \\
\text { Defini- } \\
\text { tive treatment } \\
\text { period }\end{array}$ & $\begin{array}{l}\text { Ex: nerve or } \\
\text { tendon damage }\end{array}$ & $\begin{array}{l}\text { Ex: pathology } \\
\text { in intact arm }\end{array}$ & $\begin{array}{l}\text { Popula- } \\
\text { tion limited by } \\
\text { severity of in- } \\
\text { jury }\end{array}$ \\
\hline Cheing 2005 & 83 & $\begin{array}{l}\text { F: } 66 \% \\
\text { Age: mean } 63 \\
\text { range } 17 \text { to } 80\end{array}$ & $\begin{array}{l}\text { DRF } \\
\text { Ex: un- } \\
\text { stable, previous } \\
\text { fracture }\end{array}$ & $\begin{array}{l}\text { Plaster cast } \\
\text { Post-immobili- } \\
\text { sation }\end{array}$ & Ex: CRPS-1 & $\begin{array}{l}\text { Ex: inflamma- } \\
\text { tory arthritis, } \\
\text { prior neurovas- } \\
\text { cular injuries of } \\
\text { hand, various } \\
\text { circulatory dis- }\end{array}$ & $\begin{array}{l}\text { Population was } \\
\text { without serious } \\
\text { complications } \\
\text { or comorbidi- } \\
\text { ties, includ- } \\
\text { ing contraindi- }\end{array}$ \\
\hline
\end{tabular}

Rehabilitation for distal radial fractures in adults (Review) 
Table 2. Summary of study populations (Continued)

\begin{tabular}{|c|c|c|c|c|c|c|c|}
\hline & & & & & & eases etc & $\begin{array}{l}\text { cations for in- } \\
\text { tervention } \\
\text { (PEMF) }\end{array}$ \\
\hline $\begin{array}{l}\text { Christensen } \\
2001\end{array}$ & 32 & $\begin{array}{l}\text { F: } 90 \% \\
\text { Age: mean } 66 \\
\text { range } 42 \text { to } 82\end{array}$ & Colles' & $\begin{array}{l}\text { Plaster cast } \\
\text { Post-immobili- } \\
\text { sation }\end{array}$ & N/A & N/A & $\begin{array}{l}\text { Popula- } \\
\text { tion limitations } \\
\text { not known (no } \\
\text { report of com- } \\
\text { plications) }\end{array}$ \\
\hline Cooper 2001 & 17 & $\begin{array}{l}\text { F: } 94 \% \\
\text { Age: mean } 66 \\
\text { range } 41 \text { to } 81\end{array}$ & $\begin{array}{l}\text { DRF } \\
\text { Ex: open }\end{array}$ & $\begin{array}{l}\text { Plaster cast } \\
\text { Defini- } \\
\text { tive treatment } \\
\text { period }\end{array}$ & $\begin{array}{l}\text { Ex: ex- } \\
\text { tensive soft tis- } \\
\text { sue injuries }\end{array}$ & $\begin{array}{l}\text { Ex: frail elderly } \\
\text { with limited } \\
\text { mobility, im- } \\
\text { paired mental } \\
\text { function, neu- } \\
\text { rological con- } \\
\text { ditions }\end{array}$ & $\begin{array}{l}\text { Population was } \\
\text { without serious } \\
\text { comorbidities }\end{array}$ \\
\hline $\begin{array}{l}\text { Gronlund } \\
1990\end{array}$ & 40 & $\begin{array}{l}\text { F: } 88 \% \\
\text { Age: median } \\
74.5 \\
\text { range } 47 \text { to } 93\end{array}$ & $\begin{array}{l}\text { Colles' } \\
\text { Ex: bilateral, } \\
\text { unstable }\end{array}$ & $\begin{array}{l}\text { Plaster cast } \\
\text { Defini- } \\
\text { tive treatment } \\
\text { period }\end{array}$ & $\begin{array}{l}\text { Ex: neuromus- } \\
\text { cular pain in } \\
\operatorname{limb}\end{array}$ & $\begin{array}{l}\text { Ex: wrist } \\
\text { arthritis, de- } \\
\text { mentia, other } \\
\text { condition pre- } \\
\text { venting partici- } \\
\text { pation }\end{array}$ & $\begin{array}{l}\text { Population was } \\
\text { without serious } \\
\text { complica- } \\
\text { tions or comor- } \\
\text { bidities }\end{array}$ \\
\hline Jongs 2012 & 40 & $\begin{array}{l}\text { F: } 70 \% \\
\text { Age: median } \\
66 \text { and } 58\end{array}$ & $\begin{array}{l}\text { DRF } \\
\text { Ex: bilat- } \\
\text { eral, stable and } \\
\text { healed/healing }\end{array}$ & $\begin{array}{l}\text { Conser- } \\
\text { vative or surgi- } \\
\text { cal treatment } \\
\text { Post-immobili- } \\
\text { sation } \\
\text { ( } 10 \text { weeks from } \\
\text { injury) }\end{array}$ & $\begin{array}{l}\text { Wrist contrac- } \\
\text { ture }\end{array}$ & $\begin{array}{l}\text { Ex: unlikely to } \\
\text { co-operate }\end{array}$ & $\begin{array}{l}\text { Surgeon refer- } \\
\text { ral } \\
\text { Population } \\
\text { limited to those } \\
\text { with wrist con- } \\
\text { tracture } \\
\text { (thus a compli- } \\
\text { cation) and ex- } \\
\text { pected compli- } \\
\text { ance }\end{array}$ \\
\hline Kay 2000 & 40 & $\begin{array}{l}\text { F: } 68 \% \\
\text { Age: mean } 53\end{array}$ & $\begin{array}{l}\text { DRF } \\
\text { Ex: prior frac- } \\
\text { ture }\end{array}$ & $\begin{array}{l}\text { Plaster cast or } \\
\text { pins and plaster } \\
\text { cast } \\
\text { Post-immobili- } \\
\text { sation }\end{array}$ & $\begin{array}{l}\text { Ex: only resid- } \\
\text { ual impairment } \\
\text { from a prior } \\
\text { fracture }\end{array}$ & $\begin{array}{l}\text { Ex: inability to } \\
\text { understand } \\
\text { English }\end{array}$ & $\begin{array}{l}\text { Study popula- } \\
\text { tion } \\
\text { includes those } \\
\text { with complica- } \\
\text { tions (as } \\
\text { reported) }\end{array}$ \\
\hline Kay 2008 & 56 & $\begin{array}{l}\text { F: } 70 \% \\
\text { Age: mean } 55\end{array}$ & $\begin{array}{l}\text { DRF } \\
\text { Ex: bilateral }\end{array}$ & $\begin{array}{l}\text { Plaster cast or } \\
\text { pins and plaster } \\
\text { cast } \\
\text { Post-immobili- } \\
\text { sation }\end{array}$ & $\begin{array}{l}\text { Ex: only resid- } \\
\text { ual impairment } \\
\text { from a prior } \\
\text { fracture }\end{array}$ & $\begin{array}{l}\text { Ex: pre-exist- } \\
\text { ing inflamma- } \\
\text { tory condition, } \\
\text { inability to un- } \\
\text { derstand En- } \\
\text { glish, unable to }\end{array}$ & $\begin{array}{l}\text { Study popula- } \\
\text { tion } \\
\text { includes those } \\
\text { with complica- } \\
\text { tions (as } \\
\text { reported) }\end{array}$ \\
\hline
\end{tabular}

Rehabilitation for distal radial fractures in adults (Review)

Copyright $\Subset 2015$ The Cochrane Collaboration. Published by John Wiley \& Sons, Ltd. 
Table 2. Summary of study populations (Continued)

participate

\begin{tabular}{|c|c|c|c|c|c|c|c|}
\hline $\begin{array}{l}\text { Knygsand- } \\
\text { Roenhoej } \\
2011\end{array}$ & 30 & $\begin{array}{l}\text { F: } 72 \% \\
\text { Age: mean } 78\end{array}$ & $\begin{array}{l}\text { DRF } \\
\text { Ex: bilateral }\end{array}$ & $\begin{array}{l}\text { Plaster cast or } \\
\text { internal or ex- } \\
\text { ternal fixation } \\
\text { Post-immobili- } \\
\text { sation }\end{array}$ & $\begin{array}{l}\text { Subacute } \\
\text { oedema }\end{array}$ & $\begin{array}{l}\text { Ex: mental im- } \\
\text { pairment, } \\
\text { infection, lym- } \\
\text { phedema, dis- } \\
\text { ease of internal } \\
\text { organs }\end{array}$ & $\begin{array}{l}\text { Popula- } \\
\text { tion limited to } \\
\text { those with sub- } \\
\text { acute oedema } \\
\text { (thus a com- } \\
\text { plication) but } \\
\text { without serious } \\
\text { comorbidities }\end{array}$ \\
\hline Krischak 2009 & 48 & $\begin{array}{l}\text { F: } 65 \% \\
\text { Age: mean } 55 \\
\text { range } 18 \text { to } 76\end{array}$ & $\begin{array}{l}\text { DRF } \\
\text { Ex: previous } \\
\text { fracture }\end{array}$ & $\begin{array}{l}\text { Internal fixa- } \\
\text { tion with lock- } \\
\text { ing plate } \\
\text { Post-immobili- } \\
\text { sation (strictly: } \\
\text { post-definitive } \\
\text { treatment) }\end{array}$ & $\begin{array}{l}\text { Ex: CTS, } \\
\text { CRPS-1 }\end{array}$ & $\begin{array}{l}\text { Ex: mental im- } \\
\text { pairment, } \\
\text { function- } \\
\text { ally dependent, } \\
\text { inflammatory } \\
\text { joint disease }\end{array}$ & $\begin{array}{l}\text { Population was } \\
\text { without serious } \\
\text { complications } \\
\text { or comorbidi- } \\
\text { ties and lim- } \\
\text { ited by suitabil- } \\
\text { ity for surgery }\end{array}$ \\
\hline Kuo 2013 & 22 & $\begin{array}{l}\text { F: } 65 \% \\
\text { Age: mean } 62\end{array}$ & $\begin{array}{l}\text { DRF } \\
\text { Ex: open }\end{array}$ & $\begin{array}{l}\text { External } \\
\text { fixation } \\
\text { Defini- } \\
\text { tive treatment } \\
\text { period }\end{array}$ & $\begin{array}{l}\text { Ex: neurologi- } \\
\text { cal defects or } \\
\text { severe soft tis- } \\
\text { sue damage }\end{array}$ & $\begin{array}{l}\text { Ex: rheuma- } \\
\text { tism, arthritis, } \\
\text { neurologi- } \\
\text { cal deficits, pre- } \\
\text { vious hand or } \\
\text { forearm injury }\end{array}$ & $\begin{array}{l}\text { Population was } \\
\text { without serious } \\
\text { complications } \\
\text { or comorbidi- } \\
\text { ties and lim- } \\
\text { ited by suitabil- } \\
\text { ity for surgery }\end{array}$ \\
\hline Lazovic 2012 & 60 & $\begin{array}{l}\text { F: } 100 \% \\
\text { Age: mean } 66 \\
\text { range } 55 \text { to } 78\end{array}$ & $\begin{array}{l}\text { DRF } \\
\text { Ex: } \\
\text { bilateral, intra- } \\
\text { articular, previ- } \\
\text { ous fracture }\end{array}$ & $\begin{array}{l}\text { Plaster cast } \\
\text { Defini- } \\
\text { tive treatment } \\
\text { period }\end{array}$ & None stated & $\begin{array}{l}\text { Ex: in- } \\
\text { flammatory os- } \\
\text { teoarthritis, pe- } \\
\text { ripheral vascu- } \\
\text { lar disease, con- } \\
\text { tra-indications } \\
\text { to PEMF }\end{array}$ & $\begin{array}{l}\text { Population was } \\
\text { without serious } \\
\text { comorbidities, } \\
\text { including con- } \\
\text { traindications } \\
\text { for interven- } \\
\text { tion (PEMF) }\end{array}$ \\
\hline Maciel 2005 & 45 & $\begin{array}{l}\text { F: } 76 \% \\
\text { Age: mean } 56\end{array}$ & DRF & $\begin{array}{l}\text { Plaster cast or } \\
\text { pins and plaster } \\
\text { cast } \\
\text { Post-immobili- } \\
\text { sation }\end{array}$ & $\begin{array}{l}\text { Ex: signs of } \\
\text { CRPS-1 }\end{array}$ & $\begin{array}{l}\text { Ex: inability to } \\
\text { under- } \\
\text { stand English, } \\
\text { psychiatric dis- } \\
\text { or- } \\
\text { der, inflamma- } \\
\text { tory joint con- } \\
\text { dition }\end{array}$ & $\begin{array}{l}\text { Population was } \\
\text { without } \\
\text { serious compli- } \\
\text { cations or some } \\
\text { comorbidities }\end{array}$ \\
\hline Magnus 2013 & 51 & $\begin{array}{l}\text { F: } 100 \% \\
\text { Age: mean } 63\end{array}$ & $\begin{array}{l}\text { DRF } \\
\text { Ex: bilateral }\end{array}$ & $\begin{array}{l}\text { Plaster cast } \\
\text { or surgery and } \\
\text { plaster cast } \\
\text { Defini- } \\
\text { tive treatment }\end{array}$ & None stated & $\begin{array}{l}\text { Ex: } \\
\text { history of up- } \\
\text { per limb neu- } \\
\text { rological prob- } \\
\text { lems, cognitive }\end{array}$ & $\begin{array}{l}\text { Population was } \\
\text { without serious } \\
\text { comorbidities }\end{array}$ \\
\hline
\end{tabular}

Rehabilitation for distal radial fractures in adults (Review) 
Table 2. Summary of study populations (Continued)

\begin{tabular}{|c|c|c|c|c|c|c|c|}
\hline & & & & period & & impairment & \\
\hline Pasila 1974 & 135 & $\begin{array}{l}\text { F: } 93 \% \\
\text { Age: range } 16 \\
\text { to } 65 \text { (criteria) }\end{array}$ & Colles' & $\begin{array}{l}\text { Plaster cast } \\
\text { Defini- } \\
\text { tive treatment } \\
\text { period }\end{array}$ & N/A & N/A & $\begin{array}{l}\text { Study popula- } \\
\text { tion not de- } \\
\text { scribed in } \\
\text { terms of exclu- } \\
\text { sions }\end{array}$ \\
\hline $\begin{array}{l}\text { Rozencwaig } \\
1996\end{array}$ & 7 & $\begin{array}{l}\text { F: unknown } \% \\
\text { Age: unknown }\end{array}$ & DRF & $\begin{array}{l}\text { External } \\
\text { fixation } \\
\text { Post-immobili- } \\
\text { sation }\end{array}$ & N/A & N/A & $\begin{array}{l}\text { Abstract report } \\
\text { only }\end{array}$ \\
\hline Souer 2011 & 94 & $\begin{array}{l}\text { F: } 65 \% \\
\text { Age: mean } 50\end{array}$ & $\begin{array}{l}\text { DRF } \\
\text { Ex: } \\
\text { stable, complex } \\
\text { fracture }\end{array}$ & $\begin{array}{l}\text { Internal fixa- } \\
\text { tion with lock- } \\
\text { ing plate } \\
\text { Post-immobili- } \\
\text { sation (strictly: } \\
\text { post-definitive } \\
\text { treatment) }\end{array}$ & None stated & $\begin{array}{l}\text { Ex: depen- } \\
\text { dency in basic } \\
\text { functioning }\end{array}$ & $\begin{array}{l}\text { Population } \\
\text { limited by in- } \\
\text { dependency in } \\
\text { function } \\
\text { and suitability } \\
\text { for internal fix- } \\
\text { ation }\end{array}$ \\
\hline $\begin{array}{l}\text { Svensson } \\
1993\end{array}$ & 43 & $\begin{array}{l}\text { F: } 100 \% \\
\text { Age: median } \\
72 \\
\text { range } 55 \text { to } 90\end{array}$ & $\begin{array}{l}\text { Colles' } \\
\text { Ex: previous } \\
\text { fracture }\end{array}$ & $\begin{array}{l}\text { Plaster cast } \\
\text { Post-immobili- } \\
\text { sation }\end{array}$ & Ex: CRPS-1 & $\begin{array}{l}\text { Ex: neurologi- } \\
\text { cal disease, dis- } \\
\text { figuring } \\
\text { rheumatic dis- } \\
\text { ease }\end{array}$ & $\begin{array}{l}\text { Referral for re- } \\
\text { habilitation } \\
\text { Population was } \\
\text { without serious } \\
\text { complica- } \\
\text { tions or comor- } \\
\text { bidities }\end{array}$ \\
\hline Taylor 1994 & 30 & $\begin{array}{l}\text { F: } 80 \% \\
\text { Age: mean } 63 \\
\text { range } 39 \text { to } 78\end{array}$ & Colles' & $\begin{array}{l}\text { Plaster cast } \\
\text { Post-immobili- } \\
\text { sation }\end{array}$ & None stated & $\begin{array}{l}\text { Ex: other upper } \\
\text { limb fracture }\end{array}$ & $\begin{array}{l}\text { Study popula- } \\
\text { tion probably } \\
\text { includes those } \\
\text { with complica- } \\
\text { tions }\end{array}$ \\
\hline Toomey 1986 & 24 & $\begin{array}{l}\text { F: } 83 \% \\
\text { Age: mean } 60 \\
\text { range } 40 \text { to } 80\end{array}$ & Colles' & $\begin{array}{l}\text { Plaster cast } \\
\text { Post-immobili- } \\
\text { sation }\end{array}$ & $\begin{array}{l}\text { Ex: CRPS-1 } \\
\text { (shoul- } \\
\text { der-hand syn- } \\
\text { drome) } \\
\text { and other con- } \\
\text { ditions, occu- } \\
\text { pational } \\
\text { therapy for in- } \\
\text { volved hand }\end{array}$ & $\begin{array}{l}\text { Ex: } \\
\text { associated frac- } \\
\text { tures, rheuma- } \\
\text { toid arthritis }\end{array}$ & $\begin{array}{l}\text { Referral for re- } \\
\text { habilitation } \\
\text { Population was } \\
\text { without serious } \\
\text { complications }\end{array}$ \\
\hline $\begin{array}{l}\text { Wakefield } \\
2000\end{array}$ & 96 & $\begin{array}{l}\text { F: } 91 \% \\
\text { Age: mean } 73 \\
\text { range } 55 \text { to } 90\end{array}$ & $\begin{array}{l}\text { DRF } \\
\text { Ex: } \\
\text { bilateral, previ- } \\
\text { ous fracture }\end{array}$ & $\begin{array}{l}\text { Plaster cast } \\
\text { Post-immobili- } \\
\text { sation }\end{array}$ & Ex: CRPS-1 & $\begin{array}{l}\text { Ex: } \\
\text { impaired men- } \\
\text { tal function }\end{array}$ & $\begin{array}{l}\text { Population was } \\
\text { without serious } \\
\text { complica- } \\
\text { tions and cog- }\end{array}$ \\
\hline
\end{tabular}

Rehabilitation for distal radial fractures in adults (Review) 
Table 2. Summary of study populations (Continued)

\begin{tabular}{|c|c|c|c|c|c|c|c|}
\hline & & & & & & & $\begin{array}{l}\text { nitive impair- } \\
\text { ment }\end{array}$ \\
\hline Watt 2000 & 18 & $\begin{array}{l}\text { F: } 94 \% \\
\text { Age: mean } 76\end{array}$ & Colles' & $\begin{array}{l}\text { Plaster cast } \\
\text { Post-immobili- } \\
\text { sation }\end{array}$ & None stated & $\begin{array}{l}\text { Ex: no signif- } \\
\text { icant past his- } \\
\text { tory }\end{array}$ & $\begin{array}{l}\text { Attending out- } \\
\text { patients } \\
\text { Study popula- } \\
\text { tion may have } \\
\text { included com- } \\
\text { plications. It is } \\
\text { not clear what } \\
\text { "past history" } \\
\text { covers }\end{array}$ \\
\hline
\end{tabular}

CRPS-1: complex regional pain syndrome type 1 (often referred to as reflex sympathetic dystrophy in older included studies)

CTS: carpal tunnel syndrome

DRF: distal radius fracture

Ex: excluded

F: \% female

N/A: not available

PEMF: pulsed electromagnetic field

Table 3. Summary of rehabilitation interventions

\begin{tabular}{|c|c|c|c|c|c|c|}
\hline Study ID & What & Who & When & Where & How long & Comments \\
\hline & Intervention & Provider & Started & Location & Duration & $\begin{array}{l}\text { Treatment } \\
\text { for all or con- } \\
\text { trol group par- } \\
\text { ticipants }\end{array}$ \\
\hline Bache 2001 & Physiotherapy & Physiotherapist & $\begin{array}{l}\text { Within a week } \\
\text { of plaster cast re- } \\
\text { moval; } 5 \text {-6 weeks } \\
\text { immobilisation }\end{array}$ & $\begin{array}{l}\text { Outpatients } \\
\text { clinic }\end{array}$ & $\begin{array}{l}\text { Contents and } \\
\text { discharge at dis- } \\
\text { cretion of phys- } \\
\text { iotherapists }\end{array}$ & $\begin{array}{l}\text { All participants } \\
\text { taught home ex- } \\
\text { ercises by physio- } \\
\text { therapist at out- } \\
\text { patients clinic }\end{array}$ \\
\hline Basso 1998 & Ultrasound & Not reported & $\begin{array}{l}\text { After plaster cast } \\
\text { removal; average } \\
4 \text { weeks immo- } \\
\text { bilisation }\end{array}$ & Fracture clinic? & 5 minutes & $\begin{array}{l}\text { Sham control } \\
\text { All partic- } \\
\text { ipants given in- } \\
\text { structions. Phys- } \\
\text { iotherapy only if } \\
\text { poor hand func- } \\
\text { tion }\end{array}$ \\
\hline Bighea 2013 & Physiotherapy & Physiotherapist & $\begin{array}{l}\text { Probably after } 4 \\
\text { weeks immobili- } \\
\text { sation }\end{array}$ & Not stated & $\begin{array}{l}\text { Four weeks; not } \\
\text { sure if contin- } \\
\text { ued after exami- } \\
\text { nation }\end{array}$ & $\begin{array}{l}\text { Instructions for } \\
\text { a home exercise } \\
\text { programme }\end{array}$ \\
\hline
\end{tabular}


Table 3. Summary of rehabilitation interventions

\begin{tabular}{|c|c|c|c|c|c|c|}
\hline Brehmer 2014 & $\begin{array}{l}\text { 'Accelerated' } \\
\text { post-op rehabili- } \\
\text { tation started at } \\
2 \text { weeks }\end{array}$ & Hand therapist & $\begin{array}{l}\text { At } 2 \text { weeks post- } \\
\text { surgery }\end{array}$ & $\begin{array}{l}\text { Ambulatory } \\
\text { surgery centre }\end{array}$ & Over 5 weeks. & $\begin{array}{l}\text { Both groups be- } \\
\text { gan with gen- } \\
\text { tle active range } \\
\text { of motion at } 3 \\
\text { to } 5 \text { days after } \\
\text { surgery; remov- } \\
\text { able cus- } \\
\text { tom splint ap- } \\
\text { plied. Same re- } \\
\text { habilitation pro- } \\
\text { gramme applied } \\
\text { in control group } \\
\text { but started at } 6 \\
\text { weeks and con- } \\
\text { densed to } 3 \\
\text { weeks }\end{array}$ \\
\hline Challis 2007 & $\begin{array}{l}\text { Cyclic pneu- } \\
\text { matic soft tis- } \\
\text { sue compression } \\
\text { during cast im- } \\
\text { mobilisation (in- } \\
\text { flatable cuff } \\
\text { placed under the } \\
\text { plaster cast) }\end{array}$ & $\begin{array}{l}\text { Probably physio- } \\
\text { therapist for in- } \\
\text { structions }\end{array}$ & $\begin{array}{l}\text { After week in a } \\
\text { split cast (mean } \\
9 . \\
4 \text { days after frac- } \\
\text { ture), upon re- } \\
\text { placement with a } \\
\text { full forearm plas- } \\
\text { ter }\end{array}$ & $\begin{array}{l}\text { At home } \\
\text { after instructions } \\
\text { at fracture clinic }\end{array}$ & $\begin{array}{l}\text { Pump ap- } \\
\text { plied for } 10 \text { min- } \\
\text { utes each morn- } \\
\text { ing and evening } \\
\text { during } 5 \text { weeks } \\
\text { full forearm cast } \\
\text { immobilisation }\end{array}$ & $\begin{array}{l}\text { Device also in- } \\
\text { corporated into } \\
\text { control group } \\
\text { cast. Both groups } \\
\text { received instruc- } \\
\text { tions for hand } \\
\text { exercises during } \\
\text { 6-week immo- } \\
\text { bilisation period } \\
\text { and were given } \\
\text { a 4-week exercise } \\
\text { programme after } \\
\text { cast removal }\end{array}$ \\
\hline Cheing 2005 & $\begin{array}{l}\text { Pulsed elec- } \\
\text { tromagnetic field } \\
\text { (PEMF) or ice, } \\
\text { or both }\end{array}$ & Physiotherapist & $\begin{array}{l}\text { After plaster cast } \\
\text { removal; average } \\
6 \text { weeks immo- } \\
\text { bilisation }\end{array}$ & $\begin{array}{l}\text { Outpatients } \\
\text { clinic? }\end{array}$ & $\begin{array}{l}30 \text { minutes of } \\
\text { each for } 5 \text { con- } \\
\text { secutive days }\end{array}$ & $\begin{array}{l}\text { Sham PEMF. All } \\
\text { participants } \\
\text { taught home ex- } \\
\text { ercises by physio- } \\
\text { therapist at out- } \\
\text { patients clinic }\end{array}$ \\
\hline $\begin{array}{l}\text { Christensen } \\
2001\end{array}$ & $\begin{array}{l}\text { Occupational } \\
\text { therapy }\end{array}$ & $\begin{array}{l}\text { Occupational } \\
\text { therapist }\end{array}$ & $\begin{array}{l}\text { After plaster cast } \\
\text { removal; } 5 \text { weeks } \\
\text { immobilisation }\end{array}$ & $\begin{array}{l}\text { Rheumatology } \\
\text { department }\end{array}$ & $\begin{array}{l}\text { Twice weekly } \\
\text { sessions until } \\
\text { progress plateau }\end{array}$ & $\begin{array}{l}\text { All had instruc- } \\
\text { tions for home } \\
\text { exercises by oc- } \\
\text { cupational thera- } \\
\text { pist }\end{array}$ \\
\hline Cooper 2001 & $\begin{array}{l}\text { 'Early' therapy - } \\
\text { oedema manage- } \\
\text { ment, exercises, } \\
\text { monitoring, in- } \\
\text { formation }\end{array}$ & Hand therapist & $\begin{array}{l}\text { After } \\
\text { initial treatment; } \\
\text { within } 4 \text { days of } \\
\text { fracture }\end{array}$ & Clinic? & $\begin{array}{l}\text { Dur- } \\
\text { ing immobilisa- } \\
\text { tion, routinely } 4 \\
\text { weeks but up to } \\
6 \text { weeks as re- }\end{array}$ & $\begin{array}{l}\text { All had instruc- } \\
\text { tions for home } \\
\text { programme: skin } \\
\text { care, exercises, } \\
\text { oedema control }\end{array}$ \\
\hline
\end{tabular}

Rehabilitation for distal radial fractures in adults (Review)

Copyright $\odot 2015$ The Cochrane Collaboration. Published by John Wiley \& Sons, Ltd. 
quired. Weekly at fracture clinic. contact Post-immobilisation care comprised an individualised home programme and, if required, attendance of hand therapy group

\begin{tabular}{|c|c|c|c|c|c|c|}
\hline Gronlund 1990 & $\begin{array}{l}\text { Occupational } \\
\text { therapy }\end{array}$ & $\begin{array}{l}\text { Occupational } \\
\text { therapist }\end{array}$ & $\begin{array}{l}\text { After initial } \\
\text { treatment; } 1 \text { to } 3 \\
\text { days after plaster } \\
\text { cast application }\end{array}$ & $\begin{array}{l}\text { Rheumatoid dis- } \\
\text { orders outpa- } \\
\text { tients clinic }\end{array}$ & $\begin{array}{l}\text { Single ses- } \\
\text { sion with referral } \\
\text { as required }\end{array}$ & $\begin{array}{l}\text { All had instruc- } \\
\text { tions for home } \\
\text { exercises by oc- } \\
\text { cupational thera- } \\
\text { pist } \\
\text { at casualty ward. } \\
\text { Referral for oc- } \\
\text { cupational ther- } \\
\text { apy if required } \\
\text { after plaster cast } \\
\text { removal }\end{array}$ \\
\hline Jongs 2012 & $\begin{array}{l}\text { A dynamic splint } \\
\text { fitted, } \\
\text { which stretched } \\
\text { the wrist into ex- } \\
\text { tension but al- } \\
\text { lowed intermit- } \\
\text { tent movement }\end{array}$ & Physiotherapist & $\begin{array}{l}\text { After } 10 \\
\text { weeks from frac- } \\
\text { ture; post- } \\
\text { immobilisation }\end{array}$ & $\begin{array}{l}\text { Physiotherapy } \\
\text { and then home }\end{array}$ & $\begin{array}{l}8 \text { weeks. Partic- } \\
\text { ipants were in- } \\
\text { structed to wear } \\
\text { the splint for as } \\
\text { long as possi- } \\
\text { ble during the } \\
\text { day, aiming for } \\
\text { at least six hours } \\
\text { overall per day }\end{array}$ & $\begin{array}{l}\text { Both groups re- } \\
\text { ceived } \\
\text { routine care con- } \\
\text { sisting of exer- } \\
\text { cises and advice } \\
\text { for } 8 \text { weeks, with } \\
\text { fortnightly mon- } \\
\text { itoring. Partici- } \\
\text { pants continued } \\
\text { with the exercises } \\
\text { and advice unsu- } \\
\text { pervised until } 12 \\
\text { weeks }\end{array}$ \\
\hline Kay 2000 & $\begin{array}{l}\text { Passive mobilisa- } \\
\text { tion }\end{array}$ & Physiotherapist & $\begin{array}{l}\text { After plaster cast } \\
\text { or plaster \& pins } \\
\text { removal; about } 6 \\
\text { weeks immobili- } \\
\text { sation }\end{array}$ & Physiotherapy & $\begin{array}{l}\text { 6-week course: } 2 \\
\mathrm{x} \text { week for } 3 \\
\text { weeks, } 1 \text { x week } \\
\text { for } 3 \text { weeks }\end{array}$ & $\begin{array}{l}\text { All } \\
\text { attended physio- } \\
\text { therapy for ini- } \\
\text { tial advice and } \\
\text { instructions for } \\
\text { home exercises } \\
\text { given by physio- } \\
\text { therapists }\end{array}$ \\
\hline Kay 2008 & $\begin{array}{l}\text { One ses- } \\
\text { sion of physio- } \\
\text { therapy; primar- } \\
\text { ily advice and in- } \\
\text { structions }\end{array}$ & Physiotherapist & $\begin{array}{l}\text { After plaster cast } \\
\text { or plaster \& pins } \\
\text { removal; about } 6 \\
\text { weeks immobili- }\end{array}$ & Physiotherapy & Single session & $\begin{array}{l}\text { Control group } \\
\text { had no physio- } \\
\text { therapy in- } \\
\text { put. Both groups }\end{array}$ \\
\hline
\end{tabular}


Table 3. Summary of rehabilitation interventions

\begin{tabular}{|c|c|c|c|c|c|c|}
\hline & & & sation & & & $\begin{array}{l}\text { had initial as- } \\
\text { sessment and re- } \\
\text { view at } 3 \text { and } 6 \\
\text { weeks by an ex- } \\
\text { perienced hand } \\
\text { therapist }\end{array}$ \\
\hline $\begin{array}{l}\text { Knygsand- } \\
\text { Roenhoej } 2011\end{array}$ & $\begin{array}{l}\text { Modified "man- } \\
\text { ual edema mobi- } \\
\text { lization" (MEM) } \\
\text {. Massage then } \\
\text { exercise in seg- } \\
\text { ment just mas- } \\
\text { saged }\end{array}$ & $\begin{array}{l}\text { Occupational } \\
\text { therapists }\end{array}$ & $\begin{array}{l}\text { Post-cast immo- } \\
\text { bilisation, or } \\
\text { surgery (internal } \\
\text { or external fixa- } \\
\text { tion): between } 4 \\
\text { to } 10 \text { weeks }\end{array}$ & $\begin{array}{l}\text { Depart- } \\
\text { ment of occupa- } \\
\text { tional therapy }\end{array}$ & 6-week course & $\begin{array}{l}\text { 'Tra- } \\
\text { ditional' oedema } \\
\text { treatment } \\
\text { In all, oedema } \\
\text { treatment was } \\
\text { performed three } \\
\text { times a week for } \\
\text { four } \\
\text { weeks and then } \\
\text { twice a week for } \\
\text { two weeks. Sub- } \\
\text { sequently, until } \\
\text { acceptable func- } \\
\text { tion reached. All } \\
\text { patients } \\
\text { had ROM and } \\
\text { strengthening } \\
\text { therapy at hospi- } \\
\text { tal and home ex- } \\
\text { ercises }\end{array}$ \\
\hline Krischak 2009 & $\begin{array}{l}\text { Physiother- } \\
\text { apy (at discre- } \\
\text { tion of therapist; } \\
\text { but instructed to } \\
\text { implement exer- } \\
\text { cises that could } \\
\text { be done } \\
\text { by the patients at } \\
\text { home) }\end{array}$ & $\begin{array}{l}\text { Physio- } \\
\text { therapist (partic- } \\
\text { ipant choice) }\end{array}$ & $\begin{array}{l}\text { One week post- } \\
\text { surgery; } \\
2 \text { weeks of splint } \\
\text { from surgery (re- } \\
\text { moved for ther- } \\
\text { apy) }\end{array}$ & Physiotherapy & $\begin{array}{l}12 \text { sessions last- } \\
\text { ing } 20 \text { to } 30 \text { min- } \\
\text { utes each, over a } \\
6 \text {-week period }\end{array}$ & $\begin{array}{l}\text { Instruc- } \\
\text { tion for home } \\
\text { exercises in per- } \\
\text { son with an il- } \\
\text { lustrated exercise } \\
\text { guidance book- } \\
\text { let and training } \\
\text { diary provided. } \\
\text { Progres- } \\
\text { sive week-based } \\
\text { exercise plan. In- } \\
\text { structions for } \\
\text { per- } \\
\text { forming groups } \\
\text { of exercises, re- } \\
\text { quiring approxi- } \\
\text { mately } 20 \text { min- } \\
\text { utes, in morning } \\
\text { and evening }\end{array}$ \\
\hline
\end{tabular}

Rehabilitation for distal radial fractures in adults (Review)

Copyright $\odot 2015$ The Cochrane Collaboration. Published by John Wiley \& Sons, Ltd. 
Table 3. Summary of rehabilitation interventions

\begin{tabular}{|c|c|c|c|c|c|c|}
\hline Kuo 2013 & $\begin{array}{l}\text { Early } \\
\text { mobilisation of } \\
\text { digits; including } \\
\text { massage, passive } \\
\text { and active range } \\
\text { of motion of fin- } \\
\text { ger joints, activ- } \\
\text { ity of daily living } \\
\text { training }\end{array}$ & $\begin{array}{l}\text { Occupational } \\
\text { therapist }\end{array}$ & $\begin{array}{l}\text { In the week after } \\
\text { surgery }\end{array}$ & $\begin{array}{l}\text { Depart- } \\
\text { ment of occupa- } \\
\text { tional therapy? }\end{array}$ & $\begin{array}{l}3 \\
\text { x } 45 \text {-minute ses- } \\
\text { sions each week } \\
\text { for } 6 \text { weeks until } \\
\text { removal of exter- } \\
\text { nal fixator }\end{array}$ & $\begin{array}{l}\text { Active ROM of } \\
\text { shoulder and el- } \\
\text { bow joints; and } \\
\text { hand joints after } \\
\text { week } 3 \text { onwards } \\
\text { All had standard } \\
\text { progressive reha- } \\
\text { bilitation pro- } \\
\text { gramme, includ- } \\
\text { ing scar man- } \\
\text { agement, active } \\
\text { and passive wrist } \\
\text { range of motion } \\
\text { and strengthen- } \\
\text { ing for six weeks } \\
\text { after fixator re- } \\
\text { moval }\end{array}$ \\
\hline Lazovic 2012 & $\begin{array}{l}\text { Pulsed elec- } \\
\text { tromagnetic field } \\
(\text { PEMF) }\end{array}$ & Not stated & $\begin{array}{l}\text { After clinical and } \\
\text { ra- } \\
\text { diographic check } \\
\text { at } 7 \text { days (mean } \\
8 \text { days post-frac- } \\
\text { ture) }\end{array}$ & $\begin{array}{l}\text { Rehabilitation } \\
\text { clinic }\end{array}$ & $\begin{array}{l}\text { Provided } 5 \text { days a } \\
\text { week for } 2 \text { weeks, } \\
30 \text { minutes daily }\end{array}$ & $\begin{array}{l}\text { No other therapy } \\
\text { control } \\
\text { Instructions } \\
\text { for home exer- } \\
\text { cise programme } \\
\text { (unin- } \\
\text { volved joint mo- } \\
\text { bilisation) dur- } \\
\text { ing cast immo- } \\
\text { bil- } \\
\text { isation (mean } 28 \\
\text { days). Then in- } \\
\text { structed to per- } \\
\text { form light activ- } \\
\text { ities in the pain- } \\
\text { free range of } \\
\text { movement until } \\
\text { the follow-up (2 } \\
\text { to } 3 \text { days). Wrist } \\
\text { elevation for in- } \\
\text { creased oedema }\end{array}$ \\
\hline Maciel 2005 & $\begin{array}{l}\text { "Activity- } \\
\text { focussed" phys- } \\
\text { iotherapy }\end{array}$ & Physiotherapist & $\begin{array}{l}\text { After plaster cast } \\
\text { or plaster \& pins } \\
\text { (K-wire) } \\
\text { removal; about } 6 \\
\text { weeks immobili- } \\
\text { sation }\end{array}$ & Physiotherapy? & $\begin{array}{l}\text { Up to } 6 \text { weeks, } \\
\text { until return to } \\
\text { regular wrist ac- } \\
\text { tivity }\end{array}$ & $\begin{array}{l}\text { All par- } \\
\text { ticipants taught } \\
\text { routine exercises } \\
\text { by physiothera- } \\
\text { pist } \\
\text { at fracture clinic } \\
\text { on day of cast re- } \\
\text { moval. Control }\end{array}$ \\
\hline
\end{tabular}




\begin{tabular}{|c|c|c|c|c|c|c|}
\hline & & & & & & $\begin{array}{l}\text { group had sin- } \\
\text { gle session of ad- } \\
\text { vice on exercises } \\
\text { from physiother- } \\
\text { apist within one } \\
\text { week of trial en- } \\
\text { try }\end{array}$ \\
\hline Magnus 2013 & $\begin{array}{l}\text { Strength } \\
\text { training of oppo- } \\
\text { site hand (cross- } \\
\text { education) }\end{array}$ & $\begin{array}{l}\text { Orthopaedic } \\
\text { surgeon }\end{array}$ & $\begin{array}{l}\text { Immediately af- } \\
\text { ter first clinic } \\
\text { visit }\end{array}$ & $\begin{array}{l}\text { Instructions at } \\
\text { fracture clinic } \\
\text { Unsupervised } \\
\text { home exercises }\end{array}$ & 26 weeks & $\begin{array}{l}\text { All partic- } \\
\text { ipants had stan- } \\
\text { dard rehabilita- } \\
\text { tion, with three } \\
\text { successful paper- } \\
\text { based exer- } \\
\text { cise protocols for } \\
\text { the injured arm: } \\
\text { during } \\
\text { cast use (approx- } \\
\text { imately } 6 \text { weeks) } \\
6 \text {-8 weeks and } 9 \\
\text { weeks onwards. } \\
\text { Patients encour- } \\
\text { aged to continue } \\
\text { exercise to } 26 \\
\text { weeks) }\end{array}$ \\
\hline Pasila 1974 & $\begin{array}{l}\text { Advice \& } \\
\text { instructions pro- } \\
\text { vided by physio- } \\
\text { therapist; super- } \\
\text { vised exercises }\end{array}$ & Physiotherapist & $\begin{array}{l}\text { After } \\
\text { initial treatment; } \\
\text { day after plaster } \\
\text { cast application }\end{array}$ & $\begin{array}{l}\text { Physical } \\
\text { medicine depart- } \\
\text { ment }\end{array}$ & $\begin{array}{l}\text { Until physio- } \\
\text { therapist consid- } \\
\text { ered patient able } \\
\text { to carry on unsu- } \\
\text { pervised }\end{array}$ & $\begin{array}{l}\text { Control } \\
\text { group had same } \\
\text { oral \& written } \\
\text { instructions pro- } \\
\text { vided by physi- } \\
\text { cian/surgeon }\end{array}$ \\
\hline $\begin{array}{l}\text { Rozencwaig } \\
1996\end{array}$ & $\begin{array}{l}\text { Continuous pas- } \\
\text { sive motion }\end{array}$ & $\begin{array}{l}\text { "Therapist", } \\
\text { proba- } \\
\text { bly occupational } \\
\text { therapist }\end{array}$ & $\begin{array}{l}\text { After exter- } \\
\text { nal fixation for } 6 \\
\text { to } 8 \text { weeks }\end{array}$ & $\begin{array}{l}\text { Not reported. } \\
\text { Clinic? }\end{array}$ & $\begin{array}{l}\text { Not reported. } \\
\text { Until criteria for } \\
\text { recovery met? }\end{array}$ & $\begin{array}{l}\text { All partic- } \\
\text { ipants had occu- } \\
\text { pational therapy }\end{array}$ \\
\hline Souer 2011 & $\begin{array}{l}\text { Occupational } \\
\text { therapy }\end{array}$ & $\begin{array}{l}\text { "Hand } \\
\text { therapist" }\end{array}$ & $\begin{array}{l}\text { At the first post- } \\
\text { operative visit to } \\
\text { the surgeon's of- } \\
\text { fice after volar } \\
\text { plate fixation }\end{array}$ & $\begin{array}{l}\text { Not reported. } \\
\text { Clinic? }\end{array}$ & $\begin{array}{l}\text { Frequency and } \\
\text { duration of } \\
\text { the re- } \\
\text { habilitation pro- } \\
\text { gramme were at } \\
\text { the discretion of } \\
\text { the treating hand } \\
\text { therapist }\end{array}$ & $\begin{array}{l}\text { Control group } \\
\text { had surgeon-di- } \\
\text { rected home } \\
\text { exercise } \\
\text { programme; also } \\
\text { supplied with } \\
\text { wrist splint }\end{array}$ \\
\hline
\end{tabular}


Table 3. Summary of rehabilitation interventions

\begin{tabular}{|c|c|c|c|c|c|c|}
\hline Svensson 1993 & $\begin{array}{l}\text { Intermittent } \\
\text { pneumatic com- } \\
\text { pression }\end{array}$ & $\begin{array}{l}\text { Occupational } \\
\text { therapist }\end{array}$ & $\begin{array}{l}\text { After plaster cast } \\
\text { re- } \\
\text { moval; treatment } \\
\text { started } 1-46 \text { days } \\
\text { afterwards }\end{array}$ & $\begin{array}{l}\text { Rheumatologi- } \\
\text { cal ward }\end{array}$ & $\begin{array}{l}20 \text { minutes be- } \\
\text { fore each occu- } \\
\text { pational } \\
\text { therapy sessions: } \\
\text { thrice weekly for } \\
3 \text { weeks }\end{array}$ & $\begin{array}{l}\text { All partic- } \\
\text { ipants had occu- } \\
\text { pational therapy. } \\
\text { In- } \\
\text { structions for ex- } \\
\text { ercises given on } \\
\text { first session }\end{array}$ \\
\hline Taylor 1994 & $\begin{array}{l}\text { Passive mobilisa- } \\
\text { tion }\end{array}$ & Physiotherapist & $\begin{array}{l}\text { Within } 3 \text { work- } \\
\text { ing days after } \\
\text { plaster cast re- } \\
\text { moval; } 6 \text { weeks } \\
\text { immobilisation }\end{array}$ & $\begin{array}{l}\text { Physiotherapy } \\
\text { department }\end{array}$ & $\begin{array}{l}5 \text { minutes dur- } \\
\text { ing twice weekly } \\
\text { physiotherapy } \\
\text { sessions; sessions } \\
\text { ended } \\
\text { when acceptable } \\
\text { function or func- } \\
\text { tion plateau }\end{array}$ & $\begin{array}{l}\text { Sham } \\
\text { control: soft tis- } \\
\text { sue massage. All } \\
\text { participants had } \\
\text { physiotherapy }\end{array}$ \\
\hline Toomey 1986 & Whirlpool & Physiotherapist & $\begin{array}{l}\text { Within } 6 \text { days af- } \\
\text { ter plaster cast re- } \\
\text { moval; average } 6 \\
\text { weeks immobili- } \\
\text { sation }\end{array}$ & $\begin{array}{l}\text { Physical } \\
\text { medicine depart- } \\
\text { ment }\end{array}$ & $\begin{array}{l}\text { First } 15 \text { minutes } \\
\text { scheduled twice } \\
\text { weekly phys- } \\
\text { iotherapy over } 6 \\
\text { weeks }\end{array}$ & $\begin{array}{l}\text { Control group: } \\
\text { towel wrap for } \\
15 \text { minutes }\end{array}$ \\
\hline Wakefield 2000 & Physiotherapy & Physiotherapist & $\begin{array}{l}\text { After plaster cast } \\
\text { removal; average } \\
37 \text { days immo- } \\
\text { bilisation }\end{array}$ & $\begin{array}{l}\text { Local hospital or } \\
\text { clinic }\end{array}$ & $\begin{array}{l}\text { Contents and } \\
\text { discharge at dis- } \\
\text { cretion of phys- } \\
\text { iotherapists }\end{array}$ & $\begin{array}{l}\text { All participants } \\
\text { taught home ex- } \\
\text { ercises by physio- } \\
\text { therapist at frac- } \\
\text { ture clinic }\end{array}$ \\
\hline Watt 2000 & Physiotherapy & $\begin{array}{l}\text { Therapist; prob- } \\
\text { ably physiother- } \\
\text { apist }\end{array}$ & $\begin{array}{l}\text { After plaster cast } \\
\text { removal; average } \\
43 \text { days immo- } \\
\text { bilisation }\end{array}$ & $\begin{array}{l}\text { Physiotherapy } \\
\text { department }\end{array}$ & $\begin{array}{l}\text { Contents and } \\
\text { discharge at dis- } \\
\text { cretion of thera- } \\
\text { pists }\end{array}$ & $\begin{array}{l}\text { Con- } \\
\text { trol group had } \\
\text { oral and writ- } \\
\text { ten instructions } \\
\text { provided by sur- } \\
\text { geon/registrar }\end{array}$ \\
\hline
\end{tabular}

PEMF: pulsed electromagnetic field

ROM: range of motion

Table 4. Assessment of items relating to applicability of trial findings

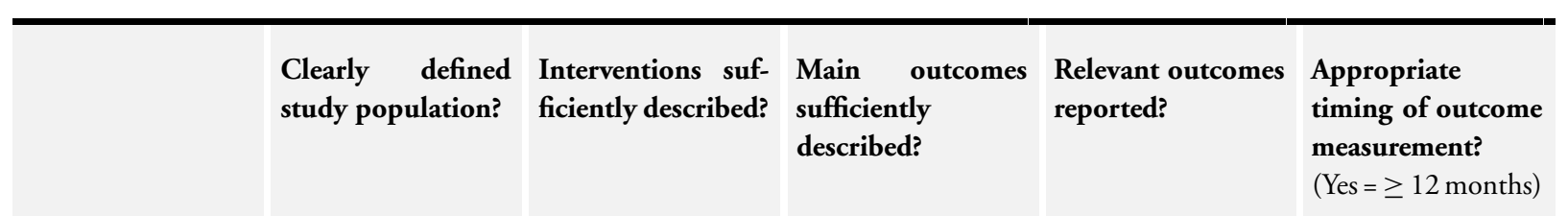

Rehabilitation for distal radial fractures in adults (Review) 
Table 4. Assessment of items relating to applicability of trial findings

\begin{tabular}{|c|c|c|c|c|c|}
\hline Bache 2001 & Yes & $\begin{array}{l}\text { Partial: "semi-stan- } \\
\text { dardised" } \quad \text { with } \\
\text { agreed discharge cri- } \\
\text { teria. (Pragmatic } \\
\text { trial) }\end{array}$ & Yes & Yes & No: 12 weeks \\
\hline Basso 1998 & Yes & $\begin{array}{l}\text { Partial: care } \\
\text { providers not iden- } \\
\text { tified. (Yes for inter- } \\
\text { vention) }\end{array}$ & Yes & $\begin{array}{l}\text { Partial: no PROMs, } \\
\text { limited other out- } \\
\text { comes but evidence } \\
\text { of systematic ap- } \\
\text { proach }\end{array}$ & No: 8 to 16 weeks \\
\hline Bighea 2013 & $\begin{array}{l}\text { No: osteoporotic } \\
\text { 'wrist' fracture }\end{array}$ & $\begin{array}{l}\text { Partial: Not clear } \\
\text { when started (prob- } \\
\text { ably post-immobili- } \\
\text { sation); insufficient } \\
\text { details of providers }\end{array}$ & $\begin{array}{l}\text { Partial: limited in- } \\
\text { formation }\end{array}$ & $\begin{array}{l}\text { Yes: although insuf- } \\
\text { ficient details }\end{array}$ & $\begin{array}{l}\text { No: } 4 \text { weeks post- } \\
\text { cast removal }\end{array}$ \\
\hline Brehmer 2014 & $\begin{array}{l}\text { Partial: no detail on } \\
\text { decision for } \\
\text { non-surgical Table } 9 \\
\text { treatment }\end{array}$ & Yes & Yes & Yes & Partial: 6 months \\
\hline Challis 2007 & $\begin{array}{l}\text { Partial: no detail on } \\
\text { decision for non- } \\
\text { surgical treatment }\end{array}$ & Yes & Yes & Partial: no PROMs & No: 10 weeks \\
\hline Cheing 2005 & Yes & Yes & Yes & $\begin{array}{l}\text { Partial: no PROMs } \\
\text { except pain }\end{array}$ & No: 5 days \\
\hline Christensen 2001 & $\begin{array}{l}\text { Partial: Colles } \\
\text { treated by below-el- } \\
\text { bow cast. No exclu- } \\
\text { sion criteria }\end{array}$ & $\begin{array}{l}\text { Partial: insufficient } \\
\text { details of occupa- } \\
\text { tional therapy }\end{array}$ & $\begin{array}{l}\text { Partial: insufficient } \\
\text { detail }\end{array}$ & $\begin{array}{l}\text { Partial: no PROMs, } \\
\text { reliance on } \\
\text { Gartland and Wer- } \\
\text { ley outcome mea- } \\
\text { sure }\end{array}$ & Partial: 9 months \\
\hline Cooper 2001 & Yes & Yes & Yes & Yes & $\begin{array}{l}\text { No: } 4 \text { weeks post- } \\
\text { cast removal }\end{array}$ \\
\hline Gronlund 1990 & Yes & $\begin{array}{l}\text { Partial: insufficient } \\
\text { details of occupa- } \\
\text { tional therapy }\end{array}$ & Yes & $\begin{array}{l}\text { Partial: no PROMs, } \\
\text { reliance on modified } \\
\text { Gartland and Wer- } \\
\text { ley outcome mea- } \\
\text { sure }\end{array}$ & No: 13 weeks \\
\hline Jongs 2012 & Yes & Yes & Yes & Yes & No: 12 weeks \\
\hline
\end{tabular}


Table 4. Assessment of items relating to applicability of trial findings (Continued)

\begin{tabular}{|c|c|c|c|c|c|}
\hline Kay 2000 & Yes & Yes & Yes & $\begin{array}{l}\text { Partial: subjective } \\
\text { functional scale was } \\
\text { new in the study }\end{array}$ & No: 6 weeks \\
\hline Kay 2008 & Yes & Yes & Yes & Yes & No: 6 weeks \\
\hline $\begin{array}{l}\text { Knygsand- } \\
\text { Roenhoej } 2011\end{array}$ & Yes & Yes & Yes & Yes & Partial: 6 months \\
\hline Krischak 2009 & Yes & $\begin{array}{l}\text { No: choice of phys- } \\
\text { iotherapist by pa- } \\
\text { tient; the contents } \\
\text { of the } 12 \text { sessions } \\
\text { of therapy was at } \\
\text { the discretion of the } \\
\text { physiotherapist, al- } \\
\text { though with an in- } \\
\text { struction to imple- } \\
\text { ment exercises that } \\
\text { could be done by } \\
\text { the patients unas- } \\
\text { sisted at home } \\
\text { Home exercise } \\
\text { group intervention } \\
\text { was well described } \\
\text { and illustrated }\end{array}$ & Yes & Yes & No: 6 weeks \\
\hline Kuo 2013 & $\begin{array}{l}\text { Yes: although no de- } \\
\text { tail on indication for } \\
\text { surgery }\end{array}$ & Yes & Yes & $\begin{array}{l}\text { Yes: the Man- } \\
\text { ual Ability Measure- } \\
36 \text { is an acceptable } \\
\text { measure of function }\end{array}$ & No: 12 weeks \\
\hline Lazovic 2012 & Yes & $\begin{array}{l}\text { Partial: care } \\
\text { providers not iden- } \\
\text { tified }\end{array}$ & Yes & Yes & $\begin{array}{l}\text { No: } 2 \text { to } 3 \text { days after } \\
\text { cast removal }\end{array}$ \\
\hline Maciel 2005 & Yes & Yes & Yes & Yes & Partial: 24 weeks \\
\hline Magnus 2013 & $\begin{array}{l}\text { Partial: no details on } \\
\text { fracture type or de- } \\
\text { tail on indication for } \\
\text { surgery }\end{array}$ & Yes & Yes & Yes & Partial: 26 weeks \\
\hline Pasila 1974 & $\begin{array}{l}\text { Partial: not fully de- } \\
\text { fined - "typical" } \\
\text { Colles fracture and } \\
\text { cast and < } 65 \text { years }\end{array}$ & $\begin{array}{l}\text { Partial: exercise pro- } \\
\text { tocol not described }\end{array}$ & Yes & $\begin{array}{l}\text { Partial: no PROMs, } \\
\text { "subjective" attitude } \\
\text { not described }\end{array}$ & No: 12 weeks \\
\hline
\end{tabular}

Rehabilitation for distal radial fractures in adults (Review) 
Table 4. Assessment of items relating to applicability of trial findings (Continued)

\begin{tabular}{|c|c|c|c|c|c|}
\hline Rozencwaig 1996 & $\begin{array}{l}\text { Partial: no informa- } \\
\text { tion on participa- } \\
\text { tions other than un- } \\
\text { stable fractures and } \\
\text { external fixation }\end{array}$ & $\begin{array}{l}\text { No: insufficient in- } \\
\text { formation }\end{array}$ & $\begin{array}{l}\text { No: very limited in- } \\
\text { formation }\end{array}$ & $\begin{array}{l}\text { No: no PROMs, in- } \\
\text { adequate outcome } \\
\text { assessment }\end{array}$ & $\begin{array}{l}\text { No: just up to ini- } \\
\text { tial recovery (a few } \\
\text { weeks) }\end{array}$ \\
\hline Souer 2011 & Yes & $\begin{array}{l}\text { No: while formal } \\
\text { occupational ther- } \\
\text { apy included super- } \\
\text { vised exercises, the } \\
\text { content, frequency } \\
\text { and duration of oc- } \\
\text { cupational therapy } \\
\text { was at the discretion } \\
\text { of the hand thera- } \\
\text { pist } \\
\text { Home } \\
\text { exercise group inter- } \\
\text { vention was well de- } \\
\text { scribed }\end{array}$ & Yes & Yes & Partial: 6 months \\
\hline Svensson 1993 & Yes & Yes & Yes & $\begin{array}{l}\text { Partial: visual ana- } \\
\text { logue scale for pain } \\
\text { and use of hand in } \\
\text { daily activities but } \\
\text { no PROMs. Other- } \\
\text { wise, clearly a sys- } \\
\text { tematic approach to } \\
\text { data collection }\end{array}$ & No: 3 months \\
\hline Taylor 1994 & Yes & $\begin{array}{l}\text { Yes: note that ther- } \\
\text { apists decided upon } \\
\text { the details of the } \\
\text { specific passive mo- } \\
\text { bilisation treatment }\end{array}$ & Yes & $\begin{array}{l}\text { Partial: no PROMs, } \\
\text { but clearly a sys- } \\
\text { tematic approach to } \\
\text { data collection for } \\
\text { the few outcomes }\end{array}$ & $\begin{array}{l}\text { No: until discharge } \\
\text { (mean } 26 \text { days) }\end{array}$ \\
\hline Toomey 1986 & Yes & Yes & Yes & $\begin{array}{l}\text { Partial: no PROMs, } \\
\text { but clearly a sys- } \\
\text { tematic approach to } \\
\text { data collection }\end{array}$ & No: 6 weeks \\
\hline Wakefield 2000 & Yes & $\begin{array}{l}\text { No: while this was a } \\
\text { pragmatic trial there } \\
\text { were insufficient de- } \\
\text { tails on the interven- } \\
\text { tions and reported } \\
\text { variation in physio- } \\
\text { therapy between the } \\
\text { trial centres }\end{array}$ & Yes & $\begin{array}{l}\text { Partial: no PROMs, } \\
\text { but clearly system- } \\
\text { atic and otherwise } \\
\text { comprehensive }\end{array}$ & Partial: 6 months \\
\hline
\end{tabular}

Rehabilitation for distal radial fractures in adults (Review) 
Table 4. Assessment of items relating to applicability of trial findings (Continued)

\begin{tabular}{|c|c|c|c|c|c|}
\hline Watt 2000 & $\begin{array}{l}\text { Partial: not fully de- } \\
\text { fined - Colles' frac- } \\
\text { ture, treated with } \\
\text { plaster cast, no "sig- } \\
\text { nificant past his- } \\
\text { tory" }\end{array}$ & $\begin{array}{l}\text { Partial: treatment, } \\
\text { while recorded, was } \\
\text { at the discretion of } \\
\text { the hospital thera- } \\
\text { pists with the con- } \\
\text { tent of the treat- } \\
\text { ment recorded but } \\
\text { not controlled }\end{array}$ & Yes & $\begin{array}{l}\text { Partial: no PROMs, } \\
\text { but clearly a sys- } \\
\text { tematic approach to } \\
\text { data collection for } \\
\text { the few outcomes }\end{array}$ & No: 6 weeks \\
\hline
\end{tabular}

PROM: patient-reported outcome measure

Table 5. Summary of rehabilitation intervention versus no intervention (start: definitive treatment period)

\begin{tabular}{l} 
Occupational or other hand therapy \\
$\begin{array}{ll}\text { PICO } & \begin{array}{l}\text { Population: } 2 \text { trials, (Cooper 2001), } 17 \text { participants; and (Gronlund 1990), } 40 \text { participants. } \\
\text { All with distal radius fracture treated with plaster cast immobilisation }\end{array} \\
& \text { Intervention: occupational or other hand therapy - Cooper 2001: routine therapy for cast } \\
& \text { wear for 4 weeks; Gronlund 1990: occupational therapy single session } \\
\text { Control: instructions for home care at fracture clinic/casualty ward } & \text { Outcomes: DASH, modified Gartland and Werley composite outcome score, grip strength, } \\
\text { pinch grip, range of motion, dexterity, finger movement, complications and cast problems, } \\
\text { referral to hand therapy, use of appliances and home help, oedema, participant satisfaction; } \\
\text { follow-up } 4 \text { weeks (Cooper 2001); } 13 \text { weeks (Gronlund 1990) }\end{array}$ \\
\hline
\end{tabular}

Findings $\quad$ No pooling undertaken due to lack of common outcomes and heterogeneity. Cooper 2001 found better functional and clinical results in the intervention group at four days after removal of plaster cast, with some beneficial effects continuing one month later. Fewer people in the early therapy group met the criteria for attending the post-immobilisation hand therapy classes. Gronlund 1990 found that early "occupational therapy" resulted in significantly better hand function at cast removal but not at 13 weeks for older people with stable fractures treated with plaster casts. Assessment at occupational therapy revealed a need for manual aids, home help and plaster cast adjustment as well as a lack of understanding of the instructions for exercises and advice initially provided to all trial participants

GRADE

Very low quality evidence. Reasons for downgrading according to source of data: -1 level for study limitations (mainly, performance bias; with confounding from a major imbalance in age in Gronlund 1990), -2 levels for serious imprecision (small trials, no pooling)

Comments

Applicability reduced by inadequate follow-up in both trials, and no PROMs in Gronlund 1990 (Table 4)

As well as the small sample size, there are potential problems with confounding in Cooper 2001 due to differences in baseline characteristics and care programmes (there was no information on the numbers requiring longer plaster cast immobilisation). Thus, these promising results need confirmation in a larger sample size, with a longer duration of followup. Small sample size and incomplete assessment of outcome, for instance in the numbers referred for occupational therapy after plaster cast removal, in Gronlund 1990 mean that

Rehabilitation for distal radial fractures in adults (Review) 
there was insufficient evidence to confirm either a lack of longer-term difference in outcome or a short-term advantage of early occupational therapy

\section{Cyclic pneumatic soft tissue compression}

\begin{tabular}{ll}
\hline PICO & $\begin{array}{l}\text { Population: } 1 \text { trial (Challis 2007), } 19 \text { followed up of } 21 \text { participants with distal radius } \\
\text { fracture treated with plaster cast immobilisation } \\
\text { Intervention: cyclic pneumatic soft tissue compression using an inflatable cuff placed under } \\
\text { the plaster cast - applied at home (Table 3) } \\
\text { Control: usual care during immobilisation } \\
\text { Outcomes: grip and pinch strength and range of motion; follow-up } 10 \text { weeks }\end{array}$ \\
\hline Findings & $\begin{array}{l}\text { Intervention group had better and earlier recovery in grip and pinch strength and range of } \\
\text { motion at end of treatment (6 weeks from fracture) and } 10 \text { weeks (4 weeks post-immobili- } \\
\text { sation) }\end{array}$ \\
\hline GRADE & $\begin{array}{l}\text { Very low quality evidence. Downgraded: }-1 \text { study limitations (major baseline imbalance } \\
\text { in gender, age and fracture severity); }-2 \text { serious imprecision (very small pilot trial) }\end{array}$ \\
\hline Comments & $\begin{array}{l}\text { Applicability reduced by incomplete description of study population; no PROMs; inade- } \\
\text { quate length of follow-up (Table 4). } \\
\text { The major differences in the baseline characteristics of the two groups (e.g. } 4 / 10 \text { versus } 0 / \\
9 \text { males) may have contributed to the favourable results for the intervention group }\end{array}$
\end{tabular}

\section{Early digit mobilisation programme during external fixation}

\begin{tabular}{ll} 
PICO & $\begin{array}{l}\text { Population: } 1 \text { trial (Kuo 2013), } 22 \text { participants with distal radius fracture treated with } \\
\text { external fixation } \\
\text { Intervention: early digit mobilisation, including massage and passive stretching (18 ses- } \\
\text { sions) - clinic (Table 3) } \\
\text { Control: usual care during external fixation } \\
\text { Outcomes: Taiwanese version of the MAM-36, grip and pinch strength, range of motion } \\
\text { (workspace), finger dexterity, fracture displacement; follow-up } 12 \text { weeks }\end{array}$ \\
\hline Findings & $\begin{array}{l}\text { There were no significant between-group differences in manual ability (measured using } \\
\text { the Taiwanese version of the MAM-36 at 3, } 7 \text { or } 12 \text { weeks post-fracture, or in grip and } \\
\text { pinch strengths and finger dexterity or fracture displacement at } 12 \text { weeks. Although range } \\
\text { of digit motion, expressed as finger "workspace" and thumb "workspace", was greater in the } \\
\text { intervention group, the clinical importance of this is unclear }\end{array}$ \\
\hline
\end{tabular}

GRADE

Very low quality evidence. Downgraded: -1 level for study limitations (high risk of performance and detection biases), -1 level for imprecision (small trial); -1 level for indirectness (non-validated version of MAM-36, uncertain clinical relevance of workspace outcome measure)

Comments

Applicability reduced by insufficient follow-up (Table 4), but also uncertainty over the clinical relevance of outcome measures

These preliminary findings, if true, do not show an important effect that could warrant implementation of this potentially inconvenient and costly intervention: participants needed 
to attend 3 times a week for 6 weeks; 45 minutes each session

Pulsed electromagnetic field (PEMF)

\begin{tabular}{ll}
\hline PICO & $\begin{array}{l}\text { Population: } 1 \text { trial (Lazovic 2012), } 60 \text { women with distal radius fracture treated with plaster } \\
\text { cast immobilisation } \\
\text { Intervention: pulsed electromagnetic field - } 10 \text { sessions in clinic (Table 3) } \\
\text { Control: no PEMF } \\
\text { Outcomes: PRWE, range of motion, complications, oedema; follow-up } 2 \text { to } 3 \text { days after } \\
\text { cast removal }\end{array}$ \\
\hline Findings & $\begin{array}{l}\text { At } 2 \text { to } 3 \text { days after cast removal, the between-group differences in PRWE pain and activity/ } \\
\text { function scores in favour of PEMF are unlikely to have represented clinically important } \\
\text { differences. Range of motion was better in the PEMF group with clinically borderline } \\
\text { differences in supination, flexion and extension. Although fewer participants of the PEMF } \\
\text { group had complications, the diagnosis of these was provisional. The clinical relevance of } \\
\text { the results of the hand oedema measure, which favoured the PEMF group, is unclear }\end{array}$ \\
\hline
\end{tabular}

GRADE

Very low quality evidence. Downgraded: -2 levels for serious study limitations (quasirandomised and non-blinded trial - high risk of selection and detection biases); -1 level for imprecision (small trial)

Comments

Applicability reduced by incomplete description of intervention and very inadequate length of follow-up (Table 4)

As well as concerns about validity of the findings, the inadequate length of follow-up means the consequences of the preliminary findings are unknown. Potentially inconvenient and costly: participants needed to attend each weekday for 2 weeks; 30 minutes each session

\section{Cross-education (strength training of the non-fractured hand)}

PICO Population: 1 trial (Magnus 2013), 39 followed up 51 participants with distal radius fracture treated with cast immobilisation or surgical repair and cast immobilisation

Intervention: standard rehabilitation plus strength training of opposite hand (cross-education) - at home (Table 3)

Control: standard rehabilitation

Outcomes: PRWE, grip strength and range of motion; follow-up 26 weeks

Findings

Between-group differences in patient-reported function (PWRE) were not significant at the three follow-ups (9, 12 and 26 weeks). The intervention group had better grip strength results at all three follow-ups but the between-group difference was only statistically and clinically significant at 12 weeks. A similar finding in favour of cross-education at 12 weeks applied to range of motion. More people in the cross-education group withdrew from the trial

GRADE

Very low quality evidence. Downgraded: -2 levels for serious study limitations (high risks of performance bias and attrition bias - imbalance in participants lost to follow-up (9/ 27 versus 3/24) and in missing data points; imbalance in numbers operated); -1 level for imprecision (small trial) 
Comments

Applicability reduced by incomplete description of study population; insufficient length of follow-up (Table 4)

Potential confounding from imbalances in numbers who had surgery and numbers who withdrew from the trial casts some doubts on the validity of the trial findings of a potential and interim benefit of the intervention on grip strength and range of motion at 12 weeks. These findings were not confirmed by the PWRE scores and as acknowledged by the trial authors: "More investigations are warranted before changes to clinical practice can be recommended"

DASH: Disability of the Arm, Shoulder, and Hand questionnaire

PEMF: pulsed electromagnetic field

PROM: patient-reported outcome measure

PRWE: Patient-Rated Wrist Evaluation

VAS: visual analogue scale

GRADE downgrading of evidence is shown by number of levels (either -1 level or -2 levels, if serious) downgraded for one of five items: study limitations, imprecision, indirectness, inconsistency (not used) and publication bias (not used)

GRADE Working Group grades of evidence

High quality: Further research is very unlikely to change our confidence in the estimate of effect.

Moderate quality: Further research is likely to have an important impact on our confidence in the estimate of effect and may change the estimate.

Low quality: Further research is very likely to have an important impact on our confidence in the estimate of effect and is likely to change the estimate.

Very low quality: We are very uncertain about the estimate.

Table 6. Summary of rehabilitation intervention versus no intervention (start: post-immobilisation)

\section{Single session of physiotherapy}

\begin{tabular}{ll} 
PICO & $\begin{array}{l}\text { Population: } 1 \text { trial (Kay 2008), } 47 \text { followed up of } 56 \text { participants with distal radius fracture, } \\
\text { after plaster cast immobilisation (15 had pins and cast) } \\
\text { Intervention: single session of physiotherapy, primarily advice and instructions for a home ex- } \\
\text { ercise programme (Table 3) } \\
\text { Control: no intervention } \\
\text { Outcomes: PRWE (pain and function), QuickDASH, grip strength, range of motion, thumb } \\
\text { motion, web space, complications, participant satisfaction with intervention, compliance (just } \\
\text { physiotherapy group), request for physiotherapy; follow-up } 6 \text { weeks }\end{array}$ \\
\hline Findings & $\begin{array}{l}\text { Intervention group had better function (clinically important between-group differences favouring } \\
\text { the intervention in PRWE scores for pain and function, and QuickDASH scores for general, } \\
\text { sports and work functioning). There were no significant between-group differences at six weeks } \\
\text { in grip strength, range of motion or complications. Slightly fewer participants requested more } \\
\text { physiotherapy in the physiotherapy group }\end{array}$ \\
\hline
\end{tabular}
and detection biases; attrition bias - differences in lost to follow-up); -1 level for imprecision (small trial) 
Table 6. Summary of rehabilitation intervention versus no intervention (start: post-immobilisation) (Continued)

Applicability reduced by inadequate length of follow-up (Table 4)
The assessment at baseline and at 3 and 6 weeks follow-up by an independent physiotherapist, who
noted complications, could be considered as part the care package provided to both groups and
thus 'no intervention' is not as stark as it seems. Prior care before and during cast immobilisation
may also have varied and impacted on the results. These are promising yet preliminary results
given the small, short-term follow-up, and single centre setting

\section{Physiotherapy or occupational therapy}

\begin{tabular}{|c|c|}
\hline PICO & $\begin{array}{l}\text { Population: } 4 \text { trials, (Bache 2001), } 75 \text { followed up of } 98 \text { participants; (Christensen 2001), } 30 \\
\text { followed up of } 32 \text { participants; (Maciel 2005), } 33 \text { followed up of } 45 \text { participants; (Wakefield 2000) } \\
\text {, } 66 \text { followed up of } 96 \text { participants. All with distal radius fracture, after plaster cast immobilisation } \\
\text { ( } 7 \text { participants had K-wires and cast in Maciel 2005) } \\
\text { Intervention: Physiotherapy or occupational therapy - Bache 2001: physiotherapy (contents/ } \\
\text { schedule at discretion of therapists); Christensen 2001: occupational therapy ( } 2 \text { x weekly until no } \\
\text { more progress); Maciel 2005: activity focussed physiotherapy' up to } 6 \text { weeks; Wakefield 2000: } \\
\text { physiotherapy (contents/schedule at discretion of therapists). All participants received instructions } \\
\text { for home exercises } \\
\text { Control: instructions for home exercises from therapist; (single session of advice on exercises } \\
\text { from physiotherapist in Maciel 2005) } \\
\text { Outcomes: PWRE, Levine functional analysis score, activities of daily living, modified Gartland } \\
\text { and Werley composite outcome score, grip strength, range of motion, pain, SF-36 (quality of } \\
\text { life), complications, referral to occupational therapy, number of sessions, duration of therapy; } \\
\text { follow-up } 12 \text { weeks (Bache 2001); } 9 \text { months (Christensen 2001); } 24 \text { weeks (Maciel 2005); } 6 \\
\text { months (Wakefield 2000) }\end{array}$ \\
\hline Findings & $\begin{array}{l}\text { No pooling undertaken due to lack of common outcomes and heterogeneity } \\
\text { None of the trials found a clinically significant effect of the routine provision of either occupational } \\
\text { therapy (Christensen 2001), physiotherapy (Bache 2001; Wakefield 2000) or 'activity-focussed' } \\
\text { physiotherapy (Maciel 2005). Participants attended on average } 37 \text { therapy sessions in Christensen } \\
\text { 2001, whereas the median number of sessions or contacts was } 3 \text { in both Bache } 2001 \text { and Wakefield } \\
\text { 2000, and averaged } 4.4 \text { sessions in Maciel 2005. Of the participants in the control groups, none } \\
\text { received additional physiotherapy or occupational therapy in Bache } 2001 \text { and Christensen 2001, } \\
\text { and two received remedial physiotherapy in Wakefield } 2000\end{array}$ \\
\hline
\end{tabular}

GRADE

Low quality evidence (overall). Reasons for downgrading according to source of data: -1 level for study limitations (mainly, performance bias; with attrition bias for Wakefield 2000), -1 level for imprecision (small trials, no pooling)

Comments

Applicability reduced by insufficient description of intervention and insufficient follow-up in Bache 2001 (Table 4)

Applicability considerably reduced by incomplete description of population, intervention and outcomes, no PROMs and insufficient follow-up in Christensen 2001 (Table 4)

Applicability much reduced by insufficient follow-up in Maciel 2005 (Table 4)

Applicability much reduced by incomplete description of intervention, no PROMs and insufficient follow-up in Wakefield 2000 (Table 4)

Although four trials addressed essentially the same issue, namely routine or formal provision of therapy in addition to instructions for home exercises after plaster cast removal, the form of

Rehabilitation for distal radial fractures in adults (Review) 
Table 6. Summary of rehabilitation intervention versus no intervention (start: post-immobilisation) (Continued)

the therapy varied. In particular, the therapy tested by Maciel 2005 was specifically focussed on restoring optimal motor performance of activities that were limited in the individual participants. Three trials (Bache 2001; Christensen 2001; Wakefield 2000) focussed on older people and all four trials excluded those with serious complaints already manifest at cast removal, such as pain (Christensen 2001) or CRPS-1 (Bache 2001; Maciel 2005; Wakefield 2000). Three trials (Bache 2001; Maciel 2005; Wakefield 2000 explicitly selected patients who were able to understand instructions

Individually, none of these trials provide sufficiently robust evidence to confirm the finding of a lack of clinically important differences. All are prey to a type 2 error (false conclusion of no difference). Baseline differences hampered the analysis of the results of Bache 2001, but there was some evidence that the physiotherapy group tended to improve more from a less favourable starting position. The loss to follow up of eight (19.5\%) of the 41 participants who started the trial interventions in Maciel 2005 could also have given rise to important bias. Ultimately, no pooling of data was possible and at best the general agreement in these four studies can only be viewed as weak evidence

\section{Continuous passive motion}

\begin{tabular}{ll} 
PICO & $\begin{array}{l}\text { Population: } 1 \text { trial (Rozencwaig 1996), } 7 \text { participants with distal radius fracture, after external } \\
\text { fixation } \\
\text { Intervention: occupational therapy plus continuous passive motion - at home ( } 4 \text { to } 6 \text { hours/day } \\
\text { for } 1 \text { month) (Table 3) } \\
\text { Control: occupational therapy } \\
\text { Outcomes: time to achieve independent status; follow-up to initial recovery (a few weeks) }\end{array}$ \\
\hline Findings & $\begin{array}{l}\text { The } 3 \text { participants in the intervention group took less time (mean } 12 \text { days) to achieve a completely } \\
\text { independent status than the } 4 \text { control group participants }\end{array}$
\end{tabular}

GRADE

Very low quality evidence. Downgraded: -2 levels for serious study limitations (quasi-randomised and non-blinded trial - high risk of selection and detection biases); -1 level for (serious) imprecision (very small trial)

Comments

Applicability substantially reduced by incomplete description of study population, intervention, outcome measures, no PROMs and insufficient length of follow-up (Table 4).

This is a totally inadequate trial from which no conclusions relating to practice should be drawn

Pulsed electromagnetic field (PEMF)

PICO

Population: 1 trial (Cheing 2005), 83 participants with distal radius fracture, after plaster cast immobilisation

Intervention: pulsed electromagnetic field (PEMF) (with or without ice) - 5 sessions in clinic (Table 3)

Control: sham PEMF (with or without ice)

Outcomes: pain, range of motion, adverse events, oedema; follow-up 5 days

Findings

None of the differences in pain, oedema (volume) and range of motion between the combined PEMF groups and combined sham PEMF groups were clinically important or statistically significant. There were no adverse effects recorded 
Table 6. Summary of rehabilitation intervention versus no intervention (start: post-immobilisation) (Continued)

\begin{tabular}{|c|c|}
\hline GRADE & $\begin{array}{l}\text { Very low quality evidence. Downgraded: }-1 \text { level for study limitations (mainly relating to unclear } \\
\text { risk of selection, performance and detection biases), }-1 \text { level for imprecision (small trial); -1 level } \\
\text { for indirectness (very short follow-up, measured before last session of intervention) }\end{array}$ \\
\hline Comments & $\begin{array}{l}\text { Applicability considerably reduced by lack of PROMs and very inadequate follow-up (Table 4) } \\
\text { The clinical relevance of these results, if true, is uncertain given the premature and very short- } \\
\text { term follow-up. Additionally, confounding could have resulted from baseline imbalances in some } \\
\text { outcome measures. Potentially inconvenient and costly: participants needed to attend each week- } \\
\text { day for } 1 \text { week; } 30 \text { minutes each session }\end{array}$ \\
\hline \multicolumn{2}{|l|}{ Ice } \\
\hline PICO & $\begin{array}{l}\text { Population: } 1 \text { trial (Cheing 2005), } 83 \text { participants with distal radius fracture, after plaster cast } \\
\text { immobilisation } \\
\text { Intervention: ice (with real or sham PEMF) - } 5 \text { sessions in clinic (Table 3) } \\
\text { Control: no ice (with real or sham PEMF) } \\
\text { Outcomes: pain, range of motion, adverse events, oedema; follow-up } 5 \text { days }\end{array}$ \\
\hline Findings & $\begin{array}{l}\text { Statistically significant but clinically unimportant findings of less pain and worse extension results } \\
\text { in the ice treatment group }(s) \text {. None of the differences in oedema (volume) and other range of } \\
\text { motion variables between the combined ice groups and combined control groups were clinically } \\
\text { important or statistically significant. There were no adverse effects recorded }\end{array}$ \\
\hline GRADE & $\begin{array}{l}\text { Very low quality evidence. Downgraded: }-1 \text { level for study limitations (mainly relating to unclear } \\
\text { risk of selection, performance and detection biases), }-1 \text { level for imprecision (small trial); }-1 \text { level } \\
\text { for indirectness (very short follow-up, measured before last session of intervention) }\end{array}$ \\
\hline Comments & $\begin{array}{l}\text { Applicability considerably reduced by lack of PROMs and very inadequate follow-up (Table 4) } \\
\text { The clinical relevance of these results, if true, is uncertain given the premature and very short- } \\
\text { term follow-up. Additionally, confounding could have resulted from baseline imbalances in some } \\
\text { outcome measures. Potentially inconvenient: participants needed to attend each weekday for } 1 \\
\text { week }\end{array}$ \\
\hline
\end{tabular}

Pulsed electromagnetic field (PEMF) plus ice

PICO Population: 1 trial (Cheing 2005), 39 participants with distal radius fracture, after plaster cast immobilisation

Intervention: PEMF plus ice - 5 sessions in clinic (Table 3)

Control: sham PEMF and no ice

Outcomes: pain, range of motion, adverse events, oedema; follow-up 5 days

Findings

Statistically significant but clinically unimportant findings of worse extension but better ulnar deviation results in the intervention group. None of the between-group differences in pain, oedema (volume) and other range of motion variables were clinically important or statistically significant. There were no adverse effects recorded

GRADE

Very low quality evidence. Downgraded: -1 level for study limitations (mainly relating to unclear risk of selection, performance and detection biases), -1 level for imprecision (small trial); -1 level

Rehabilitation for distal radial fractures in adults (Review) 
Table 6. Summary of rehabilitation intervention versus no intervention (start: post-immobilisation) (Continued)

for indirectness (very short follow-up, measured before last session of intervention)

Comments

Applicability considerably reduced by lack of PROMs and very inadequate follow-up (Table 4) The clinical relevance of these results, if true, is uncertain given the premature and very shortterm follow-up. Additionally, confounding could have resulted from baseline imbalances in some outcome measures. Potentially inconvenient and costly: participants needed to attend each weekday for 1 week; 30 minutes each session

\section{Passive mobilisation}

PICO
Population: 2 trials (Kay 2000), 39 followed up of 40 participants; and (Taylor 1994), 30
participants. All with distal radius fracture, post-plaster cast immobilisation
Intervention: passive mobilisation - Kay 2000: 9 sessions over 6 weeks; Taylor $1994: 5$ minutes
during twice weekly physiotherapy (Table 3)
Control: Kay 2000: all attended physiotherapy session for advice \& instructions; and assessment
at 3 and 6 weeks, more at physiotherapist's discretion; Taylor 1994: sham (soft tissue massage)
and physiotherapy
Outcomes: pain \& functional disability, grip strength, range of motion, web space angle, com-
plications, number of physiotherapy sessions, time to discharge, costs; follow-up 6 weeks (Kay
2000); until discharge (mean 26 days) (Taylor 1994)
$\begin{aligned} & \text { No pooling undertaken due to lack of common outcomes and heterogeneity. Both trials found } \\ & \text { no significant between-group differences in short-term outcome; this was primarily active wrist } \\ & \text { extension in Taylor } 1994 \text {. There were no between-group differences in complications. Participants } \\ & \text { allocated passive mobilisation received on average six more treatments than those in the control } \\ & \text { group in Kay } 2000 \text {, which estimated additional passive mobilisation to be nearly three times as } \\ & \text { expensive as a regimen of advice and exercises alone. There was little difference }(<1 \text { session) in } \\ & \text { numbers of sessions between the two groups in Taylor } 1994\end{aligned}$

GRADE

Very low quality evidence. Reasons for downgrading according to source of data: -1 level for study limitations (mainly, performance bias; or selection and performance biases), -2 levels for serious imprecision (small trials, no pooling)

Comments

Applicability much reduced by lack of PROMs and inadequate follow-up (Table 4)

Neither trial was sufficient to take their lack of significant differences as evidence of no effect. Passive mobilisation delivered as in Kay 2000 was much more costly. The soft tissue massage control of Taylor 1994 cannot be considered a 'placebo'; it may still have a therapeutic role, for instance in redistributing tissue fluid, and thus potentially diminish any effects of passive mobilisation

\section{Intermittent pneumatic compression}

PICO
Population: 1 trial (Svensson 1993), 31 followed up of 43 participants with distal radius fracture,
referred for rehabilitation after plaster cast immobilisation
Intervention: intermittent pneumatic compression (20 minutes) before occupational therapy -
minimum $3 \mathrm{x}$ weekly for 3 weeks (Table 3)
Control: occupational therapy only
Outcomes: use of hand (VAS), pain, range of motion, complications, oedema, number of sessions

Rehabilitation for distal radial fractures in adults (Review) 


\begin{tabular}{ll}
\hline Findings & $\begin{array}{l}\text { There were no data to examine the report of improved wrist extension and tendencies for im- } \\
\text { provements in other outcomes favouring the intervention group. Only a few participants in each } \\
\text { group (numbers not stated) were considered to require further occupational therapy after three } \\
\text { weeks }\end{array}$ \\
\hline GRADE & $\begin{array}{l}\text { Very low quality evidence. Downgraded: }-2 \text { levels for serious study limitations (performance } \\
\text { and detection biases, attrition bias - missing results for } 12 \text { participants), }-1 \text { level for imprecision } \\
\text { (small trial) }\end{array}$ \\
\hline Comments & $\begin{array}{l}\text { Applicability reduced by no PROMs and insufficient follow-up (Table } 4) \\
\text { The evidence from this small, inadequately reported and flawed trial is insufficient to inform } \\
\text { practice. Potentially inconvenient and costly: participants needed to stay } 20 \text { minutes extra each } \\
\text { of } 9 \text { sessions }\end{array}$ \\
\hline
\end{tabular}

Ultrasound

PICO

Population: 1 trial (Basso 1998), 38 participants with distal radius fracture, after plaster cast immobilisation

Intervention: ultrasound - 5 minutes (Table 3)

Control: sham ultrasound

Outcomes: range of motion, referral for physiotherapy

Findings

There was no between-group difference in wrist motion but fewer ultrasound participants were referred for physiotherapy at eight weeks $(2 / 19$ versus $8 / 19 ; \mathrm{P}=0.05)$

GRADE

Very low quality evidence. Downgraded: -1 level for study limitations (quasi-randomised, thus high risk of selection bias), -1 level for imprecision (small trial); -1 level for indirectness (relating to outcome)

Comments

Applicability considerably reduced by incomplete details on intervention, limited outcomes, no PROMs and insufficient follow-up (Table 4)

Baseline and care programme comparability were not confirmed in this small quasi-randomised trial; the very limited evidence is insufficient to inform practice. The underlying mechanism for the intervention is not established

\section{Whirlpool}

PICO

Population: 1 trial ( Toomey 1986), 24 participants with distal radius fracture, referred to Physical Medicine Department after plaster cast immobilisation

Intervention: whirlpool bath immersion for 15 minutes prior to exercises - 12 sessions, twice weekly (Table 3)

Control: towel only

Outcomes: pain, grip strength, range of motion, oedema; follow-up 6 weeks 


\begin{tabular}{|c|c|}
\hline GRADE & $\begin{array}{l}\text { Very low quality evidence. Downgraded: }-1 \text { level for study limitations (performance bias, unclear } \\
\text { risk of several other biases), }-2 \text { levels for serious imprecision (very small trial) }\end{array}$ \\
\hline Comments & $\begin{array}{l}\text { Applicability reduced by no PROMs and insufficient follow-up (Table 4) } \\
\text { Whirlpool bath immersion prior to exercises was reported as common in Canadian physiotherapy } \\
\text { departments in the early 1980s. The inadequate sample size, unresolved questions on participant } \\
\text { numbers, and a variable and short-term follow up amount to potentially flawed and insufficient } \\
\text { evidence for this modality. Potentially inconvenient and costly: participants who need to stay } 15 \\
\text { minutes extra each of } 12 \text { sessions }\end{array}$ \\
\hline \multicolumn{2}{|c|}{ Dynamic wrist extension splint } \\
\hline PICO & $\begin{array}{l}\text { Population: } 1 \text { trial (Jongs 2012), } 40 \text { participants with wrist contracture who had been referred } \\
\text { to physiotherapy at least } 10 \text { weeks from their conservatively or surgically treated fracture } \\
\text { Intervention: routine care plus dynamic wrist extension splints, worn up to six hours at home } \\
\text { on a daily basis for eight weeks (Table } 3 \text { ) } \\
\text { Control: routine care (exercises and advice) } \\
\text { Outcomes: PRWE, range of motion, Canadian Occupational Performance Measure (COPM), } \\
\text { adverse events, splint repair, adherence; follow-up } 12 \text { weeks }\end{array}$ \\
\hline Findings & $\begin{array}{l}\text { There were no clinically or statistically between-group differences in PRWE, range of motion } \\
\text { and COPM at } 8 \text { or } 12 \text { weeks. There were two adverse events, which resolved after modification } \\
\text { of the splint, and two other splints required repair. Thus problems were encountered in a quarter } \\
\text { of the group allocated splints. Compliance with the splint regimen was poor }\end{array}$ \\
\hline GRADE & $\begin{array}{l}\text { Very low quality evidence. Downgraded: }-1 \text { level for study limitations (performance and detec- } \\
\text { tion biases), }-1 \text { level for imprecision (small trial), }-1 \text { level for indirectness (population is subgroup } \\
\text { of distal radius patients) }\end{array}$ \\
\hline Comments & $\begin{array}{l}\text { Applicability reduced by insufficient follow-up (Table 4) } \\
\text { There was poor adherence to the intended six hour daily application for the splint, with complaints } \\
\text { by some participants of the limitations that the splints imposed on day-to-day activities. Thus } \\
\text { this is an inconvenient and demanding intervention. The trial authors' conclusion that dynamic } \\
\text { splints are unlikely to be therapeutic are consistent with the findings of a lack of effectiveness for } \\
\text { 'stretch', including that administered via splints, in Katalinic } 2010\end{array}$ \\
\hline
\end{tabular}


DASH: Disability of the Arm, Shoulder, and Hand questionnaire

PROM: patient-reported outcome measure

PRWE: Patient-Rated Wrist Evaluation

VAS: visual analogue scale

GRADE downgrading of evidence is shown by number of levels (either -1 level or -2 levels, if serious) downgraded for one of five items: study limitations, imprecision, indirectness, inconsistency (not used) and publication bias (not used)

\section{GRADE Working Group grades of evidence}

High quality: Further research is very unlikely to change our confidence in the estimate of effect.

Moderate quality: Further research is likely to have an important impact on our confidence in the estimate of effect and may change the estimate.

Low quality: Further research is very likely to have an important impact on our confidence in the estimate of effect and is likely to change the estimate.

Very low quality: We are very uncertain about the estimate.

Table 7. Summary of one rehabilitation intervention versus another rehabilitation intervention (start: post-immobilisation)

Physiotherapy versus instructions for home exercises by an orthopaedic surgeon

PICO

Findings

GRADE

Comments

Pulsed electromagnetic field (PEMF) versus ice
Population: 1 trial (Watt 2000), 16 followed up of 18 participants with distal radius fracture, after plaster cast immobilisation

Intervention: routine physiotherapy (Table 3)

Control: home exercise sheet and simple home instructions given at outpatients by orthopaedic surgeon/registrar

Outcomes: grip strength and range of motion (wrist extension), number of physiotherapy sessions; follow-up 6 weeks

Trial found significantly better grip strength and wrist extension at six weeks in participants given physiotherapy. Physiotherapy group participants attended an average of five sessions

Very low quality evidence. Downgraded: -1 level for study limitations (performance and detection biases); -2 levels for serious imprecision (very small trial, little data)

Applicability much reduced by incomplete descriptions of study population and physiotherapy; limited outcomes and no PROMs, and inadequate length of follow-up (Table 4)

These promising yet preliminary results need confirmation with larger numbers, longer-term follow up, and a more comprehensive appraisal of outcome, and replication in different settings
Population: 1 trial (Cheing 2005), 44 participants with distal radius fracture, after plaster cast immobilisation

Intervention: PEMF plus ice - 5 sessions in clinic (Table 3)

Control: ice

Outcomes: pain, range of motion, adverse events, oedema; followup 5 days 
Table 7. Summary of one rehabilitation intervention versus another rehabilitation intervention (start: post-immobilisation) (Continued)

Findings

Statistically significant but clinically marginal findings of worse pain but better extension results in the PEMF group. However, these are consistent with similar differences in baseline values between the two group. None of the between-group differences were clinically important or statistically significant. There were no adverse effects recorded

GRADE

Very low quality evidence. Downgraded: - 1 level for study limitations (mainly relating to unclear risk of selection, performance and detection biases), -1 level for imprecision (small trial); - 1 level for indirectness (very short follow-up, measured before last session of intervention)

Comments

Applicability considerably reduced by lack of PROMs and very inadequate follow-up (Table 4)

Ideally, the effects of the two interventions should be established before a comparison of their relative effects. This is not the case so far. Serious deficiencies in the measurement of outcome and baseline imbalances in the trial testing this comparison mean that no conclusions can be drawn on the relative effectiveness of PEMF versus ice in treating pain, swelling and stiffness after plaster cast removal

Modified “manual edema mobilization” (MEM) versus 'traditional' oedema treatment

PICO

Population: 1 trial (Knygsand-Roenhoej 2011), 29 followed up of 30 participants with distal radius fracture who had subacute oedema 4 to 10 weeks post-trauma/surgery (plaster cast (11); surgery (18: external or internal fixation)) and a $60 \mathrm{~mL}$ or more in volume difference between the upper extremities

Intervention: Modified “manual edema mobilization" (MEM) (Table 3)

Control: 'Traditional oedema treatment': elevation, compression, and functional training

Outcomes: Activities of daily living, Canadian Occupational Performance Measure, complication, oedema, number of occupational therapy sessions; follow-up 6 months

Findings

The trial found no clinically important difference in measures of function up to nine weeks or pain at 9 or 26 weeks between these two methods of treating subacute oedema after treatment for distal radius fracture. Two complications were reported in the MEM group

GRADE

Very low quality evidence. Downgraded: -1 level for study limitations (high risk of performance and detection biases); - 1 level for imprecision (small trial); -1 level for indirectness (population is subgroup of distal radius patients; also could depend on clinicians providing treatment)

Rehabilitation for distal radial fractures in adults (Review)

Copyright () 2015 The Cochrane Collaboration. Published by John Wiley \& Sons, Ltd. 
Table 7. Summary of one rehabilitation intervention versus another rehabilitation intervention (start: post-immobilisation) (Continued)

Comments Applicability reduced by insufficient length of follow-up (Table
4). Additionally, the results apply to the patient subgroup with
subacute oedema
Although the traditional therapy group tended to receive more
treatment sessions, the reasons for this may be other than the treat-
ment itself.These include differences in the two clinicians pro-
viding treatment, a slightly higher level of oedema in the tradi-
tional group at baseline and other differences in the interventions.
Oedema is often associated with hand and wrist trauma and so
any future research to test the use of MEM could be expanded to
include other injuries

PROM: patient-reported outcome measure

GRADE downgrading of evidence is shown by number of levels (either -1 level or -2 levels, if serious) downgraded for one of five items: study limitations, imprecision, indirectness, inconsistency (not used) and publication bias (not used)

GRADE Working Group grades of evidence

High quality: Further research is very unlikely to change our confidence in the estimate of effect.

Moderate quality: Further research is likely to have an important impact on our confidence in the estimate of effect and may change the estimate.

Low quality: Further research is very likely to have an important impact on our confidence in the estimate of effect and is likely to change the estimate.

Very low quality: We are very uncertain about the estimate.

Table 8. Summary of any method (context) of delivering or providing rehabilitation interventions versus any other method of delivering or providing rehabilitation interventions (start: definitive treatment period)

Exercise therapy supervised by a physiotherapist versus instructions for the same exercises given by an orthopaedic surgeon

PICO

Findings
Population: 1 trial (Pasila 1974), 96 followed up of 135 participants with distal radius fracture, after plaster cast immobilisation Intervention: exercise therapy supervised by a physiotherapist ( Table 3)

Control: instructions for the same exercises given by an orthopaedic surgeon

Outcomes: grip strength and range of motion, time to return to work, visits to physiotherapy department; follow-up 12 weeks

Trial found no significant between group differences in strength or range of motion, time to return to work. The physiotherapy group participants visited the physical medicine department an average of four times

GRADE

Very low quality evidence. Downgraded: -2 levels for serious study limitations (performance and detection biases, attrition bias (29\% losses), selective outcome reporting bias); -1 level for imprecision (small trial) 
Table 8. Summary of any method (context) of delivering or providing rehabilitation interventions versus any other method of delivering or providing rehabilitation interventions (start: definitive treatment period) (Continued)

Comments

PROM: patient-reported outcome measure

GRADE downgrading of evidence is shown by number of levels (either -1 level or -2 levels, if serious) downgraded for one of five items: study limitations, imprecision, indirectness, inconsistency (not used) and publication bias (not used)

GRADE Working Group grades of evidence

High quality: Further research is very unlikely to change our confidence in the estimate of effect.

Moderate quality: Further research is likely to have an important impact on our confidence in the estimate of effect and may change the estimate.

Low quality: Further research is very likely to have an important impact on our confidence in the estimate of effect and is likely to change the estimate.

Very low quality: We are very uncertain about the estimate.

Table 9. Summary of any method (context) of delivering or providing rehabilitation interventions versus any other method of delivering or providing rehabilitation interventions (start: post-immobilisation)

Physiotherapy versus a home exercise programme

$\begin{array}{ll}\text { PICO } & \begin{array}{l}\text { Population: } 1 \text { trial (Bighea 2013), } 20 \text { participants with osteoporotic wrist } \\ \text { fracture, after plaster cast immobilisation }\end{array} \\ & \text { Intervention: physiotherapy galvanic bath, } 30 \text { minutes exercise each session } \\ \text { programme; } 5 \text { xeek for } 4 \text { weeks (Table 3) } & \text { Control: home exercise programme, instructions for } 15 \text { minutes } 2 \times \text { daily } \\ \text { sheet } & \text { Outcomes: PRWE, range of motion; follow-up } 8 \text { weeks }\end{array}$

Findings

The trial reported better PRWE scores and greater increases in extension and flexion in the physiotherapy group after four weeks

GRADE

Very low quality evidence. Downgraded: -1 level for study limitations (performance and detection biases, selective outcome reporting bias); -2 levels for serious imprecision (very small trial, little data)

Comments

Rehabilitation for distal radial fractures in adults (Review)

Copyright () 2015 The Cochrane Collaboration. Published by John Wiley \& Sons, Ltd.
Applicability much reduced by incomplete descriptions of study population, interventions and outcomes, and inadequate length of follow-up (Table 4) These preliminary results need confirmation with larger numbers, longerterm follow up, and a more comprehensive appraisal of outcome, and replication in different settings
Applicability much reduced by incomplete descriptions of study and inadequate length of follow-up (Table 4).

up, and inadequate sample size of Pasila 1974 mean that the lack of significant differences in various measures of recovery between the two participant groups cannot be considered as reliable evidence. Noteworthy is that this was a comparatively young population, over two-thirds of whom were under 40 years old, and thus not generally representative population and physiotherapy; limited outcomes and no PROMs,

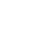


Table 9. Summary of any method (context) of delivering or providing rehabilitation interventions versus any other method of delivering or providing rehabilitation interventions (start: post-immobilisation) (Continued)

\section{Physiotherapy or occupational therapy versus progressive home exercise programme after volar plate fixation}

$\begin{array}{ll}\text { PICO } & \begin{array}{l}\text { Population: } 2 \text { trials (Krischak 2009), } 46 \text { followed up of } 48 \text { participants; and } \\ \text { (Souer 2011), } 76 \text { followed up of 94 participants. All with distal radius fracture, } \\ \text { after volar plate fixation }\end{array} \\ \text { Intervention: physiotherapy or occupational therapy: } 12 \text { sessions 20-30 min- } \\ \text { utes (Krischak 2009); at discretion of therapist (Souer 2011) (Table 3) } \\ \text { Control: progressive home exercise programme } \\ \text { Outcomes: PRWE, DASH, Mayo wrist scores, pain, grip and pinch strength } \\ \text { and range of motion, complications, change of treatment, compliance, costs; } \\ \text { follow-up } 6 \text { weeks (Krischak 2009), } 6 \text { months (Souer 2011) }\end{array}$

Findings

Both trials in this category found some evidence in favour of a structured programme of home exercises preceded by instructions or coaching compared with supervised therapy after surgery. At the end of the six-weeks treatment programme, Krischak 2009 found physiotherapy resulted in significantly poorer self reported function as well as objective measures of function. Although finding no difference between the two groups in self reported function at three or six months follow-up, Souer 2011 reported poorer grip strength at three months and poorer wrist mobility at six months in the occupational therapy group compared with independent exercise group

GRADE

Low quality evidence. Downgraded: -1 level for study limitations (performance and detection biases); -1 level for imprecision (small trials, no pooling)

Comments

Applicability reduced by incomplete descriptions of physiotherapy/occupational therapy; and insufficient length of follow-up (Table 4)

A marked difference in the duration of exercises was explicit in Krischak 2009, supervised therapy amounted to 40 to 60 minutes per week in contrast to 280 minutes of home exercises. It is unclear but seems unlikely from the accounts of both trials that instructions and encouragement for home exercises were provided in the supervised therapy group. If so, this reduces the applicability given that instructions for home exercises are typically part of usual care. It should be noted that even if independent exercises using these rigorous schemes is enough for most patients after volar plate fixation, this low quality evidence does not rule out a role for supervised therapy. The latter though is potentially inconvenient and more costly: in Krischak 2009, participants needed to attend 12 sessions in 6 weeks

\section{Accelerated versus usual rehabilitation after volar plate fixation}

Population: 1 trial (Brehmer 2014), 63 of 81 participants with distal radius fracture after volar plate fixation

Intervention: accelerated (started at two weeks) rehabilitation (Table 3)

Control: usual rehabilitation (started at six weeks)

Outcomes: DASH scores, grip strength, palmar pinch strength, range of motion, adverse events and splint repair, fracture alignment and healing; 
Table 9. Summary of any method (context) of delivering or providing rehabilitation interventions versus any other method of delivering or providing rehabilitation interventions (start: post-immobilisation) (Continued)

follow-up 6 months

Findings

Although DASH scores favoured accelerated rehabilitation at 8 and 12 weeks and six months, the effect size dropped over time and the confidence intervals did not include the MCID in the two later follow-up times. Grip strength at three and six months was also greater in the accelerated group compared with the usual group, with a significant difference showing at six months. The results for range of motion results favoured the home exercises group but were not clinically important. There were few complications in both groups, and no loss of alignment

GRADE

Very low quality evidence. Downgraded: -2 levels for study limitations (high risk of performance and detection biases; and attrition bias); -1 level for imprecision (small trial)

Comments

Applicability reduced by incomplete description of population and insufficient length of follow-up (Table 4).

The early clinical benefit shown by better DASH results of accelerated rehabilitation did not persist at three and six months. There were few complications and none, such as loss of alignment, that conflicted with either intervention. Although the focus was on rehabilitation for this review, participants in the usual group were scheduled to stop using their removable splints three weeks after those in the accelerated group. It is also not clear if any advice was given to the participants in the usual group to engage in at least range of motion exercises before the start of the strengthening protocol

DASH: Disability of the Arm, Shoulder, and Hand questionnaire

MCID: minimal clinically important difference

PRWE: Patient-Rated Wrist Evaluation

GRADE downgrading of evidence is shown by number of levels (either -1 level or -2 levels, if serious) downgraded for one of five items: study limitations, imprecision, indirectness, inconsistency (not used) and publication bias (not used)

GRADE Working Group grades of evidence

High quality: Further research is very unlikely to change our confidence in the estimate of effect.

Moderate quality: Further research is likely to have an important impact on our confidence in the estimate of effect and may change the estimate.

Low quality: Further research is very likely to have an important impact on our confidence in the estimate of effect and is likely to change the estimate.

Very low quality: We are very uncertain about the estimate. 


\section{A P P E N D I C E S}

\section{Appendix I. Search strategies}

\section{CENTRAL (Wiley Online Library) (2014 Issue 12)}

\#1 MeSH descriptor: [Radius Fractures] explode all trees (300)

\#2 MeSH descriptor: [Wrist Injuries] this term only and with qualifier(s): [Rehabilitation - RH] (9)

\#3 (radius or radial or wrist or colles or smith*) near/3 fracture*:ti,ab,kw (Word variations have been searched) (808)

\#4 \#1 or \#2 or \#3 (809)

\#5 MeSH descriptor: [Rehabilitation] explode all trees (15453)

\#6 MeSH descriptor: [Rehabilitation Nursing] this term only (45)

\#7 Any MeSH descriptor with qualifier(s): [Rehabilitation - RH] (13786)

\#8 MeSH descriptor: [Physical Therapy Modalities] explode all trees (16091)

\#9 MeSH descriptor: [Exercise] explode all trees (14093)

\#10 MeSH descriptor: [Ultrasonic Therapy] explode all trees (752)

\#11 MeSH descriptor: [Cryotherapy] this term only (469)

\#12 MeSH descriptor: [Transcutaneous Electric Nerve Stimulation] this term only (742)

\#13 MeSH descriptor: [Patient Education as Topic] this term only (6619)

\#14 MeSH descriptor: [Postoperative Care] this term only (3869)

\#15 MeSH descriptor: [Recovery of Function] this term only (3080)

\#16 rehabilitat* or exercis* or physiotherap* or therap* or training or mobili* or educat*:ti,ab,kw (Word variations have been searched) (326598)

\#17 \{or \#5-\#16\} (337547)

\#18 \#4 and \#17 (297)

\section{MEDLINE (Ovid Online) (January 2005 to January 2015)}

1 exp Radius Fractures/ (7432)

2 Wrist Injuries/rh (143)

3 ((radius or radial or wrist or colles or smith*2) adj3 fracture*).tw. (6093)

4 or/1-3 (9701)

5 rehabilitation/ or "activities of daily living"/ or exp exercise therapy/ or occupational therapy/or recreation therapy/or rehabilitation, vocational/ (111537)

6 Rehabilitation Nursing/ (1149)

7 rehabilitation.fs. (166443)

8 exp Physical Therapy Modalities/ (126395)

9 exercise/ or muscle stretching exercises/ or plyometric exercise/ or resistance training/ or swimming/ (84477)

10 Ultrasonic Therapy/ (8099)

11 Cryotherapy/ (3776)

12 Transcutaneous Electric Nerve Stimulation/ (3432)

13 Patient Education as Topic/ (70820)

14 Postoperative Care/ (52195)

15 "Recovery of Function"/ (32301)

16 (rehabilitat* or exercis* or physiotherap* or therap* or training or mobili* or educat*).tw. (2782529)

17 or/5-16 (3081123)

18 and/4,17 (2273)

19 randomized controlled trial.pt. (381164)

20 controlled clinical trial.pt. (88412)

21 randomized.ab. (305106)

22 placebo.ab. (156743)

23 drug therapy.fs. (1723534)

Rehabilitation for distal radial fractures in adults (Review)

Copyright () 2015 The Cochrane Collaboration. Published by John Wiley \& Sons, Ltd. 
24 randomly.ab. (221425)

25 trial.ab. (314777)

26 groups.ab. (1400784)

27 or/19-26 (3418450)

28 exp animals/ not humans.sh. (3967499)

2927 not 28 (2934092)

30 and/18,29 (482)

31 (2005* $^{*}$ or $2006^{*}$ or $2007^{*}$ or $2008^{*}$ or $2009^{*}$ or $2010^{*}$ or $2011^{*}$ or $2012^{*}$ or $2013^{*}$ or $2014^{*}$ or $\left.2015^{*}\right)$.ed. (8543954)

3230 and 31 (304)

\section{Embase (Ovid Online) (January 2005 to January 2015)}

1 Radius Fracture/ or Colles Fracture/ (7965)

2 wrist injury/rh [Rehabilitation] (126)

3 ((radius or radial or wrist or colles or smith*2) adj3 fracture*).tw. (6911)

4 or/1-3 (10574)

5 rehabilitation/ or athletic rehabilitation/ or community based rehabilitation/ or functional assessment/ or functional training/ or geriatric rehabilitation/ or home rehabilitation/ or muscle training/ or occupational therapy/ or recreational therapy/or self report/ or sensorimotor integration/ or vocational rehabilitation/ (196875)

6 rehabilitation nursing/ (1106)

7 rh.fs. (128687)

8 rehabilitation care/ (9937)

9 physiotherapy/ or home physiotherapy/ or joint mobilization/ (54682)

10 exp Exercise/ (213633)

11 exp Kinesiotherapy/ (48517)

12 ultrasound therapy/ (7211)

13 cryotherapy/ (12627)

14 transcutaneous nerve stimulation/ (5772)

15 patient education/ or health education/ (162389)

16 postoperative care/ (66810)

17 convalescence/ (34403)

18 daily life activity/ (57417)

19 or/5-18 (850149)

204 and 19 (1460)

21 Randomized controlled trial/ (355791)

22 Clinical trial/ (837351)

23 controlled clinical trial/ (389534)

24 Randomization/ (64178)

25 single blind procedure/ (19230)

26 double blind procedure/ (116818)

27 crossover procedure/ (40987)

28 placebo/ (249956)

29 prospective study/ (270083)

30 ((clinical or controlled or comparative or placebo or prospective $\$$ or randomi\#ed) adj3 (trial or study)).tw. (783742)

31 (random $\$$ adj7 (allocat $\$$ or allot $\$$ or assign $\$$ or basis $\$$ or divid $\$$ or order $\$$ )).tw. (193264)

32 ((singl\$ or doubl\$ or trebl\$ or tripl\$) adj7 (blind\$ or mask\$)).tw. (165549)

33 (cross?over\$ or (cross adj1 over\$)).tw. (71236)

34 ((allocat* or allot* or assign* or divid*) adj3 (condition* or experiment* or intervention* or treatment* or therap* or control* or group $\left.\left.{ }^{*}\right)\right)$.tw. (254332)

35 RCT.tw. (15484)

36 or/21-35 (1952675)

37 Case Study/ or Abstract Report/ or Letter/ (939297)

3836 not 37 (1913454)

Rehabilitation for distal radial fractures in adults (Review)

Copyright $\odot 2015$ The Cochrane Collaboration. Published by John Wiley \& Sons, Ltd. 
39 (exp Animal/ or Animal.hw. or Nonhuman/) not (exp Human/ or Human cell/ or (human or humans).ti.) (5161835)

4038 not $39(1746806)$

4120 and $40(324)$

$42\left(2005^{*}\right.$ or $2006^{*}$ or $2007^{*}$ or $2008^{*}$ or $2009^{*}$ or $2010^{*}$ or $2011^{*}$ or $2012^{*}$ or $2013^{*}$ or $2014^{*}$ or $\left.2015^{*}\right) . e m .(10634718)$

4341 and $42(223)$

\section{CINAHL (Ebsco) (January 2005 to January 20 I5)}

S1 (MH "Radius Fractures") $(1,419)$

S2 (MH "Wrist Injuries/RH”) (49)

S3 TI ( (radius or radial or wrist or colles or smith*) n3 fracture*) OR AB ( (radius or radial or wrist or colles or smith*) n3 fracture*) $(1,478)$

S4 S1 OR S2 OR S3 ( 2,018)

S5 (MH "Rehabilitation+") $(185,976)$

S6 (MH "Rehabilitation Nursing") (2,168)

S7 MW rehabilitation $(100,704)$

S8 (MH “Exercise+") (66,040)

S9 (MH "Ultrasonic Therapy") (1,641)

S10 (MH "Transcutaneous Electric Nerve Stimulation”) (1,385)

S11 (MH "Health Education") OR (MH "Patient Education") $(65,969)$

S12 (MH "Postoperative Care") (12,604)

S13 (MH "Functional Assessment") (12,049)

S14 TI ( rehabilitat* or exercis* or physiotherap* or therap* or training or mobili* or educat*) OR AB ( rehabilitat* or exercis* or physiotherap* or therap* or training or mobili* or educat* $)(578,581)$

S15 S5 OR S6 OR S7 OR S8 OR S9 OR S10 OR S11 OR S12 OR S13 OR S14 $(783,883)$

S16 S4 AND S15 (501)

S17 (MH "Clinical Trials+") $(181,087)$

S18 (MH “Evaluation Research+”) (20,901)

S19 (MH “Comparative Studies") (79,083)

S20 (MH “Crossover Design”) (12,339)

S21 PT Clinical Trial (76,893)

S22 (MH "Random Assignment") (38,220)

S23 S17 or S18 or S19 or S20 or S21 or S22 $(286,569)$

S24 TX ((clinical or controlled or comparative or placebo or prospective or randomi?ed) and (trial or study)) $(514,756)$

S25 TX (random* and (allocat* or allot* or assign* or basis* or divid* or order*)) $(68,846)$

S26 TX ((singl* or doubl* or trebl* or tripl*) and (blind* or mask $\left.\left.{ }^{*}\right)\right)(760,350)$

S27 TX ( crossover* or 'cross over' ) or TX cross n1 over $(15,399)$

S28 TX ((allocat* or allot* or assign* or divid*) and (condition* or experiment* or intervention* or treatment* or therap* or control* or group $\left.\left.{ }^{*}\right)\right)(86,751)$

S29 S24 or S25 or S26 or S27 or S28 $(1,176,873)$

S30 S23 or S29 $(1,246,156)$

S31 S16 AND S30 (276)

S32 EM 2005 OR EM 2006 OR EM 2007 OR EM 2008 OR EM 2009 OR EM 2010 OR EM 2011 OR EM 2012 OR EM 2013 OR EM 2014 OR EM 2015 (3,072,720)

S33 S31 AND S32 (195)

\section{AMED (Ovid Online) (January 2005 to January 20 I5)}

1 exp radius fractures/ (75)

2 wrist injuries/ (102)

3 ((radius or radial or wrist or colles or smith*2) adj3 fracture*).tw. (181)

41 or 2 or $3(254)$

5 randomized controlled trial.pt. (3113)

Rehabilitation for distal radial fractures in adults (Review)

Copyright $\odot 2015$ The Cochrane Collaboration. Published by John Wiley \& Sons, Ltd. 
6 controlled clinical trial.pt. (70)

7 Randomized Controlled Trials/ (1692)

8 Random Allocation/ (312)

9 Double-Blind Method/ (532)

10 or/5-9 (5440)

11 exp Animals/ not Humans/ (7987)

1210 not $11(5410)$

13 clinical trial.pt. (1165)

14 exp Clinical trials/ (3427)

15 (clinic\$ adj25 trial\$).tw. (6030)

16 ((singl\$ or doubl\$ or trebl\$ or tripl\$) adj (mask\$ or blind $\$))$.tw. (2413)

17 Placebos/ (556)

18 placebo\$.tw. (2705)

19 random\$.tw. (14706)

20 exp Research design/ (18130)

21 (latin adj square).tw. (24)

22 or/13-21 (32307)

2322 not $11(31722)$

2423 not 12 (26465)

254 and 24 (35)

$26\left(2005^{*}\right.$ or $2006^{*}$ or $2007^{*}$ or $2008^{*}$ or $2009^{*}$ or $2010^{*}$ or $2011^{*}$ or $2012^{*}$ or $2013^{*}$ or $2014^{*}$ or $\left.2015^{*}\right)$.up. (110554)

2725 and 26 (11)

\section{PEDro (January 2005 to February 2012)}

(http://www.pedro.org.au/)

Body Part: hand or wrist AND Title Only: fracture AND new records added since: 12/01/2005 (49)

\section{LILACS (Bireme) (January 2005 to January 2015)}

\section{5 to 2013}

wrist and fracture (51); radius and fracture (122); distal and radial and fracture (29)

2013 to 2015

(tw:((radius OR radial OR rádio OR radio) AND fractur\$)) AND (tw:((PT:“randomized controlled trial” OR PT:"controlled clinical trial” OR PT:"multicenter study" OR MH:"randomized controlled trials as topic" OR MH:"controlled clinical trials as topic" OR MH: "multicenter studies as topic" OR MH:"random allocation” OR MH:“double-blind method" OR MH:"single-blind method") OR ((ensaio\$ OR ensayo\$ OR trial\$) AND (azar OR acaso OR placebo OR control\$ OR aleat\$ OR random\$ OR enmascarado\$ OR simpleciego OR ((simple\$ OR single OR duplo\$ OR doble\$ OR double\$) AND (cego OR ciego OR blind OR mask))) AND clinic\$)) AND NOT (MH:animals OR MH:rabbits OR MH:rats OR MH:primates OR MH:dogs OR MH:cats OR MH:swine OR PT:“in vitro") )) AND (tw:(DA:2013\$ OR DA:2014\$ OR DA:2015\$)) (106)

\section{OTseeker (January 2005 to February 2013)}

(www.otseeker.com)

Injury Management Search: keywords: radius or radial or colles or wrist: (52)

\section{WHO ICTRP (February 20I5)}

1. colles and fractur* $(15$ records for 14 trials)

2. wrist and fractur* $(67$ records for 65 trials)

3. distal and radi* and fractur* (158 records for 146 trials) 


\section{ClinicalTrials.gov (February 2015)}

1. (radial or radius) AND fracture (22 records)

2. wrist AND fracture (112 records)

3. colles AND fracture (18 records)

\section{Bone and Joint Journal Orthopaedic Proceedings (June 20 I5)}

Title "distal radius fract*" and full text or abstract or title "random*" limited to Orthopaedic Proceedings (28 records) Title "distal radial fract*" and full text or abstract or title "random*" limited to Orthopaedic Proceedings (7 records) Title "wrist fract*" and full text or abstract or title "random*” limited to Orthopaedic Proceedings (4 records)

\section{WHAT'S NEW}

Last assessed as up-to-date: 12 January 2015.

\begin{tabular}{|c|c|c|}
\hline Date & Event & Description \\
\hline 23 September 2015 & New search has been performed & $\begin{array}{l}\text { We updated the search to January } 2015 \text {. We included } \\
11 \text { new trials. These tested } 10 \text { new comparisons. Risk } \\
\text { of bias assessment replaced the previous assessment } \\
\text { of methodological quality. We assessed the quality of } \\
\text { evidence using GRADE and compiled the evidence in } \\
\text { summary tables }\end{array}$ \\
\hline
\end{tabular}

23 September 2015 New citation required and conclusions have changed
Although the conclusions reflecting the fundamental insufficiency of the evidence are unchanged, we have made some secondary revisions to the implications for practice, in terms of basic standards of care, and research

There has been a change to the byline.

\section{HIS T O R Y}

Protocol first published: Issue 4, 2001

Review first published: Issue 2, 2002

\begin{tabular}{l|l|l}
\hline Date & Event & Description \\
\hline 25 July 2008 & Amended & Converted to new review format. \\
\hline 9 May 2006 & New citation required and conclusions have changed & $\begin{array}{l}\text { In this substantive update (Issue 3, 2006) the search for } \\
\text { trials was updated to December 2005. We identified six } \\
\text { new studies and one full report of a trial formerly listed } \\
\text { as an ongoing study. Of these, we included three studies, }\end{array}$
\end{tabular}




\begin{tabular}{|c|c|c|}
\hline & & $\begin{array}{l}\text { excluded three studies and placed one study into 'Stud- } \\
\text { ies awaiting assessment'. We further excluded one study } \\
\text { previously awaiting assessment. There were several for- } \\
\text { mat changes made to comply with the Cochrane Style } \\
\text { Guide (October 2005). Graphical presentations of the } \\
\text { results were modified with totals removed in all cases. } \\
\text { There were no substantive changes made to the conclu- } \\
\text { sions. } \\
\text { For details of previous updates, please see 'Notes'. }\end{array}$ \\
\hline 25 July 2004 & Amended & $\begin{array}{l}\text { In the third, a minor update published in Issue 4, 2004, } \\
\text { all changes resulted from copy-editing. There were no } \\
\text { changes made to the conclusions }\end{array}$ \\
\hline 23 February 2004 & New search has been performed & $\begin{array}{l}\text { In the second, a minor update published in Issue } 3 \text {, } \\
2004 \text {, the search for trials was extended to January } 2004 \text {. } \\
\text { We identified no new studies nor publications of studies } \\
\text { listed as ongoing or pending. There were no changes } \\
\text { made to the conclusions }\end{array}$ \\
\hline 22 March 2003 & New search has been performed & $\begin{array}{l}\text { In the first, a minor update published in Issue } 2,2003 \text {, } \\
\text { the search for trials was extended to January } 2003 \text {. We } \\
\text { identified five new studies, three of which were ongoing, } \\
\text { one of which was excluded and one of which was placed } \\
\text { in studies awaiting assessment. There were no changes } \\
\text { made to the conclusions }\end{array}$ \\
\hline
\end{tabular}

\section{CONTRIBUTIONSOFAUTHORS}

For this update, JE performed the database searches and removed duplicates; HH performed most of the other searches. HH independently screened and selected studies, with screening and study selection by either JE or TH (see Acknowledgements) for the main databases including trial registers. Data extraction and risk of bias assessment were conducted by both authors. HH contacted trialists for further information, compiled the first draft and all subsequent revisions in RevMan. JE critically reviewed and checked all review drafts. $\mathrm{HH}$ is the guarantor of the review.

Past contributions of authors for the review protocol and previous versions of the review are available in this section of the previous version of the review (Handoll 2006). 


\section{DECLARATIONSOF INTEREST}

None known.

\section{SOURCES OF SUPPORT}

\section{Internal sources}

- University of Teesside, Middlesbrough, UK.

- Glasgow Caledonian University, Glasgow, UK.

- University of Manchester, Manchester, UK.

\section{External sources}

- National Institute for Health Research, UK.

This project was supported by the National Institute for Health Research (NIHR), via Cochrane Incentive Award funding to the Cochrane Bone, Joint and Muscle Trauma Group. The views and opinions expressed therein are those of the authors and do not necessarily reflect those of the Systematic Reviews Programme, NIHR, National Health Service (NHS) or the Department of Health

\section{DIFFERENCES BETWEEN PROTOCOLANDREVIEW}

Most of the changes to methods in the 2015 update reflect the uptake of new methodology and reporting as described in the Cochrane Handbook for Systematic Reviews of Interventions (Higgins 2011a). These include risk of bias assessment, more explicit reporting of data analysis and collection and assessment of the quality of the evidence using GRADE. Although not implemented in full, the primary outcome measures were identified and considered in the interpretation of trial results.

For consistency, we used the description of complex regional pain syndrome type 1 (CRPS-1) instead of reflex sympathetic dystrophy, which was the term commonly used in older studies.

\section{INDEX TERMS}

\section{Medical Subject Headings (MeSH)}

Fractures, Bone [rehabilitation]; Physical Therapy Modalities; Radius Fractures [* rehabilitation]; Randomized Controlled Trials as Topic; Wrist Injuries [* rehabilitation]

\section{MeSH check words}

Adult; Aged; Female; Humans; Male 